\title{
Designing game-based eHealth applications
}
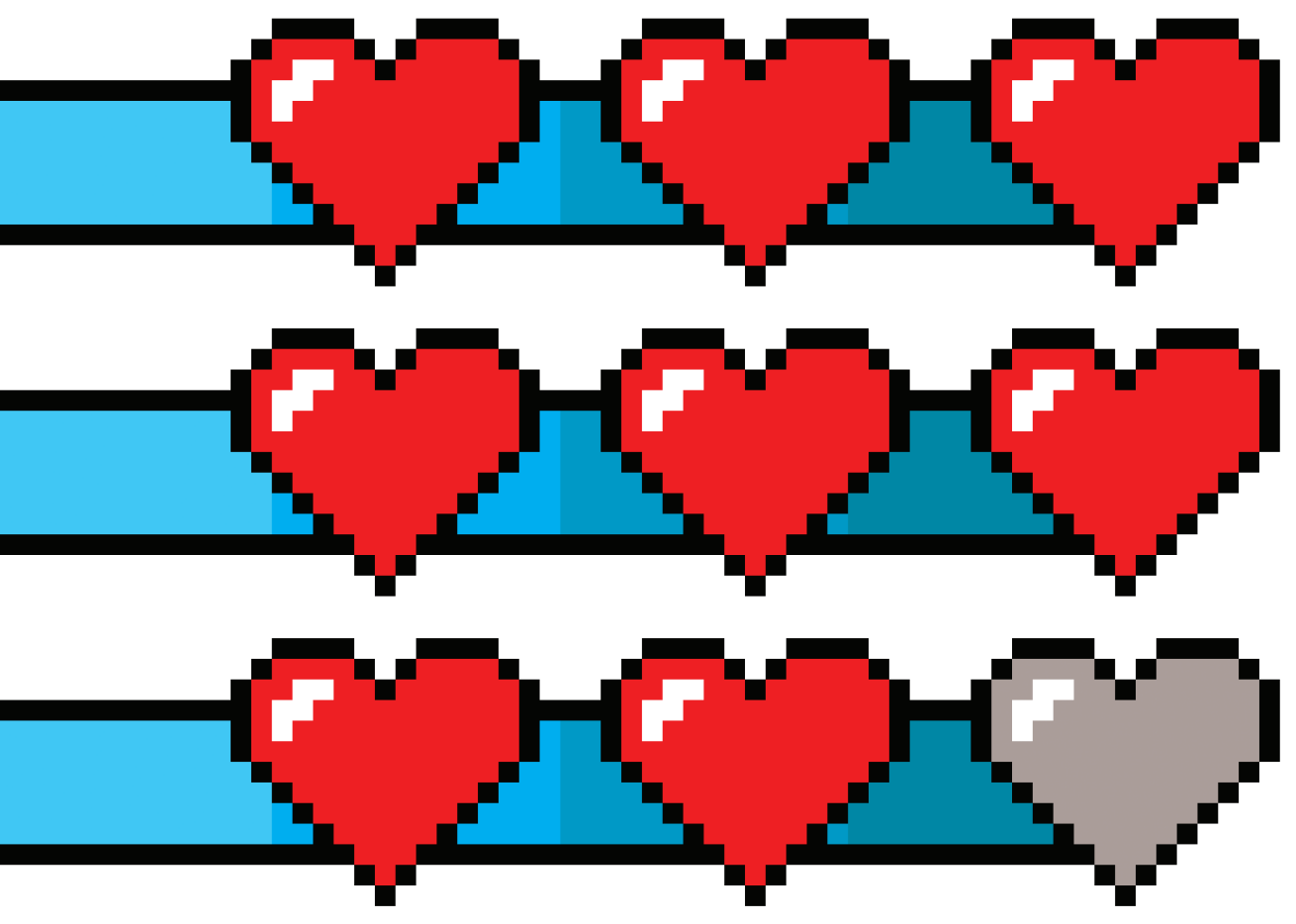

Strategies for sustainable engagement of older adults

Frederiek de Vette 



\title{
DESIGNING GAME-BASED EHEALTH APPLICATIONS
}

\author{
STRATEGIES FOR SUSTAINABLE \\ ENGAGEMENT OF OLDER ADULTS
}

Frederiek de Vette 
Correspondence:

Frederiek de Vette

frederiekdevette@gmail.com

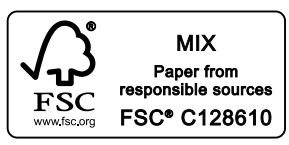

Chair Biomedical Signals and Systems

UNIVERSITY OF TWENTE.

\begin{tabular}{l|ll} 
UNIVERSITY & DIGITAL SOCIETY & $\begin{array}{l}\text { Digital Society Institute } \\
\text { DSI Ph.D. Thesis serie No. 19-0 1 } 1\end{array}$ \\
OF TWENTE. & INSTITUTE & P.O. Box 217,7500 AE Enschede, the Netherlands \\
UNIVERSITY & TECHMED & Technical Medical Centre \\
OF TWENTE. & CENTRE & P.O. Box 217,7500 AE Enschede, the Netherlands
\end{tabular}

ISBN: 978-90-365-4799-4

DOI: $10.3990 / 1.9789036547994$

ISSN: ISSN 2589-7721

Printed by: Ipskamp printing, Enschede

Cover and design: Frederiek de Vette

(C) Frederiek de Vette, Enschede, the Netherlands, 2019

All rights reserved. No parts of this thesis may be reproduced, stored in a retrieval system or transmitted in any form or by any means without permission of the author.

Alle rechten voorbehouden. Niets uit deze vitgave mag worden vermenigvuldigd, in enige vorm of op enige wijze, zonder voorafgaande schriftelijke toestemming van de auteur. 


\section{DESIGNING GAME-BASED EHEALTH APPLICATIONS}

\section{STRATEGIES FOR SUSTAINABLE ENGAGEMENT OF OLDER ADULTS}

\section{DISSERTATION}

to obtain

the degree of doctor at the University of Twente, on the authority of the rector magnificus, prof.dr. T.T.M. Palstra, on account of the decision of the Doctorate Board, to be publicly defended

on Wednesday the 1Oth of July 2019 at 12.45 hours

by

Anna Frederiek Alberdien Noorman - de Vette born on the 13th of April 1989

in Alkmaar, the Netherlands 
This dissertation has been approved by:

Supervisors Prof. dr. M.M.R. Vollenbroek - Hutten (promotor) Prof. dr. ir. H.J. Hermens (co-promotor)

Co-supervisor Dr. ir. M. Tabak 
Graduation Committee

Chairman/secretary Prof. dr. J.N. Kok University of Twente, EEMCS

Supervisors Prof. dr. M.M.R. Vollenbroek University of Twente, EEMCS

Prof. dr. ir. H.J. Hermens University of Twente, EEMCS

Co-supervisor

Dr. ir. M. Tabak

University of Twente, EEMCS

Members

Prof. dr. ir. R.M. Verdaasdonk University of Twente, TNW

Prof. dr. G.J. Westerhof University of Twente, BMS

Prof. ir. J. van Erp Delft University of Technology

Prof. dr. M.A. Neerincx Delft University of Technology

Prof. dr. M.C. Kaptein PDEng Tilburg University 



\section{TABLE OF CONTENTS}

8 Chapter 1| General introduction

16 Chapter 2| Engaging elderly people in telemedicine through gamification

34 Chapter 3| Exploring personality and game preferences in the younger and older population: a pilot study

46 Game preferences and personality of older adult users

50 Chapter 4| Mapping game preferences of older adults: a field study towards tailored gamified applications

68 How to design game-based healthcare applications for children? A study on children's game preferences

80 Chapter 5| The 6D Framework: an evidence-based tool for designers of game-based applications

100 Chapter 6| A gamified physical activity coaching application for older adults: design approach and user experience in daily life

122 Chapter 7| Game-based design for eHealth in practice

154 Chapter 8| General discussion

166 \& 


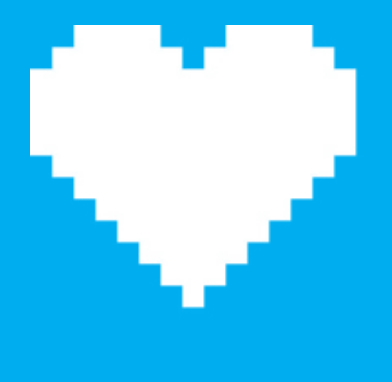

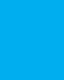


Play is an inherent human characteristic. According to some, play is even the foundation of all culture (Huizinga, 1938 [1]). By using the strong attractive force that games and play have on people, activities that we do not enjoy doing may be made more fun and better endurable. Or, as the Sherman brothers sang through the voice of Mary Poppins in 1964:

In every job that must be done

There is an element of fun

You find the fun and snap!

The job's a game

A spoonful of sugar helps the medicine go down

Using games and play to engage people is not a novelty strategy. Some historical examples are board games for teaching army tactics, consumer loyalty programs, educational toys and even road signs to prevent speeding in traffic. For this practice, the oxymoron 'serious games' was first described by Abt in 1970 [2]:

"Games may be played seriously or casually. We are concerned with serious games in the sense that these games have an explicit and carefully thought-out educational purpose and are not intended to be played primarily for amusement. This does not mean that serious games are not, or should not be, entertaining."

Since the 1980s, the era in which video games became an increasingly important part of entertainment industry, researchers in the field of Human-Computer Interaction worked on the idea to learn from games and apply parts of games in contexts not directly related to entertainment. Vastly pre-dating today's technology and digital world, Malone [3] discusses the question why games, specifically video games, are so captivating and how these features can be used to make other user interfaces more interesting and enjoyable. He proposes that it is intrinsic motivation that drives this interest, created by challenge, fantasy and curiosity.

As technology further advanced, interfaces gained a more prominent part in our lives. We see game elements being added to websites and smartphone applications as well, starting in marketing or 'consumer engagement'. Applications such as Nike+ that allow you to monitor your progress, compete with friends and earn status badges, effectively spike the sales of Nike's sports goods.

A new term for this phenomenon is gamification. While the exact origins of the term are not clear, it first emerges in 2003 and sees widespread use since end of 2011 (fig. 1). At that time, the Oxford Dictionary registers the following definition: 
"The application of typical elements of game playing le.g. point scoring, competition with others, rules of playl to other areas of activity, typically as an online marketing technique to encourage engagement with a product or service."

In industry, gamification is booming and the value of the gamification market is expected to grow from 4.91 billion U.S. dollars in 2016 to nearly 12 billion in 2021 . This success does not remain unnoticed outside of industry. An example occurring in science is Foldit (fig. 2), an application created through collaboration between Game Science and Biochemistry departments of the University of Washington. This online program challenges people to fold structures of protein molecules into a particular shape. The new structures that were produced by 57.000 players contributed to a number of medical breakthroughs. A 15-year old problem in the search for an HIV vaccine was solved in 10 days, thereby outperforming computer simulations.

In an attempt to conceptual ise gamification, several definitions have been formulated since its wide-spread adoption. Soon, discussion commenced on these various definitions and many developers felt the need to define their own interpretation of gamification to shield themselves from any misconceptions. The most commonly accepted definition from academia by Deterding et al. [4] is as follows:

"Gamification is the use of game design elements in non-game contexts."

The positioning of gamification relative to serious games can be clarified if we imagine games as parts or as a whole. Whereas gamification implies a system that only incorporates elements from games, serious games are full games with an explicit purpose. The underlying aims of a serious game are intertwined with the gameplay, while gamification uses game elements without constituting the basic structure of the underlying application itself. It is important to notice that the boundaries between the two concepts are sometimes vague, in word as well as in practice, and many different crossover forms may occur.

How these game elements should be defined and interpreted, in order to analyse game and user or to apply them in gamification, is in turn subject to different ideas. A framework that enables analysis of how games are experienced by the user, an important example from the field of game research, is proposed in 2004 by Hunicke et al. [5]. Their MDA framework divides game elements into mechanics, the base components of a game, dynamics, the behaviour of the mechanics acting on the input of the player, and aesthetics, the emotional responses the game evokes in the player. 


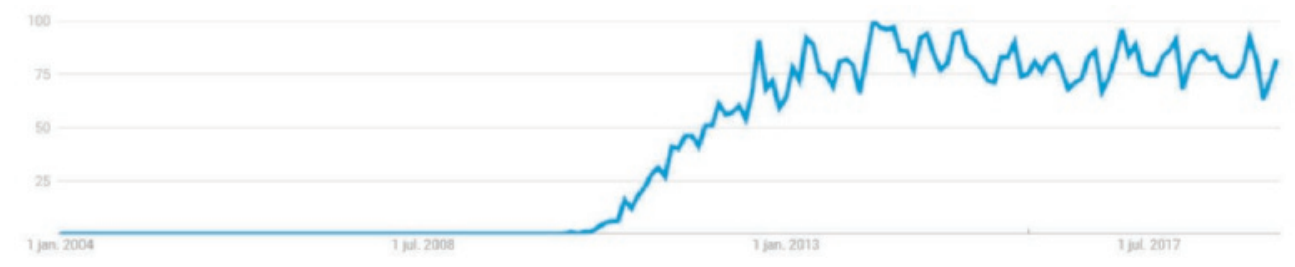

Figure 1 - Interest in gamification over time according to Google trends

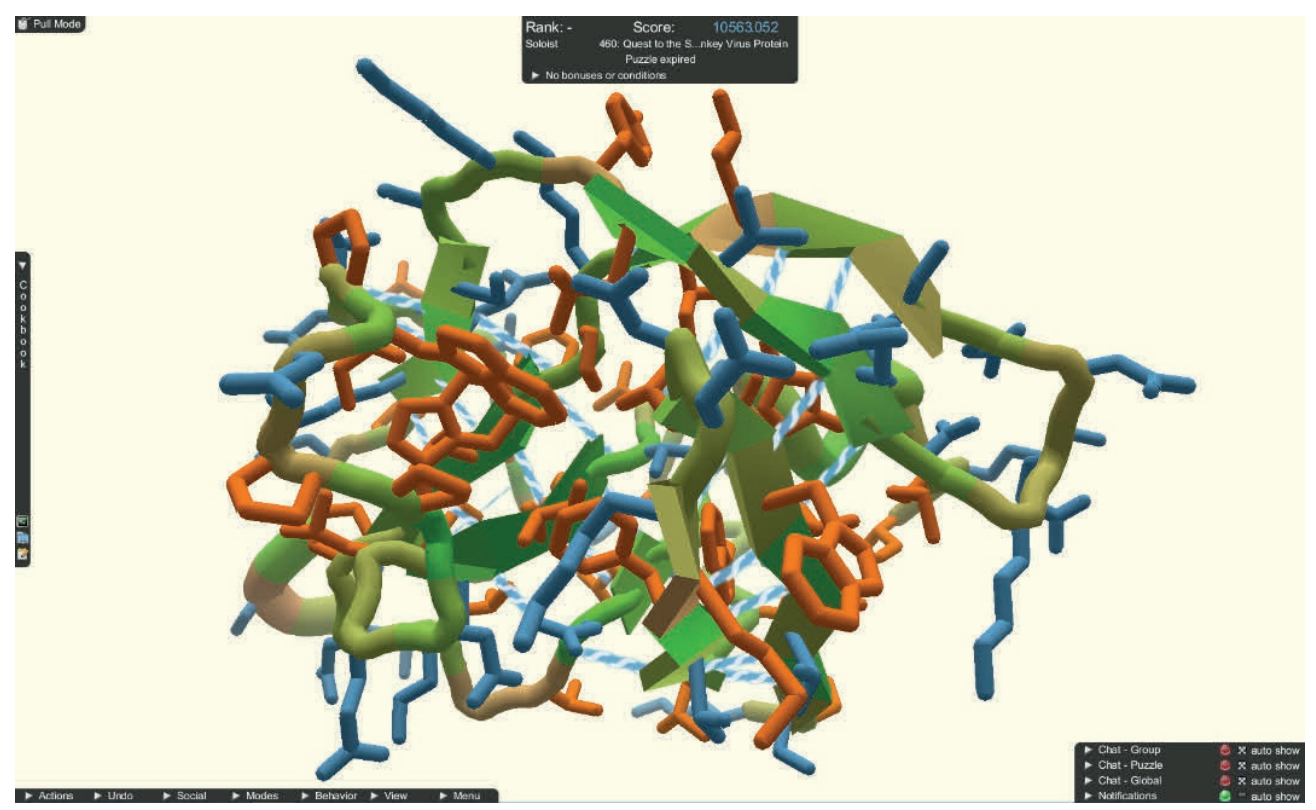

Figure 2 - Foldit, with user Madde's top scoring solution to the Mason pfizer monkey virus

In 2012, Werbach adds 'and game design techniques' to the Deterding definition and presents a simplified form of the MDA framework in which game elements are placed in a pyramid-shaped model [6]. The game elements consist of components, mechanics and dynamics, which are easily translated into design for an application. He presents an extremely popular MOOC (massive open online course) through which his 'ready to use' method to gamify any application gains a lot of followers.

While very practical and easy to understand, applying gamification as such has an important downside. Instead of focusing on the core experience of games, from which motivating elements should be deduced, the concept relies more on conditioning of behaviour through external conditions, or extrinsic motivation. While conditioning through gamification may not necessarily be bad practice, it can lead 
to unwanted effects as described by Przybylski et al. [7]. It may, for example, cause a shift from formerly intrinsic motivation to extrinsic motivation that leads to the loss of the initial interest in an activity once rewards are no longer offered, an effect named overjustification (Lepper et al. [8]).

The promise of gamification is that it can create an activity that is so much fun people would automatically be willing to work on underlying goals. A field that would benefit from a strategy like gamification has goals that very often require performing tedious, difficult, boring or strenuous tasks. A field in which motivation to perform these tasks is essential for positive outcomes. One such field is healthcare.

For the first time in history the number of elderly people, aged 65 and up, is larger than the number of children younger than five [9]. This phenomenon, called demographic ageing, occurs globally and creates a high old-age dependency ratio. The median age in Europe is already the highest in the world and it is predicted that $25 \%$ of the population is older than 65 in 2050, growing to almost $30 \%$ in 2080 $[10,11]$. This implies that there are 2,5 times more elderly people than young children. To illustrate this, the exact opposite was the situation in 1950 [12]. The demand for medical care increases fast because of demographic ageing, and an urgent shortage of care professionals strikes the Netherlands as well. The number of vacancies in healthcare currently hits 30.000 while over half of the population suffers from chronic illness. Of all people aged 65 and up in the Netherlands, 75\% is in need of chronic care [13].

Information technology can be used to help alleviate the increasing demand for care: eHealth. The umbrella term eHealth canopies areas of research such as health informatics, mHealth, telemedicine and telehealth, home automation (domotics) and personalised medicine. Eysenbach (2001) defines the concept of eHealth as follows [15]:

"e-Health is an emerging field in the intersection of medical informatics, public health and business, referring to health services and information delivered or enhanced through the Internet and related technologies. In a broader sense, the term characterizes not only a technical development, but also a state-of-mind, a way of thinking, an attitude, and a commitment for networked, global thinking, to improve health care locally, regionally, and worldwide by using information and communication technology."

The healthcare system can benefit from eHealth in numerous ways. eHealth can empower healthcare providers by streamlining care processes and efficiently manage and share health data as well as enable remote monitoring and advising for patients [16]. The quality of healthcare can be supported by making use of big data solutions, integrating relevant treatment and outcome data [17]. In addition, 
eHealth can empower the patient by improving accessibility to healthcare and optimising self-management [18-20]. This can for example support preventive care through managing modifiable health risk behaviours and avoiding patients developing chronic conditions [21,22].

This promising technology however faces an important challenge. As is the case for treatment without use of technology, the use of eHealth applications as intended by the caregiver and needed to optimally benefit from the treatment offered leaves much to be desired $[23,28]$. Therefore, strategies are needed that can make eHealth applications more engaging in order to delay this drop in so-called adherence as long as possible.

A target group that can particularly benefit from this technology is the older adult. For them, eHealth can contribute to maintaining autonomy and independence. For example, eHealth can support adopting a more active lifestyle that results in lower institutionalisation and an improved quality of life [29]. However, knowledge on how to effectively engage the older adult in the use of eHealth is not abundant. Designing for this target group is further challenged by its heterogeneity in terms of computer literacy, openness to the use of technology and age-related limitations.

In this thesis we aim to combine the potential of gamification to engage people in productive activities outside of entertainment with the need for strategies to improve engagement to eHealth applications. Specifically, we focus on addressing the older population. The objective of this thesis therefore is:

\section{To research how to design game-based applications that engage elderly users in eHealth.}

This thesis is based on two lead incentives. In Chapters 2-5, research is focused on a method to capture the elements of games that intrinsically motivate people to play, to investigate the elements that are motivating for elderly users, and how these can be translated into engaging design of gamified applications. Chapters 6-7 cover the development and evaluation of two gamified eHealth applications for the older adult, in which the results of the first section are applied.

To study the status quo, we started by gaining insight in existing theoretical frameworks for gamification and user classifications to engage elderly users through a literature study, as described in Chapter 2. As we found that gamification for the older adult was scarce, it remained uncertain which motivational concepts from games could be utilised to develop suitable content for this target group. Therefore, as described in Chapter 3, the results from this previous chapter lead to the exploration of the conceptual basis for a novel framework to assess and map preferences for game content for specific target groups, regardless of age, by 
means of an online pilot study. Described in Chapter 4-5, studies aimed at refining the framework to be more accurate in expressing game content and on designing a survey most suitable for specific target groups. Chapter 4 describes a field study in which the preferences of older adults after playing several tablet games in home setting were assessed and mapped. Results included recommendations for game design for older adults. Consecutively, the framework was used to assess game preferences of children aged 6-12, leading to design recommendations for this target group. Concluding the first section, Chapter 5 presents the finalisation of the now called 6D framework by means of an expert and user evaluation study. This resulted in a practical tool for developers of gamified applications that enables assessment of any target group's preferences.

Chapter 6 describes the design approach of MAGGY, a game-based mobile coaching application that aims to stimulate physical activity of older adults in daily life. In this study, resulting recommendations for game design from Section A were applied and evaluated. In Chapter 7, cumulative knowledge from all previous studies was used in the second project, PERSSILAA, a service for screening and prevention of frailty by screening, monitoring and training. Here, we designed, developed and evaluated the game 'Stranded!'. This study presents the design trajectory of an entire game-based eHealth application for the older adult from front to end.

Finally, in Chapter 8 the findings of this research are discussed in a broader perspective. In this chapter we reflect on our findings resulting from our main objective and consider their practical and future implications.

Over the course of this research our developments would be positioned in the area between serious games and gamification, and could not be described well anymore by the original definition of gamification (nor of serious games). The broader term game-based design was therefore adopted, covering more forms of games applied in initiatives other than entertainment. 


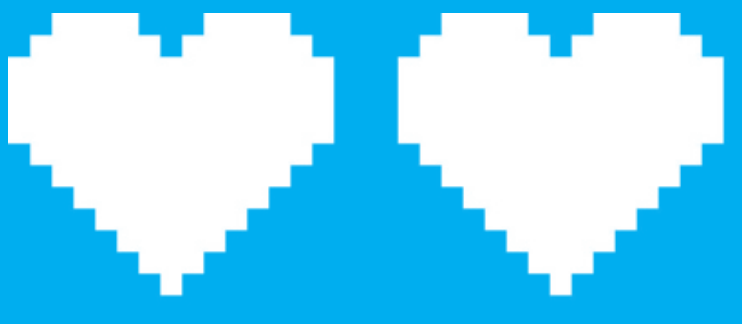

$$
\text { . }
$$

.

.

(

(

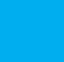

( 


\section{Abstract}

\section{Background}

Telemedicine can alleviate the increasing demand for elderly care caused by the rapidly aging population. However, adherence to technology in telemedicine interventions is low and decreases over time. Therefore, there is a need for methods to increase adherence, specifically of the elderly user. A strategy that has recently emerged to address this problem is gamification. It is the application of game elements to non-game fields, to motivate and increase user activity and retention.

\section{Objective}

This research aims to 1) provide an overview of existing theoretical frameworks for gamification and explore methods that specifically target the elderly user, and 2) explore user classification theories for tailoring game content to the elderly user. This knowledge will provide a foundation for creating a new framework for applying gamification in telemedicine applications to effectively engage the elderly user by increasing and maintaining adherence.

\section{Methods}

We performed a broad internet search, including scientific and non-scientific search engines, and included information that described either of the following subjects: the conceptualisation of gamification, methods to engage elderly users through gamification, user classification theories for tailored game content.

\section{Results}

Our search showed two main approaches concerning frameworks for gamification: from business practices, which mostly aim for more revenue, emerges an applied approach, and from academia frameworks are developed incorporating theories on motivation while often aiming for lasting engagement. The search provided limited information regarding the application of gamification to engage elderly users, and a significant gap in knowledge on the effectiveness of a 'gamified' application in practice. Several approaches for classifying users in general were found, based on archetypes and reasons to play, and present them along with their corresponding taxonomies. The overview we created indicates great connectivity between these taxonomies.

\section{Conclusion}

Gamification frameworks have been developed from different backgrounds business and academia - but rarely target the elderly user. The effectiveness of user classifications for tailored game content in this context is not yet known. As a next step, we propose the development of a framework based on the hypothesised existence of a relation between preference for game content and personality. 


\section{1 | Introduction}

It is expected that $25 \%$ of the European population will be older than 65 years in 2050 because of global population aging [1]. Current socioeconomic structures cannot provide enough work force and capital to meet the needs of this rapidly growing elderly population [2]. Telemedicine refers to health services that enable patients to receive treatment in their daily living environment, whereby distance is bridged by information communication technology (ICT) and at least one healthcare professional is involved, alleviating the increasing demand for elderly care by extending the time of autonomy and independence [3]. Though telemedicine technology seems promising, practical implementation still leaves much to be desired. Several studies have shown that adherence to telemedicine interventions, such as therapy supporting a healthy lifestyle, is low [4] and decreases over time [5], even though these studies showed a significant effect on health outcomes [6]. Clearly, there is a need for strategies that motivate elderly people to use, and keep using, the technologies offered.

Gamification, the application of game elements to nongame fields, may be such a strategy [7]. There is a rapid growth in the number of initiatives that use gamification, illustrating a variety of approaches developed from various viewpoints, including education, behaviour change, physical health, and mental health. However, a lack of a refined conceptualisation of this strategy exists in these disciplines, and gamification, for elderly people in particular, remains an even further underexplored area. In general, it is not yet known which one of these approaches is the best for the durable engagement necessary for better adherence.

Choice and personalisation of content [8], or tailoring, is known to be beneficial for intrinsic motivation [9], which in turn increases long-term engagement needed for adherence. To provide this tailored content, insight is needed into how users should (or want) to be addressed through gamification and how these needs can be classified is required. To our knowledge, information on the practical implementation of existing classifications is not yet available. We believe that once an overview of existing frameworks for gamification and user classification is established, a gamification strategy that is effective in realising long-term engagement for the elderly user can be developed.

For this purpose, the aim of the paper is to (1) provide an understanding of the theoretical background of gamification, including existing frameworks for developing gamification both in general and specifically for the elderly, and (2) explore existing user classification theories that may serve for the tailoring of game content to the target user. Because of the newness of this field of research, we opt for a broad view on activities in gamification which occur not only within but also outside of 
Table 1 - Keywords first search.

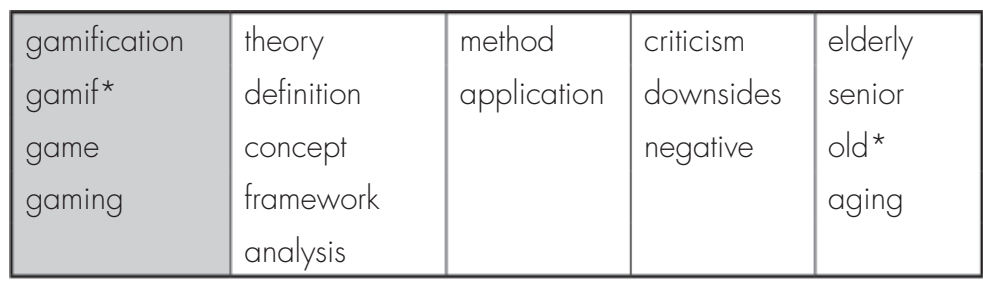

Table 2 - Keywords second search.

\begin{tabular}{|l|l|}
\hline user & type \\
player & taxonomy \\
gamer & classification \\
& model \\
& theory \\
& style \\
\hline
\end{tabular}

scientific research. In future research we will work towards a user classification of the elderly that can be used to develop evidence-based gamification strategies and tangible design guidelines for gamification in healthcare.

\section{2| Methods}

In a succession of three Internet searches, a broad approach to the subject of gamification was taken in order to gain insight in the many developments in gamification that occur both inside and outside of the scientific world. We performed a search in the scientific search engines PubMed, Scopus, and Google Scholar and in diverse non-scientific sources: from game designer blogs and conference videos to MOOCs (massive open online courses) and YouTube videos. In this paper, gamification is defined as the use of elements from games in nongame contexts to improve user experience and engagement without making that system a full game as is the case with serious games including exergames /combination of exercise and gaming) $[10,11]$.

First, we have researched the conceptualisation of gamification from a theoretical perspective (Table 1). Keywords used in combination with gamification were used, including derivatives of these words: "theory," "definition," "concept," "framework," and "analysis." Also, keywords (and derivatives of these) implying practical use were used: "method," "application," and "gamify" (singular). Then, a search 
for gamification combined with "criticism," "downsides", and "negative" was performed. Secondly, we investigated the use of gamification in applications for the elderly (Table 1), entering the following combinations of keywords: "gamification," "gamif*," "game," and "gaming" with "elder*," "elderly," "senior," "old*," and "aging." Finally, through the same search method, we have researched user classifications that categorise users by their motivation or stimulant to play in order to gain insight into the user and further determine how to tailor content to the user (Table 2). Keywords used were "[user, player, gamer]" combined with "[type, taxonomy, classification, model, style]."

Included in the results were articles and other works that present a theoretical basis for the development of gamification, defined as the presence of a framework that is either theoretical and/or based on established scientific foundations or proven effective through evaluation in practice. Therefore, beyond the scope of our paper are numerous works on gamified applications with a black box design.

\section{3| Results}

\subsection{Gamification frameworks}

This section demonstrates the current state of gamification, starting with the concept of gamification in a broader sense and then focusing on gamification for elderly people. We provide an overview of existing frameworks for gamification along with their contexts and backgrounds. With this, we aim to define the status quo in research and provide a deeper understanding of the concept and its use and misuse.

\subsubsection{The conceptualisation of gamification}

Gamification has gained popularity in diverse fields such as (interactive) marketing and scientific applications, generating different definitions of gamification. Currently there is no consensus about a definition, mainly due to the underlying perception of what the game elements are exactly in terms of level of abstraction and whether the gamified application is game-like or not. Gamification is often roughly defined as the use of elements from games in nongame contexts; a more refined definition regards gamification as the identification of that which makes games captivating and engaging followed by the transfer of this knowledge to nongame contexts, increasing user enjoyment $[12,13]$. While some see gamification as a way to act upon psychological principles as certain game techniques do [14], others define gamification as applying gameful interaction or design with a specific intention without creating a full-fledged game [10] or as the process of improving a service with gameful experiences that support the value creation of the user [15]. In the middle of these definitions, we see gamification as the use of game elements that create a game-like experience in a nongame context without creating a full game. 
We found two approaches towards the conceptualisation of gamification. One emerges from business practices, such as marketing, customer loyalty, and employee engagement; the other from academia and not sales driven, often specifically aiming to incorporate theories on motivation, engagement, and behaviour change. Table 3 illustrates this division of the found articles by author, grouped according to their focus.

In business-oriented, or corporate, gamification, the number of successful initiatives, in terms of increased user engagement or revenue, that use gamification has been rapidly increasing in the past few years [16]. It is estimated that the market spend on gamification solutions will grow exponentially until 2016 , and at that time $40 \%$ of the world's top market value companies will be using gamification $[17,18]$. In gamification for the marketing of consumer products, a well-known success story is that of Nike+ by Nike. This gamified running log app, currently used by five million players to track their daily exercise goals, caused revenues in the running category to increase by $30 \%$ in 2011 alone [19]. An example of successful enterprise gamification is that of software company SAP. After SAP launched a new, gamified version of their online employee and customer community platform, employee usage increased by $400 \%$ and community feedback by $96 \%$ [20]. Gamification appears to be more than a fad, illustrated by the existence and ongoing success of companies such as Badgeville $[21,22]$, which provides a platform for gamification of enterprise applications and serves major companies such as Samsung, Deloitte, and Dell [23].

There are several authors within this business orientation, such as Cunningham and Zichermann [12], who provide guidelines for gamification by listing game elements and mechanics such as feedback, achievement, social engagement loops, reinforcement, and status, including practical examples. Werbach and Hunter [14] simplify gamification and consider it a tool for business strategy. Their method offers practical guidelines on how to dissect existing games and using them to gamify other applications. Although this approach lacks intricate game mechanics, gamification is used as a comprehensible tool, presenting game elements as a set of building blocks that, used together, can provide the gamified application.

On the other hand, the way gamification is applied in business context receives a lot of criticism as analysts estimate that the bigger part of current gamified applications will not meet their business objectives, mainly due to poor design [24]. Game designers criticise the Cunningham and Zichermann method, stating that the mechanics presented do not contribute to a gameful experience $[25,26]$. Robertson states that gamification turns into "pointsification" when game elements are simply stripped from games and placed in another application [25]. With this, structural components of games are perceived and used elsewhere to function as core mechanics, ignoring the fact that these mechanics should be the inner 
Table 3 - Frameworks for gamification in business and academia.

\begin{tabular}{ll} 
Business & Academia \\
\hline Cunningham and Zichermann (2011) & Aparicio et al. (2012) \\
Werbach and Hunter (2012) & Nicholson (2012) \\
Duggan (Badgeville, 2012) & Sakamoto et al. (2012)
\end{tabular}

workings of games. Bogost criticises this practice using the term "exploitationware" in an article [26] and blog entry titled "Gamification is Bullshit" [27] and states that gamification disassociates the practice from games created for the sole purpose of making an easy profit. A design may be poor as well when it extensively uses external conditions or reinforcements, as known from operant conditioning [28]. These reinforcements often function as main mechanisms to manipulate behaviour and usually present in the form of point and reward systems. A shift from intrinsic to extrinsic motivation can occur through offering external awards, known as the overjustification effect [29], which may lead to an early loss of interest of the user. The initial interest in the (gamified) activity may also disappear once the rewards are no longer, or insufficiently, offered [30], an effect called the hedonic treadmill [31 ]. From this we observe that the development of a good game design concept is often disappearing into the background in corporate gamification initiatives, while it is as essential for creating an engaging experience as it is for traditional games.

Scientific research from within academia, the second approach we distinguish, includes few frameworks on the theoretical foundations of gamification. Aparicio et al [32] developed a framework focusing on intrinsic motivation by incorporating concepts from self-determination theory [33]. According to this theory, intrinsic motivation can increase by satisfying the three psychological factors: competence, autonomy and relatedness. The framework procedure tells us to (1) identify the main objective, (2) identify which intrinsically motivating factors should be included, (3) determine which game mechanics should be used according to these factors, and (4) evaluate the framework in its final application. Nicholson [34] presents a complex framework for meaningful gamification, integrating user-centred design [35] in combination with self-determination, situated motivational affordance [36], situational relevance [37], and universal design for learning [38]. From these core theories, Nicholson suggests how to provide more intrinsically motivating gamification leading to meaningful engagement. Self-determination can be found along with the transtheoretical model of behaviour change [39] in the framework of Sakamoto et al [40], describing a value-based framework. The authors present five core values (informative, empathetic, persuasive, economic, and ideological value) that, when used with other game mechanics, can be used to create attractive and intrinsically motivating gamification services. 
Table 4 - The contrast between business and academic frameworks.

\begin{tabular}{|c|c|}
\hline Business & Academia \\
\hline Applied & Conceptual \\
\hline Simplicity & Complexity \\
\hline Practical guidelines & Methods inexplicit \\
\hline Proven worthy in practice & $\begin{array}{l}\text { Earlier stage of development, } \\
\text { less empirical support }\end{array}$ \\
\hline Lacking depth, oversimplified & Solid scientific foundation \\
\hline Short-term engagement suffices & Aiming for durable motivation \\
\hline Immensely popular & Mostly unknown \\
\hline
\end{tabular}

Several differences between the frameworks from business and academia (Table 4) can be observed. The business frameworks are very concrete; they are simple, provide practical guidelines, and, most importantly, have proven their success in this context. In academia, gamification has not yet reached this state of maturity. The frameworks found on both sides are contradictory: those from academia are conceptual and complex and provide methods that are much more difficult to apply. Therefore, among these are no empirically supported frameworks showing their effectiveness in practice. The frameworks from business are simplified, therefore lacking depth, which may suffice for marketing purposes but possibly not for longterm goals needed for telemedicine applications.

\subsubsection{Gamification for elderly users}

While gamification is gaining popularity in telemedicine [41], limited information was found on appropriate designs for engaging elderly users. Our search for gamification frameworks did not return any information on how to address the elderly. We therefore present existing literature that describes explorations of designing gamification for the elderly (Table 5). Gerling et al indicate that gamification holds significant potential for elderly users, particularly in gamifying physical and cognitive therapy [7]. The authors state that the main challenge for developing such apps lies within the unfamiliarity of older adults with games, making it difficult to draw content from existing digital games. Link et al face a similar challenge after examining a set of game mechanics (points, status, and badges) and concluding that these have the desired impact on youth but not on older adults [42].

In contrast, Minge et al see gamification as an opportunity to decrease feelings of fear and frustration that elderly people have towards technology. However, the authors emphasise that success depends on careful design. For example, the study participants did not enjoy aspects of quantification and comparison [43], which are otherwise very common elements of games. 


\section{Table 5 - Overview of papers described.}

\begin{tabular}{ll} 
Source & Topic \\
\hline Usselsteijn et al. (2007) & Design opportunities for engaging games for elderly \\
Gerling et al. (201 1) & Potential of gamification for engaging (frail) elderly \\
Minge et al. (201 1) & Attitude of elderly towards gamification \\
Link et al. (2014) & Effect of game elements on motivation of elderly
\end{tabular}

Issselsteijn et al [44] also state that digital games hold significant positive potential for elderly users, including therapeutic value and social bonding. Elderly users are underrepresented as consumers of digital games because the games offered are not in line with their accessibility and usability demands or their interests and needs. Design requirements are needed to offer the elderly engaging content. According to Isselsteijn, however, no empirical data are available on the categorisation of elderly gamers that is necessary to do so, including how this would translate into game content.

\subsection{Classifying users: player taxonomies}

User classification holds a key role in the development of tailored game content, as it gives thorough insight into the preferences that individuals or subgroups within a target group may have [45]. However, there are limited valid methods to describe people regarding their gaming preferences [32], and none were found for the elderly user in particular [44]. In this section, we discuss several approaches for classifying users in general, broadly divided into archetypes and reasons to play. Archetypes, player types $[46,47]$ (Bartle, Marczewski), and gaming personality (types) [48] (Vandenberghe) describe the player characteristics while reasons to play, player motivation $[49,50]$ (Yee), and kinds of fun $[51,52]$ (LeBlanc, Lazzaro) take motivating elements as a starting point. In Figure 1, these various approaches are visualised in a diagram. At the end of this section, we summarise and compare these user taxonomies in a chart.

\subsubsection{Archetypes}

The earliest and most cited player taxonomy in a gaming context is the Bartle player type theory. It was developed for the first virtual multiuser environment, textbased dungeons (multiuser dungeons, or MUDs), by observing and analysing player patterns. Bartle proposes four player types (Figure 2) based on two primary interests in gameplay: between the emphasis on players or on the environment and between acting (to) and interacting (with). Achievers are interested in actions on the world and find mastery of the game and competition most compelling; explorers like to interact with the world and enjoy discovery. Socialisers are most interested in interacting with other players and enjoy the game for friendships and contacts, while killers are interested in acting on other players, demonstrating their superiority. 


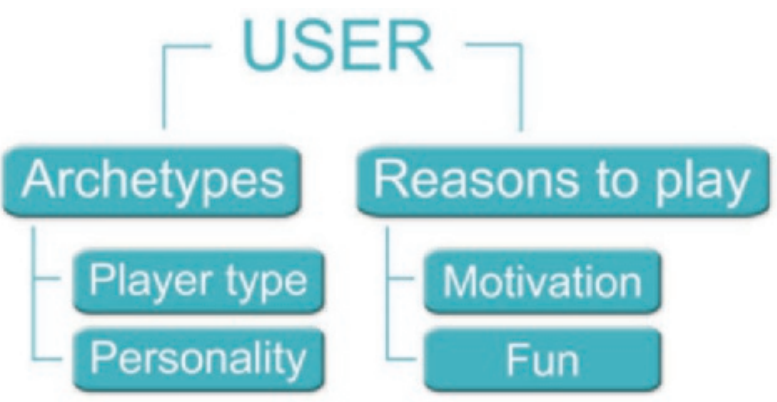

Figure I - Approaches to classifying the user

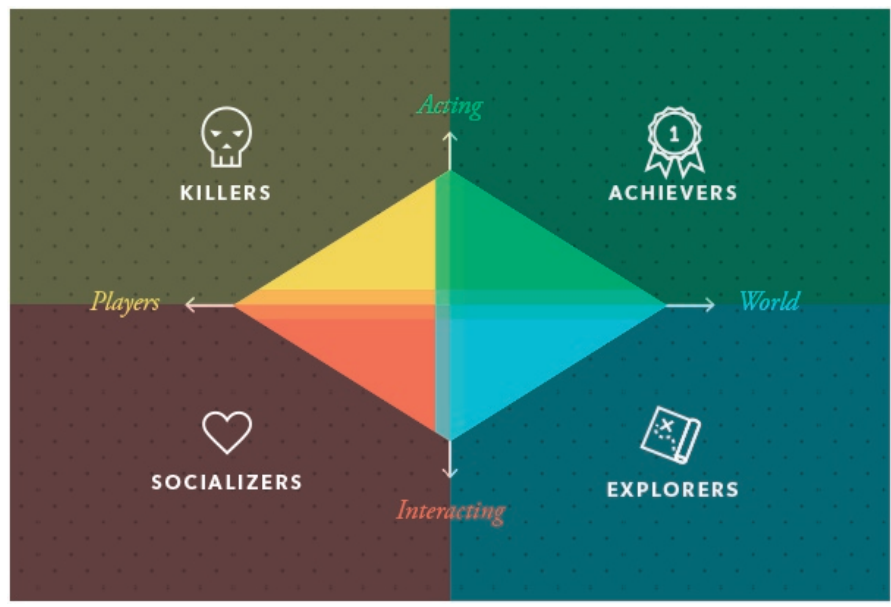

Figure 2 - Bartle's player type model[55]

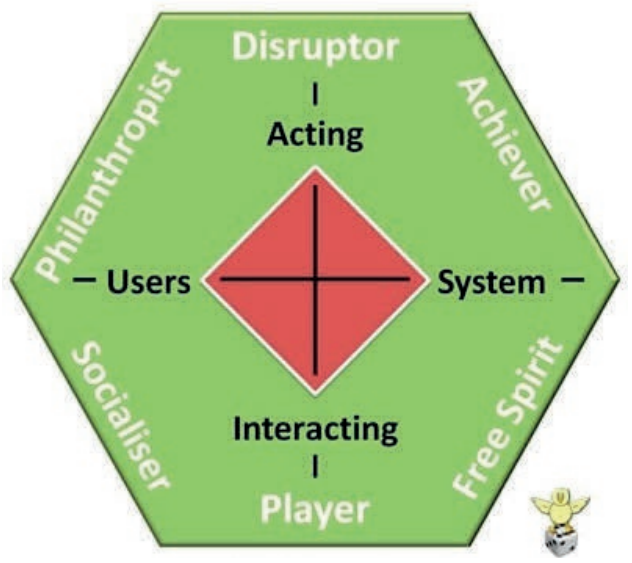

Figure 3 - Marczewski's player type model[54] 
According to Bartle, a good MUD contains the four player types in equilibrium [46]-not necessarily of equal number-and the player types were created to balance the design of these multiplayer games to accommodate for all player types' play style. The application of this model outside its context is something Bartle himself advises against [45], especially for use in gamification. Furthermore, this model has been criticised for lacking proper validation with empirical data and means to assess players to a type $[53,49]$ and for missing similarity between the virtual world of the MUD and the gamified application. Bartle suggests that the types are exclusive but in practice they can be overlapping or mixing [12].

Similarly, but in the context of enterprise gamification, Marczewski proposes a conceptual taxonomy choosing intrinsic motivations from different theories [54]: autonomy; purpose and mastery; change; and the extrinsic motivation, rewards. This results in six player types (Figure 3). The axes are equal to the Bartle model but replace player for user and world for system.

Another approach to create player archetypes is through personality. Personality traits have been extensively studied and researched since the 1880s [56] and, although thousands of traits can be found to describe personality [57], a statistical factor analysis demonstrated five main factors that many psychologists believe are sufficient $[58,59]$. The five factor model (FFM), or Big Five, is currently the most popular and has shown to be reputable, predictive (even normally distributed), reliable, cross-culturally tested, and universal [60-64].

In the context of games and gaming, several attempts on predicting the effectiveness of the application of FFM showed inconsistent results $[65,66]$. In one study, personality traits have been related to preference for game genres [67]. A low predictive capability was found, which may be caused by lack of evidence whether the FFM is a valid method to measure personality in a game or not $[68,69]$; however, direct correlations between the FFM and gaming were researched and described by Vandenberghe [48]. He states that personality is very accurately predictive of gaming preferences and that people play with the same motivations they have in real life or look to express a particular part of personality that is unsatisfied in real life. In his model, the five domains of play, a translation of the original FFM traits is made into aspects of gaming motivation (Table 6). Each player is ranked on a linear scale on each of the five domains, thereby creating a character description rather than a categorisation into a single player type. At the same time, the domains provide insight into the type of content that satisfies the player.

Two examples illustrate specific gaming elements derived from motivation facets. First, the imagination of the user correlates with a preference for either fantasy or realism: someone who scores high on imagination will tend to prefer games that take place in exotic worlds, while someone with a low score will prefer games that 
Table 6 - Five factor model traits and corresponding gaming motivation traits (deduced from Vandenberghe, 2012).

\begin{tabular}{|rcl|}
\multicolumn{1}{c}{ low score } & \multicolumn{1}{c}{ trait } & high score \\
\hline Cautious, predictable & Openness to Experience & Inventive, curious \\
Repeating, conventional & Novelty & Open, imaginative experiences \\
Careless, impulsive & Conscientiousness & Efficient, organised \\
Low effort and self-control & Challenge & High effort and self-control \\
Reserved, solitary & Extraversion & Energetic, outgoing \\
Relaxing, low social engagement & Stimulation & Exciting, high social engagement \\
Analytical, detached & Agreeableness & Friendly, compassionate \\
Competition, defeating & Harmony & Cooperation, helping \\
Confident, secure & Neuroticism & Nervous, sensitive \\
Cheerful, comforting & Threat & Gloom, horror, high tension \\
\hline
\end{tabular}

take place in a world much like ours. Second, scoring high on adventurousness correlates with a preference for exploration and a desire for encountering new things, much like the Bartle type explorer, while a low score indicates a preference for local play styles such as building or farming that do not involve leaving the boundary of the known [70].

\subsubsection{Reasons to play}

Yee proposes a taxonomy based on users' reasons to play and used a long-term, qualitative analysis and factor analytical approach to create a taxonomy based on player motivations in MMORPGs (massive multiplayer online role-play games). The model by Yee consists of ten subcomponents factored into three main components with which they are most correlated (Figure 4). Each subcomponent is linked to game elements from which players derive satisfaction. He finds that the killer must be omitted and merged into his component of achievement and the original explorer type must be divided into mechanics and discovery. The Yee model is similar to Bartle's but overcomes several of its weaknesses. For example, the components of Bartle types are not highly correlated, the types overlap and are not distinctive, and a practical way to assess users is lacking [71]. However, similar to the Bartle typology is its narrow focus on massive online gaming.

A taxonomy of game aesthetics, or what makes a game fun, can be found in the mechanics, dynamics, and aesthetics framework by LeBlanc [51]. Eight kinds of fun are defined: sensation, fantasy, narrative, challenge, fellowship, discovery, expression, and submission. These aesthetics are used to describe why certain players engage with certain games and more regard the game than categorise the player. Similarly, also focusing on fun as a reason to play, Lazzaro conducted 
a study to clarify how to address emotions in games without using a storyline by learning what (adult) players found were good gaming experiences [52]. The "four keys" to fun are:

Hard fun: players like challenge, strategy, problem solving, experiencing frustration Easy fun: players like intrigue and curiosity and enjoy immersion.

Altered states: players search for internal sensations such as excitement.

The people factor: players use games for social experiences.

\subsubsection{Overview of taxonomies}

Although the taxonomies presented above appear very different concerning the types of classes, many parallels can be found between the characteristics of each class. We present the results in an overview chart (Figure 5). The top row in gray shows the author of the model, and under each author the defined classes Itypes, motivations, facets, etc.) are shown. Arrows indicate a direct derivative of a model, as explained in the previous section; black lines indicate which classes show highly similar characteristics. The dotted line indicates that classes only have several characteristics in common. The colors indicate which classes belong to the same group. This overview shows that there is great connectivity between the models and highlights that the model of Vandenberghe covers all class properties of the other models (except for the player in the Marczewski model).

In the models of Marczewski and Yee, which both have Bartle as point of reference, we see a clear analogy between the achievers and socialisers, and also in the attributes of the free spirit (interacting with the system, autonomy), the explorer (interacting with the world), and immersion (discovery, exploration). Although Yee does not have a separate type for the killer or disruptor, provocation and domination are present in achievement. Linking to Lazzaro and LeBlanc, achievement is similar to the concept of hard fun and challenge, easy fun, Iwhich includes the motive of immersion) and discovery are similar to exploring, and the people factor and fellowship and expression relate to the social aspect. The model of Vandenberghe not only seems all-embracing, it adds a dimension to each personality trait. The killer can be linked to a very low score on harmony, the achiever to a high score on challenge, the explorer to a high score on novelty, the socialiser to a high score on stimulation. The trait threat is quite unique and only linked to submission. According to Vandenberghe, this trait may not be pointing out what keeps a player playing but what makes the player decide to stop playing.

None of the taxonomies presented target the elderly user specifically. Furthermore, we do not know of any methods regarding the mapping of this target group on the existing taxonomies, mainly because the gaming industry does not focus on this group as a consumer for video games. Moreover, the taxonomies are in most cases designed for use in a specific application, such as enterprise gamification or 


\begin{tabular}{|c|c|c|}
\hline Achievement & Social & Immersion \\
\hline Advancement & Socializing & Discovery \\
Progress, Power, & Casual Chat, Helping Others, \\
Accumulation, Status & Making Friends & $\begin{array}{c}\text { Exploration, Lore, } \\
\text { Finding Hidden Things }\end{array}$ \\
\hline Mechanics & Relationship & Role-Playing \\
Numbers, Optimization, & Personal, Self-Disclosure, & Story Line, Character History, \\
Templating, Analysis & Find and Give Support & Roles, Fantasy \\
\hline Competition & Teamwork & Customization \\
Challenging Others, & Collaboration, Groups, & Appearances, Accessories, \\
Provocation, Domination & Group Achievements & Style, Color Schemes \\
\hline & & Escapism \\
\hline & & Relax, Escape from RL, \\
\end{tabular}

Figure 4 - The components and subcomponents (Yee's model Motivations of Play in MMORPGs)[49]

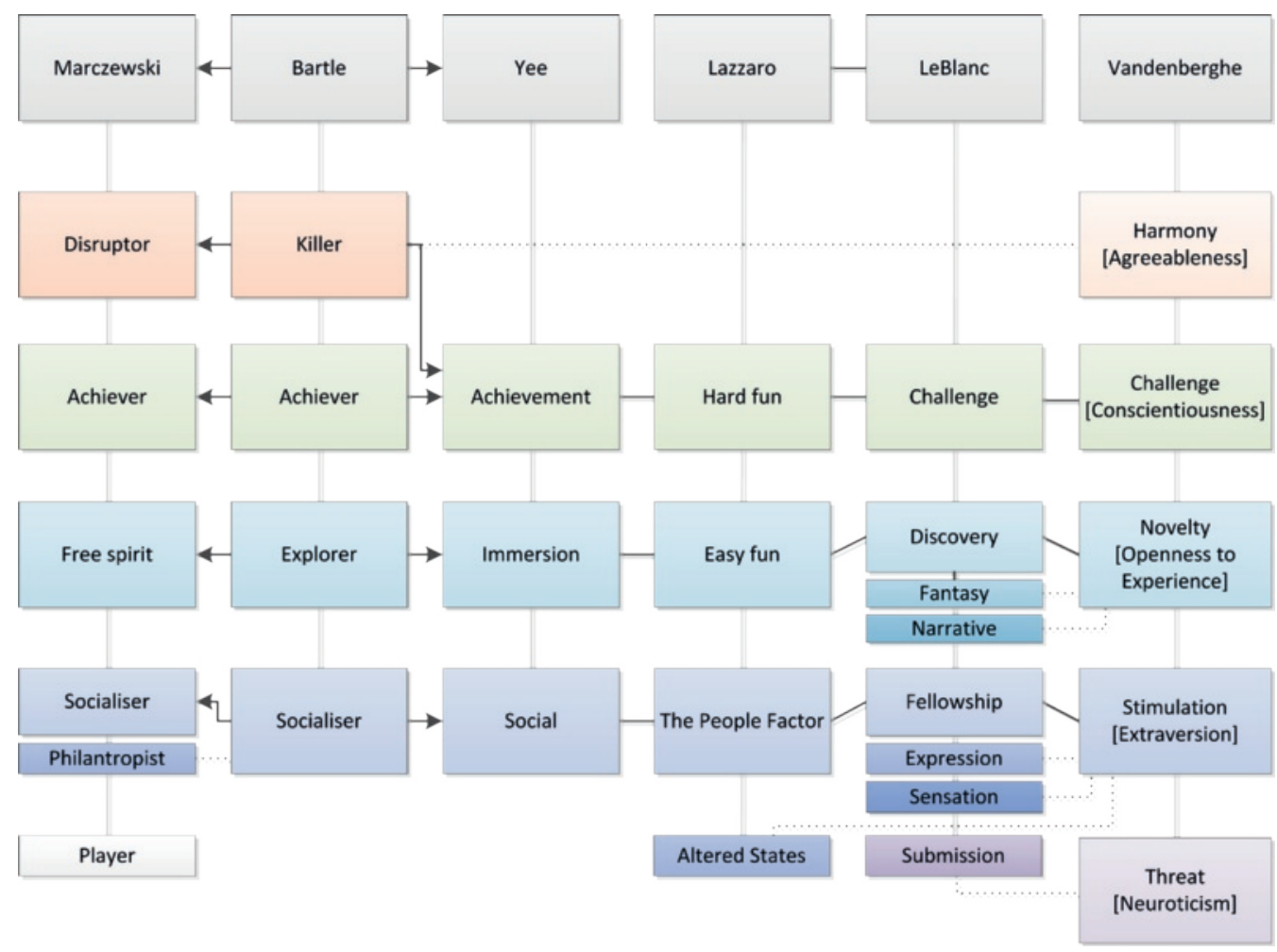

Figure 5 - chart of connections between taxonomies (arrow: direct derivative of, line: high similarity in concept, dots: closely related concepts) 
MMORPGs, and it is not known how suitable they are for application in telemedicine interventions. We can identify many parallels between the models, and we consider that the five domains of play stand out from the rest. Unlike the other models, an individual is not given a singular class label or a combination of those. Instead, a complete character description can be created based on preference for certain aspects or elements of games. What makes this theory even more attractive is that it describes the user based on personality: a universal understanding regardless of age.

\section{3| Discussion}

\subsection{Principal findings}

The first objective of this study was to provide an overview of theoretical frameworks for the application of gamification and of methods for gamification that specifically target the elderly user. Second, we have explored user classification theories, which are needed to gain insight into the user and serve as a tool to effectively tailor content. We have found that current frameworks for gamification rarely target the elderly user. The effectiveness of the use of user classifications for tailored game content is not yet known, neither are there indications for classifying the elderly user with these theories. How can we use these results to systematically design effective gamified telemedicine applications for elderly?

Frameworks for gamification emerge from two main approaches. First, there is a business-oriented approach, with examples of success in practice, using an easyto-apply framework to gamify applications. However, the frameworks from this approach may also be oversimplified, which suffices for marketing purposes but possibly not for long-term engagement needed in telemedicine. Second, frameworks created within academia target for higher causes, such as better education and health outcomes. These frameworks often make use of established theories but are complex, and, at the time of writing, not used in practice. With both approaches, no appropriate framework was found to design gamification for elderly users and application in telemedicine. Therefore, a new framework should be created that is of sufficient depth but applicable in practice and supported by empirical data on its effectiveness. To do so, we would position our future research in academia and take example of the studies presented within this approach. Just like these authors $[32,34,40]$, we would aim for qualitative, long-term engagement and focus on stimulating intrinsic motivation.

Our study showed two approaches for user classification theories: archetypes, where classes are user types with associated preferences, and reasons to play, where classes are based on attributes that describe the user preference. None of the found taxonomies seem to be applicable in telemedicine for elderly users due 
to the very different context and audience for which they have been developed and the fact that we are not familiar with the use of these taxonomies in practice. However, a high level of understanding of the target group will greatly contribute to designing effectively engaging content. This can be achieved by a taxonomy for game design specifically for elderly users. Creating such a taxonomy and corresponding game content can be difficult, since older adults may relate to video games differently than younger users because they might not be able to draw from earlier experience with video games. To create such a classification, it would be most desirable to observe the behaviour of intended users in games, but the scarcity of elderly gamers (and limited availability of games for elderly) does not provide a sufficiently representative subjects for the whole target group.

Although from the taxonomies found none seem directly suitable for creating our future framework, the five domains of play model [48] exceeds the stereotypical classes of the other models by providing a detailed insight and overview of motivations users may have. The model provides an overview of both player and preferences Iwhere others use for example game genres, which are ambiguous, not clearly outlined, and differing for each producer of video games) and is moreover based on a universally applicable psychological concept which may help in overcoming the particular challenge of mapping a group of users onto a taxonomy who have not been exposed to games at a young age. Therefore, we believe the model by Vandenberghe advances on earlier classifications making it unique and worthwhile to explore further for use in game design for elderly users.

Advantages of creating a framework within the academic approach are the possibility of using solid scientifically established theories and incorporating existing motivational theories and instruments that relate to the objective of gamification to motivate and engage. Serious games and exergames for elderly users [72,76] were not included in our study because our present focus is on improving adherence to existing health interventions by means of gamification, and serious games are full games which require a different approach. However, gamification in persuasive (game) design [77-79] or vice versa and gamification for behaviour change [80] [8 1] deserve to be explored. Furthermore, because a well-designed game concept is essential for creating a motivating experience for the user, relevant game design principles that consider the aspect of experience on engagement such as flow $[82,83]$, immersion [50], and customisation [8] can prove useful in reaching our goals. Furthermore, we emphasise the necessity of a good game design concept to successfully gamify an application for engagement. The framework we aim to develop in the future should always leave room for the creative process that is involved. We may be able to predict the preference of a user for different types of content but how content is then designed according to these preferences to appeal to the player could be more art than science. 


\subsection{Conclusion}

In conclusion, we suggest developing a framework for gamification that is based on solid scientific foundations and includes a user classification that specifically assesses the elderly user. We base this classification on the five domains of play model that predicts the existence of a relation between preference for game content and personality. In a study, we need to explore this relation as well as opportunities for use for the intended target group and context. When we know more of these aspects, a gamification framework can be developed by which the classification of the elderly user is used to effectively create tailored, engaging game content. Subsequently, the framework needs to be put to practice and evaluated for empirical support of its effectiveness.

\section{Abbreviations}

ICT: information communication technology

FFM: five factor model

MMORPG: massive multiplayer online role-play games

MUD: multiuser dungeon

MOOC: massive open online course 


\title{
Chapter $3 \mid$ Exploring personality
}

and game preferences

\author{
in the younger and \\ older population: \\ a pilot study
}

In: Proceedings of ICT4AgeingWell (ICT4Awe). 2015. 


\section{Abstract}

Aim Engagement in gamified applications can be increased by effectively meeting end-user preferences for game content. To design this tailored content insight in user preferences is necessary, obtained from user classification models. This pilot study aims to explore the hypothesised relation between personality traits and preference for game characteristics that is the basis for a new user classification model, deduced from the Five Domains of Play theory.

Methods An online questionnaire consisted of the 10-item Big Five Inventory to determine personality, and five questions on the preference for game examples to determine game preference.

Results 216 participants completed the questionnaire $(M=39$ years, $S D=17)$. For the group of participants younger than 60 , four out of five personality traits significantly correlate with their corresponding game preference domains $(r=0,13$ $0,30, p<0,05)$. For the participants older than 60 , no significant correlations were found.

Conclusion Personality and game preferences are weakly related in persons younger than sixty years old, while no relation was found for the older participants. For the latter, this may be due to a lack of gaming experience. We therefore propose to extend research towards a field study by providing actual games to play on beforehand.

\section{1| Introduction}

Demographic changes, such as population growth and longer life expectancy, increase the burden on the healthcare system. Telemedicine can alleviate this burden by enabling professionals to provide care at distance in patients' daily environment [1]. A group that can particularly benefit from telemedicine is the older adult [2]. Telemedicine supports them in maintaining a healthier lifestyle that maintains autonomy, independence and quality of life. Adherence to telemedicine interventions is related to improved health outcomes [3], but is low and decreasing over time [4][5]. A possible explanation for the drop in adherence is the lack of motivation given by a professional in face-to-face contact, or plain boredom. Gamification is identified as a possible strategy to increase adherence by adding the motivational pull that (video) games inherently have, although it is unknown how to apply gamification to produce the needed long-term engagement in telemedicine. This is particularly the case for the older adult, since their preferences are not known from being an unlikely consumer of modern video games. Therefore, there is a need for methods to create gamification for telemedicine solutions, which are tailored to the end-user preferences for optimal engagement. 
User engagement, which is key to adherence, is known to significantly increase when preferences of the user are effectively met [6][7]. Our previous research showed several classifications, to categorise both younger and older users [8], based on for example in-game behaviour or gaming motivation. Examples include Bartle [9], Yee [10] and LeBlanc [1 1]. These taxonomies are designed for use in a specific application, such as enterprise gamification or massive multiplayer online roleplay games (MMORPGs, for example World of Warcraft). It is unknown if these are suitable for application in gamification design for telemedicine solutions. Furthermore, we foresee that older adults demand a different approach, as we also know is the case with many exergames [12], but none of the classifications presented target the older user specifically.

The Five Domains of Play theory (5D) [13] could potentially be suitable for use in telemedicine context. It stands out from the other classifications mentioned as it creates a profile of user preferences, rather than a singular label, based on personality which is an understanding applicable to all ages. According to this theory, people are motivated similarly for playing games as they are in daily life or look to express a particular part of personality that is unsatisfied in real life. The 5D theory translates the five factors of the Five Factor Model of personality (FFM) into five aspects of gaming motivation i.e. game content characteristics (table 1). According to the theory, the score of the personality trait correlates with the score of the translated game domain: a high score on Openness to Experience would mean a preference for a game with high Novelty game content characteristics. As far as we know this relation has not been investigated.

\section{Table 1 - The Five Factor Model of personality and the Five Domains of Play model, including personality/game preferences for both extremes (deduced from Vandenberghe, 2012 [13]).}

\begin{tabular}{|c|c|c|}
\hline low score & $\begin{array}{l}\text { FFM factor } \\
\text { 5D domain }\end{array}$ & high score \\
\hline $\begin{array}{r}\text { Cautious, predictable } \\
\text { Conventional experiences }\end{array}$ & $\begin{array}{c}\text { Openness to Experience } \\
\text { Novelty }\end{array}$ & $\begin{array}{l}\text { Inventive, curious } \\
\text { Open, imaginative experiences }\end{array}$ \\
\hline $\begin{array}{r}\text { Careless, impulsive } \\
\text { Low difficulty, contentment }\end{array}$ & $\begin{array}{c}\text { Conscientiousness } \\
\text { Challenge }\end{array}$ & $\begin{array}{l}\text { Efficient, organised } \\
\text { High difficulty, achievement }\end{array}$ \\
\hline $\begin{array}{r}\text { Reserved, solitary } \\
\text { Single-player, slow pace }\end{array}$ & $\begin{array}{l}\text { Extraversion } \\
\text { Stimulation }\end{array}$ & $\begin{array}{l}\text { Energetic, outgoing } \\
\text { Multi-player, excitement }\end{array}$ \\
\hline $\begin{array}{l}\text { Analytical, detached } \\
\text { Violence, competition }\end{array}$ & $\begin{array}{c}\text { Agreeableness } \\
\text { Harmony }\end{array}$ & $\begin{array}{l}\text { Friendly, compassionate } \\
\text { Cooperation, altruism }\end{array}$ \\
\hline $\begin{array}{l}\text { Confident, secure } \\
\text { Cheerful, calm }\end{array}$ & $\begin{array}{c}\text { Neuroticism } \\
\text { Threat }\end{array}$ & $\begin{array}{l}\text { Nervous, sensitive } \\
\text { Stressful, hostile }\end{array}$ \\
\hline
\end{tabular}




\section{Question 1}

Please indicate where your preference lies between these games and their characteristics. Which game do you find most satisfying to play?

A: Conventional games, games based on real world events
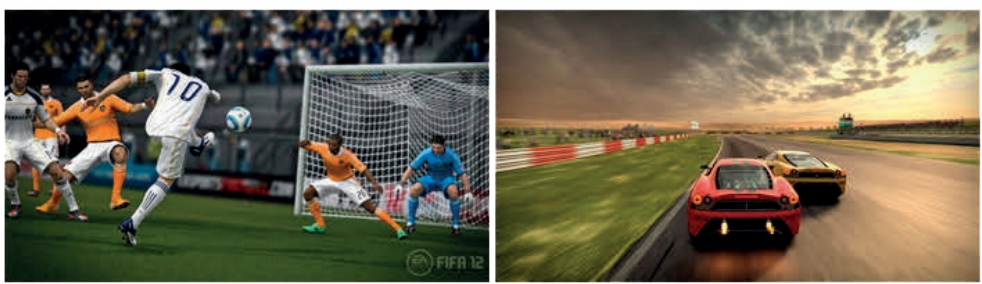

B: Games that are imaginative or artistic, adventurous, open world, fantasy
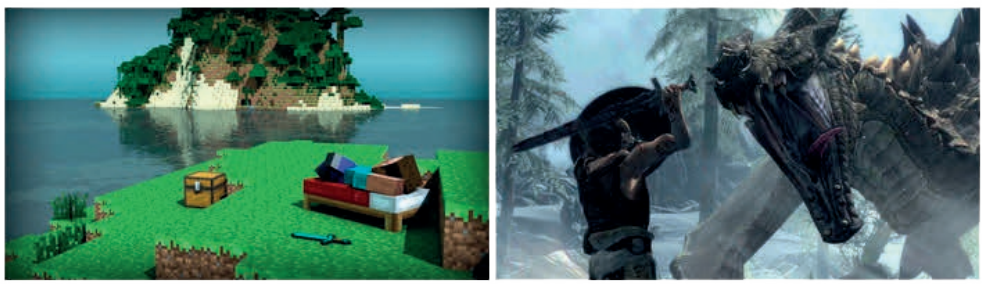

Could you indicate your preference towards these games?

\begin{tabular}{|c|c|c|c|c|c|c|c|c|}
\hline $\begin{array}{l}\text { I find A much } \\
\text { more satisfying } \\
\text { (1) }\end{array}$ & (2) & $\begin{array}{l}\text { I find A } \\
\text { slightly more } \\
\text { satisfying } \\
\text { (3) }\end{array}$ & (4) & $\begin{array}{l}\text { I find } A \text { and } \\
\text { B eventy } \\
\text { satisfying } \\
\text { (5) }\end{array}$ & (6) & $\begin{array}{l}\text { I find B } \\
\text { slightly more } \\
\text { satisfying } \\
(7)\end{array}$ & (8) & $\begin{array}{l}\text { I find B much } \\
\text { more satisfying } \\
\text { (9) }\end{array}$ \\
\hline 0 & 0 & 0 & 0 & 0 & 0 & 0 & 0 & 0 \\
\hline
\end{tabular}

Figure 1 - Question 1 on game preferences (excerpt: Novelty)

So far, studies present inconsistent results on predicting the effectiveness of the application of personality in gaming [14-16]. In one study, personality traits have been related to preference for game genres and low predictive capability was found [17], while another study shows a significant correlation between personality and game genres [18]. Game genres however do not describe explicit game features and may not give an accurate indication of game preferences [19].

If a classification based on the 5D theory indeed appears to be viable, it can give us insight in the user that is essential to design tailored content. Moreover, it provide us with a method to dissect games into distinctive elements. There is a need for a reliable classification of telemedicine end-users according to their preferences, to aid the development of the tailored game content that is essential for creating 
engaging applications through gamification. As a first step toward creating tailored game content, we investigate the hypothesised existence of a relation between personality and game preference based on the 5D theory, for the younger as well as for the older adult.

\section{2| Methods}

\subsection{Participants, materials and measures}

Participants were recruited through Facebook and via e-mail to colleagues and other acquaintances, who forwarded the request to others as well. Furthermore, Alifa, a local social community service centre in Enschede, the Netherlands, sent requests to its older volunteers.

Measures were taken by means of an online questionnaire, created in SurveyMonkey and accessible through a URL. The questionnaire was available both in English and in Dutch. Age and gender were asked first. Play frequency was determined by the question 'How often do you (approximately) play games?' (answer possibilities Daily, Weekly, Monthly, Once every 6 months, Once a year or less). Play frequency was split into two groups: 1) frequent (weekly or more, answers: daily, weekly) and 2) non-frequent (montly or less, answers: montly, once every six months, once a year or less).

Game preference was determined through five questions, one for each of the five domains described by the 5D theory (table 1). In each question, the game content is described through an explanation and depicted examples of a domain's extremes. The participant indicated his or her perceived level of satisfaction (nine-point scale) from the proposed game content (fig. 1). Personality was measured subsequently, by means of the 10-item Big Five Inventory (percentage from 5-point scale). This short version reaches adequate levels in terms of convergence with an established instrument, test repeatability and reliability [20-21]. The FFM model suggests a normal distribution of scores (ranging from $\mathrm{O}$ to 100 with an average score of 50 on each factor).

\subsection{Statistical analysis}

Data analysis was performed using Statistical Package for Social Sciences (SPSS v.22). Age was partitioned in 1) younger than $60(<60)$ and 2) 60 or older ( $\geq$ 60). We define an age threshold assuming that people aged 60 and up have less affinity with technology. First, the data for each person was sorted for the five factors of the FFM model (abbreviated P, from personality) and the five domains of the 5D model (abbreviated $G$, from game preference), together with their gender, age and play frequency. Then, data distributions of the five factors and the five domains were analysed using the Shapiro-Wilk test, descriptives of skewness and 


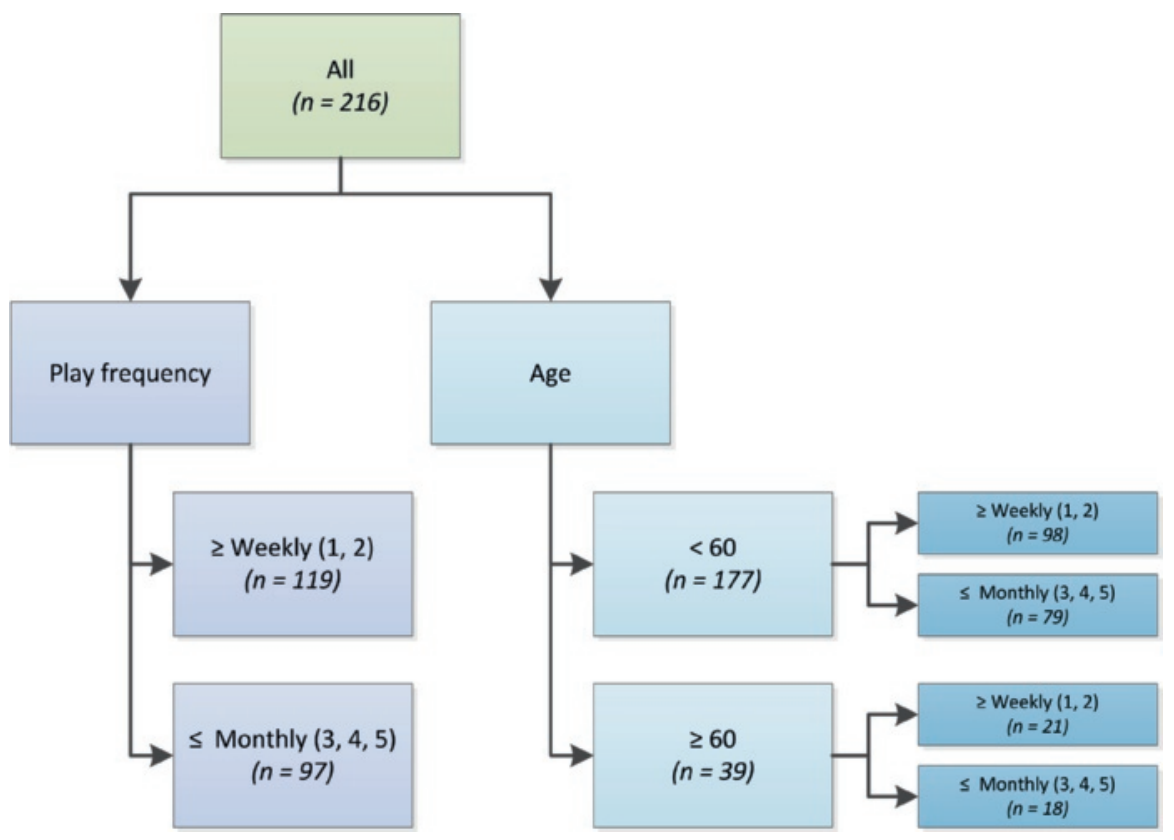

Fig. 2 - Overview of participants

kurtosis, boxplots and histograms. There were no outliers found in analysis of the boxplots. All factors and domains were found non-normally distributed, even after data transformation was applied $(\log 2, \log 10$, sqrt, $\times 2)$. Hence, correlations were explored with Spearman's Rho (rank correlation coefficient, appropriate for ordinal variables), for all groups, using a significance level of $\alpha=0,05$. Correlations between the factors and domains as intended by the models were studied IOpenness - Novelty, Conscientiousness - Challenge, and so on). For the subgroups $<60$ and $\geq 60$, correlations not intended by the models, between all factors and domains, were studied as well. We consider correlation strength as the predictive value of the personality characteristic for the preference on the matching game preference domain. Correlation strength is interpreted as follows: $r< \pm 0,1$ is little or no correlation, $\pm 0,1 \leq r< \pm 0,3$ is a weak relation, $\pm 0,3 \leq r< \pm 0,5$ is a moderate relation and $r \geq \pm 0,5$ a strong relation.

\section{3| Results}

\subsection{Participant characteristics}

In total 243 persons filled in the questionnaire, of which 216 fully completed. The Dutch version was used in $67 \%$ of the cases. The age range was 16 to 81 years old, $66 \%$ of participants were male ( $n=143), 34 \%$ female ( $n=73)$. In fig. 2, an overview of participant characteristics is given. 
Mean values of scores per age group on personality and game preference can be seen in table 2. Considering an expected average of 50 out of 100, the scores on Openness (72 out of 100) and Conscientiousness (66 out of 100) are relatively high. The mean values for personality scores of the different age groups are close together. The older participants score lower on Openness and higher on Conscientiousness than the overall average. Differences are also found between the groups of frequent and non-frequent players.

The mean values for game preference do show great variety, particularly the scores of the $\geq 60$ group (range 24-62). While it seems that personality only has minor changes for the older adult, game preferences show different results compared to the younger group. Especially on the Novelty domain, which implies that the older player prefers games that resemble the real world or familiar. The older adult prefers a lesser amount of Challenge than the younger group, while being more Conscientious. Also, a lower level of Stimulation and Threat are preferred by the older adult, as well as a somewhat higher level of Harmony.

\subsection{Correlations between personality and game preference}

When exploring the intended correlations between personality $(P)$ and game preference $(G)$, we find the following correlations, presented in table 3. No strong correlations were found $(r \geq \pm .5)$, correlations range from little or none at all to moderate. For all participants as well as in both subgroups, a significant correlation between Agreeableness and Harmony is absent.

From the overview of correlations between personality and game preference Itable 3), we notice weak significant correlations between four out of five personality factors and their antagonist in the game preference model for all participants and for the group $<60$ years old. In these groups, we find a negative significant correlation for Conscientiousness-Challenge, which was not dictated by the model. For frequent players, the preference for Novelty seems stronger related to Openness than for non-frequent players. For non-frequent players, Extraversion and Stimulation are significantly correlated, which is not the case for frequent players. For the $\geq$ 60 group, a moderate, insignificant negative correlation between Neuroticism and Threat is found for non-frequent players.

Exploring all possible correlations between personality and game preferences for the group of age $<60$ (table 4), we notice multiple significant correlations outside the factors as intended (shaded). A moderate, significant correlation exists for Openness to Experience and Challenge. Agreeableness was not found to be related to preference within the Harmony domain and also does not show any (significant) correlations with other game preference aspects. Other factors, Openness to Experience, Conscientiousness and Extraversion, do seem to influence the preference for content within this domain. Stimulation is correlated only with 
Table 2 - Mean values of personality and game preference scores ( $M=$ mean value, $S E=$ standard error), all $n=216$, $<60 n=177, \geq 60 n=39$

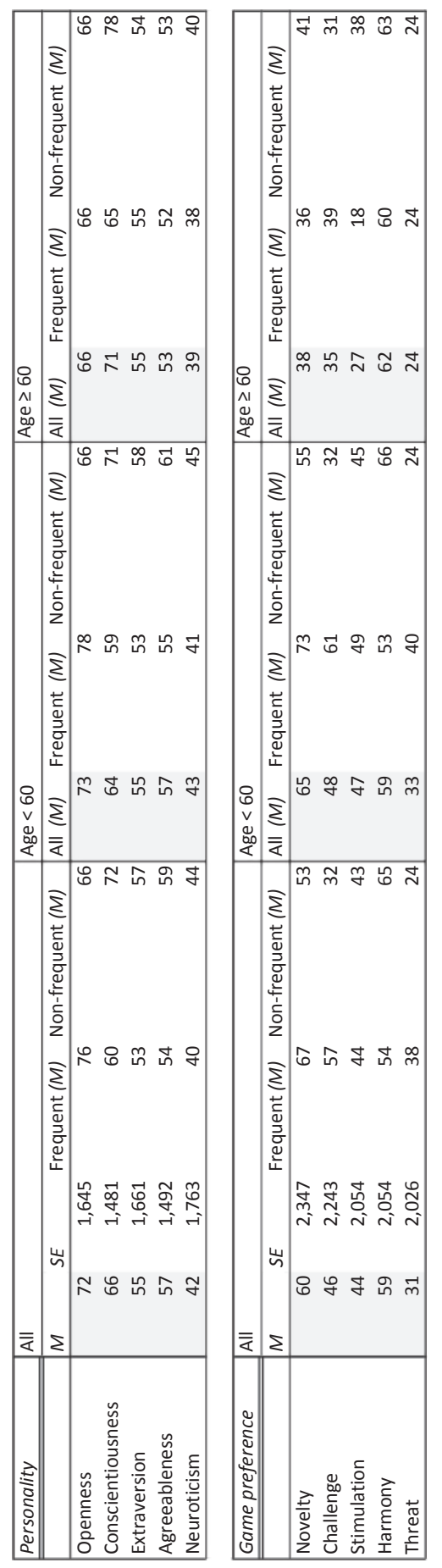

Table 3 - Correlation coefficients and significance between personality and game preferences

\begin{tabular}{|c|c|c|}
\hline $\begin{array}{l}0 \\
0 \\
11 \\
0 \\
00 \\
0\end{array}$ & 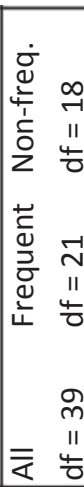 & 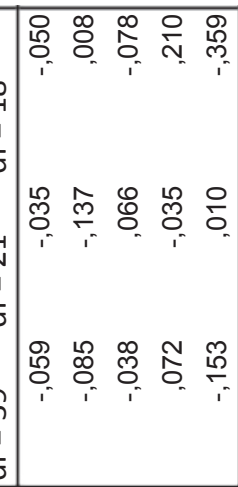 \\
\hline $\begin{array}{l}0 \\
0 \\
v \\
0 \\
00 \\
<\end{array}$ & 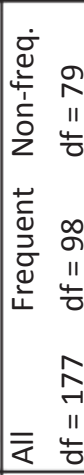 & 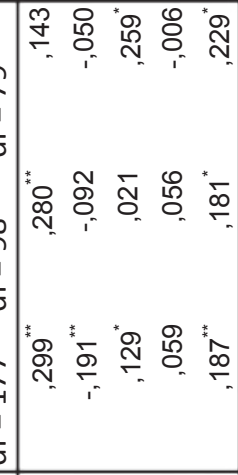 \\
\hline & 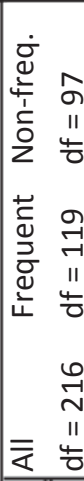 & 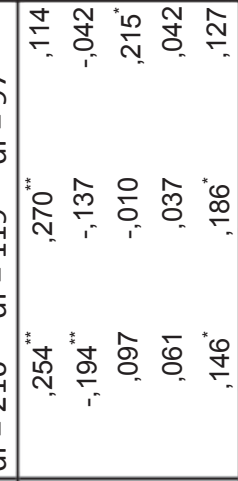 \\
\hline & 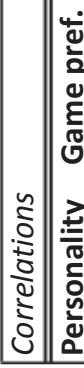 & 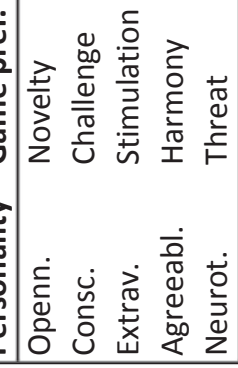 \\
\hline
\end{tabular}


Extraversion, which is exactly how it was intended by the 5D model. Lastly, there is a significant positive correlation for Neuroticism with Threat. Exploring all correlations for the group of $\geq 60$ years old (table 5), we only find a moderate, significant correlation for Agreeableness and Stimulation.

\section{4| Discussion}

In this study, the relations between personality factors and the preference for game content based on the Five Domains of Play model were examined, in search for methods to use in the tailoring of game content to the user for the effective use of gamification. Our most important findings are the presence of correlations between personality and game preference for people younger than 60 years old, and the absence of significant correlations for people aged 60 and up.

Personality seems to give an indication of game preferences according to the theory for participants younger than sixty years old. Four out of five relations as they were intended by the original models correlate significantly, although weakly. This could mean that the model can be used to determine game content for certain user groups. A refinement of the game preference domains should be made in order to deal with ambiguity and increase internal consistency. For example, Agreeableness was not found to be predictive for preference within the Harmony domain as the model suggested which is possibly due to the ambiguity of the Harmony domain, seemingly showing contradictory traits for scores on both sides of the domain spectrum. Also, both competition and violence are supposed to correspond with a low score on Agreeableness. Another finding is that a negative relation between Conscientiousness and Challenge exists, which is inconsistent to the model and may imply that a highly conscientious person would prefer an unchallenging game rather than the opposite. Our findings suggest that a more elaborate questionnaire is needed in future studies to deeper examine the underlying facets that both personality traits and game preference domains consist of, so that these details are not covered by an overall domain score.

No relations between personality and game preference were found for participants of sixty years and older. We expect that the older participants were not able to relate to the game content in the questionnaire to the same extent as the younger group. Older adults, although increasingly interested in video games, have a much different frame of reference than people of later generations [22] from being inexperienced or unaware of the variety of games currently existing. There is a possibility that preferences of the older group are somewhat more uniform because of this awareness. The mean on the Novelty domain for this group is much lower than expected when compared to the Openness to Experience score. This implies that the older player prefers games that resemble the real world or possibly 
Table 4, 5- Overview of all correlations between personality (P) and game preference (G) for participants of $<60$ and $\geq 60$
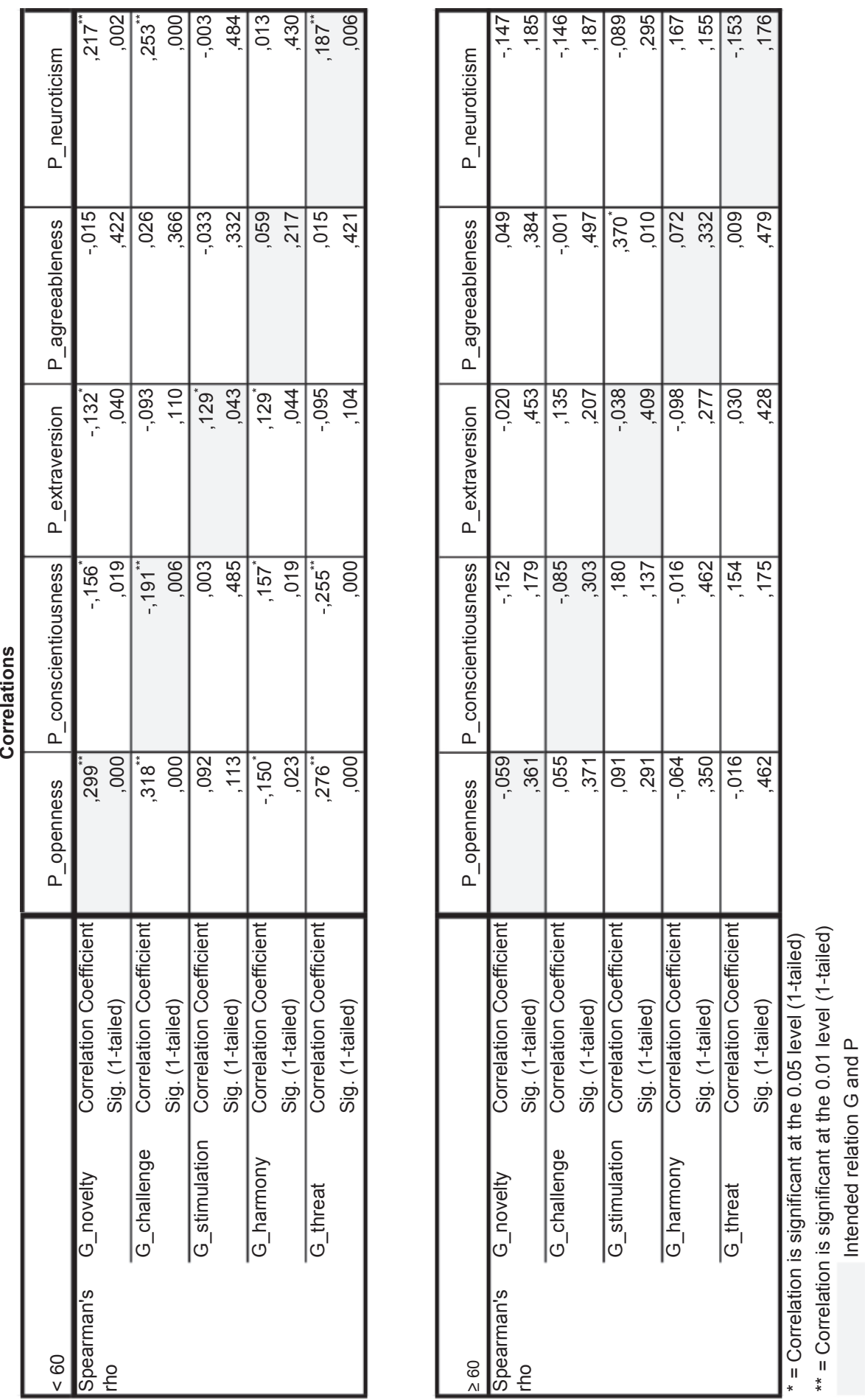
more traditional games as these are familiar for this target group, such as card and board games. The older participants score lower on Openness and higher on Conscientiousness than the overall average, which is according to expected change in personality from ageing [23-24].

This study provided a first exploration of using personality as a method to tailor game content for the effective use of gamification. In comparison to previous studies [14-18], our study can neither offer solid statements on the usefulness of applying personality in gaming. The correlations we have found between personality traits and game preferences, expressed in five domains, are not convincing enough to do so. A limitation of this study is that both models were assessed in a simplified form; the used BFl-1O provides less in-depth information on psychometric measures than a full FFM inventory.

For future studies we would therefore recommend to investigate personality and game preference using a more detailed questionnaire, looking deeper into the six facets for each FFM factor and possibly corresponding factors for each 5D domain to gain a better understanding of both. We see a particular need to refine and investigate the domain Harmony, in which both violence and competition are captured. Furthermore, it is unknown if the correlating factors also determine the choice for certain games, and which factors play the most important role in effectively tailoring game content to the user. We would recommend a different study setup to evaluate the older user, in which the frame of reference is presented to the user by means of actual games previous to analysing their game preferences.

\section{5| Conclusion}

This study investigated the relations between personality and game preference by means of an online questionnaire, in order to tailor game content to the needs of individuals or user groups. Results show that several significant correlations exist for persons younger than sixty years old. We conclude that it is possible that personality gives an indication of game preferences for this group. For participants of sixty years and older, no significant relations have been found. Working towards using this knowledge in practice, research should be extended to 1) deeper examine the models used to determine personality and game preference, 2) gain insight in how to assign content corresponding to preference of the user for effective use in gamification and 3) further explore the models used in a field study towards designing gamification for the older adult user. 
In: CHI Play Conference. 2015.

\section{Game preferences
and personality of \\ Game preferences
and personality of older adult users

(n) \\ . \\ Game preferences
and personality of

fon

(n)

.

(n)

(n)

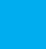$$
\text { . }
$$

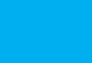

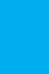

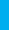
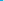


\section{1| Abstract}

To improve engagement needed for long-term adherence to telemedicine services for elderly users, we need methods to design effective and tailored gamification. This study explores the relation between personality lbased on the Five Factor Model) and game preference (based on the Five Domains of Play theory) to enable creation of such a method, by means of a user classification targeting the older adult user. From preliminary results, in a study with 12 participants aged 65-75 years, we observe no significant correlations between personality and game preference. The participants have a strong preference for a game containing novel content. This study provides us with information on the older adult to create a method for developing tailored gamified content based on game preferences.

\section{2| Introduction}

Telemedicine can be particularly beneficial for the older adult [6]. However, adherence to telemedicine interventions is low and decreases over time [2][7]. Therefore, there is a need for strategies to engage older adults in the prolonged use of telemedicine solutions. Gamification - the application of game elements in non-gaming context [1] - may be such a strategy [3]. It is however unknown how to apply gamification for older adult users to produce long-term engagement [9]. By gaining insight in the preferences of users, a method to construct gamification that effectively addresses the (individual) older adult user can be developed. Previous results indicate a relation between personality and game preference for users under 60 years old [10], which may serve as a basis for such a method, by means of a classification of older adult users, as well. Therefore, as a first step toward creating tailored game content, the aim of this study is to explore the relation between personality and game preference specifically for the older adult user.

\section{3| Study set-up}

The study included a personality questionnaire, a game preference questionnaire and a semi-structured interview. In addition, the participants were given tablets with a set of specific games to play with during 5 days.

Personality was measured using the NEO-PI-R Five Factor model (FFM) inventory [4] [5]. The Five Domains of Play theory (5D) was used to measure game preferences [8]. The 5D theory translates the five factors from the FFM that describe personality into aspects of gaming motivation (see table 1, page 27 of this thesis). Each person is ranked on each of the five domains, thereby creating a character description that provides insight in the content that may satisfy the player. 
Table 1 - Selection of five games each participant played on tablet, including total play time of all participants ( $n=12$ ).

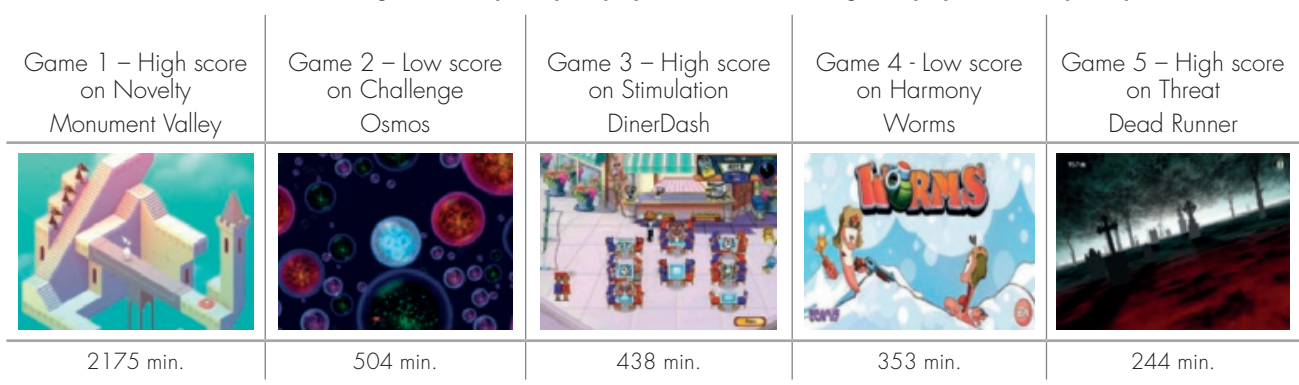

Table 2 - Correlation coefficient $(n=12)$ between personality (P) and game preference $(G)$. *. Correlation is significant at the 0.05 level (2-tailed).

\begin{tabular}{c|r|r|r|r|r} 
Spearman's rho & G_Threat & G_Stimulation & G_Novelty & G_Harmony & G_Challenge \\
\hline P_Neuroticism & 0,07 & $-0,70^{*}$ & $-0,28$ & $-0,10$ & $-0,12$ \\
\hline P_Extraversion & $-0,15$ & 0,40 & 0,03 & 0,12 & $-0,05$ \\
\hline P_Openness & 0,08 & $-0,25$ & 0,25 & 0,09 & 0,77 \\
\hline P_Agreeableness & $-0,29$ & $0,02^{*}$ & 0,27 & 0,19 & 0,06 \\
\hline P_Conscientiousness & 0,22 & $-0,04$ & $-0,07$ & $-0,42$ & 0,23
\end{tabular}

A background on game content was provided to the user by means of five games on a tablet pc, randomly ordered on the home screen, which aided the participant to adequately answer the questions determining game preferences and tracked play time (table 1). Each game corresponded with an alternating extreme in the 5D theory (high Novelty, low Challenge, and so on). After 5 days of freely playing the games of choice, the study concluded with a questionnaire on game preferences and an additional interview.

\section{4| Results}

Twelve people between the age of 65 and 75 years old participated in the study, recruited through a local community centre. The average use time on the total of five games was over 5 hours per participant. Game 1 was favoured by 11 out of 12 participants with a total play time of 2175 minutes (table 2), which is an average of 3 hours per person.

Table 2 shows correlations between the FFM factors (P) and 5D domains (G) as intended by the models (Openness - Novelty, Conscientiousness - Challenge, and so on), which were explored with Spearman's Rho for all users (significance level a $=0,051$. A significant relation between personality and game preference was not observed in the group of older adults in this study, conflicting with earlier obtained results from users $<60$ years old. 


\section{5| Discussion and conclusion}

This study shows that older adult users have a strong preference for a game with high Novelty content, and that significant correlations between personality and game preference were not found. A possible explanation for this observation may be that an extensive experience with video games, only acquired when growing up playing video games, is essential for being able to classify user preferences in a model such as used in this study. Indeed, in our previous study [10] we found indications for the existence of a relation between personality and game preference in participants younger than 60 years old.

When the younger people of this generation have aged, it would be interesting to repeat this study. From our preliminary results we could conclude that personality of the older adult user does not directly indicate game preferences. However, we will further analyse the game preferences of the individual participants - combined with the monitored use time data of the games played with qualitative information from interviews - and create design guidelines that can be translated into tangible design that is tailored to the end user. 

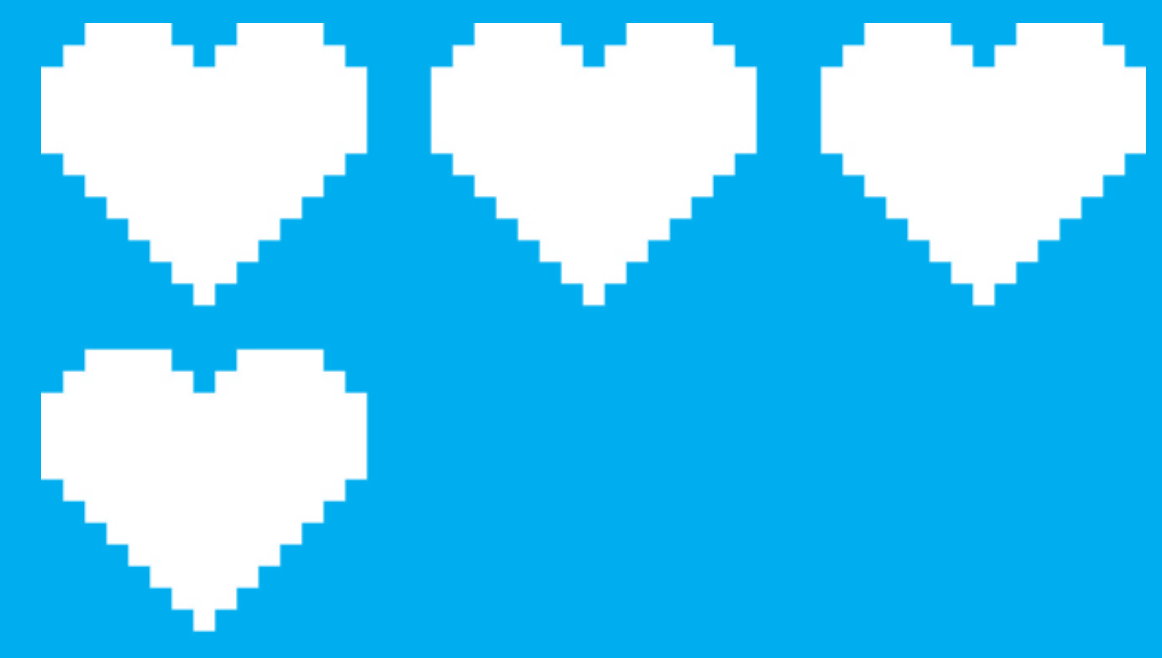

$$
\text { ( }
$$
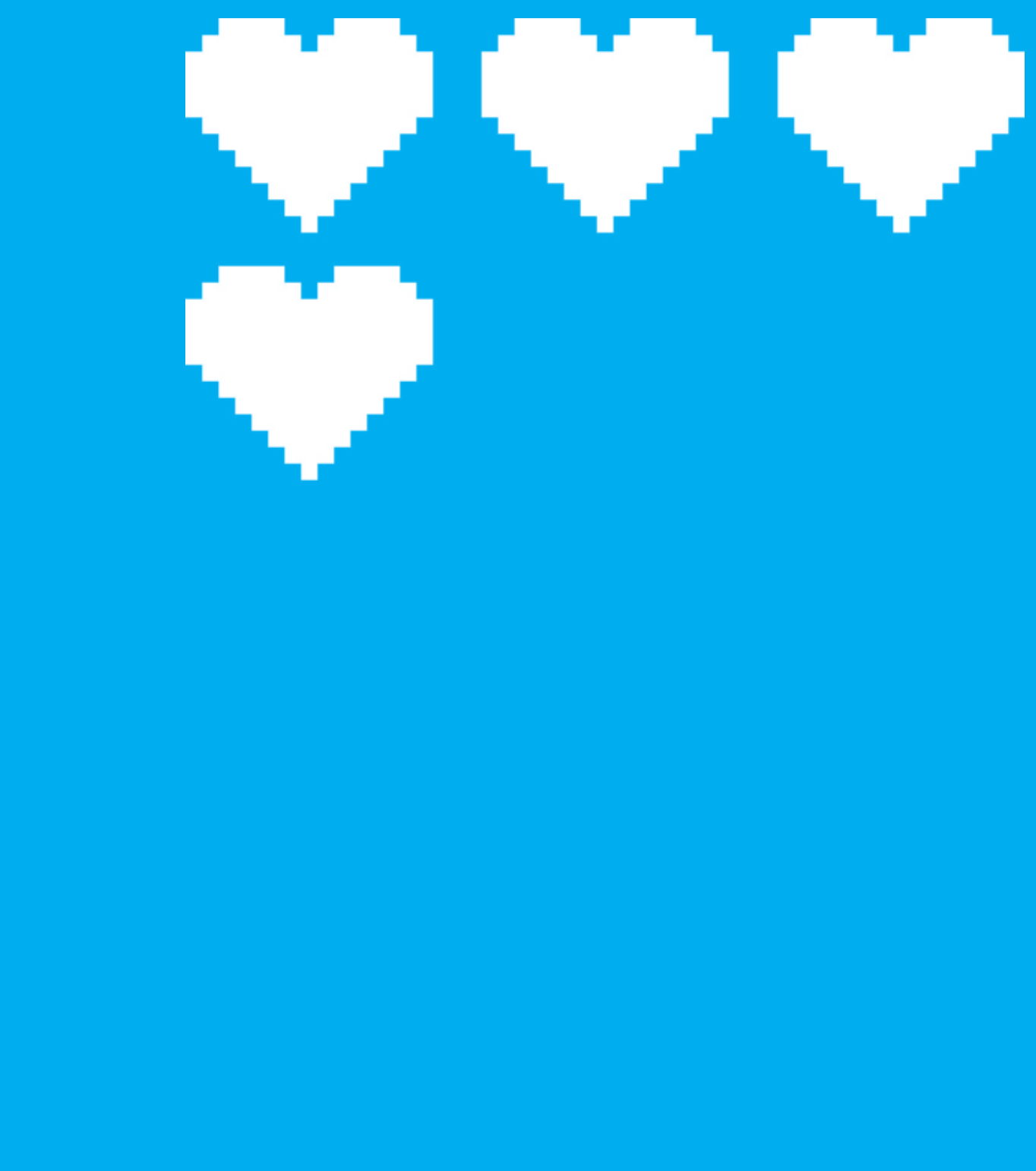

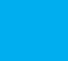




\section{Chapter 4| Mapping game}

preferences of older

adults: a field

study towards tailored

gamified applications

In: Proceedings of NordiCH'1․ 2018. 


\section{Abstract}

Digital applications are a valuable addition to traditional healthcare. An opportunity in retaining motivation to use these applications lies in gamification. To design a gamified healthcare application for a specific target group, such as the older adult, detailed insight in their game preferences is needed. This insight enables us to tailor gamification to the user to make applications more engaging and usable, which is thought to better facilitate underlying healthcare goals. This raises the need for methods to assess these game preferences. Towards such a method, we deploy a classification for game content based on the five domains of play theory.

In this study, 12 older adults played five off-the-shelf tablet games for one week. Preferences were assessed through a questionnaire, log data and semi-structured interview. The resulting overview of preferences was used to make recommendations for future gamification as well as a refinement of the classification.

\section{1| Introduction}

Digital healthcare applications - telemedicine, the use of IT solutions that enable medical professionals to remotely provide care to patients in their daily environment [1] - can contribute to the alleviation of the increasing demand for care [2] that is caused by a rapidly changing socioeconomic structure [3]. As there is a high old-age dependency ratio in Western countries [4], telemedicine applications that enable people to maintain their autonomy and independence by adopting a more active and healthy lifestyle are particularly valuable [5]. An issue to overcome in the use of these applications in practice is a rapidly decreasing adherence that occurs after several weeks of use [6][7], which is possibly caused by loss of interest and a drop in motivation. As such, there is a need for strategies to motivate people to use digital healthcare applications on the long term.

Gamification, the application of game elements to retain motivation in the use of applications outside entertainment, may be such a strategy, as it can increase engagement to an application or make otherwise boring routine of exercises more endurable [8]. Older adults increasingly gain access to digital games through the popularity of tablets, and appreciate tablet use in healthcare setting [9]. Furthermore, the positive effects of gamification in digital healthcare for the older adult are demonstrated [10]. However, the majority of research on gamification in healthcare is focused on a younger population [ 11 ] and we are uncertain how to tailor game content for this target group to increase and sustain engagement. Instead of borrowing strategies from practical examples of successful gamification for the older adult, we aim to address the specific preferences for game content the older adult may have, which we can transpose to healthcare applications. 
In this study, we gain insight in the preferences for game content of a group of older adults. Working towards a method to assess these preferences, game content should be made explicit more elaborately than current practice by for example game genre classifications [12]. We therefore explore the use of a classification for game content based on the five domains of play theory [13] and assess preferences by means of a questionnaire. As it is vital that the participant can relate to the game content presented in this questionnaire, we aimed to give participants a basic experience with modern (i.e. recently developed, not based on conventional games) video games. Few older adults are acquainted with such games, thereby lacking background to answer questions about their specific preferences. We therefore supplied the participants with a tablet pc and five off-the-shelf games to be played for a week before assessing their preferences. Next to an initial understanding of the preferences this target group may have, this study gives us insight in the potential of the classification to assess and visualise game preferences of older adults, which can aid the development of tailored game content essential for creating engaging applications.

\section{2| Background - game content in five factors}

At the origins of the development of a new classification for game content lies the five factor model [14], selected for its preferred use in other studies about game preferences [15-18] and comprehensiveness, predictive powers, reliability and universality [19-23]. Following the five factor model, the five domains of play theory semantically translates the original five factors of personality into aspects of gaming motivation [13]. Each of the five domains describes the nature of game content that the player may find satisfying. The domains are continuous variables, which together form a characterisation of the content of a game. Hypothetically, the preferences for these aspects of game content a user may have are in conjunction with personality. The theoretical background of this classification, i.e. the relations between personality and preference for game content were explored by De Vette et al., for adults (including older adults) in general [24], and for the older adult specifically [25]. The latter was done by means of an additional questionnaire on personality that was part of the method of the paper at hand. This analysis has been left out of scope for this article. Even though the relation between personality and game content for the older adult could not be supported by statistical evidence, we find that the classification for game content itself may be suitable for assessing and expressing game preference. The five domains of play theory provides an essential level of depth and comprehensiveness to describe game content that was not discovered in other existing classifications [26]. This is mainly because in most other classifications, characteristics contain singular instead of continuous classes, or are applicable only to a specific context (such as MMORPGs). This results in a categorisation of users along stereotypes, as is often the case in earlier player type 


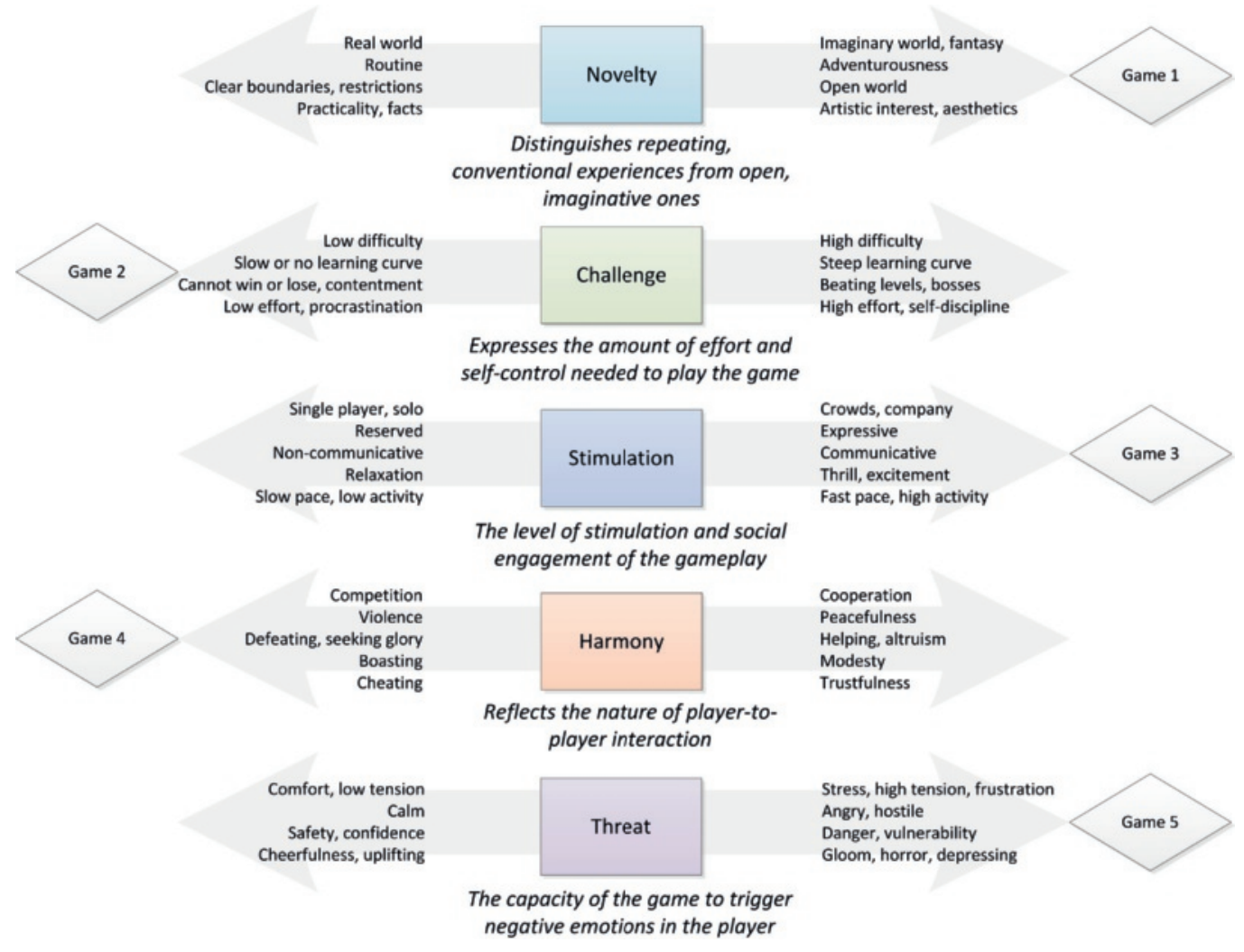

Figure 1 - Game content in five domains

models, or genres, to which the user's favourite games can roughly be assigned to. In this study, we deploy a classification of game content that is a modified version of the five domains of play theory. According to the original theory, each of the five domains has six underlying facets, which are directly derived from the facets of the five factor model. To enable analysis of the game content that may satisfy a user by means of a questionnaire, now that its original assessment method through personality has been omitted, we further elaborated the model by adding semantics to both ends of each domain (fig. 1). Also, some facets of the five factor model were considered not relevant or translatable into game content and have therefore been left out of scope for this study.

The domains enable to 'rate' content based on its novelty, challenge, stimulation, harmony and threat. Each of these domains is characterised on both sides of the spectrum with examples of game content. Oversimplified, the domain novelty, for example, distinguishes between game content that is repeating and predictable, and more imaginative and open. Content characteristics for the one end are the presence of clear boundaries, similarity to the real world and routine. On the opposite side, we find game content that can be described as adventurous, fantastical, artistic or 
aesthetical. The classification can therefore be used to describe the contents of a game, as well as to express the preferences for game content a user may have.

\section{3| Methods}

Participants were given a tablet with five off-the-shelf modern games, and were asked to freely play their games of choice. They were introduced to the device and each game at an intake session. Before the use time, the participant was asked to complete a questionnaire on demographics. The study concluded with a questionnaire on game preferences and an additional interview to discuss the games' attributes on a higher level.

\subsection{Participants}

Twelve people between the age of 65 and 75 years old, six male and six female, participated. Participants were recruited through a local community centre (Alifa in Enschede, the Netherlands), via an internal request to its older volunteers. Inclusion criteria were to have affinity with smartphone or tablet and to enjoy playing games regardless of experience or playing frequency. Participants were excluded when their cognitive or their physical abilities did not allow them to complete the test protocol (severe visual, auditory or cognitive impairment).

\subsection{Materials and measures}

Participants were provided five games installed on a tablet pc (10" screen), randomly ordered on the home screen. A paper manual was provided for the tablet pc. At the intake session, demographics were measured by means of a paper questionnaire. Game preferences were determined by means of a second paper questionnaire, filled in after the use period of a week, in 84 items. The participant was asked to indicate how much satisfaction was derived from playing each game (1-9 numbered VAS scale), followed by questions resulting in domain specific scores (percentages): how satisfying a series of key features were found to be (1-9 numbered VAS scale) and by scoring on agreement on a set of statements on game preference (1-9 numbered VAS scale) without referring to particular games. Playing time for each game was measured in full minutes through an app tracker application hidden in the background. The participant was informed about this measurement. In case of missing data (cut-off point 3 minutes playing time) the participant would be omitted from the results. Previous gaming experience, experience with the five games from the test and motivating and demotivating aspects of each game were discussed in a concluding semi-structured interview. Thematic content analysis was performed on the interview data. Interviews were transcribed by the authors. The themes are the content characteristics from the five domains (fig. 1). The number of times unique participants mentioned these themes as either motivating or demotivating were counted. 


\begin{tabular}{|c|c|c|c|c|c|c|}
\hline 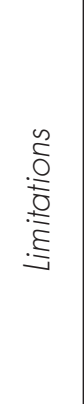 & 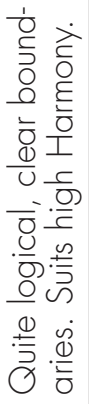 & 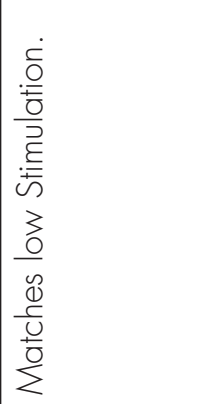 & 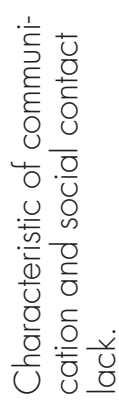 & 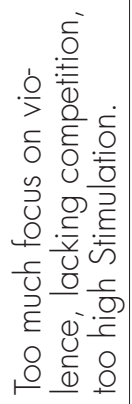 & 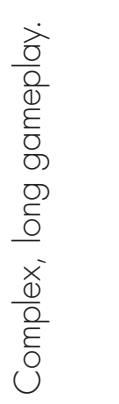 & 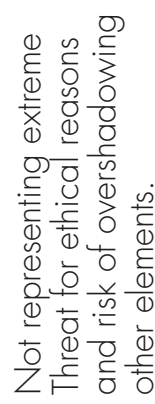 \\
\hline$\frac{\mathbb{2}}{\frac{0}{0}}$ & $\sigma$ & $\begin{array}{l}\frac{\bigcirc}{=} \\
\infty\end{array}$ & 0 & ڤn & $\frac{O}{\nwarrow}$ & $\begin{array}{l}\frac{0}{=} \\
\infty\end{array}$ \\
\hline 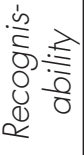 & $\begin{array}{l}\text { ऽ } \\
\text { ऽ } \\
-\end{array}$ & $\begin{array}{l}\simeq \\
= \\
=\end{array}$ & $\stackrel{\circ}{\circ}$ & 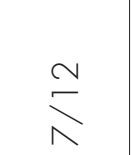 & $\begin{array}{l}\simeq \\
\searrow \\
\simeq\end{array}$ & $\begin{array}{l}\text { ऽ } \\
\searrow \\
\simeq\end{array}$ \\
\hline $\begin{array}{l}\frac{\delta}{0} \\
\frac{a}{2} \\
\frac{0}{0} \\
0 \\
0 \\
0\end{array}$ & 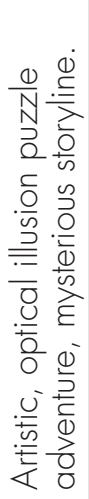 & 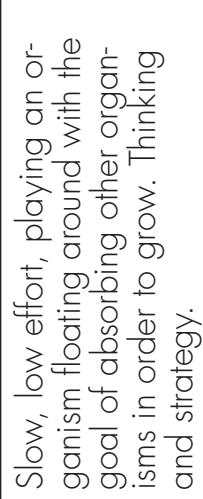 & 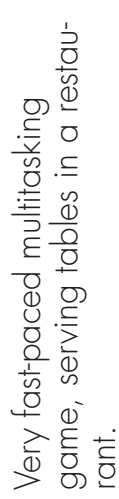 & 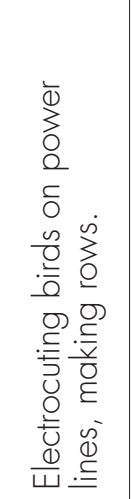 & 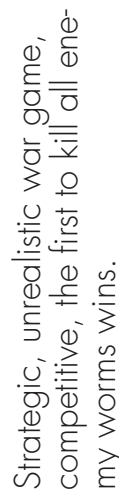 & 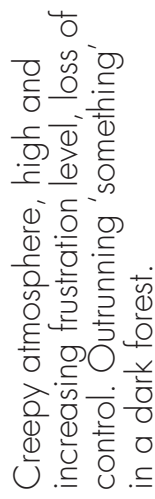 \\
\hline $\begin{array}{l}: \\
\text { ठ } \\
\text { ठ } \\
0\end{array}$ & $\begin{array}{l}+ \\
\frac{a}{10} \\
\frac{0}{0} \\
Z\end{array}$ & $\begin{array}{l}\frac{0}{D} \\
\frac{c}{0} \\
\frac{D}{\overline{0}} \\
\frac{C}{U}\end{array}$ & 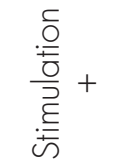 & 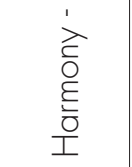 & $\begin{array}{l}\text { ટે } \\
\text { ह } \\
\text { 음 } \\
\text { I }\end{array}$ & 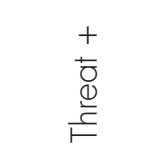 \\
\hline $\begin{array}{l}\frac{1}{2} \\
\frac{0}{0} \\
z\end{array}$ & 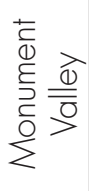 & $\begin{array}{l}\stackrel{\tilde{O}}{\varepsilon_{0}} \\
\stackrel{5}{0}\end{array}$ & 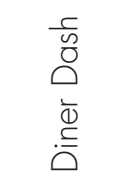 & 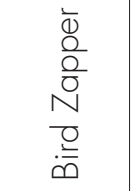 & $\begin{array}{l}m \\
\infty \\
\xi \\
0 \\
3\end{array}$ & 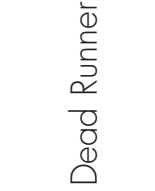 \\
\hline & \# & $\#$ & \# & $\underset{\#}{\stackrel{0}{+}}$ & $\underset{\#}{\stackrel{0}{t}}$ & $\#$ \\
\hline
\end{tabular}

Table 1 - Overview of selected games 


\subsection{Game selection}

An initial selection of five games was made by the authors. All five games are offthe-shelf and generally available, sourced from GooglePlay. They were chosen for strongly representing one extreme of each domain of game content. For the domains Harmony and Threat, we chose games moderately representing this content as not to strike ethical issues and distort measurements. The choice for five games instead of ten for each participant was made to reduce cross-linking between the domains, minimising the load and possible confusion for the participant at the same time.

The selection of games was discussed in an expert session. The 12 experts were male and female researchers with various academic degrees, in the fields of psychology, computer science, biomedical engineering, human movement sciences, human media interaction, health sciences and game design, in the age of 24 to 60 . Their gaming experience ranged from seasoned gamers who play every day to occasional players. The session included a presentation of the underlying theory and classification used, the five games and the rationale for this selection, and a questionnaire. Through this questionnaire, the experts were asked to indicate if they were able to identify the game elements within the selected game as intended (yes/no, please elaborate), to give a mark for suitability of this game to the corresponding five domains trait (score 1 to 10). We set the minimum number of people to properly recognise the game features to 10 out of 12 and the suitability to the model to be rated 7 or higher. Lastly, there was room for remarks on possible limitations, suggestions for alternative games and discussion. An overview is shown in Table 1.

The characteristics of the game 'Bird Zapper' were not sufficiently recognisable for low Harmony, and the domain suitability was rated insufficient. An alternative suggested by the experts, Worms 3 , to better suit the domain extreme's characteristics was chosen. All experts agreed this would be a more representative game (re-rated afterwards).

\section{4| Results}

\subsection{Participant characteristics}

Among the participants are 8 regular players of video games (once a week to daily), 3 less frequent players (monthly) and one person indicates to be fanatic in board and card games but hardly ever plays videogames. All participants indicate high computer literacy (self-assigned). Their favourite games include Scrabble, Wordfeud, Bridge and other card games such as Freecell and Solitaire, puzzle games and games via Facebook. Four people play online, eight people play offline. The majority of participants indicated to frequently play board games, all of them mentioning social contact as a main reason. 


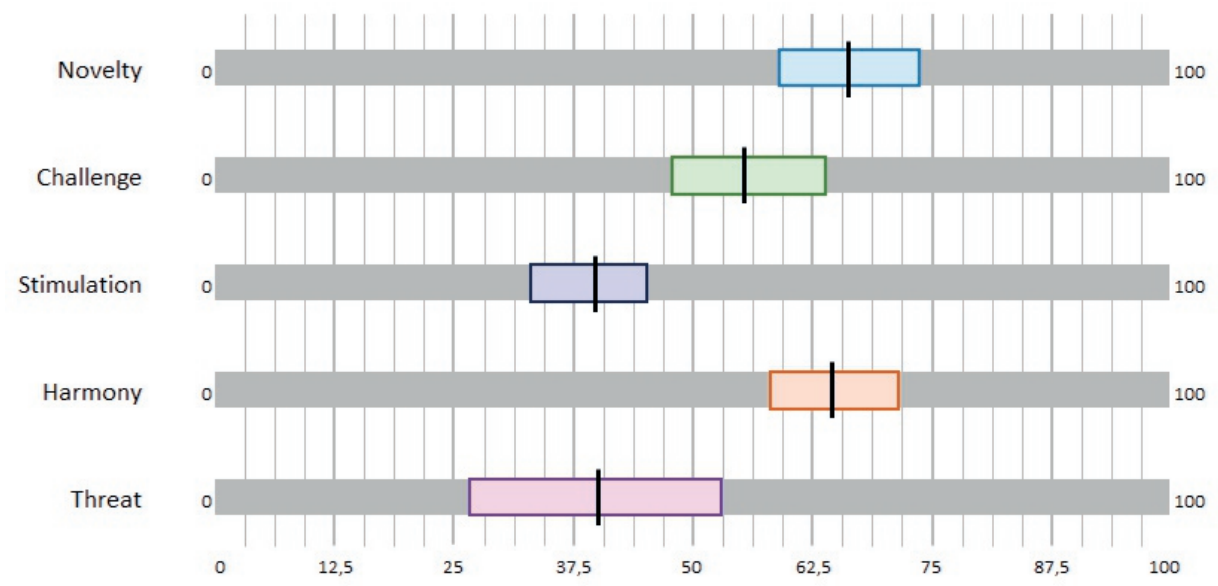

Figure 2 - Game preference, domain scores $(n=12)$, mean and $95 \%$ interval

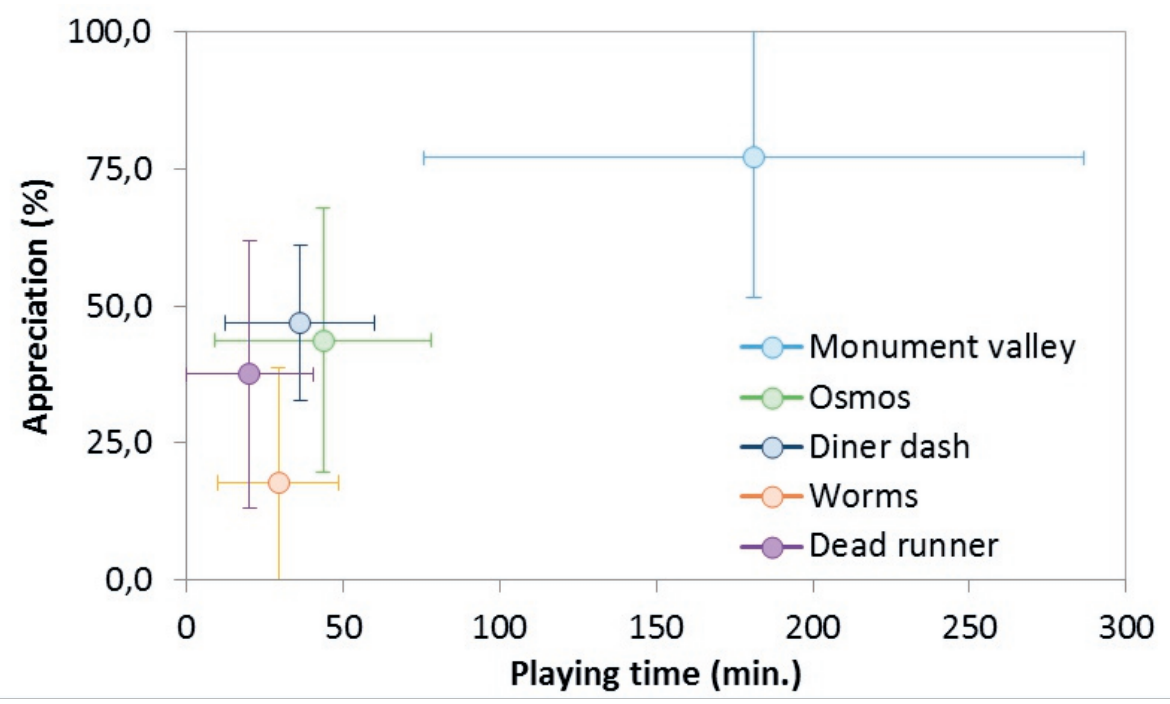

Figure 3 - Playing time (in minutes) and appreciation (in percentages) (mean and std. error)

\section{Domain scores}

Table 2 shows the results on game preferences measured by the questionnaire on each of the seven domains. These scores are also visualised in figure 2, using the mean scores and $95 \%$ interval.

\section{Appreciation of games and time played}

From the questionnaire, we derive the following scores for the appreciation for each game (Table 3). Each value is the average of the score all users gave for the appreciation of the game (highest score Monument Valley, 77.1, lowest score 
Worms $3,17.11$. The average score given for all games is 44.6. The relation between played time of all games (measured through an app in the background) and game preferences of all users in the population is explored through a graph (Fig. 3). All participants played each game for a sufficient amount of time $13+$ minutes) so there are no cases of missing data. Out of a total playing time of 3714 of all users, Monument Valley is played longest (2175 minutes, which is 3 hours on average per person) and Dead Runner shortest (244 minutes).

\section{Interview data}

Interview data were categorised into motivating and demotivating aspects of each game (Table 4), as well as usability remarks that are either limiting (-) or enabling $(+)$ gameplay. Between brackets is the number of participants that made the remark. Participants' favourite games are card and word games, three participants indicate to play mostly modern games online. When asked what the most important motivation was to play these games, competition is valued by 9 out of 12 players, 9 out of 12 players indicate to play mostly to enjoy company regardless of which game is played.

\section{5| Discussion}

In this study, we examined the preferences of older adult users regarding game elements, categorised into five domains, after providing them with a frame of reference for expressing their preferences by means of five modern off-the-shelf mobile games. We gained more insight in the preferences of older adults regarding modern game content, often different from the obvious, and in the classifications' weaknesses.

\subsection{Preferences}

We analysed the scores on the different domains of game preferences. From previous study results $(n=39$, age $\geq 60$ ) [13] we expected that the older user would prefer game elements from the low side of the Novelty domain. This would be in concordance with the games they indicate to play the most, which usually are digital versions of conventional games that they were already familiar with. The score for Novelty from this study however is on the higher side of the spectrum. This is also the case for Challenge. Stimulation, Harmony and Threat are scored to expectations from previous work. Violence is disliked by the majority of participants, as are a depressing surrounding, stress and frustration.

A similar balance is found in the overall appreciation and total playing time per game. Monument Valley, high Novelty, stands out positively; the preference for high Novelty content seems to match the high Novelty game content. Worms, low Harmony, stands out in negative sense; the violence of the game and the 
Table 2 - Descriptives domain scores (scale 0-100, $n=12$ )

\begin{tabular}{|c|c|c|c|c|c|}
\hline & Novelty & Challenge & Stimulation & Harmony & Threat \\
\hline Mean & 66 & 55 & 39 & 64 & 40 \\
\hline Std. Error & 11 & 12 & 9 & 11 & 21 \\
\hline Lower bout 95\% & 59 & 47 & 33 & 57 & 26 \\
\hline Upper bout 95\% & 73 & 63 & 44 & 71 & 53 \\
\hline
\end{tabular}

Table 3 - Appreciation and playing time per game

\begin{tabular}{|c|c|c|}
\hline & Average/game & Minutes/game \\
\hline Monument Valley & 77.1 & 2175 \\
\hline Osmos & 43.8 & 504 \\
\hline Diner Dash & 46.9 & 438 \\
\hline Worms 3 & 17.7 & 353 \\
\hline Dead Runner & 37.5 & 244 \\
\hline Average score/user & 44.6 & - \\
\hline Total time played & - & 3714 \\
\hline
\end{tabular}

disapproval of the elderly may cause the low score on this game. These results must however be viewed in the light of a different amount of time spent for each game for a sense of completion. A discrepancy is found between the time Worms and Dead Runner were played: the score for Worms is lower than for Dead Runner while the latter is played for a shorter amount of time. A playing session on Dead Runner takes seconds to minutes, while a session in Worms may take ten minutes or more to finish. Diner Dash levels take approximately three minutes to finish. Osmos playing time ranges from five minutes, when directly following the objective, to endlessly exploring. In Monument Valley, solving a puzzle may define a feeling of completion, which usually takes a few minutes. The high total playing time of Monument Valley is therefore not explained by a more lengthy playing session.

In the interviews, the (de)motivating elements mentioned for each game are much in line with the opinions of the experts in the process of game selection validation. However, results of this study indicate that we should verify even better if our perspective, that of the designer, aligns with the way the target group receives the content in order to effectively design suitable content. For example, we see that Osmos, a game that we thought was rather slow, was perceived as unpleasantly fast by some. Similarly, it was beyond expectations that even shooting cartoon worms was considered to be extremely negative and violent. Also, the score of the older adult on Threat is higher than expected, but we should consider that the game selected for high Threat, Dead Runner, is rather mild in our eyes. 


\subsection{Usability}

An engaging game requires correspondence with the preferences and abilities of the target group. We find that usability is a threshold in the enjoyment of modern games by older adults. These games are largely inaccessible to this target group, which explains their inability to express their game preferences without presenting a more up-to-date point of reference. The majority of participants remarked in the interviews that games were abandoned when they would become too fast, too much instruction was needed to play, or when working with small visual items or an overload of information at the same time became too fatiguing. According to the participants, the focus of a game should never be on physical agility but on cognitive skills as people feel more challenged in using their brains than their (possibly diminishing) physical skills and reaction speed. Participants remarked that when the game did not involve 'thinking', they were inclined to give up sooner. In addition, they indicated it was necessary for their motivation to always have a clear goal and be able to keep track of their progress. This includes being able to pause and continue at any point in the game. The element of discovery, as part of high Novelty, is appreciated only when the goal is not too abstract, as is the case with Osmos.

Usability is researched and described in several other studies [27-30], on the topics of interface and interaction design and physical and cognitive challenges of the target group. Contributing to this body of knowledge, we carefully recommend the following based on our study results:

- Hints or cheats should be available on request when the player is stuck. Not being able to finish a level or puzzle leads to the user giving up on playing the game, inducing feelings of frustration and incompetence.

- Games should have the function to be stopped and continued at any point in the game. Once the player has the impression that effort is wasted in doing the same thing twice, it is less likely that the game will be picked up again.

- Progression and goals should always be clear to the user. Trial-and-error in general is not an approach many older adults wish to take in games.

\subsection{Profile comparison and design recommendations}

Considering the above, why is Monument Valley the ultimate favourite? We see that the profile of the older adult matches this game's characteristics best on all domains, just as Worms matches worst. Supportive to these findings, in the interviews the characteristics belonging to high Novelty are also mentioned as positively motivating in other games than Monument Valley and even demotivating when absent, for example aesthetics, discovery and fantasy. 
Table 4 - Interview data: motivating and demotivating characteristics of the games played (mentioned by number of participants between brackets, usability remarks positive (+) or negative (-))

\begin{tabular}{|c|c|c|c|c|c|c|c|c|c|c|c|c|c|c|c|c|c|c|}
\hline 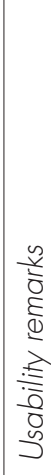 & 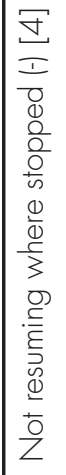 & 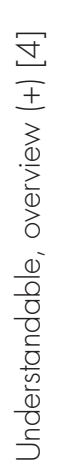 & 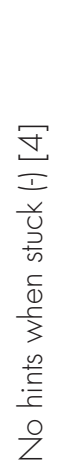 & 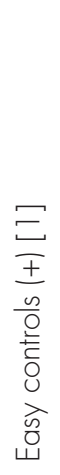 & & & & & & & & 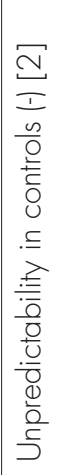 & & & & & & \\
\hline 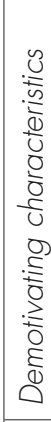 & 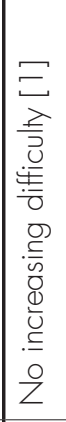 & & & & & & & & & & & 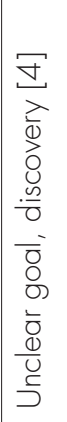 & 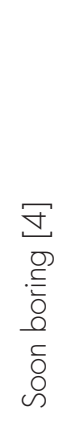 & 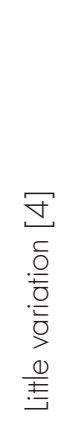 & 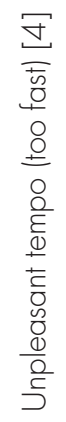 & 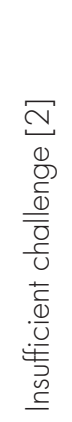 & 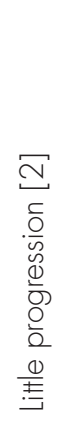 & \\
\hline 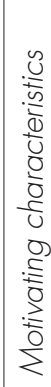 & 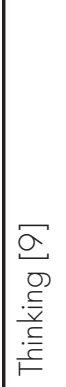 & 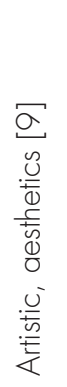 & 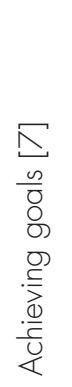 & 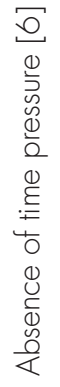 & 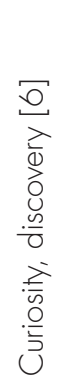 & 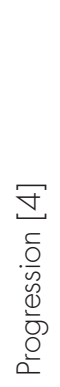 & 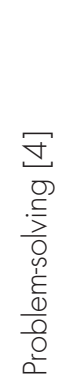 & $\begin{array}{l}\text { 巳 } \\
\text { D } \\
\bar{D} \\
\overline{\bar{D}} \\
\frac{\bar{U}}{U}\end{array}$ & 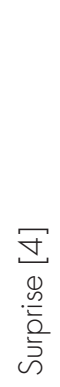 & 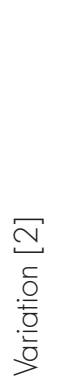 & 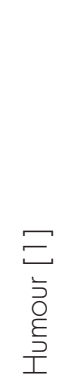 & 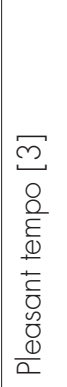 & 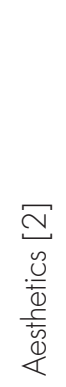 & 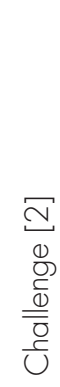 & $\begin{array}{l}\text { E } \\
\text { ठ্े } \\
\frac{0}{0} \\
\frac{D}{\omega}\end{array}$ & 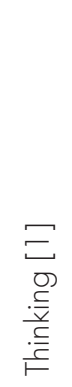 & 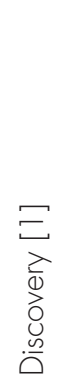 & 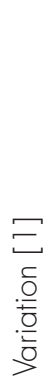 \\
\hline $\begin{array}{l}0 \\
\text { है } \\
0 \\
0\end{array}$ & & & & & & 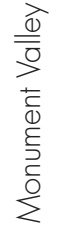 & & & & & & & & & $\stackrel{\sim}{\stackrel{\infty}{\infty}}$ & & & \\
\hline
\end{tabular}




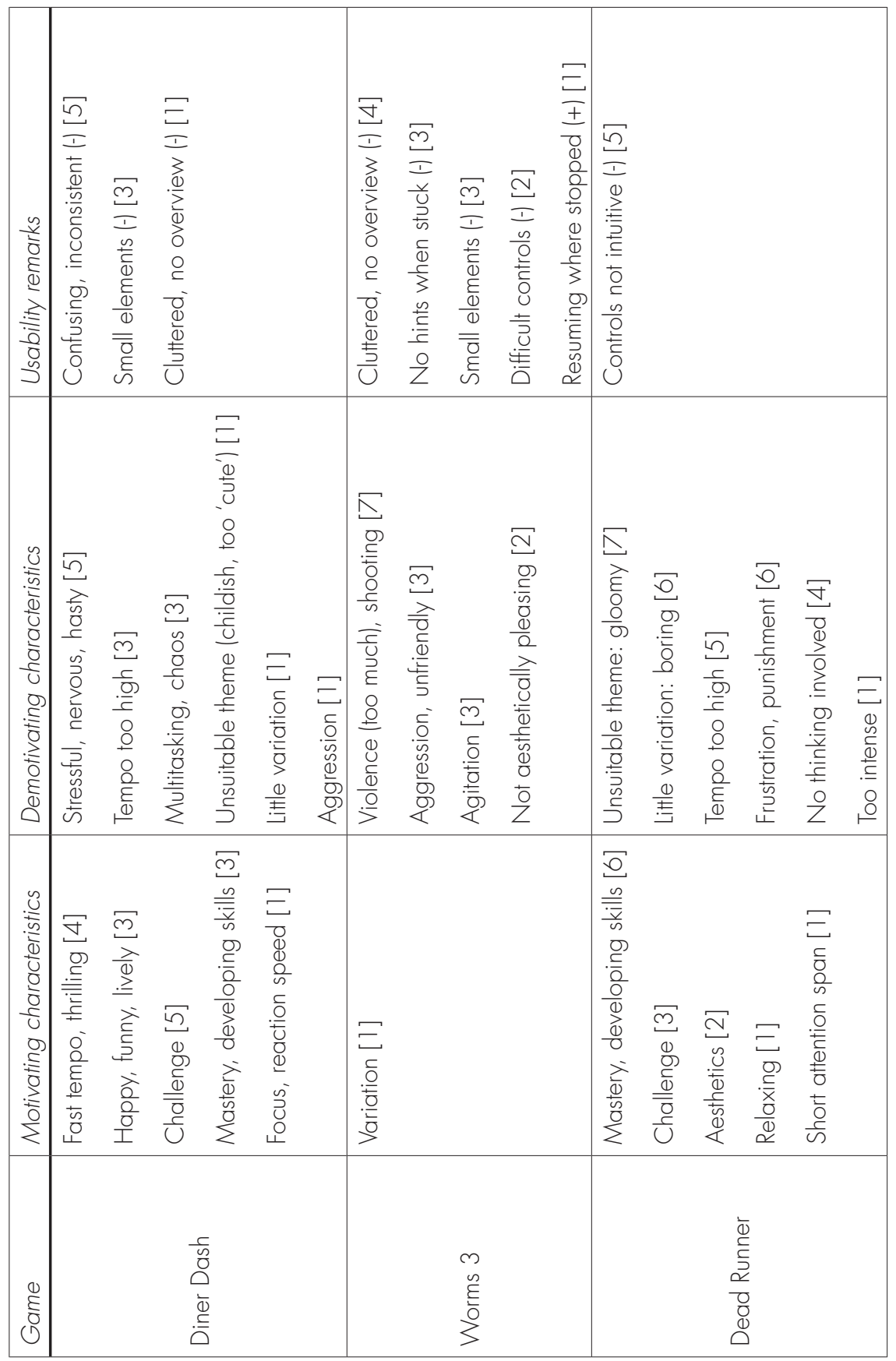




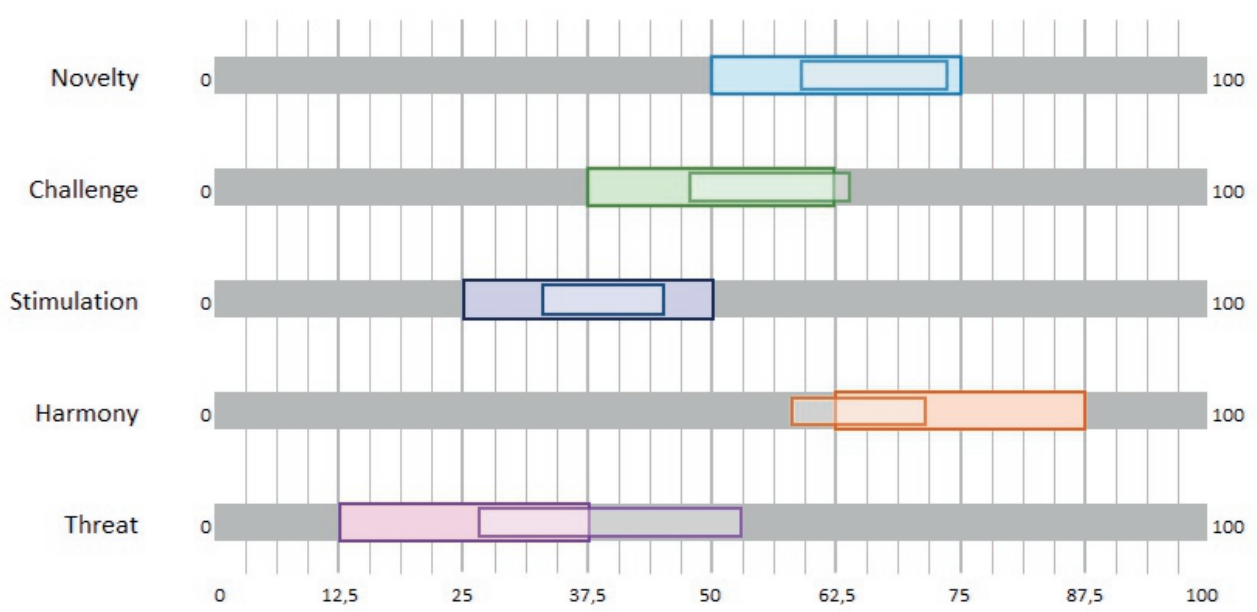

Figure 4 - The profile of Monument Valley (taller bars) against the profile of the older adult (shorter, filled bars)

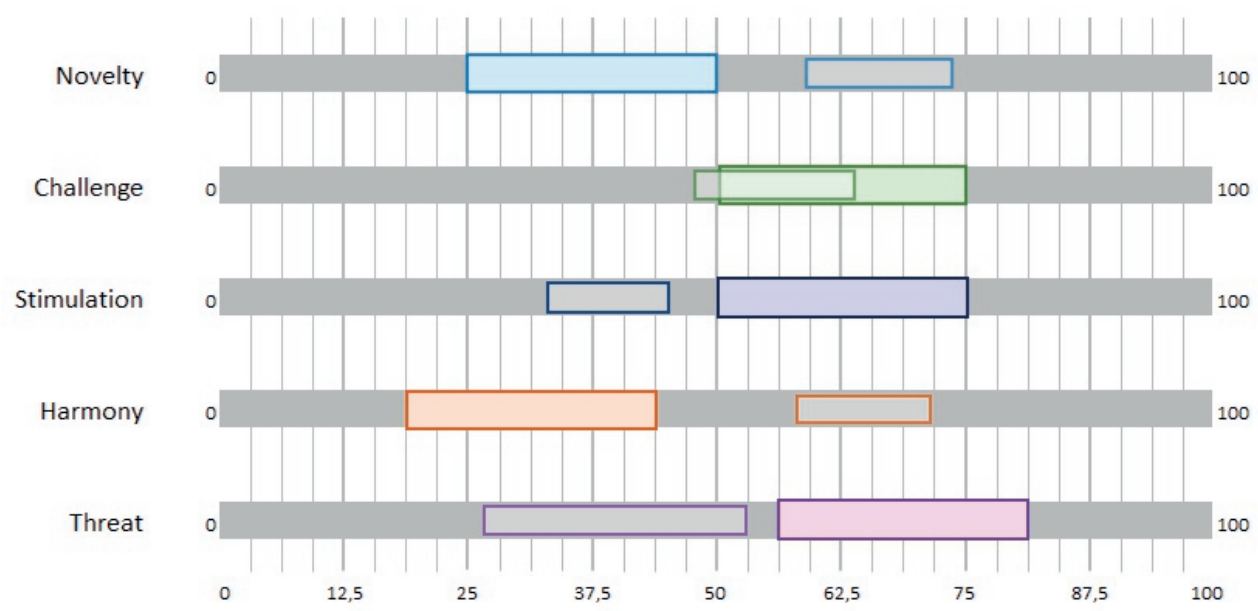

Figure 5 - The profile of Worms (taller bars) against the profile of the older adult (shorter, filled bars)

We can visualise this (mis)match by translating our estimated profile for both games, supported by the opinions of experts in the validation session, into two graphs (see fig. 4, fig. 5). For Monument Valley the higher Novelty, average Challenge, lower Stimulation, high Harmony and low Threat (filled bars) are closely related to the domain scores from the questionnaire (smaller, transparent bars). For Worms, the low Novelty, higher Challenge and Stimulation, low Harmony and high Threat seem very inappropriate for the target group. In an optimal setting we would have validated these graphs in the expert meeting beforehand. 
In addition to the profile of the preferences of the group of older adult users that participated, based on the characteristics as tested in this study, we recommend the following for the selection of games or designing new content for this target group:

- Novelty The target group is open to modern games and new experiences, values aesthetics and plenty of variation. Problem solving and intellect are motivating. Restrictions on this domain are that goals must be clear at all times, while discovery is valued.

- Challenge Whenever appropriate for the underlying (healthcare) application, we recommend focusing game design on cognitive challenge rather than physical. The actual degree of challenge cannot be generalised for a group and is difficult to measure objectively, but the target group should not be underestimated by presenting them games that are too superficial and easy. Users enjoy achieving goals, progression and development of skills.

- Stimulation High intensity and speed are found to be demotivating, in concordance with known usability requirements. Many people find it enjoyable to withdraw and relax with a game on their own, so we cannot be conclusive about the necessity of a social component yet.

- Harmony Violence should at all times be absent from games. An overly cute or childish design is often disliked as well.

- Threat A much lower tolerance for frustration and negativism should be taken into account. We recommend avoiding game mechanics that present feelings of unfairness or lack of control.

To apply games in telemedicine context, we observe the following from our data. Firstly, we may be able to decide on a strategy in terms of game content tailored to the individual or to a group of users. We regard the older adult user as a fairly uniform target group. Prominent subgroups that differ themselves through clustered game preferences, such as may have been the case for differences in affinity with games or technology, were not found. More data is of course needed as the cohort was small and the participants all experienced with tablets and digital games. However, we expect to find similar results if we expand the number of participants. We may be able to generalise the older adult's previous experience with computer games as well as their current awareness of and access to modern video games. The amount of variation in preferences per domain is limited as well. This would mean that, on the condition that enough variation is offered to suit all users, a single game concept may be able to engage the whole target group. In this lies the risk that the engagement for each user is moderate and never high, while the almost unanimous preference for the game Monument Valley refutes this. Other factors that may be of influence on engagement in a treatment context that deserve much to be explored are social context and the effect of social contact through or around the game. The personalisation of content and user-generated content may be valuable additions. 


\subsection{Classification weaknesses}

The findings of this study lead to several refinements of the model domains for future work. We believe it is worthwhile to focus on further developing this classification, but we find that two domains in particular do not allow for precise expression of preferences. Firstly the element of competition, being part of (a low score on) the domain Harmony is overshadowed by violence, placed in the same domain extreme. Participants did not recognise the competitive element in Worms, which was clearly present in our view, possibly from disliking the game so much. In our demographics, we find that most players value competition. Similarly, Diner Dash did not present a suitable frame of reference to the participant on the elements solo versus multiplayer or communication. The majority of participants indicated to like conventional (board/card) games for social contact. This may be entirely different for digital games, but we missed the means to measure or express this separately from the intensity of gameplay. Lastly, the domain Challenge is too unspecific as Challenge should always be present in the proper ratio to create satisfying gameplay. This is highly individual, making it difficult to objectively measure how challenging a game should be. Each player valued a game that is challenging, which we think is accomplished by adequately meeting the balance between skills and challenge as would be described by the principle of flow [31]. We found the lack of refinement in the domains to express certain aspects, for example (the appreciation of problem solving and intellect in the domain Novelty. Future work will aim to further refine this model and add more semantics to the domain extremes.

Besides a refinement of the classification itself, we see the need of an extra step in the methods to assess preferences of a target group such as the older adult, whom are less experienced consumers of video games. There is a discrepancy between the view the older and younger population have on the same game content, which may lead to wrong interpretation of the preferences measured by researchers and game developers. In future work, we should therefore seek methods to compensate or make explicit this difference.

\subsection{Limitations}

The sample of participants of this study leads to several limitations when generalising our findings for a greater public. Firstly, an inclusion criterion - to avoid distraction from the rating of game elements by usability issues - was that participants should be able to work with modern technology such as a tablet or smartphone. As such, participants had a high computer literacy level and results may not apply to older adults that have not. Secondly, albeit a repetition, users of this age that are not intrinsically motivated by games already may not be motivated by a game-based application at all. The ratio between men and women and frequent or non-frequent players is well-balanced, and does not seem to influence preferences scores, and evident sub-groups are absent. Most importantly, while the sample size of this study was sufficient for exploratory research we should keep in mind that another sample 
may lead to a much different profile, and that we therefore cannot extrapolate our findings to the older adult in general. Moreover, few groups are so varied and so rapidly changing as the older generation, where a difference in age nonrepresentative for the physical and cognitive health of people, neither on their skills, openness to and previous experience with games and technology. In a larger cohort, group distribution may need to be taken into account as differentiation between subgroups may be necessary. Lastly, given the small cohort, we chose a number of five games to avoid overload of participants. A follow-up with another selection of five games for each opposite end of each domain would provide a valuable comparison to the results. Furthermore, in order to give more meaning to the time played with each game, results would benefit from a comparison of time played by younger adults.

\section{6| Conclusion}

In search for methods to tailor game design to the preferences of the older adult, thereby effectively engaging them in the long-term use of telemedicine applications, we have investigated and captured the preferences of the older adult regarding game content. These preferences resulted in recommendations for future game design, such as to include novelty over conventional content and to focus on cognitive challenge. This study has led to a better insight in how to address this target group through games and provided a promising starting point towards a method for surveying and mapping preferences of users. Future work will aim to further refine the model and evaluate our findings by putting the method to practice. 


\section{How to design game-}

based healthcare applications for children? A study on children's game preferences

In: Proceedings of the 11th International Joint Conference on Biomedical Engineering Systems and Technologies, HealthiNF 2018. 


\section{Abstract}

Game-based design can be used to develop engaging health applications for children. This engagement can only be realised when design is tailored to their preferences. In this study we investigate game preferences of children and translate these into design recommendations. Game preferences of children aged 6 to 12 were assessed through a questionnaire. Outcomes were classified by means of the 7D framework which divides game content into seven linear domains. Significant differences in mean scores among demographic subgroups were explored. Sixtyfive children participated $(M=9$ years, $S D=0.24,36$ boys, 29 girls, 8 children with asthma). Data showed high preference for content in domains novelty $\left(M_{\text {novely }}\right.$ = 63) and dedication $\left(M_{\text {dedication }}=70\right)$. Analysis resulted in subdivision of scores based on gender, age and playing frequency. Striking differences in scores were found between boys and girls in discord $\left(M_{\text {boys }}=62, M_{\text {girls }}=19\right)$, intensity $\left(M_{\text {boys }}\right.$ $\left.=60, M_{\text {girls }}=27\right)$, rivalry $\left(M_{\text {boys }}=53, M_{\text {girls }}=31\right)$ and threat $\left(M_{\text {boys }}=64, M_{\text {girls }}=\right.$ 25). To design games for children we recommend to stimulate curiosity by offering variation and discovery, to enable achievement, learning and social contact. A divergence in preferences for boys and girls must be regarded. Opposed to boys, girls may lose interest in games that have violent or scary content, that are mainly competitive or demand continuous effort.

\section{1| Introduction}

Health informatics can bridge the distance between healthcare professionals and enable patients to receive treatment in their daily living environment [1], thereby alleviating the increasing demand for care and improving the autonomy and quality of life of patients. Despite that such telemedicine applications contribute to positive health outcomes [2], adherence seems restricted to several weeks of use $[3,4]$. Game-based design may be a strategy to increase adherence as introducing elements of game content to telemedicine applications is hypothesised to better engage the patient in using the application [5], thereby facilitating underlying treatment objectives [6]. Moreover, applying game-based design may be able to sustain engagement for a prolonged time.

In order to successfully design game content that appeals to a specific target group, an understanding of their preferences regarding this game content is essential. To express these preferences, we can describe the user in various ways. Two commonly used approaches are player taxonomies and game genre (or form) classifications. A well-known player taxonomy is the Bartle player typology [7], which classifies players into four types that represent the player's preferred behaviour within the game. Such player taxonomies $[7,8]$ usually originate from analysis of a specific type of game and its players, and can therefore not be generalised or used outside 
Characteristics

$$
\begin{array}{r}
\text { Fairness } \\
\text { Compassion }
\end{array}
$$

Serenity

Teamplay

Altruism

Habitual
Simplicity

Low self-discipline

Familiarity
Routine
Expectedness

Low social engagement Non-communicative

No time pressure Short attention span

Low speed

Safety
Comforting
Pleasant
Domain

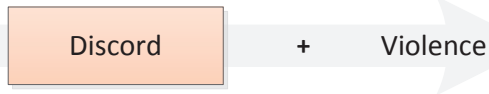

+ Competition

Cooperation

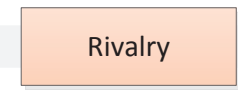

Casual

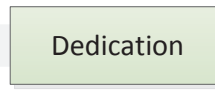

$+\quad$ Devoted

Conventional

Solitary

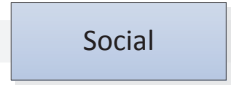

+ Multi-player

Slow-paced
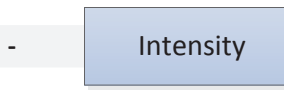

Threat

Calm
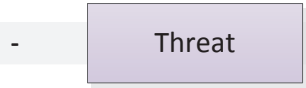

\section{Characteristics}

Cruelty

Destruction

Intimidation

Gaining, winning

Defeating

Strife

Mastery

Achievement

Commitment

Curiosity

Surprise

Fantasy

Company, social interaction Communicative

Fast

Intense

Thrilling

Frustration

Gloom

Tension

Figure 1: The 7D framework - a model for game content along seven linear domains.

of their context [9]. Also, the classes the models consist of are fixed and non-linear, thereby not forming a complete description of all preferences a user may have. The other approach, expressing preferences through game genre or form (such as a simulation, shooter, educational, role-playing, etc.), gives an overall representation of a set of game features, which is not uniformly described among various game developers [10]. Neither of these approaches therefore allow for accurate assessment of preferences that is needed to synthesise into recommendations for the design of new game content, as the information on the player from a player typology is limited and too high level from a genre classification.

Instead, particularly as we envision to add game content to healthcare applications to prolong adherence, information on the player's preferences needs to be more detailed. This would correspond to a model that allows us to describe games by their actual characteristics, which would enable us to express preference of the user for specific game content on a much more profound level. We expect that by using such a model, we have more insight in how the individual should be addressed through game design but also how a group of users should be approached to realise more engagement. 
As such, we developed a model for the classification of game content in earlier work (De Vette et al., 2017); the '7D framework'. This model can be used to assess and map the preferences of users for game content, resulting in a detailed description of these preferences that can be used to design new content. The model can be used to gain insight in whether or not demographic or psychosocial differences exist that may have implications on the design of the game content should be taken into account. The 7D framework can also be used to analyse and make explicit the content of existing games. The theoretical foundations for this model originate from the five factor model of personality [ 11 ] and its translation into gaming semantics, the five domains of play theory [12]. The 7D framework structures game content along seven linear domains that are defined by a set of characteristics per each domain extreme, as shown in figure 1. Discord and rivalry were originally represented by a single domain in previous versions of the model, as were social and intensity, hence they have been given the same colour in the figure.

Applying the 7D framework and its precursors to investigate the preferences of older adults group showed, in short, their preference for high novelty content and (cognitive) challenges, and an outspoken disfavour for intense, violent and frustrating content $[13,14]$. This research resulted in the development of a game-based selfmanagement platform for older adults [15] using a storyline, enabling exploration, containing puzzle-oriented mini-games to sustain the motivation of the user. This game is currently under evaluation to study its effectiveness, but from preliminary results we find that the translation of the user preferences into game design has been successful. Now, we aim to explore the use of this model to gain insight in the preferences of other groups.

Unlike the older adult, children are a more common consumer of video games making the success of a game-based approach in healthcare applications more likely. Games are regularly applied for children outside the goal of entertainment, and numerous examples of serious games [16] and game-based learning programs can be found [17]. However, additional research is needed to determine the game design that best promotes effectiveness of games in health informatics [18]. Just like older adults, children do not fit within the researched target groups using existing user classifications, nor do these models fit the context of digital healthcare applications. To the best of our knowledge, we are unaware of an existing framework that can be used to measure and elaborate game preferences of children on the level of core game content.

In this study we aim to gain insight in the preferences of children regarding game content and translate these preferences into design recommendations for engaging game content for children. To do so, we assess the preferences of children along the 7D framework and research whether significant differences in preferences exist among subgroups of children. This could give us a starting position in tailoring game-based telemedicine applications to this target group. 

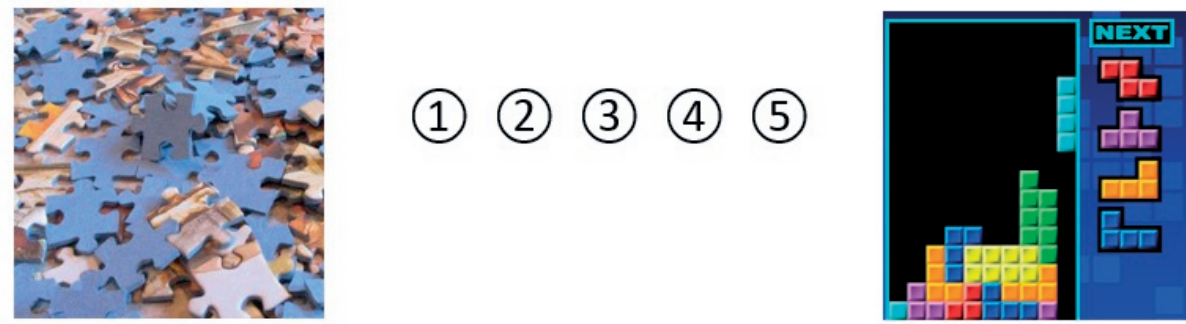

\section{$k$. lets vrolijks}

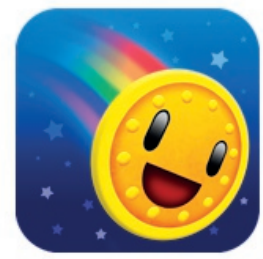

(1) (2) (3) (4) (5)

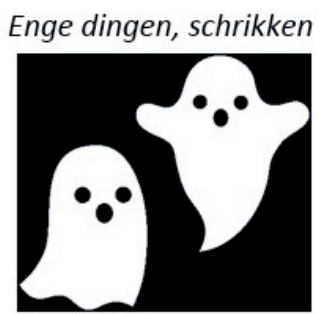

Figure 2: Fragment from the questionnaire (questions measuring intensity and threat)

\section{2| Methods}

\subsection{Design and participants}

The study is cross-sectional. The participants were school-going children in the age of 6 to 12 years old. Local primary schools were invited for a Science Day taking place at University of Twente, the Netherlands. During this day, children were taught about the university and invited to participate in all kinds of activities related to technological innovation. Participants and their parents were informed on beforehand about the activities of the Science Day by the organisation of the event through an information letter. In addition, a second group of children with asthma within the same age range participated one day later. These participants were recruited via the children's department at Medisch Spectrum Twente, Enschede. For both groups, parents gave their informed consent prior to participation. Also, children's participation was voluntary and no exclusion criteria were applied.

One of the activities was playing on an interactive playground developed in the AIRPlay project [20], to support self-management of children with asthma and to promote their physical activity in a fun and unobtrusive manner. This playground uses floor projection and movement tracking to generate the playing field for a game inspired by the children's game 'tag'. 


\subsection{Measures and materials}

We assessed the preferences of children by using a questionnaire based on the $7 D$ framework. The domains, as shown in figure 1, are 1) discord, which features peaceful versus violent content, 2) rivalry features cooperation versus competition, 3) dedication the appreciation of a game that requires low self-discipline ('casual') versus a game that is achievement-oriented ('devoted'), 4) novelty the preference for conventional, routinely or real-world versus fantasy, curiosity and variation, 5) social features the opposition of the amount of interaction with others, between solitary and multiplayer, 6) intensity holds slow and relaxed against fast, intense and time pressured, 7) threat the acceptable amount of negative feelings that the game can cause in the player, ranging from calm and lovely to frustration and fear.

Each domain is a linear scale (ranging from 0 to 100) described by two extremes. These extremes are described by characteristics, that can be translated into actual game features. We deduce our recommendations for game design from the measured scores on the different domains, and on our analysis of scores of identified subgroups.

The questionnaire was adapted to the target group by keeping the questions concise and illustrating them with simple images. Also, the number of questions was limited to a minimum of 11 questions by measuring two aspects of the more complex domains of rivalry, dedication, novelty and social, and one of the simpler domains intensity, threat and discord. All questions were rated on a 1 to 5 scale, each question was illustrated by two images of the extremes and an example in text (fig. 2). The question was 'Which [example] do you like more in a game?' ("I like [example A] better than [example B]"). The average scores were calculated for the seven domains and expressed in terms of percentages.

Demographics, including information on favourite games, playing behaviour and access to devices, were assessed in the questionnaire.

\subsection{Procedures}

Measurements of both groups took place at University of Twente and occurred in the same manner. Groups of up to eight children were invited to play on the interactive playground. The children took turns in forming two teams of two players. Playing continued for approximately five minutes. Subsequently, the group of 8 participants was asked to fill in the questionnaire.

\subsection{Data analysis}

Data analysis was performed using Statistical Package for Social Sciences (SPSS v.22). Data distributions of the domains were analysed using the Shapiro-Wilk test, descriptives and boxplots. All domains were found non-normally distributed for all participants. Results are presented as mean and median domain scores. Subgroups 
Table 1: Average scores on the seven domains (significant differences $(\boldsymbol{\alpha}<0,05)$ in means highlighted).

\begin{tabular}{|c|c|c|c|c|c|c|c|c|}
\hline & & Discord & Rivalry & Dedication & Novelty & Social & Intensity & Threat \\
\hline \multirow[t]{2}{*}{ All $(n=65)$} & mean & 43 & 43 & 70 & 63 & 58 & 45 & 47 \\
\hline & median & 38 & 50 & 75 & 63 & 50 & 50 & 50 \\
\hline \multirow[t]{2}{*}{ Boys $(n=36)$} & mean & 62 & 53 & 74 & 60 & 51 & 60 & 64 \\
\hline & median & 63 & 50 & 100 & 63 & 50 & 75 & 75 \\
\hline \multirow[t]{2}{*}{ Girls $(n=29)$} & mean & 19 & 31 & 65 & 66 & 66 & 27 & 25 \\
\hline & median & 13 & 25 & 75 & 75 & 75 & 25 & 13 \\
\hline \multirow[t]{2}{*}{$6-9$ years $(n=28)$} & mean & 47 & 47 & 67 & 64 & 50 & 42 & 49 \\
\hline & median & 44 & 50 & 100 & 69 & 50 & 50 & 50 \\
\hline \multirow[t]{2}{*}{$10-12$ years $(n=31)$} & mean & 38 & 39 & 73 & 61 & 66 & 49 & 44 \\
\hline & median & 38 & 50 & 75 & 63 & 75 & 50 & 50 \\
\hline \multirow[t]{2}{*}{ Frequent players ( $n=29$ ) } & mean & 58 & 53 & 76 & 66 & 53 & 67 & 66 \\
\hline & median & 63 & 50 & 100 & 63 & 50 & 75 & 75 \\
\hline \multirow[t]{2}{*}{ Non-frequent players $(n=28)$} & mean & 25 & 34 & 64 & 64 & 64 & 26 & 30 \\
\hline & median & 25 & 25 & 75 & 69 & 63 & 25 & 13 \\
\hline \multirow[t]{2}{*}{ Asthma (n = 8) } & mean & 48 & 41 & 69 & 47 & 55 & 34 & 34 \\
\hline & median & 63 & 25 & 63 & 50 & 50 & 0 & 25 \\
\hline \multirow[t]{2}{*}{ Non-asthma $(\mathrm{n}=57)$} & mean & 42 & 43 & 70 & 65 & 58 & 47 & 48 \\
\hline & median & 38 & 50 & 75 & 63 & 50 & 50 & 50 \\
\hline
\end{tabular}

were identified by comparing mean scores and analysing significance levels using the Mann-Whitney test for nonparametric distributions.

The principal findings are presented in a visual overview using the domain scores of all participant as well as the most striking subgroup(s) based on the highest number of significantly different domain score means. Univariate linear regression analysis was used to study the explained variance in the domains by both gender and playing frequency.

\section{3| Results}

Sixty-five children participated (mean age 9, 36 boys and 29 girls). Table 1 shows the game preferences of all participants. Subgroups were identified in comparing means in gender, age and playing frequency. This resulted in partitioning of gender in 1) boys and 2) girls, age in 1) 6 to 9 years old and 2) 10 to 12 years old, and playing frequency in 1) frequent players (daily to at least weekly) and 2) non-frequent players (not regular to never at all). A trending difference $(p<0,10)$ was found between the scores of children with and without asthma on the domain novelty $(\mathrm{M}$ asthma $=47, \mathrm{M}$ non-asthma $=65)$. 
Figure 3 visualises the scores of all participants and of boys and girls, as this subgroup shows the largest number of significant differences. This graph shows the uniformity in scores on dedication, novelty and social, and the scores on discord, rivalry, intensity and threat that appear to be determined by the clear division in scores of boys and girls.

The domains dedication and novelty receive the highest scores from all participants, indicating an outspoken preference for content in these domains. The score on dedication suggests that these children have a preference for games that require effort and in which goals can be achieved. The score on novelty suggests a preference for variation, discovery, fantasy and creativity instead of more predictable content, such as football or racing. Significant differences were found between boys and girls on the domains discord, rivalry, social, intensity and threat. Social involvement through a game seems to be preferred by girls more than boys. On average, girls indicate a much different preference for discord, rivalry, intensity and threat. On the scale of discord, in which participants indicate their preferences between peaceful and violent content, boys give a high and girls a low score. A contrast of the same extent can be found in intensity, that separates a relaxed activity from one demanding focus and attention, and the presence of time pressure and speed, and threat, cheerful against disturbing content. Also in rivalry, in which preference between working together and competing as well as helping others to win against defeating others is measured, boys indicated a much higher preference for the latter.

A significant difference was found between the means of the two age groups on the domain social $(p=0,033)$. Upon analysis of the histograms of the two age groups (data not shown) we find that the younger group is more inclined to choose for the extremes (values 1, 3 or 5) while the older group is capable of indicating preferences on a more subtle level (also using values 2 and 4). All except one participant, who preferred playing alone, indicated to prefer playing together with classmates, friends in the neighbourhood or sports club and/or siblings or other family members.

Significant differences in scores for different playing frequencies were found in domains discord, rivalry, intensity and threat (missing data for 8 children who did not answer this question). Frequent players score on average about twice as high on these domains than the less frequent players. $81 \%$ of boys indicates to be a frequent player, of the girls this is $15 \%$. However, there is a high significant correlation between gender and playing frequency. Gender shows to be a more important predictor for the domain scores discord rivalry and threat than playing frequency based on higher explained variance in linear regression analyses, while playing frequency was more important for the domain intensity (data not shown). Subanalysis of the scores of frequently playing boys $(n=25)$ and non-frequently playing boys $(n=11)$ shows that their preferences differ on the domain intensity ( $M$ frequent $=72, \mathrm{M}$ non-frequent $=34$ ). 


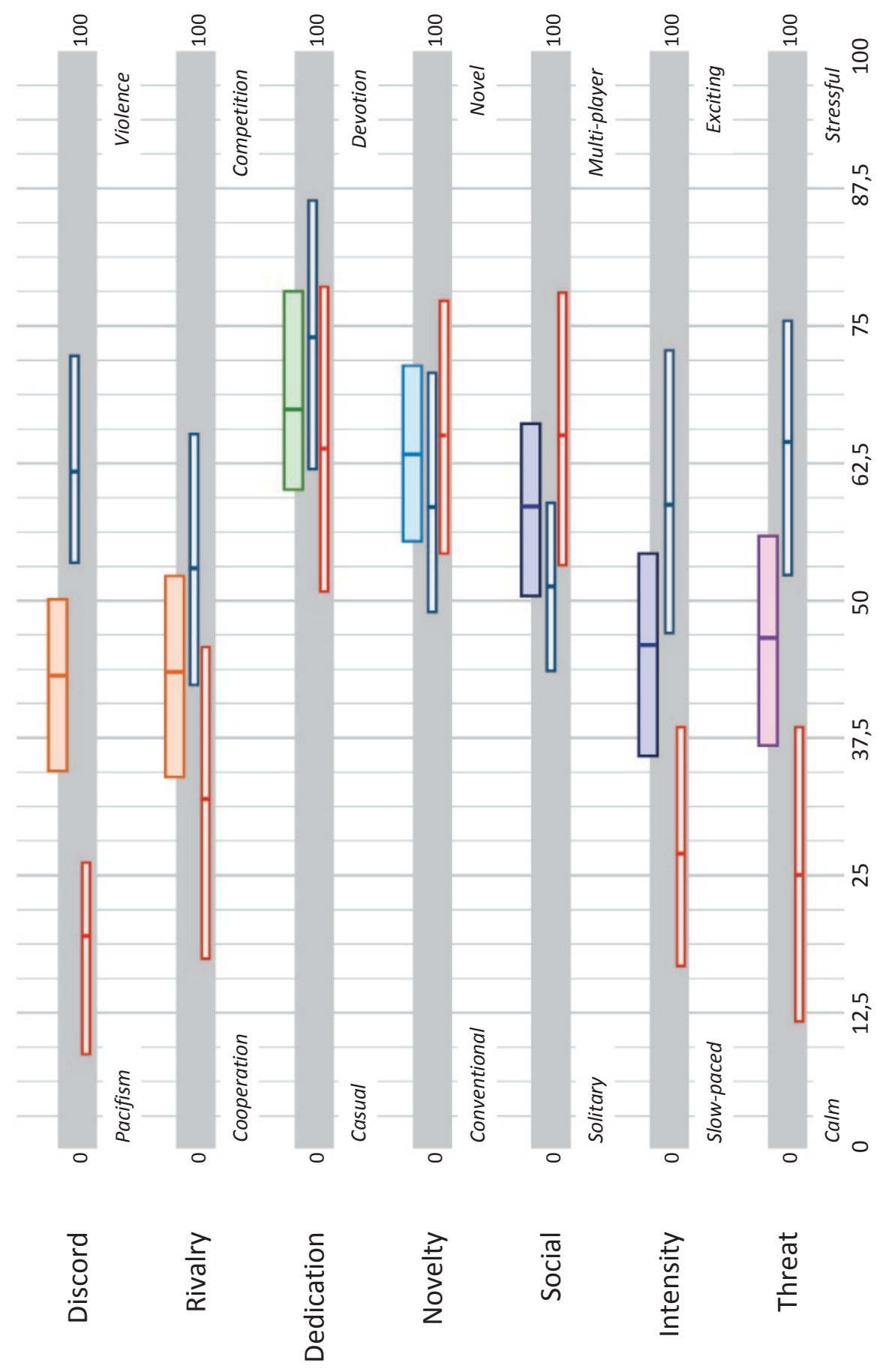

Figure 3: Overview of preferences mapped on the 7D framework (average and 95\% interval domain scores) of all participants (top), boys (middle, blue) and girls (lower, red). The upper box in each domain shows the average score of all users and the 95\% interval. The smaller boxes show the scores of boys (middle) and girls (lower) in the same manner. 
Looking into favourite devices and games of both groups we see that children in the high playing frequency group indicates to favour using consoles such as PS4 to play games such as Call of Duty and GTA, and children in the low playing frequency group often do not indicate a specific favourite or mention a website offering various mini-games. From all participants, 14 children indicate a specific console or PC game as their favourite game, 15 children mention Minecraft (5 of them girls).

\section{4| Recommendations}

Based on the findings of this study we recommend the following when aiming to engage children through game design. These recommendations suit the approach of designing one game to address the full target group.

Novelty: focus on stimulating the children's curiosity and avoid routine. New game content should be introduced regularly. Variation can be created through for example new rules, mechanisms or visual elements. Enabling children to use their imagination, be creative and add their own variations to the content, or enable emergent gameplay, may be a valuable feature. A novelty theme (fantastical, fictional) may also be more suitable than a real-world theme.

Dedication: provide content that enables achievement. Games should always be sufficiently challenging. For children, it may demand an effort to learn skills necessary to play the game. A trial-and-error approach to do so can be rewarding. Include clear feedback on achievements. Making content unlockable may serve both the preferences on novelty and dedication.

Social: a game should enable playing together. The preference for social contact is slightly dependent on the age and gender ratio of the group, but in general children prefer playing together rather than on their own.

Discord and threat: a neutral approach would be advised on violence and scary content. We observed a preference for violent video games particularly in boys. Most girls may be put off quickly by for example fighting games, as most boys will probably not be interested in overly cute games. Violent and scary game content should always be age appropriate.

Rivalry: competition and cooperation may be used alternately to keep a game interesting for both boys and girls. In a multiplayer game, team play or 'helper' functions may be added next to mechanics enabling competition, such as setting challenges for other players. 
Intensity: a moderate intensity should address both boys and girls. We do however recommend keeping in mind the preference for lower intensity of most girls. Games should provide the opportunity to choose an intense as well as a more laid-back playing style to avoid girls losing interest from a game that demands continuous effort, movement or focus. We believe that this is the case particularly when developing games that involve physical exercise.

\section{5| Discussion}

The aim of this study was to gain insight in the game preferences of children, in order to synthesise recommendations for game design to support the development of engaging game-based telemedicine applications. Using the 7D framework, the preferences of 65 children were assessed resulting in a graphical overview of preferences. The scores suggest an overall approach towards game design for this target group when attempting to engage all members of the target group through one game, which has been the basis for a set of game design recommendations. It is important to identify and take into account different preferences from subgroups within a population to avoid that game content is developed that may either be too much of a compromise or is disliked by the majority of one of both groups. While gender was a more important predictor to game preference in this study, we do not assume that we should ignore playing frequency. We know that girls are interested in games but that they prefer much different characteristics [21]. The current offer of videogames may determine that mostly boys are the frequent players. There is a group of boys that play frequently who indicated a much higher preference for violent, intense and disturbing content than the boys that play less often. Also, some have access to consoles with games that may be considered inappropriate for their age because of their violent content. We expect that this subgroup may be hard to please, if at all, as they are used to high-end games, which moreover serve a set of preferences that may put off many others.

Children with chronic conditions may form another subgroup. Carefully interpreting the scores, we expect that these children are more reserved (lower novelty) and choose for a game that is less intense (lower intensity). Recommendations for game design for this group may include for example giving them the chance to discretely take breaks or exhaust themselves less than the others. More data is needed to better understand the impact a chronic condition, such as asthma, may have on game design.

Several aspects of this study lead to limitations when generalising our findings for a greater public. Firstly, our sample included children from local primary schools. Cultural and educational differences that may be of great influence on the results were not taken into account. Divergent reading and writing abilities among participants, 
not necessarily related to age, may have led to misinterpretation of the questions. Also, the Science Day and the playing on the AIRPlay interactive playground may have influenced the results. Secondly, validation of the 7D framework is currently in progress. As such, the creation of the questionnaire as well as the interpretation scores into recommendations is to some extent subjective. It is vital that the intention of the designer is in accordance with the perception of the target user on game content, future work should include measures to align both frames of reference. For example, a game designer may develop gameplay that seems very intense for a child, while the child itself interprets this differently. Furthermore, it should be noted that the interpretation of the recommendations given is open to the game designer, as creating a satisfying game is not merely a sum of parts but a design process in itself. As an example, a high score on dedication indicates that it is more likely that the user prefers game content that demands devotion to play. This is described by the example characteristics mastery, achievement and commitment. In case of a high score on dedication, we would recommend to include the need for skill development in order to advance in the game (unlike a more habitual game that would always require the same amount of skill). A game designer may then choose how to realise this aspect. Lastly, this study included a limited number of participants with asthma. We expect that minor differences will show between the asthma and non-asthma group in a larger dataset. At the time of writing the dataset is being expanded with the data of more children with asthma.

In current and future work, we aim to fill in a research gap on the existence of a method to elaborate the preferences of any target group into a specific characterisation to provide a starting position for game-based design in health informatics. To ensure validity of the 7D framework, we would propose to extend research with 1) a comparison of the results with existing literature on children's playing behaviour, preferences and personality, 2) creating a more elaborate questionnaire that includes a larger number of game content characteristics, as we assume this can lead to more detailed recommendations for game design, and to 3) apply the design recommendations in practice to evaluate to measure their effectiveness in engaging the target group to the healthcare application. Furthermore, we aim to investigate if certain domains are more important than others to the overall engagement when developing a strategy for game design. In future work we aim to respond to any differences that may be found in preferences for children with or without chronic conditions, in order to predict which strategies may be successful for these children based on game preferences, besides attitude or different physical capabilities. 

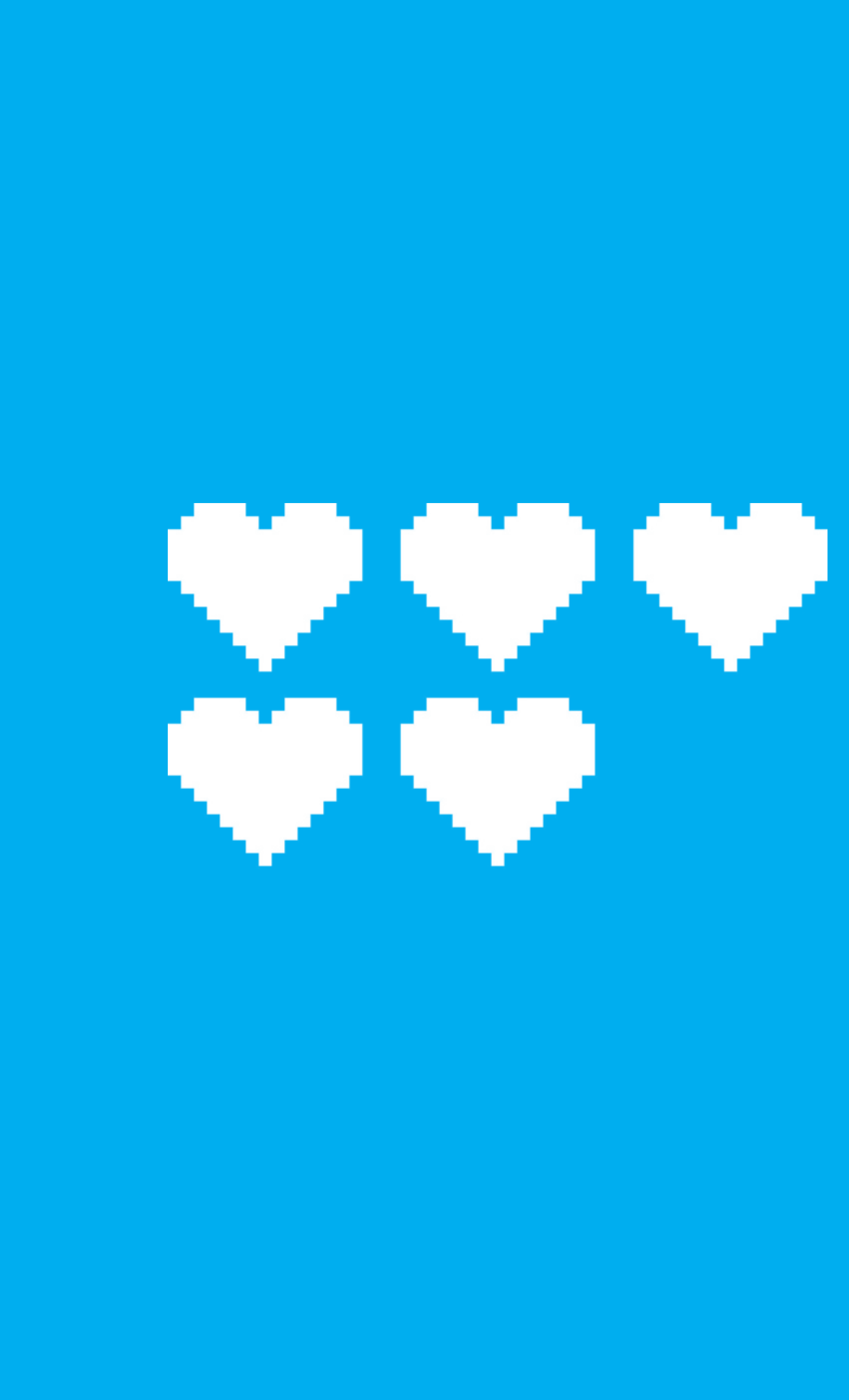

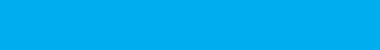
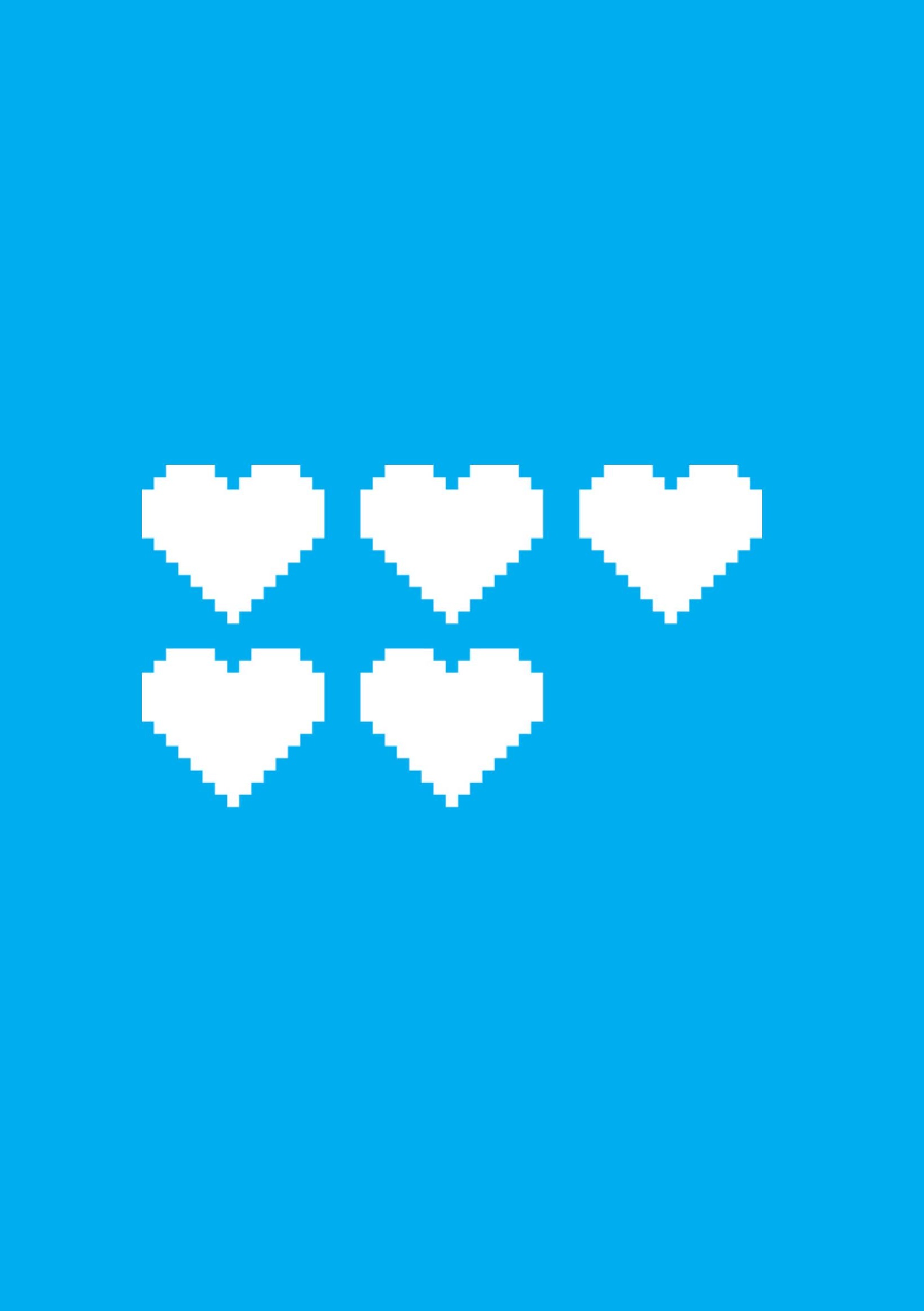


\section{Abstract}

In an effort to improve engagement to 'serious' game-based applications, we aim to create a framework that enables game designers to identify their target audience's key motivations to play. We developed the 6D framework: a classification for game content and a survey to assess user preferences for this content. In this study, we 1) refined and validated the framework and 2) demonstrated the framework in practice. Three rounds of expert panel sessions resulted in a revised version of the framework's classification and survey, of which content validity was checked. In an online study, 102 respondents filled in the framework's survey resulting in profiles of their game preferences as well as a practical example of insights gained from applying the framework. We found an average $76 \%$ match in comparing the profiles of the user with the user's top ranked games. This study resulted in a handson method for game-based application developers.

\section{1| Introduction}

The growing popularity of games has induced a trend in the use of games as a motivational strategy outside its original context of entertainment. More and more full games (e.g. serious games, exergames) or a selection of elements from games implemented in an application (gamification, gameful design) to support engagement in fields such as healthcare [1,2] and education [3-5]. We aim to improve this strategy by responding to the game preferences of the target user in designing new game-based applications [6], and aid designers of such applications by developing an evidence-based framework [7] and avoiding a one-size-fits-all approach [8].

A suitable framework to identify these preferences providing concrete leads on suitable game content, consisting of both a scientifically grounded theoretical model and a practical method for assessment, was not found among current practice [9]. As such, we aim to contribute to this gap in research by developing a new evidence-based framework to aid designers of various game-based applications through gaining insight in their target group.

The framework enables us to create a broad overview of the high-level game content that contributes to a satisfying gaming experience, therefore engages in play. We started by setting theoretical foundation of the framework through a new classification for game content. Next, we developed a survey to assess the preferences of the end users for this content and map these onto the classification. From several player type taxonomies we explored in earlier work [9], such as the models by Yee [10] and LeBlanc [11], we chose the Five Domains of Play theory [12] as a point of departure for this new classification for game content. 
The framework was refined through application in practice in a series of five studies ( $n=365$ ) with diverse target groups (adults, older adults and children), for which we refer to earlier articles [13-15]. These studies resulted in the framework's classification to be more accurate in expressing game content, and the design of the survey most suitable for a particular target group.

The aim of this study was to finalise the framework and convert it into a handson method to investigate the target audience's preferences for game content, that includes practical leads for designing engaging game content. We thereby aimed for an optimal trade-off in effort and resulting insight in game preferences. The study consists of two steps: 1) refinement and validation and 2) a practical demonstration study in reallife setting, to show the insight that is gained from applying the framework in practice.

\section{Related work}

Current examples of game-based design in this field have been dominated by industry publications and frameworks [16]. While attractive for their success in business context, practical applicability and simplicity, the majority of these frameworks are not grounded in scientific research [17] nor suitable for non-entertainment contexts $[18,19]$. The popular industry practice in gamification centers around extrinsic motivation through positive game experience, as for example 'smart gamification' by Kim [20], 'six steps to gamification' by Werbach and Hunter [21], 'gamification by design' by Zicherman and Cunningham [22], 'the gamification of learning and instruction' by Kapp [23] and 'gamify' by Burke [24]. Many methods are criticised for using unsuitable stereotypes (Bartle [25] and Schell [26]) or taking on the approach to applying a limited set of the same game elements (Robertson [27], Bogost [28]).

Existing frameworks that are rooted in scientific research with similar rationale, such as the gamification process by Aparicio et al. [29], the theoretical framework for meaningful gamification by Nicholson [30], the social gamification framework by Simões et al. [31], the value-based design for gamifying daily activities by Sakamoto et al. [32] and designing gamified solutions by Gears and Braun [33], do not provide practical methods for assessment and further application.

Other common practice to express preferences of users in videogames are player type taxonomies, as mentioned earlier in the introduction, or genre classifications. Often, these cannot be generally applied to all target groups and contexts [34]. They are usually focused on a specific audience and vary between game developers [35], are too underdefined or contain multiple information dimensions [36], which all make translation of measured preferences into new game design difficult [37]. Moreover, these methods are particularly unsuitable for those less acquainted with games [14]. 


\section{2| Refinement and validation}

\subsection{Methods}

In the first section of this study, we evaluated the framework's classification for game content and the survey to investigate the preferences for this game content of target users. In three iterative rounds of sessions with a panel of experts, we 1) refined the classification in terms of descriptive semantics of the game content, 2) revised the survey to a concise version and 3) evaluated the content validity of the survey.

\subsubsection{Participants}

The panel consisted of 9 experts 18 male, 1 female, and age 26 to $49, M=$ 33) with a transcendent knowledge of videogames. Experts included performed research in related fields (game design, game studies, persuasive gaming), were involved in (commercial) game development and/or core and hardcore gamers actively involved in gaming community (as per Adams [38]). The main inclusion criterion thereby was having a long-erm experience and interest in games of a preferably wide variety, and/or game research or related fields.

\subsubsection{Materials}

The main material for this study was the most recent version of the framework developed. In the framework's classification, all high-level characteristics of games (which may contribute to a satisfying gaming experience) are categorised by means of a number of 'domains' (see also: results section).

Second, a list of insights gained from application of the framework in practice in previous studies was used. Several discrepancies in the classification were identified, as mentioned in the discussion of previous works. For example, cooperative and competitive gameplay were formerly placed as opposites in the same domain, limiting people to indicate a preference for competitive team play. Also, we observed that the survey we created for our previous studies, containing 45 items addressing most of the classification's characteristics, unnecessarily burdened our target users. In comparison, a simplified 11 -item survey addressing one or two main characteristics per domain, as applied for children, resulted data sufficiently insightful for our application development goals as well. We therefore created a draft version of a new, concise survey beforehand of the expert sessions.

In addition, we used a list of 65 modern game titles, which was compiled beforehand of the sessions as well. This list firstly served in the expert sessions as test cases in analysing their content, in order to reveal any shortcomings and discrepancies in the classification. These games were selected through the libraries of Steam, XBOX (Microsoft) and PlayStation (Sony). We chose to include recent, mainstream popular titles that are not exclusive to specific systems or consoles (such as most Nintendo games). 
Finally, a questionnaire that was created in order to assess the content validity of the framework survey was used. Experts rated the importance of the question to the construct being measured (essential, useful but not essential, not necessary). The questionnaire measured the capability of the survey items to 1) sufficiently represent the game content present in the classification, 2) adequately assess the (preference for) game content present in the classification and 3) cover all classification domains equally respective to each other (all answered by yes/no, should be 'yes' in at least $90 \%$ of cases to suffice). In the questionnaire, the CVR (content validity ratio) was assessed for all items [39]. A positive CVR (should be > 0.78 [40]) suffices for the accurate measurement of user preferences for game content, as well as accurate compilation of content of existing video games.

\subsubsection{Procedures}

In the first round, the aim was to improve the classification in expressing all possible game content that can motivate people to play. The experts were informed in detail on the research background and the framework. The sessions consisted of a discussion on the suitability and accuracy of the semantics used in the classification with up to three members (selected randomly) of the panel. The discussion was structured along the classification domains and led by our findings from previous studies. To aid the discussions, experts were invited to map their own preferences onto the classification and analyse the content of several games. Once all panel member's feedback, revisions and additions to the classification were noted, the classification was directly revised accordingly and recapitulated with all experts. In the second round of sessions, the survey was reviewed. Beforehand, the revisions to the classification resulting from the first round of sessions were incorporated in the survey items. The experts were asked to review the structure and contents of the survey as well as consider its capability to form a complete, representative profile of game preferences. Then, the contents of each game from the list were analysed using the revised framework and a profile (including a general description) was created for each game. To conclude this round, all panel members were individually asked to inspect and approve upon the final game profiles.

In the third and last round, the survey was evaluated by means of the abovementioned content validity questionnaire. This questionnaire was filled in digitally.

\subsection{Results}

The result of the expert evaluation is a new framework, created to assess user motivation in terms of game content: the 6D framework. The classification (figure 1) provides a comprehensive overview of all game content that contributes to a satisfying gaming experience through six classes, which we call 'domains'. Each of these domains is linear and bilateral, meaning they are defined by two 'extremes' that are opposites. These extremes are elaborated by a set of characteristics. The survey enables the assessment of user preferences for this game content, as well 


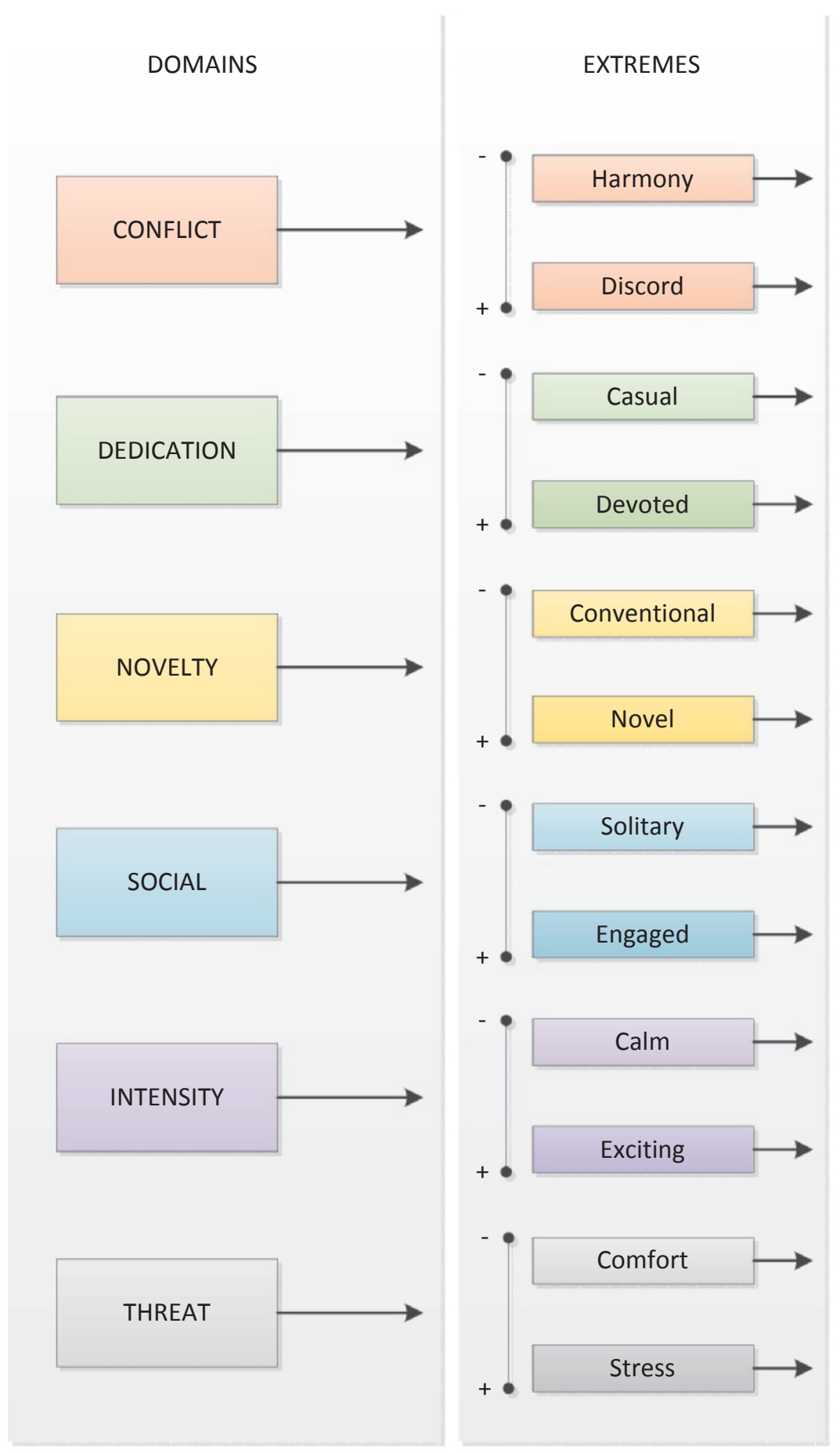

Figure 1: Overview of the 6D framework 


\section{CHARACTERISTICS}

Serenity Pacifism Modesty Compassion Caring

Altruism Friendship Love Attachment Honesty

Fairness Trust Integrity Morality Empathy

Destruction Violence Gore Fighting Aggression Oppression Intimidation

Glory Power Boasting Rivalry Sabotage Trolling Cheating Cruelty Immorality Detachment

\begin{tabular}{|c|}
\hline $\begin{array}{c}\text { Simplicity Impulsiveness Short gameplay Triviality } \\
\text { Nonchalance Cannot die/lose Low effort } \\
\text { No obligations/objectives Short learning curve }\end{array}$ \\
\hline Complexity Commitment Effort Diligence \\
Discipline Competition Achievement-striving \\
Progress Mastery Learning Time investment \\
\hline $\begin{array}{c}\text { Real world Familiarity Concreteness Practicality } \\
\text { Repetition Habitual Expectedness Routine } \\
\text { Continuity Clear boundaries/rules }\end{array}$ \\
\hline
\end{tabular}

Imaginary Fantastical Intellect Abstraction Artistic interest

Culture Storyline Character development Adventure Discovery Surprise Curiosity Choice

Single/solo play Reserved Solitary

Low social engagement Low interaction

Non-communicative Passive Receptive

Team play Cooperation Company Crowds

High social engagement High interaction

Communicative Assertive Expressive

Moderate Slow-paced No time pressure

Low stimulation Low focus Low activity

Laid-back Short attention span

Intense Thrilling Fast Time pressure

Physical Sensory High focus High activity

Action Continuous attention

\section{Relaxing Pleasant Confidence Safety}

Control Comforting Calming Clarity

Cheerful Unrestrained Positive Cuteness

Suspense Frustration Panic Confusion

Vulnerability Humiliation Danger Anxiety Fear

Shock Helplessness Depression Gloom Horror 
as analysis of the content of existing games. Assessment of user preferences, by means of measuring how satisfying the user finds the proposed game content characteristics, results in a user profile. Finally, these preferred characteristics can be translated by a game designer into game features that make up the eventual gameplay. For example, the game characteristic 'achievement-striving' can be expressed by a game features such as missions or a point system.

\begin{tabular}{|c|c|}
\hline \multicolumn{2}{|c|}{$\begin{array}{l}\text { Please rate how satisfying you find the following characteristics in games. You may have many different motiva- } \\
\text { tions to play, so indicate what applies to you most of the time. Use the slider to indicate your preferences. Leave } \\
\text { the slider in the middle when both sides appeal to you equally. "I find it more satisfying when a game is about..." }\end{array}$} \\
\hline Serenity, pacifism, harmony & Destruction, violence, gore, fighting, aggression, discord \\
\hline $\begin{array}{r}\text { Modesty, compassion, altruism, caring, } \\
\text { friendship, love, attachment }\end{array}$ & $\begin{array}{l}\text { Boasting, intimidation, glory, power, } \\
\text { oppression, rivalry, detachment }\end{array}$ \\
\hline Honesty, fairness, trust, integrity, morality, empathy & Sabotage, trolling, cheating, cruelty, immorality \\
\hline Simplicity, impulsiveness, short gameplay, triviality, nonchalance & Complexity, commitment, time investment, diligence, discipline \\
\hline Cannot die or lose, no obligations or objectives & Achievement, progress, competition \\
\hline Short learning curve, low effort & Mastery, learning, high effort \\
\hline Real world, familiarity, concreteness, conventional & $\begin{array}{l}\text { Imaginary, fantastical, abstraction, novelty, } \\
\text { culture, artistic interest }\end{array}$ \\
\hline Practicality, repetition, habits & Aesthetics, variation, intellect, thinking \\
\hline Expectedness, routine, continuity, clear boundaries, rules & $\begin{array}{l}\text { Adventure, discovery, surprise, curiosity, choice, character } \\
\text { development, storyline }\end{array}$ \\
\hline Single/solo play, reserved, solitary, low social engagement & $\begin{array}{l}\text { Team play, cooperation, company, crowds, high social } \\
\text { engagement }\end{array}$ \\
\hline Low interaction, non-communicative, passive, receptive & High interaction, communicative, assertive, expressive \\
\hline $\begin{array}{l}\text { Low stimulation, low focus, low activity, } \\
\text { laid-back gameplay, short attention span }\end{array}$ & $\begin{array}{l}\text { Physical, sensory, high focus, high activity, } \\
\text { action, continuous attention }\end{array}$ \\
\hline Moderate, slow-paced, no time pressure, calm gameplay & Intense, thrilling, fast, time pressure, exciting gameplay \\
\hline Relaxing, comforting, calming, pleasant & Suspense, anxiety, panic, frustration, inducing stress \\
\hline Confidence, control, clarity, safety & Vulnerability, helplessness, fear, shock, confusion, danger \\
\hline Cheerful, positive, unrestrained, cuteness & Compulsiveness, depression, humiliation, gloom, horror \\
\hline
\end{tabular}

\subsubsection{Classification}

Below follows a description of each domain and its main characteristics. The characteristics of the 6D model are intended as a comprehensive clarification of the domain extremes. Semantics may therefore be simplified or elaborated to accommodate for its intended target group.

Conflict - Conflict expresses the nature of game content in terms of conflict with other players or the environment. It distinguishes friendly from violent game content. Harmony is characterised by altruism, pacifism and integrity, while the opposing extreme discord is characterised by violence, destruction and rivalry. Players scoring high on this domain may derive satisfaction from the opportunity to behave immorally.

Dedication - This domain expresses the dedication the game demands of the player. Casual game content is characterised by for example simplicity, short and nonchalant gameplay, lacking negative consequences for mistakes. Devoted game 
content is characterised by demanding discipline or effort (also time investment) to progress, allowing for achievement and competition, having a higher overall complexity.

Novelty - Novelty describes the content that may satisfy the player on an intellectual level versus a more practical orientation. Conventional content is characterised by resemblance to the real world, but also by clear rules of the gaming environment, routine and repetition, familiarity and expectedness. Novel content, on the other hand, is described by a high level of imagination and abstraction, artistic interest such as music and aesthetics, the absence of boundaries and how much the game allows for adventure and discovery.

Social - This domain expresses the amount of player-to-player interaction in a game, or the satisfaction derived from the presence or absence of social engagement. Solitary is characterised by (in social context) passive, receptive gameplay while engaged is by expressive, assertive gameplay. Team play and cooperative play are also characteristics of engaged game content. It must be noted that a multiplayer game can be rated as solitary when there is no communication or active social engagement.

Intensity - Intensity describes the amount of exertion the game poses to the player. Slow-paced game content is characterised by low activity lalso a low number of actions), absence of time pressure, a short attention span and a low speed. Exciting game content is fast and thrilling, demands high focus (such as multitasking) and a high number of actions that determine gameplay.

Threat - Threat expresses the capacity of the game content to give the player emotions normally considered negative. Low threat, calm, is characterised by the feeling of safety and comfort, cheerfulness, the deliberate intent of the game to create a pleasant experience. High threat content, stressful, is described by the presence of tension and suspense, feelings of vulnerability, danger and frustration. A depressing atmosphere, horror and frightening elements can also cause this.

The expert sessions furthermore resulted in an additional list of motivational factors. These factors were considered an essential background to the motivation to play from in-game content, and may be relevant in future developments. However, they cannot be classified using our framework, are out of scope for this study and are addressed in research already. The identified factors were autonomy, immersion (including escapism and relaxation), nostalgia, peer behaviour and promotion. These factors can describe the (hedonic) effect games have on the player, such as the feeling of autonomy and immersion, including escapism and relaxation, which is different from relaxed, laid-back gameplay or deliberately relaxing game content [4 1-46]. Also, 'nostalgia' (games from your youth, retro games) was seen 



Figure 2 - Visualisation of $95 \%$ interval of domain scores by all users $(n=91)$ and subgroups on gamer percentage

as a factor that currently heavily influences motivation [47]. Furthermore, external factors can be environmental influences such as social context lgames that friends or peers play, possibility of sharing or joining in, games that certain communities play) $[48,49]$ and external promotion (games that stores recommend, (extensive) advertising, or personally being susceptible for games that are 'hyped'). 


\subsubsection{Survey}

User preferences are assessed through the survey (see box). The survey items measure how satisfying a set of proposed game characteristics are for a player. Users can express their preferences by scoring each a number of statements, using a 7-point VAS scale (using a slider in the online version). Each statement presents a set of opposing game characteristics from one domain. In total, all content is rated through 16 survey items, using clustered game content characteristics. From the expert sessions, we found that four domains required three questions and two domains required two questions to be properly measured.

The assessment of the utility of the survey by the panel of experts resulted in the following. The survey was found to sufficiently represent the game content of the classification (answered yes in 100\% of cases). The survey items were found to adequately assess the intended game content or the preference for this content lyes in $100 \%$ of cases). The domains were found to be represented equally respective to each other (yes in 100\% of cases). The content validity ratio of all items was measured by the experts rating the importance of each question to the construct being measured, from essential to useful but not essential to not necessary. All items scored a content validity ration (CVR) above $0.7(M=0.94)$. We therefore conclude that the utility of the survey is sufficient to form accurate and useful user or game profiles, and that it can be used in practice in a user evaluation study.

\section{3| Practical demonstration study}

\subsection{Methods}

In the second section of this study, we performed a practical demonstration study in real-life setting in order to show the insight that is gained from applying the 6D framework in practice. We deployed the framework survey online and invited users of games to participate. We analysed the preferences of this group, explored if subgroups could be identified and if their game profiles were significantly different. Additionally, we explored if the framework can be used to recommend suitable games to selected users. The relations between the user profile and the game profile of the most preferred game from the previously constructed list of games were investigated.

\subsubsection{Participants}

Participants are people interested in (video)games. Participants were recruited online via an invitation asking for people who enjoy games. The invite, containing a direct URL, has been distributed via colleagues, friends, acquaintances, internal company and university networks, via e-mail and advertisement on social media (Facebook, Twitter, Linkedln, Steam discussions forum) and a local video games store. Participants were asked to spread the invitation within their networks as well. Data was collected through online survey service SurveyMonkey. 
Table 1 - Domain scores of all users and subgroups

\begin{tabular}{l|lrrrrrr} 
& \multicolumn{1}{|c}{ n } & \multicolumn{1}{c}{ Conflict } & Dedication & Novelty & \multicolumn{1}{c}{ Social } & Intensity & \multicolumn{1}{c}{ Threat } \\
\hline All participants & 91 & 42.7 & 67.5 & 69.2 & 53.2 & 56.2 & 38.9 \\
\hline Age $<25$ & 15 & 48.5 & $80.7^{* *}$ & 74.5 & 52.2 & 64.4 & 44.1 \\
Age 25-50 & 63 & 41.1 & $64.4^{*}$ & 68.9 & 52.9 & 54.6 & 38.7 \\
Age 50> & 13 & 44.0 & $67.1^{*}$ & 65.0 & 55.8 & 54.5 & 34.2 \\
\hline Gamer $>75 \%$ & 32 & $47.2^{* *}$ & $74.3^{* *}$ & 73.4 & 58.3 & $67.2^{* *}$ & $43.1^{* *}$ \\
Gamer 50-75\% & 28 & $45.8^{* *}$ & $70.8^{* *}$ & 72.0 & 53.9 & $57.7^{* *}$ & $44.0^{* *}$ \\
Gamer <50\% & 31 & $35.3^{*}$ & $57.3^{* *}$ & $62.4^{*}$ & 47.3 & $43.5^{* *}$ & $30.1^{* *}$ \\
\hline Daily & 41 & $46.5^{* *}$ & $75.1^{* *}$ & 70.9 & 54.9 & $61.2^{*}$ & $40.8^{* *}$ \\
Weekly & 20 & $44.7^{* *}$ & $64.2^{* *}$ & 70.6 & 51.3 & $60.8^{*}$ & $42.2^{* *}$ \\
Monthly & 14 & $50.0^{* *}$ & $65.1^{* *}$ & 75.0 & 51.8 & $53.0^{*}$ & $49.2^{* *}$ \\
\hline Hardly ever & 16 & $24.3^{* *}$ & $54.2^{* *}$ & 58.3 & 52.6 & $40.6^{*}$ & $21.2^{* *}$ \\
Male & 66 & $48.5^{* *}$ & $71.0^{* *}$ & 68.5 & 52.8 & $58.5^{* *}$ & $43.0^{* *}$ \\
Female & 22 & $23.2^{* *}$ & $54.5^{* *}$ & 72.2 & 54.9 & $46.6^{* *}$ & $25.2^{* *}$
\end{tabular}

Table 2 - Profile correspondence score per domain (participants scoring within a $+/-10 \%$ range), number of participants that consider the domain influential in their game selection

\begin{tabular}{|c|c|c|c|c|c|c|}
\hline$n=80$ & Conflict & Dedication & Novelty & Social & Intensity & Threat \\
\hline profile match & $48 \%$ & $64 \%$ & $55 \%$ & $40 \%$ & $40 \%$ & $50 \%$ \\
\hline not influential & 41 & 18 & 30 & 17 & 15 & 35 \\
\hline influential & 41 & 64 & 52 & 65 & 67 & 47 \\
\hline
\end{tabular}

\subsubsection{Materials}

Through an online survey (www.surveymonkey.com/r/gamepref), we measured: 1) demographics and gaming behaviour, 2) preferences for game characteristics in six domains, and 3) preference for existing games through creating a ranking of 10 games. The survey was made available in Dutch and English. As experience with video games influences if the participant can relate to the content presented in the questionnaires, a self-assigned level of acquaintance with (video)games is determined through the survey. Information on gaming behaviour of the participant included general acquaintance with (video)games such as asking how much the participant regards him or herself as a gamer ('Are you a gamer?', 0\%-100\%), gaming frequency (daily/almost daily, often/at least once a week, occasionally/ at least once a month, hardly ever/never), playing session duration (seconds, less than 15 minutes, 15-30 minutes, hours, days), preferred devices ((portable) console, smart device, pc/laptop) and variation in games played by the participant 10-100 scale from 'always different' to 'always the same'). 
Game preferences were assessed by measuring satisfaction derived from proposed game characteristics using the 16-item survey as previously described. In addition, the participant was asked to indicate whether each domain was considered or not in their game choice (yes/no) when they looked for a new game. The preference for existing games was then measured by presenting the user 10 games off the previously described game list, as rated by the experts. From the original 65 games, 4 unique sets of 10 games were created. This was done to reduce the eventual load for the participants to complete the survey. Each of these sets of games has similar consistency and variation regarding the different games' contents. The game lists are randomly assigned to the participants. Participants were asked to arrange these 10 games on how likely they would play them (from 1 (highest) to 10 (lowest)). In this study, we analyse the two games participants indicate to prefer.

\subsubsection{Data analysis}

Data analysis was performed using Statistical Package for Social Sciences (SPSS v.22). Data for each participant was sorted for the six domains of the 6D classification. The 16 survey items were converted to a percentage and from these the six average domain scores were calculated. We choose to present these domain scores in this article, while calculating average scores results in a loss of finer nuances on the selected game characteristics. This results in a 'user profile' for each participant.

Subsequently, we explored these user profiles for different subgroups. Variables in the analysis to identify these subgroups are demographics age (in groups), gender (male and female), gaming frequency and self-assigned gamer score, which were investigated through comparing means. Data distributions of all demographics and the scores on the six domains were analysed using the descriptives of skewness and kurtosis, visual analysis of boxplots and histograms. Data were found normally distributed for all participants. Subgroups were identified by comparing mean scores on the six domains and analysing significance levels between subgroups within the same variable, using the t-test for parametric distributions. In order to differ between groups, subgroups were identified in comparing means in age, self-assigned gamer percentage score, playing frequency and gender. Age was partitioned in 1) younger than 25 years, 2) 25-50 years and 3) 50 years and up. The gamer score was partitioned in $110-50 \%$, 2) $50-75 \%$ and 3/ $75-100 \%$. Gamer score was partitioned as such to ensure an even distribution of participants along groups. Univariate linear regression analysis was used to study the explained variance in the domains by all subgroups. The principal findings are presented in a visual overview using the domain scores of all participants as well as the most striking subgroup(s), based on the highest number of significantly different domain score means. This overview provides insight in the score means but also in the range. 
Finally, we analysed the relation between the domain scores of the user profile and the domain scores of the top preferred game profile. By doing so, we aim to determine whether we can recommend specific games to users based on our own insights (or the insight of an expert panel) using the 6D framework. The differences between the scores on each of the user profiles and of the selected game profiles are calculated. We then investigated correlations between the profiles per domain, calculated the percentage of correspondence of both profiles for all participants, and the percentage of participants that has a good match between profiles per domain (range $+/-10 \%$ ). By counting the number of times each game has been top ranked we identify a possible difference in overall popularity. The top ranked game for each of the four sets of ten games were counted. Along all games, the average number of times they were ranked highest was 1,8 (standard deviation 1,3). One outlier was identified with a count of 9 (the game Assassin's Creed).

All correlations were explored with Pearson using a significance level of $\alpha=0.01$ unless indicated otherwise. Correlation strength is interpreted as follows: $r< \pm 0.1$ is little or no correlation, $\pm 0.1 \leq r< \pm 0.3$ is a weak relation, $\pm 0.3 \leq r< \pm 0.5$ is a moderate relation and $r \geq \pm 0.5$ a strong relation.

\subsection{Results}

The survey was filled in by 102 participants (98\% within Europe). Participants were aged $14-68$ years $(M=34$ years), $72.5 \%$ of participants was male $(n=74)$, $24.5 \%$ was female $(n=25)$ and $3 \%(n=3)$ identified as other. Of all participants, $64 \%$ identified as a gamer with a score of $50 \%$ and up (of which $8 \%$ female). Of the female participants, 15 people identified as a gamer with a score of $25 \%$ or lower. The English survey was used in $51 \%$ of the cases. About $70 \%$ of participants has completed higher education. For all participants as well as all subgroups, pc/ laptop is the most preferred medium to play (all $n=59$ ), followed by console $(n=$ 20 ) and mobile devices $(n=19)$. A daily gaming frequency was indicated by 43 participants, 23 people play at least once a week, 15 people play at least monthly and 21 participants indicated to rarely play. Hour-long or more playing sessions was chosen most often $(n=51)$, followed by $15-30$ minutes $(n=32)$ and up to 15 minutes $(n=15)$. Three participants indicated their playing sessions were merely seconds in duration, and one participant indicated to play for days (combined with an occasional playing frequency). Data was excluded for participants indicating to rarely/never play combined with playing time of 15 minutes or shorter $(n=7)$.

The survey returned 91 complete 'user profiles', consisting of six domain scores (table 1). The user profiles and $95 \%$ interval of the gamer percentage groups were illustrated in a graph (figure 2), as this subgroup shows the largest number of significant differences and is most influential based on linear regression. This gives the designer more insight in the range of scores and the overlap of scores that exist between subgroups in determining a suitable design strategy. The means and range of the $50-75 \%$ and $>75 \%$ group's scores on all domains are closer together, while intensity is an exception. 
All participants scored below neutral on conflict as well as on threat. This may indicate that their preferences are more towards moderate or non-violent content and that they have lower tolerance for content that induces negative emotions. A significantly lower score for these domains is found for the lower self-assigned gamer percentage group, the lower playing frequency group and the female participants. We see a score for all participants on dedication and novelty that is on the higher side of the spectrum. For different age groups, we found that the score for dedication stands out, which indicates that younger players prefer game content that for example enables achievement and demands commitment to play.

The score on dedication decreases with decreasing gamer percentage and playing frequency. Novelty is scored higher than neutral by all participants, and while higher for the younger group we find no notable differences among subgroups for this domain. The scores in the domain intensity show the same pattern as in the other domains: a decreasing score for decreasing gamer score and playing frequency, and female participants. Participants are not particularly outspoken about the social component, which for all participants as well as for any subgroups is scored around $50 \%$. It must be noted that while a normal distribution of scores is expected, this does not mean that a score of $50 \%$ can be generalised as the average for the entire population on each domain.

The means of subgroups divided by self-assigned gamer percentage ("are you a gamer?") significantly differ for all domains except novelty and social. In general, a higher gamer percentage results in a higher score on all domains. It must be noted that the majority of female participants are in the lower gamer percentage groups. The scores on conflict and threat are particularly low for the lowest gamer percentage group, indicating a preference for non-violent content and a lower tolerance for content inducing negative emotions. We investigated the means for different playing frequencies, and see that scores are similar to the gamer percentage (these variables strongly correlate). Observing scores for male and female participants, we see an evident lower preference for conflict and threat and a higher dedication score for male participants. Again, novelty and social are the only domains not resulting in significantly different scores.

In order to investigate whether we can recommend existing games to users based on their profile and our (expert) analysis of these games, we explored the correlations between the profiles of the chosen top two games and the respective user profile $(n=80)$. Moderate correlations $(p<0.05)$ were found for all users, between the profiles of the user and best-rated games, on the domains conflict $(0.41, p<0.01)$, novelty $(0.30, p<0.01)$ and threat $(0.45, p<0.01)$. The number of times each domain was considered of influence by a participant in selecting a new game is shown in table 2. We see that conflict was found the least often influential to game selection, while intensity was most often considered. The correspondence per 
domain, calculated from the number of participants scoring their preferred game's content within $a+/-10 \%$ range of their user profile, is shown in table 2 . The correspondence (inverted average difference) between the scores per domain of each individual user and their respective top ranked game was between $51 \%$ and $93 \%(M=76 \%)$.

\section{4| Discussion}

This study worked towards a framework for game developers to gain an initial understanding of their target audience's preferences for game content. With this framework, we meet the need for a practical method that supports the development of game-based applications outside of entertainment purposes. The 6D framework extends the current classifications that are used in industry frameworks by being applicable to a broad audience, enable context-specificity as well as show user motivation in the form of profiles. Demonstrating the framework in practice in this article was done by applying it to a general audience, and resulted in overall scores on six domains of game content as well as an indication of the composition of preferences of a target audience through subgroups. In this article, we chose to elaborate the results of the survey in summarised format, by means of a graph on the six domain scores. Detail from the original 16 items is not retrievable from this graph but may be needed for interpretation of the results to form game design recommendations. A challenge now lies in the translation of data resulting from applying the framework into real game design, and future research should focus on the extension of the framework to create game design recommendations.

The framework presents several challenges. It enables us to classify what usually motivates an individual or group of users, or the contents of a game from a single perspective. However, it is evident that both people and games are often much more complex and dynamic. People often have more than one profile, more than one mood or mind-set to play, and may be influenced by many other external factors that, as we found in the expert sessions, cannot be addressed by the classification. Similarly, games may be more complex too and offer multiple play styles, as intended by the game (for example missions versus sandbox) or even emergent gameplay (invented by the user), which would lead to more than one profile. Certain favoured actions or behaviour may classify as a combination of characteristics from more than one domain (for example exploration or strategy). We avoided such terms in the classification, as they lean towards genericity found in genre classification. While the insight resulting from a mere 16 items may not be fully complete or accurate, it gives a more dimensional insight in the end-user than other methods we are aware of. Also, we are not convinced that more detailed information is a guarantee for better insight in the target group, or actual game design resulting from this information - which can only be explored in practical follow-up studies. 
The framework cannot distinguish between an indifference towards content on a domain, or a neutral or even preference for both extremes, when a user scores $50 \%$. In that case, content on a certain domain may not play a role in the motivation of a user, but preference may be just as well in the middle or even indicating an alternation of both extremes in one game (for example an optional multiplayer, or strict missions next to discovery). An example of this is found in the preferences of participants on the domain conflict. We see that while the higher gamer percentage groups score higher on conflict, we would expect to find this value to be higher overall as well as for the $>75 \%$ gamers, as is the case for intensity. We can speculate that this may point towards an indifference towards the presence of absence of violence in games from the higher gamer percentage groups, perhaps from being more used to higher levels of violence or being triggered more by other domains such as intensity. Exactly half of the participants indicated that this domain did not influence their selection of new games, while all the other domains were valued much more often than not, supporting this assumption. The lower gamer percentage group may indeed have a stronger aversion against violence, therefore resulting in a lower mean score. In future research, it may be worthwhile to extend the framework with a method to capture these nuances.

The overview shows us why a one-size-fits-all approach for content on most of the domains would have a high chance of failure to engage the end-user, even in a group that is quite homogeneous. Also, the study demonstrates where certain nuances lie for the different subgroups which we can use to determine which game characteristics would contribute to an appealing game for this particular target audience or a subsection thereof. We can for example see that while a higher intensity game may motivate people in the higher gamer percentage groups, it will most probably not be attractive to anyone from the lower percentage group. A game that is within the acceptable range of threat for the lower gamer percentage group would less likely motivate the more seasoned gamer. Younger people, for example, may need a more demanding, challenging game than the rest of the population and a high conflict or threat game may put off the more incidental player.

The data resulting from the study furthermore enabled us to compare profiles of user preferences with profiles of game content. We made this comparison to investigate if we could successfully match existing games with users, based on the user profiles and the game profiles that we compiled in the expert sessions. We investigated the match between the two profiles and found an average of $76 \%$, up to a best match of $93 \%$, which we find promising. The percentage of users that had a very close match between the scores on each separate domain varied from $40 \%$ to $64 \%$. Correlations found were not yet convincing. Future research may shed more light on improving the accuracy of this prediction by overcoming some of the following challenges. As games may have been chosen for different reasons than we can 
identify using the framework, this may lead to a bias when trying to match new game content or an existing game to the preferences of a user. Furthermore, it is likely that the presented games have a difference in overall quality (i.e. a 'better' game may be automatically ranked first). Lastly, to reduce the load on the user, four groups ranked a different set of games. This enabled the ranking of a larger number of games, but resulted in relatively smaller correlations. While the match between most preferred game and user profile was promising and inviting for more research, we find that the main limitation in recommending existing games to a user is the lack of insight in the way the end-user experiences the same content. In the demonstration study, the game profiles were set by a group of experts to reduce the load of asking the user to rate ten or more (possibly unknown) games on their contents. However, it is very well possible that each participant would create game profiles that are completely different. This is particularly important to take into account when the enduser is a less regular consumer of video games, such as the older adult, whom may have an entirely different frame of reference regarding game content. Therefore, the discrepancy between user experience and appreciation of game content should be measured. This gives the game designer insight in what content motivates the user, but also to what extent their perception on this content differs.

We have identified several limitations to the use of this framework regarding the interpretation of data resulting from its application. Firstly, some aspects of this study lead to limitations when generalising our findings for a greater public, particularly in use for game-based (serious) applications. For there is not a single typical user of serious games, their application being very diverse, we focused on a general public. However, our recruitment method attracted a majority of participants that finished higher education. Social background and education level may influence outcomes, and the user profile resulting from this study may not be applicable elsewhere. Also, since many of the participants scored high as self-assigned gamers, we assume that this group had a thorough understanding of the survey items and semantics used to address game content. Other target groups may have lesser insight in the semantics, possibly from limited experience with games, or a lesser interest in games altogether. The complexity of the survey items, in terms of semantics used, may therefore need to be optimised to fit the target group's experience and knowledge. While we have done so in an earlier study towards the preferences of children (De Vette et al., 2018), further research on the effects of adapting the survey items is needed. Furthermore, the majority of participants was male, and the majority of female participants indicated a below $25 \%$ gamer score. In this demonstration study, we found that the self-assigned gamer score is the most important predictor of game preference. We therefore focused on the values we found for these subgroups. While we are in favour of looking beyond gender to assess user preferences in games, we do not assume that other variables should be ignored. 
We carefully state that our first efforts towards investigating if the framework enables recommendation of full existing games to users based on their profiles were successful. Besides its intended application in game-based design for serious purposes, we hope that in the future this framework will enable consumers of video games to express their preferences more in-depth and select new games accordingly, as well as inspire game developers to explore and address new markets and target groups. Lastly, we want to emphasise that without a clear context of its intended use, including the external factors that were identified in the expert sessions, these findings cannot readily be translated into practical recommendations for serious applications. Other factors such as specific usability requirements, time and location of play, devices used and perhaps certain health issues may affect how the measured preferences lead to concrete game design. It must also be noted that the quality of a game is key to motivation in general. It is unlikely that an entertaining game can be created based solely on measured preferences, without a creative process involving skilled designers. 

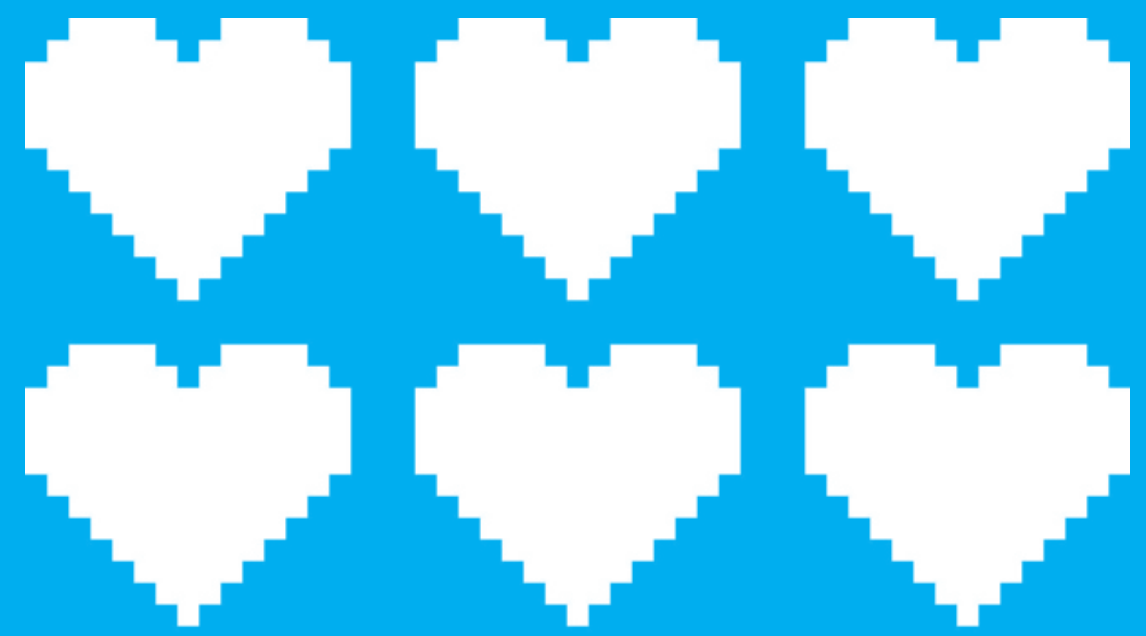
Chapter 6| A gamified physical activity coaching application for older adults: design approach and user experience in daily life

Submitted 


\section{Abstract}

Objectives: In this paper we describe the design approach of a game-based mobile coaching application that aims to stimulate physical activity (PA) in daily life. We evaluated the older adults' experience with this application compared to a standard coaching application in terms of engagement in using the application, motivation to be physically active, and in relation to the applied design features.

Methods: A user-centred design approach has led to the game-based coaching application, called WordFit. Step count data (FitBit) was used as input for the crossword-inspired game. The standard coaching application was the ActivityCoach, which displays FitBit data. Participants were asked to use the ActivityCoach for one week, followed by up to 3 weeks WordFit. Engagement was determined by logging actual use, and motivation for PA by a daily in-app motivation question and step count data. Afterwards, a semi-structured interview was conducted.

Results: Twenty older adults participated (71.0 \pm 5.0 years). 16/20 actively used the ActivityCoach and 8/20 used WordFit. For the latter, mean PA before was $5852 \pm 3652$ steps/day, while mean PA using WordFit was $7236 \pm 3335$ steps/ day. The interviews $(n=12)$ showed that step data and feedback on performance through the ActivityCoach/FitBit was the prominent motivator for PA. WordFit was generally played as a stand-alone brain trainer. Cognitive challenge, ingame challenges and challenges through emergent game play were important for engagement to use. Older adults did not play WordFit together.

Discussion: WordFit was not seen as a tool to help improve PA behaviour. The enjoyment of the game concept could be improved by providing older adults with new and non-traditional gaming concepts prior to assessing game preferences. To explore the added value of game-based coaching over traditional coaching, follow-up studies should be conducted in a target group that is known for low adherence rates and that can benefit from the intervention provided.

\section{1| Introduction}

Deploying a more active lifestyle is considered important for older adults, as physical activity (PA) is consistently associated with e.g. lower mortality rates, higher levels of cardiorespiratory and muscular fitness, higher levels of functional health and better cognitive function [1]. Despite these well-established benefits for health and wellbeing, the majority of older adults does not meet the recommended levels of PA $[2,3]$ and leads a sedentary lifestyle [4]. Consequently, a growing number of interventions that promote daily PA emerge, including technology-supported coaching applications for daily life. 
Intervention studies regularly report high levels of dropout and limited use of the technology-supported applications [5-8]. As stated by the WHO: "Medicine will not work if you do not take them" and assuming a dose-response relationship between the engagement of the user and the effects on the intervention [9], it is likely that engagement of behavioural interventions in general could positively affect treatment outcomes [ $10-11$ ] . Ongoing communication efforts (e.g. telephone contacts) that keep the patient engaged in interventions may be a simple and costeffective strategy [12], which is also commonly applied in eHealth interventions $[13,14]$. Gaming technologies may offer a more efficient alternative to increasing engagement in using the technology, e.g. through incentives [1 1]. However, the use of game-based applications for behaviour change for older adults remain scarce $[15,16]$.

In gamification, engagement is supported by the integration of elements and mechanisms borrowed from games. Simple reward systems, like points, appear to be a foundational feature in game-based applications $[15,17]$. Following the Self Determination Theory, such reward systems that utilise extrinsic motivation could possibly inhibit intrinsic motivation and could therefore even counteract the establishment of sustained behaviour change [18]. To understand and analyse the interplay between engagement with the technology and behaviour change, Yardley et al. [19] make a distinction between the micro level of moment-to-moment engagement with the technology-supported intervention and the macro level of engagement with the intervention goals for behaviour change, which are closely connected. Detailed understandings of the features that influence engagement for both use of the technology and to motivate changes in behaviour are important for the development of the rapidly growing field of game-based coaching applications. However, the overlapping conclusion of recent papers is the incomplete description of the game-based interventions and how game features are operationalised $[17,19]$ and that the influence of such game features on actual engagement is not yet established [20].

In this paper we describe the design approach and evaluation of a game-based mobile coaching application that aims to stimulate physical activity in daily life of older adults. The application combines behaviour change techniques with gaming strategies to facilitate engagement with the intervention and target health behaviour objectives simultaneously. We evaluated the older adults' experience with this application compared to a standard (not game-based) coaching application in terms of engagement in using the application and motivation to be physically active. With our study, we aim to contribute to the knowledge-base of tailoring game design to the older adult's preferences and experience in daily life. 

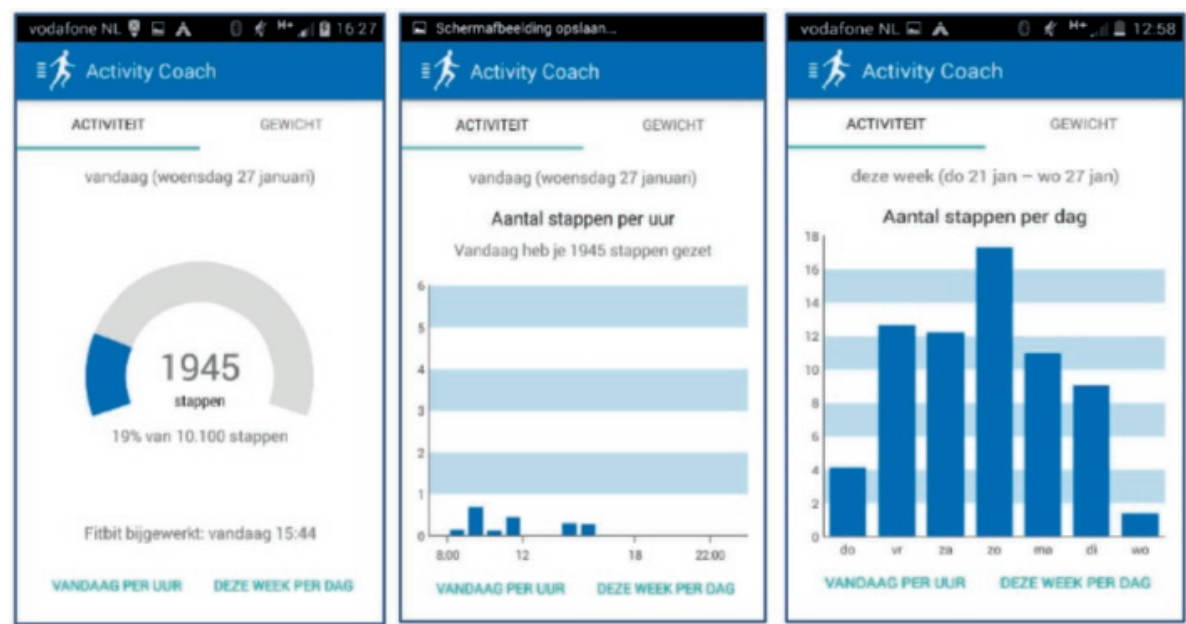

Figure 1 - GUl of Activity Coach

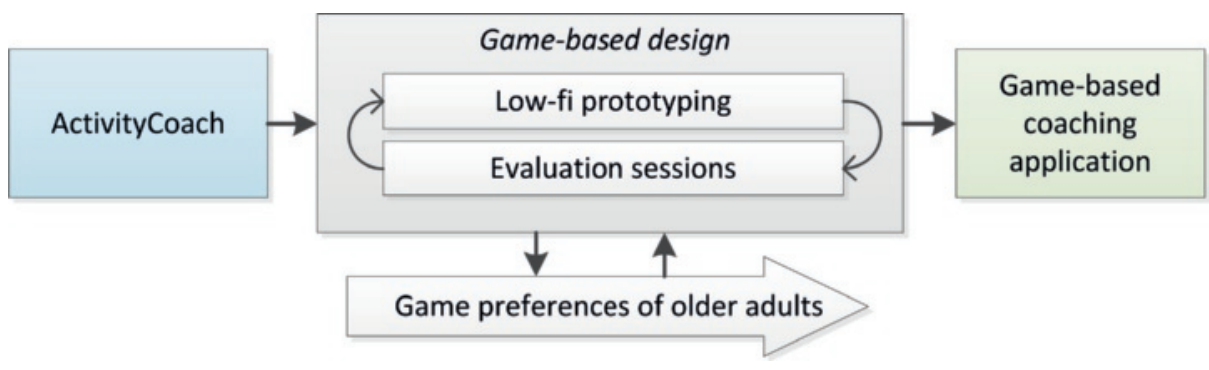

Figure 2 - Design approach for development of the game-based coaching application

\section{2| Development and design of a game-based coaching application}

\subsection{Standard application: Activity Coach}

The Activity Coach is a mobile phone application developed at Roessingh Research and Development, Enschede, the Netherlands, which aims to monitor and coach clinical and non-clinical populations into healthy active lifestyles [21]. The activity behaviour is monitored by the Fitbit Zipß step counter, worn in the pocket, which assesses number of steps throughout the day. A smartphone is used for providing feedback to the user. Users can see in real time the current number of steps in relation to their goal, distribution of steps today per hour and last week per day (Figure 1). The PA goal was set manually by the healthcare professional via a web portal, preferably in consultation with the user during a regular consult. In addition, the therapist could see an overview of the participants and their activity behaviour as measured by the FitBit in the webportal. 


\subsection{Design approach game-based application}

An iterative design approach has led to the development of a game-based coaching application (Figure 2). Starting point for development was the existing Activity Coach application, which has proven to be effective for several target groups (e.g. [22-24]], but needs improvement in terms of use and adherence rates [6].

First, we investigated the gaming preferences of older adults, incorporated these in several low-fi prototypes, which were evaluated with experts and older adults. In addition, research was done on how to apply specific game mechanics and features to the preferences of the older adult [25], of which the first results were implemented in the game-based application.

\subsection{Game preferences older adults}

To explore the current 'gaming behaviour' (i.e. gaming, gamification, and play; digital and non-digital) of older adults, including gaming preferences, gaming frequency, gaming context and motivation to play games of older adults, we performed an exploratory survey among 159 adults aged between 50 and 91 years (mean $66.9 \pm 9.2$ years) of which $44 \%$ males. $75 \%$ of the respondents played games (digitally $(39 \%)$, non-digitally $(36 \%)$ or both $(25 \%)$ ) and were invited to complete the whole questionnaire. In summary, we observed the following context for gaming of the older adult:

1. Devices: older adults prefer mobile devices and pc/laptop to play digital games on (rather than consoles such as XBOX)

2. Playing motivation: the most important reason to play a game is for relaxation ( 108 times) followed by to train the memory (59 times) and for challenge (42 times). A combination of these factors is appreciated.

3. Social interaction: players prefer playing with others (43\%), alone (39\%) and against others (18\%). A balance of competition and cooperation within games is appreciated.

4. Game type: older adults mostly enjoy puzzle-type games, often based on conventional games, for example Yahtzee, Rummikub, and Scrabble.

In addition, we investigated which game characteristics should be applied to improve engagement for this specific target group [25]. From this ongoing research, the following first results were taken into account in the development of the game-based coaching application:

5. The older adult is achievement-striving (rather than playing out of habit) and game content should satisfy their need for feedback on progress and successes. Also, competition is highly valued, including challenging themselves. The older adult enjoys putting effort in a game to complete objectives. 


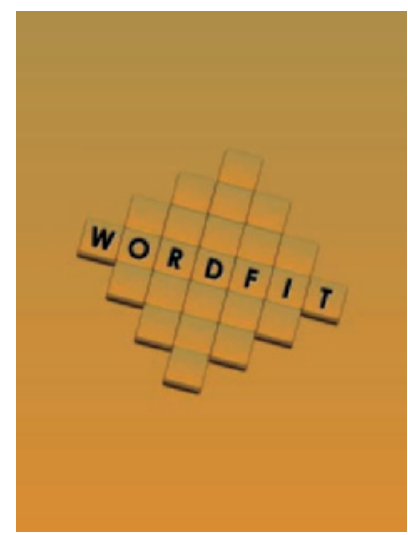

Splash screen

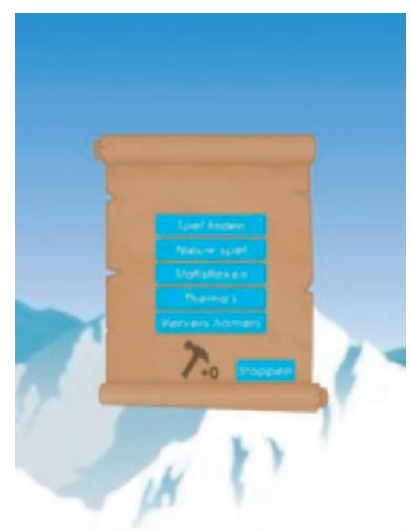

Main menu

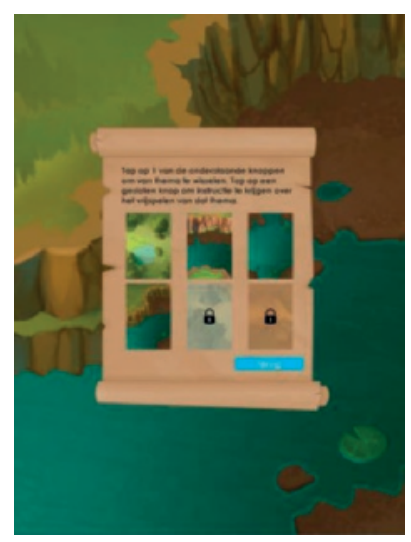

Unlock themes

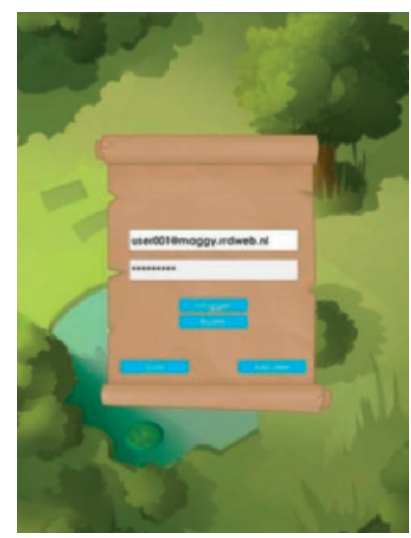

Log on screen

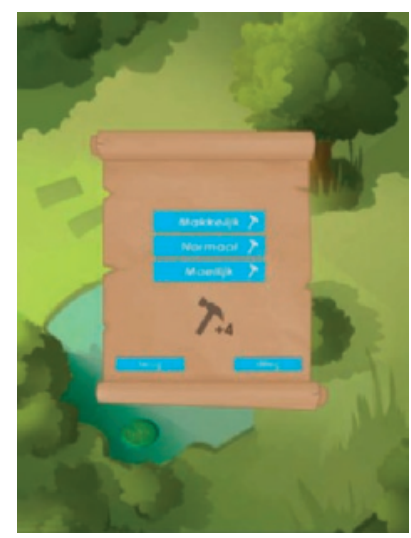

Choose difficulty level

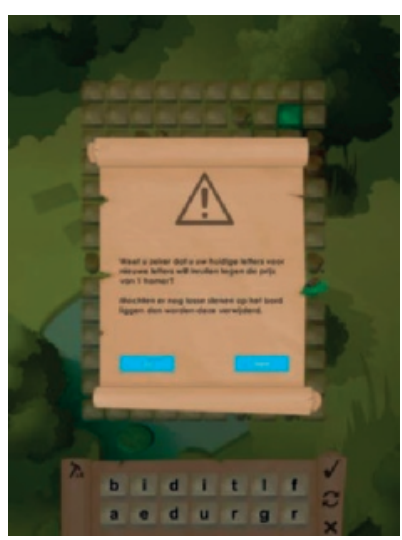

Trade new board for hammer

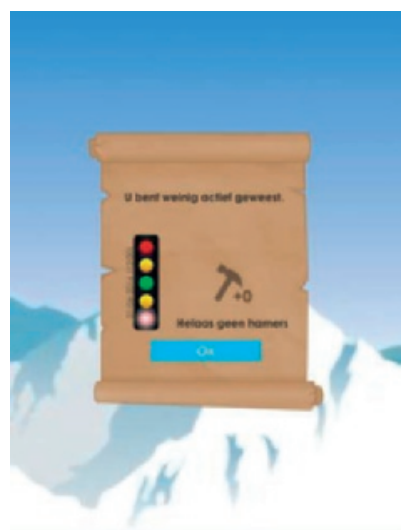

Activity feedback

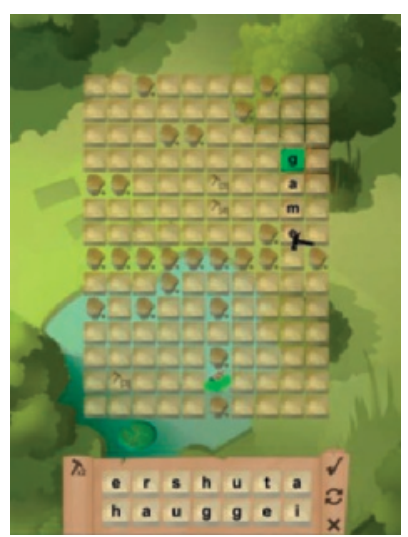

Game board

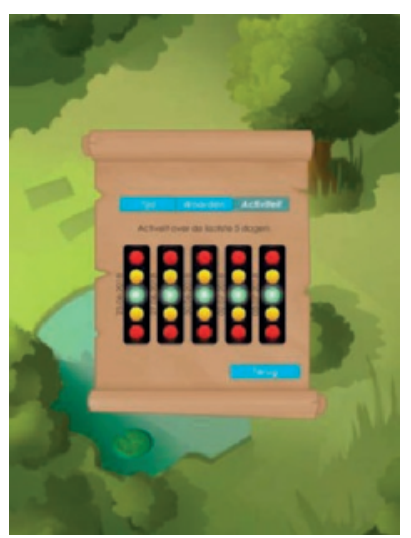

Scores: Activity overview screen

Figure 3 - Screenshots of WordFit with different themes shown 
6. The older adult enjoys variation in game content. Challenges should be made interesting by means of new content and lead to various outcomes. Games with a high aesthetic value are appreciated.

7. Problem solving and puzzle-themed games are found to be highly motivating. The target group is however open to modern games and new experiences.

8. Older adults are unspecific in their interest in interaction with others in or through digital games, while social contact is highly valued through traditional (board/card) games.

9. Older adults are easily demotivated by fast and stimulating gameplay, and have a low tolerance for stressful and frustrating content. A trialand-error approach is hardly ever taken and leads to loss of interest in the application or game. Frustration may also be avoided by enabling pausing and resuming the game at any point.

10. The older adult strongly dislikes any expression of violence.

\subsection{Game-based coaching application: WordFit}

The design approach has led to the development of the game-based coaching application - called WordFit. WordFit is a prototype game (Figure 3) to engage the older adults in use of the technology and thereby supports the older adult in deploying and maintaining an active lifestyle (Table 1). The abovementioned preferences (no. 1 to no. 10) are incorporated as follows. Progression in the game depends on measured daily activity. The game is designed to be played on the smartphone or tablet, anywhere, anytime (no. 1), alone or with others (no.3, no.8). The game concept is a crossword puzzle, inspired on conventional crossword puzzle games such as Scrabble (no.2, no.4, no.7, no. 10). The main game mechanic is to place words from a starting point to an end-point (no.2, no.9). Rocks are placed on several tiles on the board, which need to be demolished by hammers in order to place words to reach the end-point. The hammers can be earned when the step goal, measured by the sensor, is reached by the user (no.5). In-game, extra hammers can be collected by placing words on special tiles. Hammers are also used to rotate or swap one or more letters with new ones (no.6). The user can open the game whenever he/she wants, although the hammers are only updated 3 times a day.

The game concept is a crossword puzzle, to be played on mobile devices. The main game mechanic is to place words from a starting point to an end-point. Rocks are placed on several tiles on the board, which need to be demolished by hammers in order to place words to reach the end-point. The hammers can be earned when the step goal, measured by the sensor, is reached by the user. Strategies are provided 


\section{Table 1 - Main characteristics of WordFit}

\begin{tabular}{|c|c|}
\hline Health topic(s) & Physical activity \\
\hline Targeted age group(s) & Older adults (65-75 years) \\
\hline Short description of game idea & $\begin{array}{l}\text { WordFit is a game-based application } \\
\text { connected to an underlying coaching } \\
\text { application (ActivityCoach). WordFit aims } \\
\text { to increase engagement of the older adult } \\
\text { user with the application, thereby supporting } \\
\text { the older adult in deploying and maintaining } \\
\text { an active lifestyle. The main game concept } \\
\text { is a crossword puzzle that can be played } \\
\text { on mobile devices. Physical activity as } \\
\text { measured by a sensor drives in-game } \\
\text { mechanics. }\end{array}$ \\
\hline Target player & Individual (optional guest or buddy player) \\
\hline $\begin{array}{l}\text { Guiding knowledge or behaviour } \\
\text { change theory(ies), models, or } \\
\text { conceptual framework(s) }\end{array}$ & $\begin{array}{l}\text { Self-monitoring of PA to create awareness, } \\
\text { goal setting, feedback on performance. } \\
\text { Cognitive challenge, social play, enjoyment, } \\
\text { achievement, autonomy. }\end{array}$ \\
\hline Intended health behaviour changes & $\begin{array}{l}\text { Physical activity behaviour, performance } \\
\text { towards personal goal. }\end{array}$ \\
\hline Knowledge element(s) to be learned & Insight in PA behaviour \\
\hline $\begin{array}{l}\text { Behaviour change procedure(s) } \\
\text { (taken from Michie inventory) or } \\
\text { therapeutic procedure(s) employed }\end{array}$ & $\begin{array}{l}\text { Persuasion and incentivisation within } \\
\text { WordFit. The application was part of regular } \\
\text { care where other additional procedures } \\
\text { le.g. education, trainingl were used to } \\
\text { achieve the health goals. }\end{array}$ \\
\hline Clinical or parental support needed? & Yes, physical therapist \\
\hline Data shared with parent or clinician & Yes, through web portal, showing PA \\
\hline Type of game & Puzzle, strategy, \\
\hline
\end{tabular}


Game components

\begin{tabular}{|c|c|}
\hline Player's game goal/objective & $\begin{array}{l}\text { Obtain as many hammers as possible to } \\
\text { finish puzzles. Solve and create challenges. } \\
\text { Improved activity behaviour enhances } \\
\text { rewards and creates and enables more } \\
\text { satisfying gameplay. }\end{array}$ \\
\hline Rules & $\begin{array}{l}\text { Rocks are placed on several tiles on the } \\
\text { board, which need to be demolished } \\
\text { by hammers in order to place words to } \\
\text { reach the end-point. The hammers can be } \\
\text { earned when the step goal, measured by } \\
\text { the sensor, is reached by the user. Themes } \\
\text { and challenges can be reached in game. } \\
\text { Creative, emergent gameplay is stimulated. } \\
\text { Elements enabling achievement are present } \\
\text { through leaderboards, which can be shared } \\
\text { with other users. }\end{array}$ \\
\hline Game mechanic(s) & $\begin{array}{l}\text { Gaming motivation, to obtain hammers and } \\
\text { enable reaching in-game goals, is translated } \\
\text { into real-world motivation to be physically } \\
\text { active. Performance is directly drives the } \\
\text { game's progression. Specific strategies and } \\
\text { implemented features are shown in Table } 2 \text {. }\end{array}$ \\
\hline $\begin{array}{l}\text { Procedures to generalise or transfer } \\
\text { what's learned in the game to } \\
\text { outside the game }\end{array}$ & $\begin{array}{l}\text { WordFit aimed to create sustainable } \\
\text { engagement with the technology and } \\
\text { thereby creating a positive user experience in } \\
\text { monitoring and adopting healthy behaviour } \\
\text { in daily practice. }\end{array}$ \\
\hline
\end{tabular}

Virtual environment

\begin{tabular}{|l|l|}
\hline Setting & $\begin{array}{l}\text { PA is measured throughout the day with an } \\
\text { activity sensor, WordFit can be played on } \\
\text { mobile devices in any environment. }\end{array}$ \\
$\begin{array}{l}\text { Game platform(s) needed to play the } \\
\text { game }\end{array}$ & $\begin{array}{l}\text { Smartphone or tablet } \\
\text { Sensors used } \\
\text { Estimated play time }\end{array}$ \\
FitBit zip to measure PA (steps). \\
$5-20$ minutes per day
\end{tabular}


Table 2 - Overview of the engagement strategies incorporated in WordFit

\begin{tabular}{|c|c|c|}
\hline & Gamification & Behaviour change \\
\hline Strategy & $\begin{array}{l}\text { Enjoyment } \\
\text { Autonomy } \\
\text { Emergent gameplay } \\
\text { Social play } \\
\text { Achievement }\end{array}$ & $\begin{array}{l}\text { Awareness of activity behaviour } \\
\text { Goal setting } \\
\text { (Feedback on) performance } \\
\text { Positive reinforcement }\end{array}$ \\
\hline $\begin{array}{l}\text { Gamification } \\
\text { features }\end{array}$ & $\begin{array}{l}\text { Cross-word puzzle } \\
\text { Unobtrusive rewarding (hammers) } \\
\text { Social accounts (buddy, guest) } \\
\text { Game statistics, personal leader board } \\
\text { Unique playing boards } \\
\text { Unlockable themes } \\
\text { Challenges (to unlock themes) } \\
\text { Difficulty levels } \\
\text { Expandable dictionary }\end{array}$ & $\begin{array}{l}\text { Traffic light metaphor represents PA } \\
\text { Review at past week's activity in } \\
\text { statistics } \\
\text { Linking back to original ActivityCoach } \\
\text { for detailed activity data } \\
\text { Goal achievement (optional: goal- } \\
\text { setting via portal) } \\
\text { In-game feedback messages on } \\
\text { activity performance }\end{array}$ \\
\hline
\end{tabular}

in table 2. In more detail, WordFit incorporates a combination of gaming strategies and behaviour change techniques to facilitate engagement with the intervention and target health behaviour objectives simultaneously, as discussed below and summarised in Table 2.

\section{Gaming strategies for engagement}

The enjoyment of the gameplay itself is seen as the main strategy for engagement by gathering hammers from measured PA to proceed in the game. As we aimed to avoid explicit extrinsic rewards (e.g. through point systems) more unobtrusive reward systems are applied to the game. Positive rewarding is applied by offering more hammers when players are closer to their personal goal. The better the activity behaviour, the better the award, the more satisfying the gameplay.

A second important strategy is autonomy, a player can freely choose playing approaches e.g. through different difficulty levels, or deciding to unlock certain challenges for changing the theme. This feeling of being in control is reinforced by the right of the user to add words to the library of the game. Furthermore, boards can be saved and re-opened at any time. Autonomy is also reinforced by enabling emergent gameplay, playing a game outside its intended rules. For example, upon 
finishing a board, some game statistics are displayed such as time spent finishing this level and number of words used. Players are thereby triggered to invent their own gameplay and rules, which may lead to a more meaningful experience.

These in-game statistics may also be compared with others to invite for competition. This third strategy, social play, is supported by the possibility for peers or friends to log into one of two extra accounts implemented in the game. A 'buddy' may log in with unique credentials and play WordFit under the same conditions as the main player, thus copying the activity level of the primary user. The rationale behind this is that the buddy can support and participate in the daily activity level of the primary user off line. A guest account is available for free play with random activity levels, in case a player doesn't want to share the activity data.

The game allows for achievement/skill development through the game statistics and a personal leader board, including time played to finish a game, words and hammers used, played difficulty levels, and activity of the past week. Three difficulty levels are implemented: the higher the level, the higher the number of rocks present on the board. To satisfy the need for variety in the game, each time the game is started a new, unique board is created. Secondly, optional achievements are created by challenging the user to complete a game within the set specifics. For example, a challenge can ask to finish a puzzle on the highest difficulty level within sixty seconds. These challenges also satisfy the need for variation, as they are rewarded with new backgrounds for the application. Challenge may also emerge from social play and use of the buddy or guest accounts, stimulating engagement to both the game and the activity goals.

\section{Game-based coaching to motivate for physical activity}

Following principles of behaviour change theories, the game-based coaching application provides self-monitoring of the activity behaviour to create awareness, facilitates goal setting, and provides feedback on performance.

To provide game-based feedback, the PA data from the ActivityCoach is used as input for the game. After logon, a traffic light appears every time the app is started by the player to provide insight in the activity behaviour in the game (awareness). The player should be active according to his/her personal goal (goal-setting) that was set by the physical therapist via a web portal. Data is aggregated over predefined intervals, typically 00:00 - 12:00, 12:00 - 17:00, and 17:00 - 20:00 and is compared to the prescribed goal activity for that interval.

PA could be too low $(\geq 20 \%$ deviation below goall), slightly too low $1 \geq 10 \%$ deviation below goal), OK (<10\% deviation), slightly too high ( $\geq 10 \%$ deviation above goal), or much too high ( $\geq 20 \%$ deviation above goal). This is subsequently mapped to a five-point scale (-2 through 2). In game the activity level throughout the day was 
visualised by means of a metaphor with safety margins - a traffic light $10=$ green, $-1,1=$ orange, $-2,2=$ red). In addition, the traffic light is accompanied by a short message about the activity behaviour (feedback on performance) and consequently, how much hammers the user receives (positive reinforcement). These messages to support the older adult are friendly, short, generic and without strong arguments. In addition, the player can see an overview of the measured activity levels of the past 5 days, in traffic lights. WordFit offers a quick link to the Activity Coach from the game if more detailed information on the measured steps is desired.

\section{3| Methods}

\subsection{Study Design}

The aim of the evaluation study was to evaluate the older adults' experience of the game-based application compared to a standard coaching application in terms of engagement in using the application and motivation to be physically active. The study setting is in the daily life of the older adult, under supervision of the physical therapist at a distance.

\subsection{Participants}

Participants were eligible for inclusion if they fulfilled the following criteria: 6575 years old upon inclusion, and no health-related complaints that limit the performance of physical activities in daily life (able to comply to step goal). Participants were recruited from the physical therapy practices PMI Rembrandt, Veenendaal and De Klomp, Enschede, and the physical therapy departments from elderly care organisation Trivium Meulenbelt Zorg in Almelo, Hengelo and Borne, the Netherlands. The study was approved by the University of Twente Ethical Committee and all participants provided their written informed consent.

\subsection{Protocol}

The total study duration was four weeks. In the first week, participants were asked to use standard application ActivityCoach, which connects with Fitbit. In the following weeks, participants were asked to use WordFit for as long as they wanted, with a maximum duration of 3 weeks. The daily step goal was set to a standard of 6000 steps/day, and could be altered through the webportal by the physiotherapist to set a personal goal that fitted the capabilities of the individual participant. The participant was free to choose if, when and how often the applications were used.

\subsection{Measures}

To determine engagement, objective use data was automatically gathered for the FitBit, the Activity Coach and WordFit and saved in log files, from the first to the last measurement day. A day of use was defined by the presence of step data and counts at least 100 steps; otherwise the day was not used for analyses. One day 
of use in the Activity Coach was counted when the app has been opened. One day of use of WordFit was counted when at least one word has been placed on the board within the game.

The motivation to be active was measured by experience sampling on a daily level, by asking on a scale of 1 to 7 each day on the mobile device. In addition, the FitBit was used for registration of PA and expressed in steps/day.

Semi-structured interviews after the study aimed to provide detailed information on the user experience in terms of engagement with the technology, motivation to change activity behaviour and overall experience with both applications and the game features incorporated.

\subsection{Data analysis}

For engagement, the successive use days, mean (SD) number of use days and the amount of PA is presented for the FitBit, Activity Coach and WordFit. We stratify these outcomes for active ( $\geq 2$ days of use) and non-active users (0-1 days of use). Application use is graphically presented over the course of the study. For the motivation score and PA data, the mean (SD) is presented separately for the days before using WordFit and for the days WordFit use was started. As this was an exploratory study to investigate user experiences and not (clinicall) effects, no power calculation was done beforehand, and no statistical analyses are performed.

The interviews were transcribed and thematic content analysis was applied by two main authors separately. The number of times unique participants mentioned game features (as mentioned in Table 2) as either motivating or demotivating was counted.

\section{4| Results}

\subsection{Participants}

Twenty-one older adults were recruited, one person dropped out before the start of the study due to illness. Twenty older adults participated in the study (mean age $71.0 \pm 5.0$ years). Eight older adults dropped out before the end of the study (unknown reason $(n=2)$, not compatible with lifestyle $(n=1)$, too much effort $(n=1)$, becoming overly active $(n=1)$, illness $(n=1)$, private event $(n=1))$, meaning that 12 older adults participated in the semi-structured interview held after 4 weeks.

\subsection{Engagement in using applications}

The total sum of successive use of all participants was 466 days, with a mean successive use of $23.3 \pm 11.0$ days. Engagement in using the applications varied greatly: all participants used the FitBit, 16/20 participants used the standard Activity 
Table 3 - Participant characteristics ( $n=20,1$ missing data)

\begin{tabular}{|l|l|}
\hline Age & $70 \pm 5.0$ years $(n=19)$ \\
\hline Gender (male/female) & 10-Oct \\
\hline Stage of change & Precontemplation: $n=0$ \\
& Contemplation: $n=1$ \\
& Preparation: $n=4$ \\
& Action: $n=2$ \\
& Maintenance: $n=11$ \\
\hline Technology use & Daily: $n=8$ \\
& Weekly: $n=0$ \\
& Monthly: $n=3$ \\
& Rarely: $n=3$ \\
& Never: $n=4$ \\
\hline
\end{tabular}

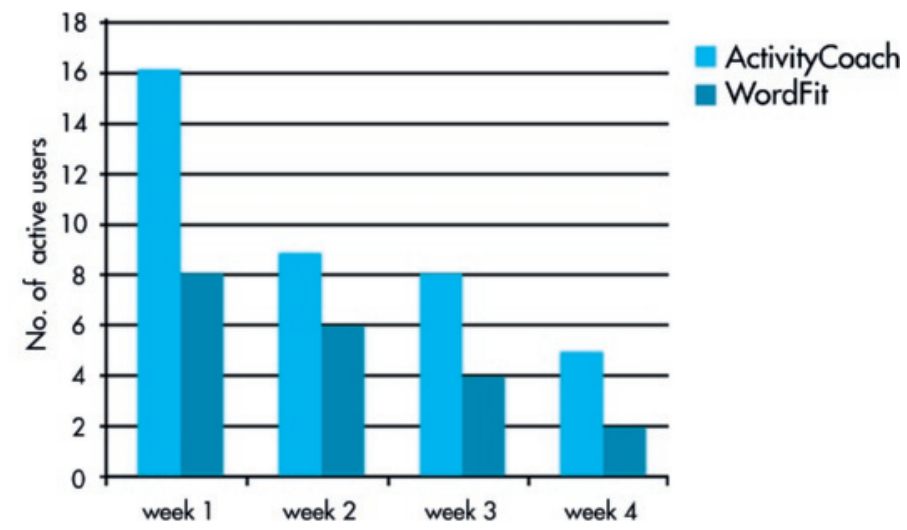

Figure 4 - Number of users per week, stratified per application

Coach application of which 7 participants also played the WordFit game. One participant played WordFit, but did not use the Activity Coach. 4/20 participants used the FitBit without the applications.

Mean use of active users $(n=16)$ was for the Activity Coach 13.1 $1 \pm 10.0$ days (range: $2-36$ ) and mean use of active users $(n=8)$ for WordFit was $14.8 \pm 9.5$ days (range 4-32). Figure 4 shows the number of active users per week, per application. This figure also shows that participants did not follow the protocol saying that WordFit should be used from week 2 onwards. The buddy and guest accounts were rarely used. 


\subsection{Motivation to be physically active}

Ten participants answered the daily motivation questions. The mean motivation to be physically active for the days before starting using WordFit was rated $4.8 \pm 1.5$ $(n=53)$ and $6.0 \pm 1.2$ for the days after starting using WordFit $(n=140)$.

The overall mean daily PA level for the days before starting using WordFit was $5235 \pm 3389$ steps $(n=17)$. For the active WordFit users $(n=8)$, mean daily PA level for the days before starting using WordFit was $5852 \pm 3652$ steps, while the mean steps/day for the days after starting using WordFit was increased to $7236 \pm 3335$ steps. Six participants had measurements days before using WordFit and after using WordFit, of which 5 showed an increase in PA (range: 282-2353 steps) and one a decrease of 1700 steps.

\subsection{User Experience}

Twelve users participated in the interview, of which 6 were very active WordFit users (one to three weeks [p 1, p2,p3, p 1 8, p 19,p20]), 2 users were active users (max.one week [p4,pl0]), and 4 participants were non-active users [p14,p15,pl6,pl7].

\subsection{Motivation to be physically active}

The majority (9/12) of the interviewed participants [p1,p2,p3,p10,pl4,p15,p $18, p 19, p 20$ ] found the FitBit motivating to be physically active. Reasons were seeing the number of steps [p1,p2,p10,pl4,p15,p18,p19] and comparing their activity behaviour with previous days [p2, p 10,p19,p20] or with other persons [p10,p14,p15], which motivated them to put slightly more effort in being active each day. Five participants wanted to continue using the FitBit after the end of the study and considered buying one [p1, p2,p3, p15, p18].

One participant indicated that the number of steps does not represent your total daily activity very well [p 18]. Six users [p2,p3,p4,p 18, p19,p20] were motivated to be physically active by the ActivityCoach, because the ActivityCoach provided deeper insight in the daily activity behaviour $[\mathrm{p} 3, \mathrm{p} 18, \mathrm{p} 19, \mathrm{p} 20]$ and in relation to your goal $[\mathrm{p} 2, \mathrm{p} 19, \mathrm{p} 20]$.

Only two participants indicated they were motivated to be active because of the WordFit game [p3,p4]. "Just because you are working on your physical activity in a pleasant way helps you to become more active"[p3], while others were clearly not motivated [p1,p2,p10,p14,p15,p18,p20]: "This [WordFit] did not activate me. That would not help me at all, a game to motivate me to become more physically active. That was already clear from the beginning"[p 18]. Although the game did not motivate most, they would recommend the game to others $[\mathrm{p} 2, \mathrm{p3}, \mathrm{p} 10, \mathrm{p} 16, \mathrm{p} 18] . " Y e s$, it could work for other people, it really depends whether you like the game itself"[p19]. 
Figure 5-Overview of positive (+), neutral (+/-) and negative (-) responses of the interviewed participants
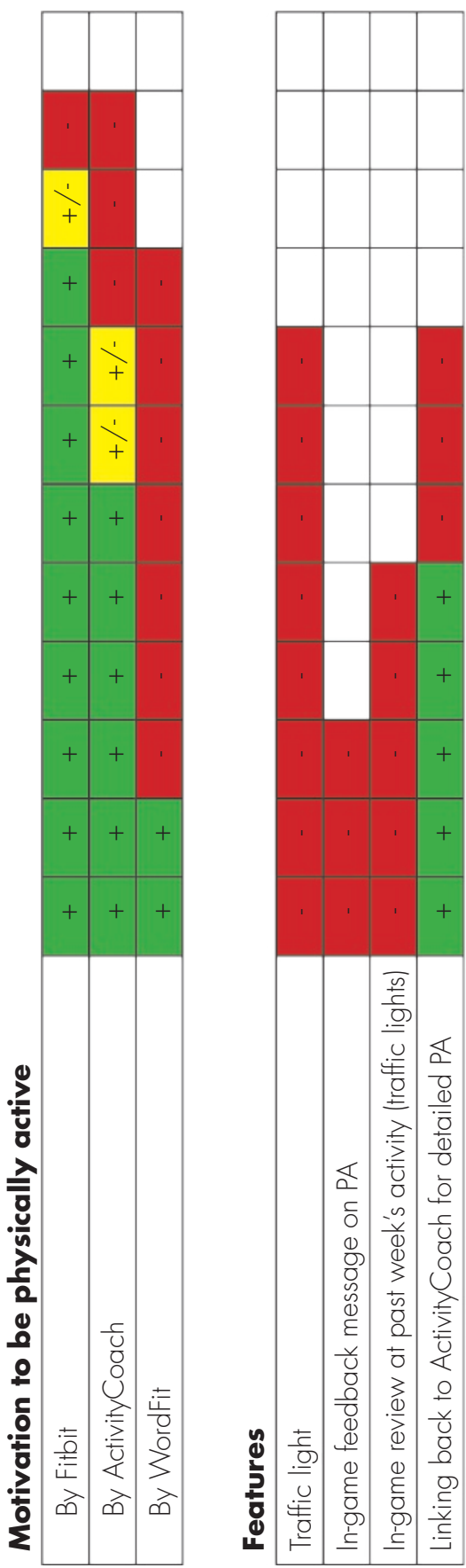

Figure 6 - Overview of positive $(+)$ and negative (-) experiences of the gaming features to increase engagement in using the WordFit application

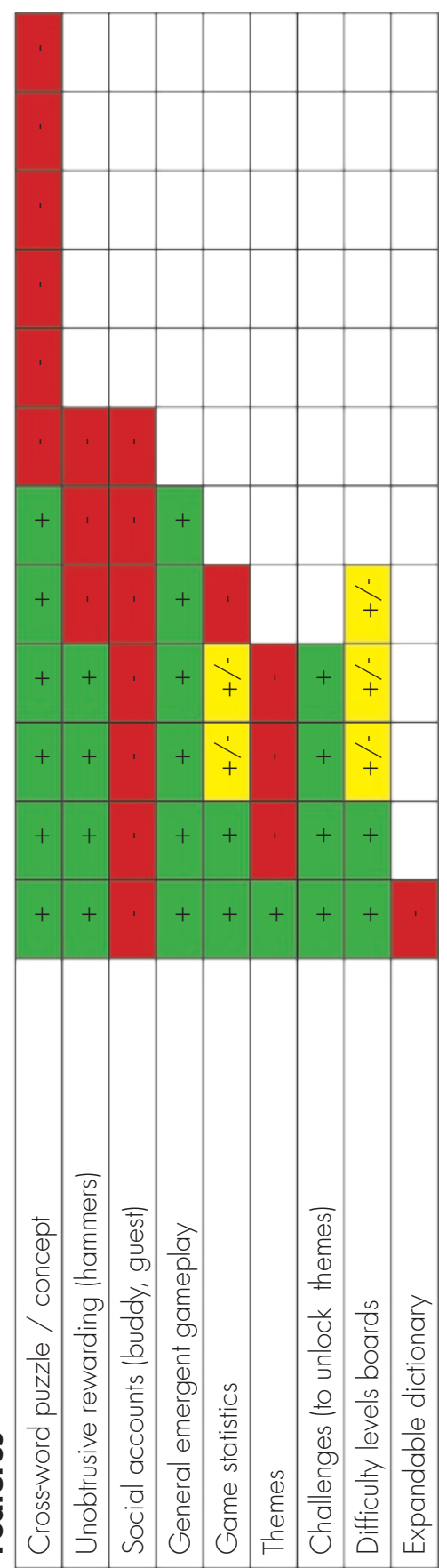


The connection between PA and the WordFit game in general, was not clear to four users $[p 1, p 3, p 4, p 19]$. The traffic light metaphor did not find a positive response among the participants. The traffic light was found unclear [p 1, p3, p18, p19,p20], incorrect $[p 2, p 3, p 18]$ or insufficiently informative $[p 1, p 2, p 3, p 18]$ as a direct message on activity performance as well as a means to review past weeks activity. "Information from the traffic lights alone was not enough [p3]". Users also indicated that the in-game feedback was unclear [p 1,p3] or too harsh resulting in annoyance[p1,p18,p20] "[the system said] 'too little activity, go do something', while I already walked 5800 steps and then I think: bugger off [p20]!" Linking back to the original ActivityCoach for seeing detailed activity data was regularly used by 5 participants [p2,p3,p18,p19,p20], while 2 other participants indicated they did not use it as seeing the steps was sufficient $[\mathrm{p} 4, \mathrm{p} 16]$.

The feature 'Goal setting' was indirectly present in WordFit, as it was a prerequisite for determining the colour of the traffic lights, while the feedback performance in relation to the goal could be seen in the ActivityCoach application. The latter was positively experienced by two users [p2, p3]. The standard goal of 6000 steps was not modified during the study [p2, p3, p18].

Social contact was also seen as a motivator for PA [p2, p10,p14,p15], however not as part of the applications but in real life "You talk about it with each other, about the number of steps you take every day, also when walking together. That's fun to compare". [p2]

\subsection{Engagement in using the applications}

Six users enjoyed playing WordFit [p1, p2, p3, p4, p19,p20]. In general they liked to play a few times a day, at home. "Yes, I liked it, just sit and play for a bit" [p2]. An important factor for the experienced enjoyment was the puzzle/brain trainer aspect $[p 1, p 2, p 3, p 4]$ and creating and setting challenges $[p 2, p 3, p 19]$. The persons who did not like to play WordFit mainly disliked the puzzle concept in general $[\mathrm{p} 10, \mathrm{p} 16, \mathrm{p} 17, \mathrm{p} 18]$. Too little challenge or variation in the game were the other main reasons not to enjoy playing [p 10,pl4,p18]: "It is too much like Scrabble" [p 10,pl6,p18].

The unobtrusive rewarding by the hammers was positively valued by four participants $[p 2, p 3, p 4, p 19]$. Five participants mentioned that the hammers were not needed for playing the puzzle and/or that it was too simple to obtain hammers in-game (cheating) by choosing easy levels [p1, p3, p18, p19,p20], thereby highlighting the multipurpose of the hammers. "It would have been nice if you could not earn hammers in the cross-word puzzle. That it would be more a reward for the no. of steps you take" [p20] and 'I started easy, then normal, then difficult, just to get the hammers'. [p 19] Some participants found it frustrating that the hammers were not saved for the next day $[\mathrm{p} 1, \mathrm{p} 2, \mathrm{p} 20]$. 
Seven participants indicate that the guest and buddy accounts were not used $[p 1, p 2, p 3, p 4, p 17, p 18, p 19]$ (confirmed by the log data). Strikingly, when asking whether they would like to involve family of friends in the game, six participants respond positively $[\mathrm{p} 1, \mathrm{p} 2, \mathrm{p} 3, \mathrm{p} 16, \mathrm{p} 19, \mathrm{p} 20]$.

From the applied game features, the challenges to unlock themes were valued most, while the expandable dictionary was valued least. People looked at the game statistics [p 1, p3, p4,p19] but they were not necessarily engaged by the game statistics $[p 1, p 4]$. "You see the statistics after playing, how long you played etc. and I looked whether I did better than the previous time" [p3]. Unlocking themes was experienced to be fun, although not for getting a new theme, but for doing the corresponding challenge [p 1, p2, p3]. Room for emergent gameplay, gameplay outside its intended rules, was generally found engaging. Participants set their own challenges, e.g. gathering as many hammers as possible [p 1,p19] as well as created their own cheating methods to receive more hammers [p 1, p 18, p 19, p20].

\section{5| Discussion}

WordFit was a first prototype game and research tool to explore the concept of a game-based application to support use of a behaviour change intervention. Our study showed that WordFit was not seen as a tool or addition to help improve activity behaviour. Instead, awareness of activity behaviour by seeing the number of steps and feedback on performance through the ActivityCoach and FitBit was the most prominent motivator for PA. The WordFit game was generally played as a stand-alone device for brain training. Challenge was the most prominent feature for engagement with the technology. With regard to our study, we observed the following factors that are beneficial for the experienced engagement and resulting success of the behaviour change intervention:

Design: Six of the twenty participants actively engaged in using the WordFit application, which is comparable to the study of Spook et al., in which $28 \%$ of the people used the Balance It intervention [26]. An important reason in our study was the lack of perceived enjoyment of the puzzle concept in general and that the connection between PA and the game was not clear to the majority of users.

These findings are contradicting our chosen design approach which incorporates user preferences and low-fi prototype evaluations. Due to the unfamiliarity of the envisioned technology, solely asking older adults about their preferences is probably not sufficient. In the study of Bird et al., exposure to and participation in a serious game showed a dramatic increase in the older adults' perception of the usefulness of technology for improving health outcomes including PA [27]. As such de Vette et al. 
provided older adults with a number of tablet games to play in daily life. Afterwards, the older adults' preferences for game content were mapped to the 7D framework, showing that older adults actually prefer new and non-traditional gaming concepts [25]. When we use this framework to map the game content of WordFit and compare this to the older adults' user preferences, we see the discrepancy of the medium to low novelty in WordFit and the preferred high novelty of the older adult [28]. In future design studies for new (gaming) technologies we therefore suggest to provide older adults with innovative gaming concepts prior to assessing their game preferences and let them more actively experience the technologies during the design process. This may lead to new insights in their gaming preferences, which are valuable in designing an engaging gaming concept.

Target group: The participants in this study were already highly motivated about increasing or maintaining their PA levels, and positively influenced by gaining insight in their actual daily activity in the form of step count during the study. As such, it is debatable whether the participants in our study needed the 'trigger' and extra motivational support of the applications and this could explain that WordFit was mainly used as a brain trainer game. Engagement can be seen as a dynamic process with four phases [19]. WordFit was mainly designed to trigger engagement with the technology intervention (phase 1) and thereby letting people experience the new behaviour (phase 2). However, our participants did not necessarily need the game-based intervention as they were already actively involved in their PA behaviour (phase 3), and WordFit would only be of added value for re-engagement with the technology in case of PA relapse (phase 4). This could explain that the standard coaching application showed higher engagement with the technology and the desired PA behaviour, thereby removing the need for additional motivational support through a game-based application. To explore the added value of game-based coaching over traditional coaching, follow-up studies should be conducted with a focus on clinical effects in a target group that is known for low adherence rates in the use of PA supporting applications and that can actually benefit from the intervention provided.

Coaching: The awareness of the activity behaviour through the step data and the feedback on performance (in relation to a goal) highly engaged the older adults to work on their activity behaviour. The in-game feedback on PA was unclear and insufficiently informative, and even counteracting user autonomy. This may have caused an early loss of interest in the game-based coaching app. In other studies, older adults emphasise the importance of continuous feedback on their performance from peers or instructors for engagement [29]. Interestingly, although indicated in the development phase, the option for social play was not used at all. The findings also suggest that, while social contact in conventional games is appreciated, 
older adults mostly prefer single-player digital games. The latter was also found in previous research on gaming preferences [30]. The continuous feedback from the FitBit could have fulfilled the older adults' need for continuous feedback making the gaming features more or less redundant for reaching engagement. A comparable result was found in the study of Muller et al., in which an interactive intervention to promote PA in diabetes type 2 showed lower engagement compared to the plain-text intervention [31]. On the other hand, older adults in this study and in literature29 indicate that social contact would be an important motivator for PA outside the gaming context, in daily life. It would be worth investigating how social interaction can be incorporated in technology to help each other engage in PA.

Challenge: In our study, both in-game challenges as challenges through emergent game play were perceived as important for engagement to play WordFit. Challenge has been found to be most popular motive to play a game (e.g. [30]). These challenges and emergent gameplay were intended to reinforce the feeling of Autonomy and Achievement of the player. This feeling could be improved by adding more variety in the game and improving some features (hammers, in-game feedback). Now, the older adults did not understand the connection between their PA behaviour and the WordFit game, and the hammers were disputing their feeling of being in control and lack of user autonomy. Despite the low perceived motivation of the active WordFit users, visual inspection of their PA data showed that 5 out of 6 even improved their PA behaviour (not statistically tested). A similar result was found for the Balance It intervention, where exploratory analyses suggested that "Balance It" could contribute to changing PA behaviours when used as planned [26].

Context of use: From previous research we know the importance of embedding the technology in the overall treatment program, with the utmost important role for the healthcare professional (e.g. $[32,33])$. In the study of Buimer et al., the behaviour of professionals showed to be an important incentive to use eHealth technology for rehabilitation as well as stimulation from fellow patients [34]. In our study, the physical therapists were informed about the study, but only a few people from their practice participated, and thereby the study was not part of their regular routine. In addition, the technology should be part of daily life in general and fit the habits of the older adult. Our results show that the majority of the older adults prefer to play at home, a few times a day, but mostly in the evening. This also accounted for the FitBit, which was mostly checked at the end of the afternoon or early evening. Engagement with the technology for behaviour change is influenced by many factors, including diverse target group characteristics, context of use and mode of delivery $[19,35]$, and their interrelations are complex. In future large-scale studies, clear communication to patients about the benefits of the technology, expectation management and the incorporation in the offline (group) training program and in daily life would probably stimulate engagement. 


\section{Strengths \& Limitations}

This study provides detailed insight in the implementation of design strategies and features for engagement. Future studies may benefit from the knowledge gained from this study regarding this group of older adult's game preferences. The users did not comply strictly to the protocol to first use the ActivityCoach for one week without WordFit. The study was exploratory and did not provide any statistical evidence.

\section{Lessons learnt}

From our research, we can distinguish several lessons learnt for designing a gamebased coaching application for older adults:

- Older adults do not necessarily like traditional game concepts for gamebased applications.

- Let older adults experience innovative related technologies to elicit the correct requirements; solely asking about their preferences based on their prior experience is not sufficient.

- In designing game-based coaching applications, the context of use should be carefully taken into account. For example, implementation strategies in daily routines (of older adults and healthcare professionals) should be designed alongside the application.

- Choose an appropriate target group to evaluate: people who are not motivated to play in general will not be motivated by a game-based application. People who do not need or want to change PA behaviour, will not use your application as intended.

- Feedback on PA should be highly informative and the role of the physical activity as input of the game should be extremely clear.

- The user wants a feeling of autonomy. Sufficient and adequate challenge is key to sustaining engagement.

- Social support could be of added value for engagement offline, for example by exercising together. 

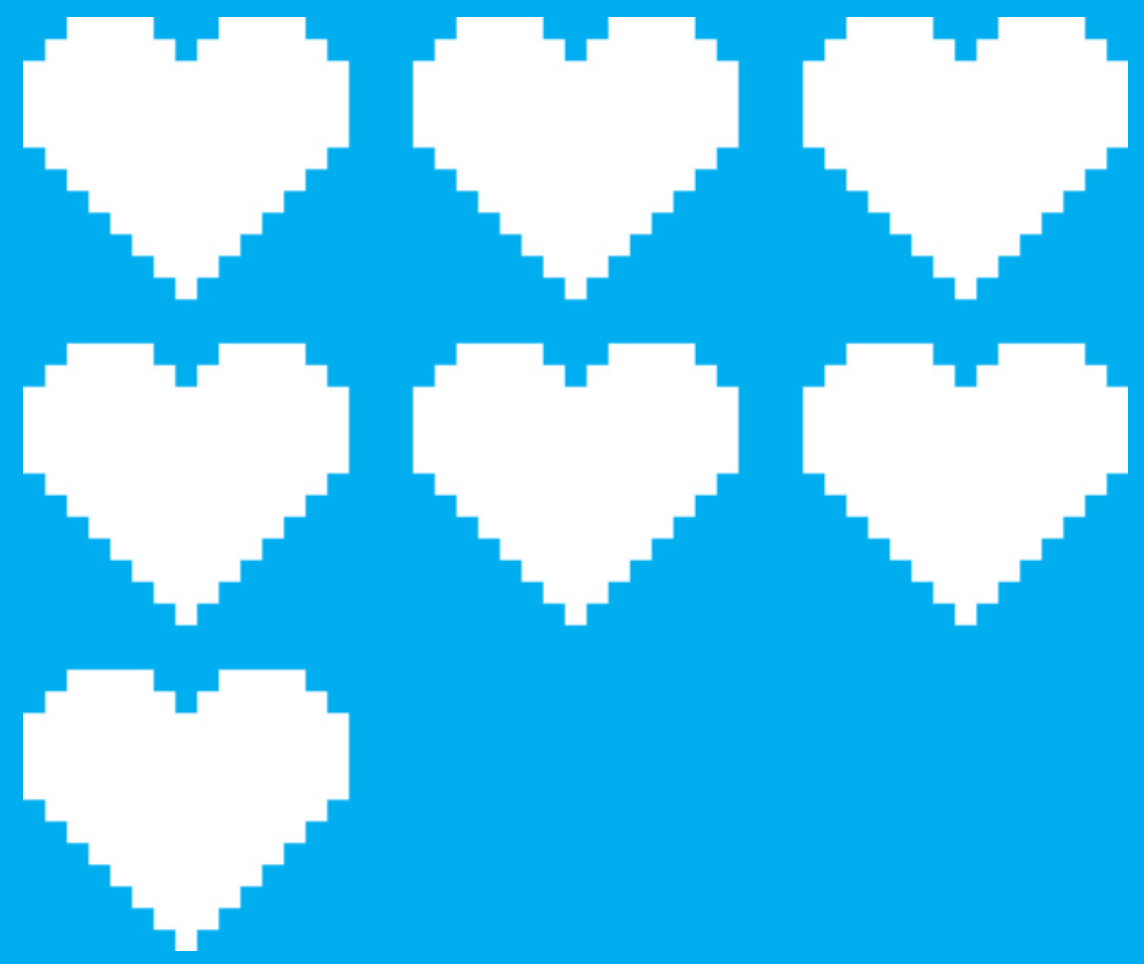


\section{Abstract}

Background - Games are increasingly used in eHealth as a strategy for user engagement. While these game-based applications receive attention in literature, there is an enormous diversity of end users and objectives targeted by eHealth. Identifying game content that drives and sustains engagement is therefore challenging. Future developments would benefit from more openness on the game design process and motivational strategies applied.

Objectives - Our objective was to provide insight in our approach in the development of game-based eHealth in practice. By means of a case study, PERSSILAA, we elaborate the entire game design process and show the motivational strategies applied, to aid researchers and designers of future game-based applications. PERSSILAA is a self-management platform which aims to counter frailty by offering older adults training modules in the domains of healthy nutrition, physical and cognitive training to maintain a healthy lifestyle.

Methods - We introduce four phases in the process towards game-based eHealth: 1) end-user research, 2) conceptualisation, 3) creative design and 4) refinement.

Results - A total number of 168 participants participated in end-user research (1), resulting in an overview of their preferences for game content and a set of game design recommendations. We found that conventional games currently popular among older adults do not necessarily translate well into engaging concepts for eHealth. Recommendations include: focusing game concepts on thinking, problem solving, variation, discovery and achievement, using high quality aesthetics. Stakeholder sessions with developing partners resulted in strategies for long-term engagement (2), using indicators of user performance on the platform's training modules. These performance indicators, e.g. completed training sessions or exercises, form the basis for game progression. Results from prior phases were used in creative design (3) to create the game "Stranded!". The user plays a shipwrecked person who has to gather parts for a life raft by completing in-game objectives. Iterative prototyping (4) resulted in the final prototype of the game-based application. A total number of 35 end users participated using simulated training modules. The online gamebased application was used without reported errors for a six weeks. End users scored appreciation (74/100), ease of use (73/100), expected effectivity and motivation (62/100), fun and pleasantness of using the application (75/100) and intended future use $(66 / 100)$ which implicates that the application is ready for use by a larger population.

Conclusion - The study resulted in a game-based application for which the entire game design process within eHealth was transparently documented. We believe we have contributed to the transfer of knowledge on game design that supports 
engagement in eHealth applications. Our user evaluations indicate that results from end-user research and consequential strategies for long-term engagement led to game content that is engaging to the older adult end user.

\section{1| Introduction}

Digital healthcare applications can contribute to improved self-management of patients and increased health literacy, alleviating the burden on healthcare professionals at the same time [1-5]. This eHealth, defined as the field in the intersection of medical informatics, public health and business, refers to health services and information delivered or enhanced through the Internet and related technologies [6]. However, maintaining adherence rates among users, related to better health outcomes [7], remains an issue [8,9]. Games, or elements from games (e.g. gamification, serious gaming, game-based design and applied games), are often used as a strategy for creating motivational concepts in an effort to stimulate engagement [10-12], thereby retain adherence of the end user to the objectives of these eHealth applications $[13,14]$. Over the past few years, these gaming strategies have evolved from a novel and experimental industry practice to a more mature field of research with applications in diverse domains [15]. As such, there is an expanding body of literature on studies towards the potential and effects of such 'gamified' applications $[16,17]$ while the broader adoption of games for eHealth is still in its infancy [18-21].

A general success formula does not exist for game-based design in eHealth, as the applications in eHealth target a diversity of specific goals and users. To develop successful gaming motivation strategies in these applications we have to overcome several challenges. Firstly, strategies that contribute to the success of a game-based application remain hidden when the rationale behind design choices does not receive attention in literature. We may be able to discover suitable game design strategies from these works by for example reverse engineering or mass analysing contents of existing applications. However, when the actual game design itself happens within a black box [22,23], a potential piffall is that successful concepts or theories lose their motivational capacities once borrowed and applied outside of their original context. There is a need to open up this black box of game design and bring research findings into practice in a useful way, which can aid developers of game-based applications in the selection of suitable content, principles or mechanisms.

A second challenge lies in creating game content that creates durable engagement to sustain motivation, as is crucial in gamified applications [24], to adhere to the health objectives. Often, developers seem to accomplish short-erm userengagement through extrinsic reward systems [1 1] as is suggested by gamification 
practice from industry (as for example [25]), which have dominated the field [26]. Game design should however be created for an optimal user experience in terms of aesthetics, usability and fun [12], and primarily be entertaining [27]. As such, the term gamification is controversial for reducing design to reward mechanisms to manipulate behaviour (as per the definition 'the use of elements from games in nongame context' [28] rather than designing an engaging experience for a productive purpose. By gaining insight in the preferences of the user, content can be tailored [29] to satisfy their motivational needs [30] which contributes to an engaging experience. This insight is gained through assessing the unique properties of the targeted end user and adequately address these through selecting game elements that are in line with these preferences, as well as carefully taking into account the context of use and health objectives.

The purpose of this article is to provide insight in a start-to-end design trajectory of game-based eHealth. We aim to overcome the abovementioned challenges by documenting the game design process and exploring design strategies for sustained engagement. We demonstrate this approach in a case study on an eHealth platform for the older adult, called PERSSILAA (PERsonalised ICT Supported Services for Independent Living and Active Ageing). PERSSILAA was developed to identify and counter frailty among older adults (see box). A particular challenge in this case was to sustain motivation of the older adult for long-term usage. The older adult is a generally underexplored target group in gaming [31]. We present the development of this game-based application from initial end-user research to the final version that is ready for use in real practice.

Frailty is a condition that affects many older adults. Frail older adults are at increased risk for development of disability, dementia and hospitalisation [32]. The condition is multifaceted and the major dimensions of the decline, that often occurs gradually and goes unnoticed for a long time [33], are physical and cognitive decline as well as malnutrition [34]. This vulnerability for decline is caused by a lifestyle that lacks stimulation on these three dimensions; a lack of sufficient mental stimulation, physical activity or healthy nutrition. Fortunately, when the decline is identified at an early stage - so-called pre-frailty - it can be slowed down or even reversed by offering suitable training on these three aspects [35].

Methods to detect (pre-ffrailty among older adults and offer them the right training have been successful but resource-intensive, and the demand for this specialised care is increasing by an ageing population in Europe. The PERSSILAA platform aims to enable older adults to independently work on their health targets [36]. By combining eHealth and community-based service, moving away care from institutions while the older adult gains autonomy [37]. 


\section{2| Methods}

\subsection{Game design process}

The game design process consisted of four phases as part of the healthcare application design and development process of the eHealth application (fig. 1). These four phases were:

1. End-user research phase: investigation of game preferences of the end user and specifications from the use context of the envisioned application

2. Conceptualisation phase: addressing system and application architecture and the conceptual development of long-term engaging game content suitable for the end user

3. Game design phase: performing the creative and constructive processes

4. Refinement phase: prototyping and user evaluations

Each of these phases is discussed as if chronologically occurred, but insights gathered during subsequent phases often lead to adaptations in earlier ones. Each phase consists of several sub steps, as shown in Figure 1.

For completeness, the full development cycle of the game-based eHealth application contains three more processes: planning, implementation and maintenance (fig. 1). Planning takes place prior to the healthcare application design and development, including the four phases of the game design process. Here, the need that the envisioned eHealth application will fulfil is recognised and analysed. For the PERSSILAA case, the choice for the development of a gamified application was made. Then, implementation (or operationalisation) and maintenance (or optimisation) follow. In implementation, the rollout of the developed gamified application for use in practice occurs. If necessary, training of primary and secondary end users (e.g. caregivers, family members) also takes place during implementation. Maintenance starts once the application is launched and stretches the total lifespan of the application. It covers service and support for the end user, as well as backend maintenance and keeping content up to date. During implementation and maintenance, pilot testing and data gathering on long-term usage may lead to new insights and new iterations in the design or content.

\subsection{Game design process phase 1 - End-user research}

\subsubsection{End-user characteristics and limitations}

User characteristics within the context and goals of the eHealth application are explored. This includes all aspects relevant to consider when creating the game design, such as computer literacy, experience with related technologies including games and specific usability requirements. 


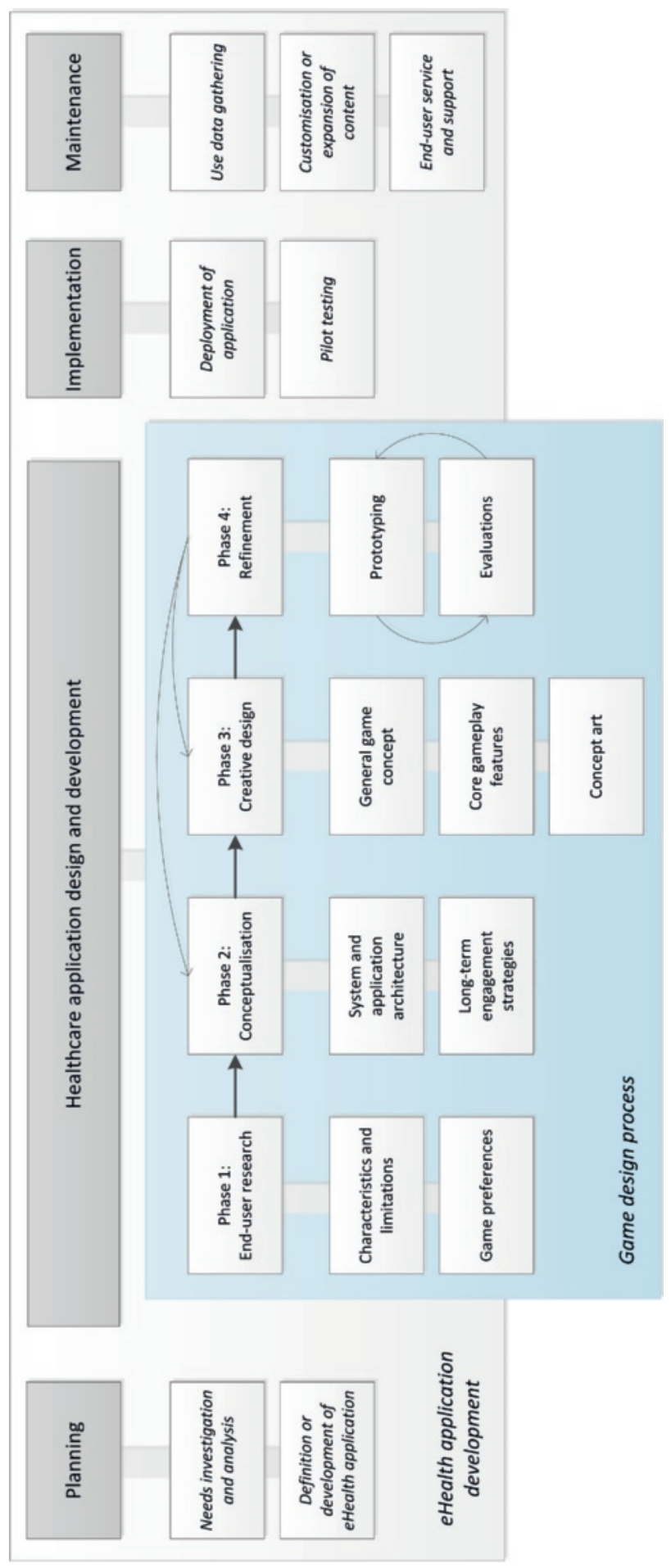

Figure 1 - Game design process within eHealth application development 
In the case study, we explored the characteristics of the target group eligible for the use of the gamified PERSSILAA self-management platform.

\subsubsection{Game preferences}

The preferences of the target user towards specific game content must be investigated to be able to design engaging gamification. In previous work, we have worked on a framework to assess and classify these preferences [38-40] land De Vette et al., 2019 (unpublished)). Assessment results in a 'user profile', subsequently translated into game design recommendations.

In the case study, we researched the specific game content that satisfies the older adult user. We reverted to a previously performed study [40], Study 1, and performed a follow-up study (Tabak et al., unpublished, 2019), Study 2. We combined the results to create an overview of the game preferences of the older adult regarding their gaming behaviour and preferences based on their current, prior and recent experience with (video) games. Also, we continued to elaborate findings by assessing game preferences before and after using a gamified eHealth application for several weeks that was developed specifically for this target group. The games and the gamified application were previously unknown to the participants.

In Study 7, we investigated the general game preferences of older adults using the abovementioned framework before and after providing them with a tablet with modern games to play, which enabled them to better express themselves in answering the questions on their preferred content. Preferences regarding this game content and games in general were assessed through questionnaires and a semi-structured interview, in which their experiences were discussed. For more in-depth information, we refer to the article on this study [40].

In Study 2, we investigated the game preferences of older adults in a situation related to the use context of PERSSILAA. Again, the rationale was to give the participant a concrete frame of reference to answer the questions about their preferences. We therefore used a prototype gamified self-management eHealth application that we developed as a research tool: MAGGY. This application combined measuring physical activity through a FitBit step counter with feedback through a mobile gamified activity coach. We refer to the article that discusses the application and the project in which it has been developed in a broader perspective (Tabak et al., unpublished, 2019).

The application measured real-world activity behaviour, step count data, that was compared to a pre-set step goal. The performance of the user was translated into progress and interactions in the game. Participants used the gamified system 
up to three weeks. Demographics, gaming behaviour and game preferences were measured through questionnaires. Perception as well as appreciation of the user regarding the game content presented in the gamified application were measured. Appreciation was measured asking the opinion of the participant on 45 statements discussing examples of game content in the gamified application, perception was measured by asking to what extent the participant agreed to the presented (for example: 'the atmosphere of the game is friendly', 'there is much to explore in the game') (1-5 VAS scale, strongly disagree - strongly agree). Additional semi-structured interviews gave deeper insight into the measured preferences.

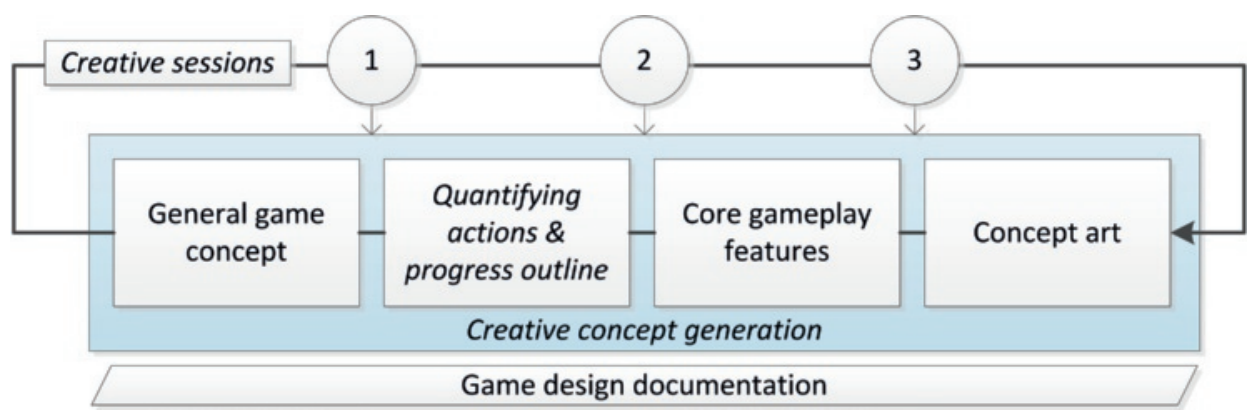

Figure 2 - Game design phase (as applied in case study)

\subsection{Game design process phase 2 - Conceptualisation}

\subsubsection{System and application architecture}

As the non-gamified application development occurs in parallel, its system architecture is analysed to decide on the role and extent of the game-based design within this architecture.

In the case study, the development of the underlying, 'standard' eHealth application occurred in parallel to the four phases of the gamification design process. The system architecture was charted in close cooperation with the back-end developers of the application. In a series of meetings with the project managers and developing partners, decisions were made on the scope of the gamified version in relation to the standard application. Firstly, we decided on the extent of autonomy of the user to choose to use or disable Isections of the) gamified application, and investigated the possibilities to implement these functionalities into both interfaces (gamified and standard). Secondly, we determined the possible variations or restrictions to the functionalities of the application in case specific (negative) health advice is given to end-users. Thirdly, milestones were identified for the inclusion of functionalities from the standard eHealth application in the gamified version at several moments in time. 


\subsubsection{Long-term engagement strategies}

To support and sustain user engagement over a pre-determined amount of time, the components of the standard eHealth application that should be represented in the gamified version for meaningful interaction have to be identified. This is a recommended second step to the conceptualisation phase.

In the case study, we investigated how to quantify the user's performance on the underlying healthcare objectives. This performance needs to be measured as it plays a key role in strategies to sustain the engagement of the user with the application along its entire use time. The activities that enable measuring the progress of the user serve as input for the gamified application and eventually result in game content.

Developing partners in three domains of expertise (physical, cognitive and nutritional health) have contributed to the realisation of functionalities and services of the eHealth application. In conversation with these partners, the functionalities of the standard application that should be featured in the gamified version were specified. To do so, we identified the actions within each of these three domains that were key to the performance of the user as they indicate the training progress of the user. These actions, which we called 'performance indicators', had to be reflected through game content in order to support engagement. We quantified this progress by means of abstract 'game units'. This level of abstraction allowed for the reflection of personal performance, and the possibilities of constructing a system that accommodates for compensation between the progress of different users in order to create a fair and even gaming experience.

Performance indicators were determined for each module through meetings with the partners. To each expertise we asked the following questions:

- Which activities of the user on your module indicate the user's performance?

- Which of those activities have to be reflected in the game layer, to give additional intrinsic value to that activity and stimulate user retention?

- How are these activities measured and quantified?

- What is the distribution in game units (GU) you consider optimal?

In addition, we discussed possible ways to misuse the service that may result in undesired negative health outcomes, to counter misuse.

\subsection{Game design process phase 3 - Creative design}

In the previous phase, the elements of the standard application that should be reflected in the gamified version were identified. In this phase, these elements are concretised into the design of a meaningful user experience, including aspects 


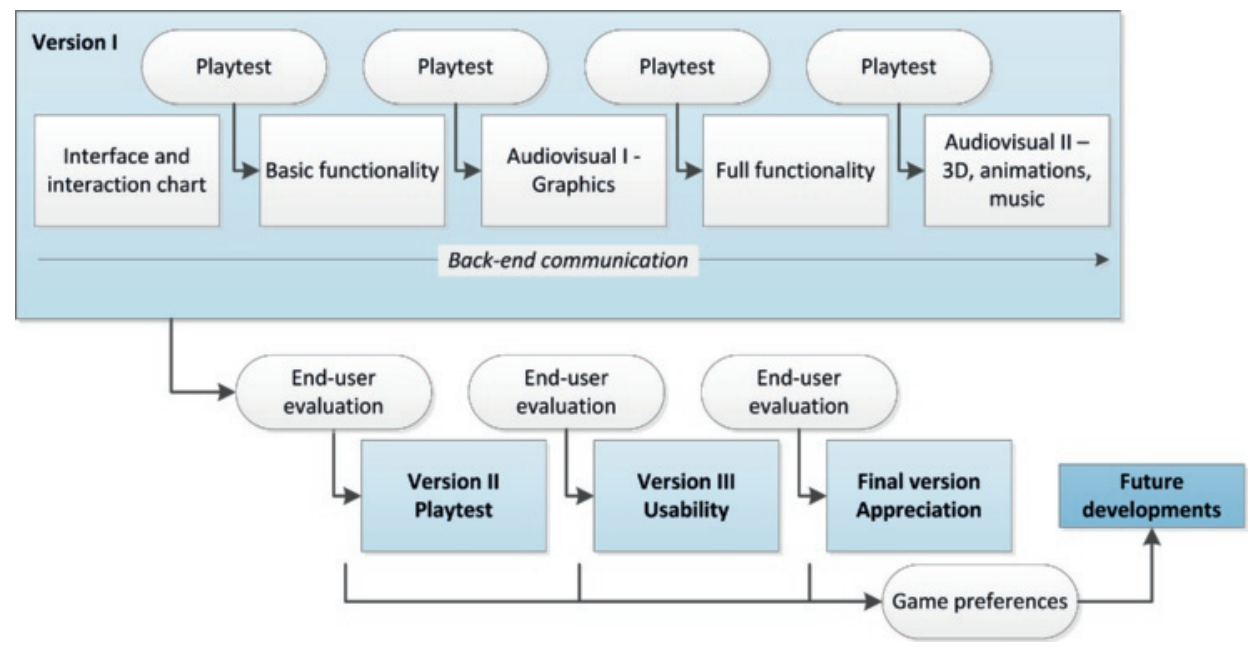

Figure 3 - Refinement phase (as applied in case study)

such as gameplay and storyline, utilising the knowledge on suitable game content gathered in phase 1. The result of this phase is the creative concept of the gamified application, often referred to as the 'high concept'.

We subdivided creative design into three key topics: 1) the general game concept, 21 core gameplay features and 3) concept art, covering the visual and auditory outline of the game. We approached the game design phase as a cyclical idea generation process supported by creative sessions (fig. 2). The game design itself occurs within the creative concept generation. In addition, brainstorm sessions serve to reflect on and generate new insights and ideas. Quantifying performance indicators and outlining the game progress are addressed separately. During this phase, the game design documentation is created [4 1 ]: a living document, accessible by all members of the developing team, that is continuously edited and updated. The document is a blueprint of the gamified application and served as a communication tool between all parties involved, and supports organising development activities. The document covers all aspects important to the development of a game, describing the vision, contents and planning stages of prototyping and implementation, as well as any outsourcing plans.

In the case study, three larger sessions have taken place during the creative design phase to support the development of the creative concept by the game designer, and to ensure optimal integration of all stakeholder's interests.

The first session included all developing partners of the PERSSILAA project. Participants were introduced to gamification through a presentation on the intention and rationale of the use of gamification for PERSSILAA, and the results of the previous phases in the game design process. With this background, 
the creative concept for the gamified application was discussed and ideas for the game concept were generated. Topics of these discussions were desired functionalities and interactions, user-generated content and sharing, as well as (graphic) design and themes in general.

In the second session, participants with a professional background in gaming, back-end developers and the PERSSILAA project manager were included in a group ideation. In this session, ideas on specific game content and mechanics were generated through discussion and pen and paper techniques such as mind mapping. The topics included feedback- and reward systems, core gameplay elements such as discovery, collecting items and level progression, as well as ideas for the central theme. Also, opportunities for communication flows between users, or user and game, were presented and discussed. Furthermore, concepts for the interface design and planning for technical integration were prepared.

In the third session, developing partners in the three domains (physical, cognitive and nutritional health) participated. A presentation was held on the progress of the project and developments related to underlying research and game design developments. Then, by means of ten propositions, the suitability of specific game elements was questioned based on insights of participants within their own expertise and experience with the target group, research and related technologies. Topics of these propositions included the gamified representation of performance through the game, the creation of a multiplayer mode, possible pressure posed on the end user by the game to perform the exercises and the suitability of the reward systems used, i.e. progress in the game opposed to points or virtual currency.

\subsection{Game design phase 4 - Refinement}

The last phase of the gamification design process covers the development of the prototype. This occurs in several stages, aided by a series of evaluation sessions. In figure 3 we illustrate this phase, as applied in the case study, in simplified form along its major milestones.

\subsubsection{Prototyping}

As the fidelity of the prototype evolves from low to high, the back-end, functionality and visual features mature until the fully functional prototype is created. In early development, refinement iterations are more rapid and substantial.

In the case study, while prototype development has been a continuous process, four main versions were created as predetermined by milestones set in the conceptualisation phase. Version I encompassed realisation of the game that was increasingly functional as well as graphically attractive. It included the 
development of the game interface and interaction design on paper to the basic functionality with improvised graphics, to a more and more visually polished version. Within the development of this first version, the back-end was increasingly linked to the frontend (i.e. the game). The developing team and researchers thoroughly tested ('playtesting') each iteration of the first prototype to evaluate game design and usability, eliminate bugs and design flaws in an early stage. This first version of the game was then used in an evaluation session with the end user.

\subsubsection{Evaluations}

In the case study, three end-user evaluation sessions with specific focus have taken place. Study results from phase 1 suggested that end-user involvement would be most valuable after the creation of the first functional prototype (Version I) and onward. Game preferences and usability requirements of the target group were thoroughly investigated beforehand. Also, the results led to new insight that these game preferences strongly diverged from the characteristics of participants' current favourite games, possibly influenced by their (lack of) prior experience with, or access to, modern video games.

All sessions delivered the following input for the succeeding version of the prototype: 1) information on the end user regarding demographics, current gaming behaviour and past experience with games and devices, 2) perception and appreciation of the game content that is present in the game and 3) information on technology acceptance, perceived motivation and usability. The data on game preferences was gathered to follow-up our research as presented in phase 1, in order to refine and expand the user profile of the older adult. The domains discord, dedication, novelty, social, intensity, and threat apply to this game concept and are used to describe the preferences of the user for specific game content on a linear scale.

\section{Session 1}

The first sessions focused on playtesting the gamified application with the end user. The aim was to gather feedback on the game concept, playability, appreciation of the diverse game mechanics and the expected added value of the use of the game over the use of the standard application. Participants were recruited by Roessingh Research and Development (RRD, scientific research institute for rehabilitation and chronic care, www. rrd. nl) for inclusion in PERSSILAA and informed in an information meeting. In this presentation participants were instructed on the use of both versions of the application. An exclusion criterion applied in case of health issues limiting the use of the original application's training modules. This criterion was automatically fulfilled, as participants of the standard application were previously assessed and classified as pre-frail. 
Participants used the application, basic or gamified, in their home environments. Participants were asked to log their experience with the game and to keep track of any issues that might occur. For the purpose of troubleshooting and tracking game use, log data was gathered. After a use time of 6 weeks, participants received an invitation via e-mail to fill in an online questionnaire on their experiences. An option was included to elaborate the choice to not use the game as well. In this questionnaire, demographics and information on gaming behaviour and experience with devices and games were gathered. The questionnaire included questions on the theme of the game, the gameplay, the graphical style and the used game mechanics such as the game progress. Furthermore, questions were asked on the clarity and use experience of the gamified interface compared to the basic application. These aspects were measured in 34 items rated with a 5-point Likert scale. Game preference (perception and appreciation) was measured by means of two times 17 statements on content present in the game.

\section{Session 2}

In the second session we evaluated the game concept, the expected use and motivation by the game on the long term. We measured the perception and appreciation of the presented game content by the user. This session focused on improving the usability of the game by means of video recordings and a the 'thinking aloud' method.

Participants were aged 65-75 years. Recruitment was done by means of an information letter, spread by Twentse Zorgacademie (TZA, testing and training centre for care technology, www.twentsezorgacademie.nl), and the test location was set at TZA Living Lab, Enschede. Participants were excluded from the study when they had no experience with using a pc, or when they were not interested in the use of the technology used in PERSSILAA. As the (physical) exercises from the PERSSILAA application were not part of the study, a lesser physical condition of the participant was not considered an exclusion criterion.

People participated in pairs. Participants received an introductory presentation by the researcher, informing them on the use and goals of the PERSSILAA application and game, as well as on the study set-up and aims of the research. Participants were invited to access the game and to perform specific tasks or actions (divided over sets of five sessions to avoid knowledge saturation [42]), while speaking their minds and discussing with each other. The underlying exercises as offered by the PERSSILAA application were simulated, to enable a walkthrough of the entire game in approximately an hour. Using screen capturing software, actions and speech of the participants was recorded. After the session, participants were invited to fill in a questionnaire in a second room on (separate) pc's. 


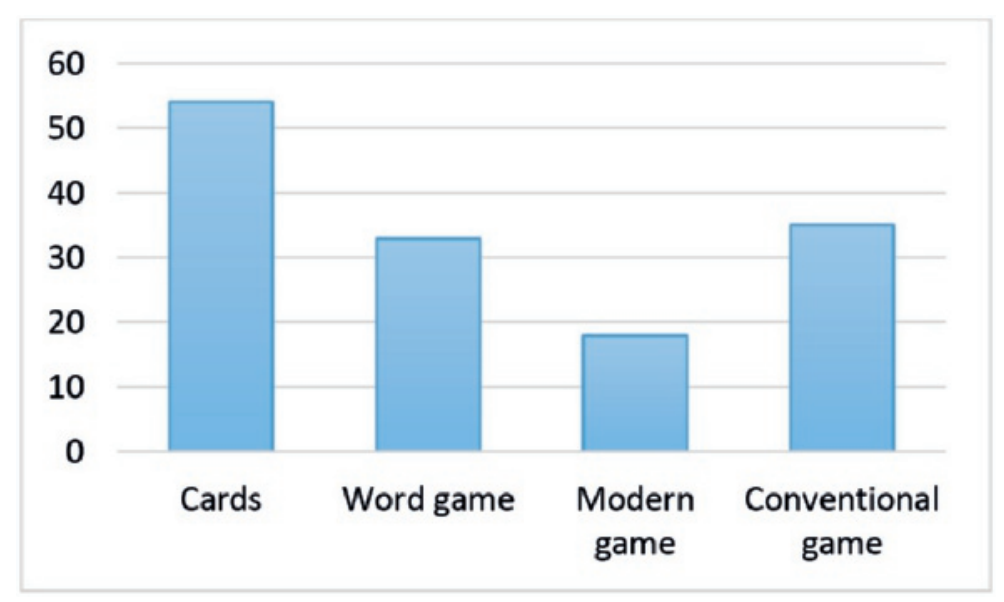

\section{Figure 4-Participants' current favourite games}

The questionnaire assessed demographics and information on current and past gaming behaviour and experience with the use of devices of the end user. We measured the opinion of the participant using the UTAUT questionnaire [43] for performance and effort expectancy (76 items rated on a 7-point VAS scale). Formulation of the questions was adapted from the original to the technology used in this particular situation. The expected use of the game was measured by presenting a hypothetical use situation. In addition to the video and audio recordings, the questionnaire assessed usability of the game in general and of specific aspects of the game. Scores on these aspects (overall appreciation, ease of use, performance and effort expectancy including expected effectivity versus motivation from the game, fun factor and intended future use) were calculated. The preferences for game content of the end user were measured by means of two times 51 propositions on the content present in the game (5-point Likert scales).

\section{Session 3}

The third and last evaluation session used the same methods of session 2 . However, the game was presented to the user in a stand-alone version that could be used from the participant's home situation and was not played in couples but individually. No audio or video recordings were made. The questionnaire was filled in through an online form. 


\section{3| Results}

\subsection{Phase 1 - End-user research}

\subsubsection{Characteristics and limitations}

The target group is the older adult, aged 65-75, with sufficient computer literacy to independently use mobile devices or a pc and an interest in digital games. Of the older adults questioned ( $n=136$, mean age 69 ), $75 \%$ indicates to play games of which $75 \%$ plays at least once a week or even daily (more than $40 \%$ in total). Approximately $75 \%$ of frequent players indicates to play digital games (or $42 \%$ of all participants), next to board games or other conventional games. Computer and laptop (76\%) and smart devices (60\%) are the most popular media for playing. Two thirds of the total number of participants (including solely board and/or card game players) choose playing together (cooperative or competitive) above playing alone. Only few participants indicate $(n=12)$ that they play online social games, of which Wordfeud is mostly mentioned, or are interested in doing so in the near future. The older adult prefers to play games at home rather than elsewhere or on the go (90\%). Participants' favourite digital games (fig. 4) where thematically categorised into card games (e.g. Solitaire, FreeCell, Spider), word games (Wordfeud, Ruzzle, crosswords), digital versions of other conventional games (mahiong, chess, cryptograms, quizzes, bingo) and modern (online) games (candy crush, bubble shooter, search and find games). Interest in more modern games is relatively small, $13 \%$ of all answers, but present. Participants play digital versions of conventional games, of which relevant examples are Scrabble, Rummikub, a diversity of card games and puzzle games are mentioned most often. Of all participants, $74 \%$ indicated to enjoy trying out games they do not know. In interviews, many participants find modern games suitable only for younger generations or their grandchildren, but not for them.

\subsubsection{Game preferences}

The preferences of the older adult after playing new games or using a gamified application through questionnaires were assessed. Results of Study 1 were presented earlier in De Vette et al., 2018b. In Study 2, the scores on perception and appreciation of game content were mapped onto the classification, resulting in a user profile (fig. 5). The scores for the user's perception on the game content are shown in coloured, taller bars. This is the 'game profile', from the viewpoint of the older adult. The shorter, hatched bars indicate the user's appreciation of this game content. Each domain is described by two extremes, for example Intensity is described by content that ranges from slow-paced to exciting.

The profile illustrates the following findings. From the overlap in scores of the content of the game according to participants ('perception') and the preferred game content for a satisfying gaming experience ('appreciation'), we notice that for a number 


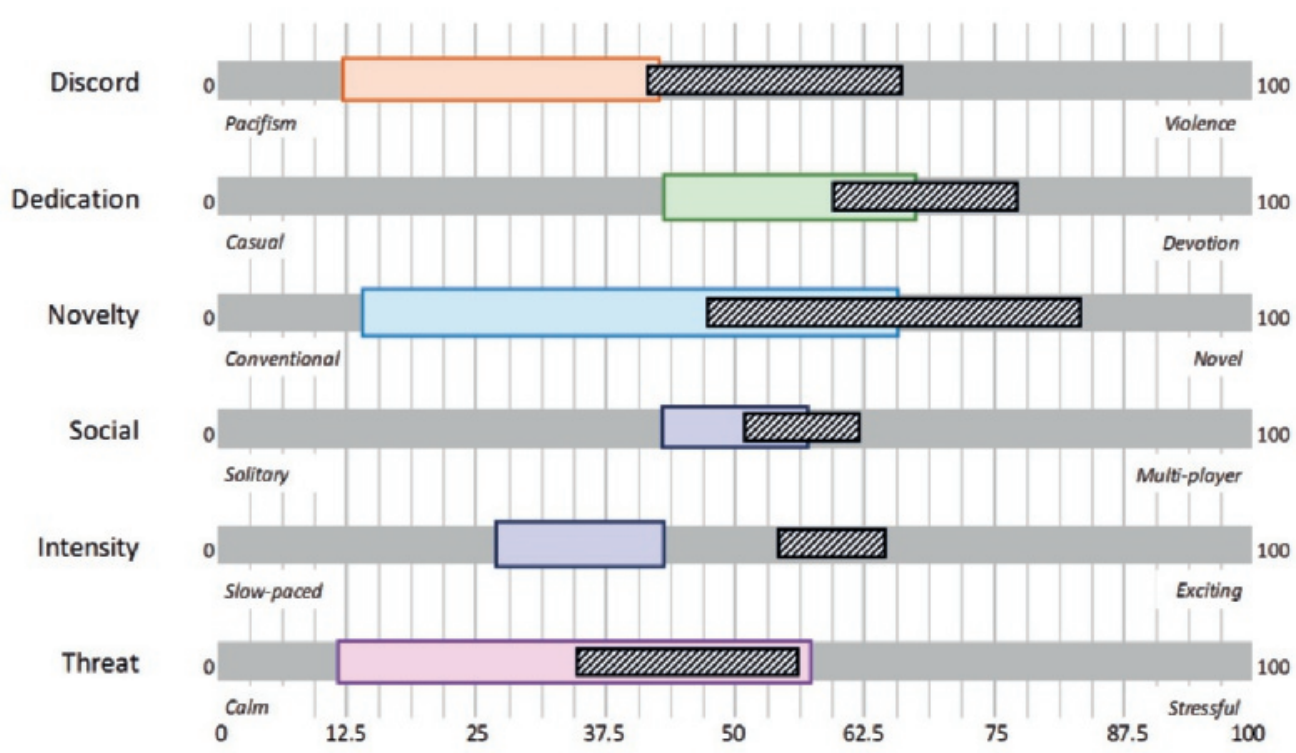

Figure 5 - User profile (perception: taller solid bars, appreciation: shorter hatched bars)

of participants, the game is insufficiently competitive and not demanding enough devotion. This includes challenge, effort needed to play and learning involved. The game was considered conventional to moderately novel, including variation and aesthetics, which may not have been sufficient to engage the user. Participants rated the social component present in the game as neutral, as both solo and multiplayer options were integrated, while slightly preferring a multi-player over a solitary game. The game was not sufficiently exciting for the participant, intensity of the game was rated below preferred. The contents of the game on the domain of threat were appreciated as they were, from which we conclude that the game was not too stressful or frustrating. As discord (violence) was no apparent game feature, the scores on this domain remains inconclusive at this point for use in PERSSILAA.

From the additional interviews (for which we refer to the original article) we observed the following, that gives deeper insight in the abovementioned scores on the classification. Firstly, most strikingly, participants were open to modern games and preferred less conventional game content than indicated in the earlier questionnaires. One of the games from study 1, Monument Valley, was particularly appreciated and played for many hours, even by those who were sceptical about (modern) video games on beforehand. Participants indicated that playing had changed their attitude towards modern video games. Moreover, in study 2, the game concept of the eHealth application was not appreciated at all. The game was considered not novel enough, and the conventional game concept of crosswords was not 
found attractive. The game did not have enough variation to maintain engagement. Participants indicated the game was too similar to the games they already knew, and the gamified application therefore did not fulfil a need.

In the off-the-shelf games and the gamified application, problem solving, intellect and thinking were found particularly motivating. The puzzle and brain training aspect of the gamified application was appreciated by most users, despite being redundant among their current gaming behaviour. Aesthetics in the game, such as good graphics and attractive artwork, were appreciated. Other motivating game characteristics related to novelty are variation, curiosity and discovery. Participants indicated that they thought a larger selection of games would contribute to their motivation to use the gamified application.

While the older adult has an interest in social gameplay in conventional games (board games, cards), they indicated that digital games are mostly enjoyed alone. In the interviews from study 1 and study 2, after the use of games or a gamified application, participants indicated that they found it enjoyable to withdraw and relax with a videogame on their own. The participants indicate that they are competitive, but this aspect is not looked for in social contact through games. In the gamified application, two social playing modes were included (competitive and cooperative), but neither options were used by the participants. Participants indicated that they did not feel the need to share gameplay, the progression in the game or their activity behaviour with other people.

We observed that the older adult has a low tolerance for frustration and negativism. Game content that creates feelings of unfairness or lack of control should be avoided. Offering additional hints, cheats or help may reduce feelings of frustration or incompetence according to the user. Participants indicated to feel underestimated by presenting game content that is superficial and easy. A childish or silly theme would however not be appreciated either. Participants indicated that a fast-paced gameplay, demanding a too high level of physical agility or reaction speed, was disliked in general. All participants indicated their strong aversion to violence.

Participants enjoyed being challenged or to challenge themselves, achieve goals, progression and development of skills. Participants made use of statistics in the gamified application to challenge and improve themselves. Clear goals and trackable progression were mentioned as contributing to a positive experience in both studies. In the gamified application, the link between real world activity and gameplay was not always clear, which was found demotivating. Trial-and-error in general is not an approach most older adults wish to take in games. Participants indicated that it is important that games can be paused and continued at any point, and that controls that are simple and intuitive and a goal that is clear at all times contribute to a positive experience. 


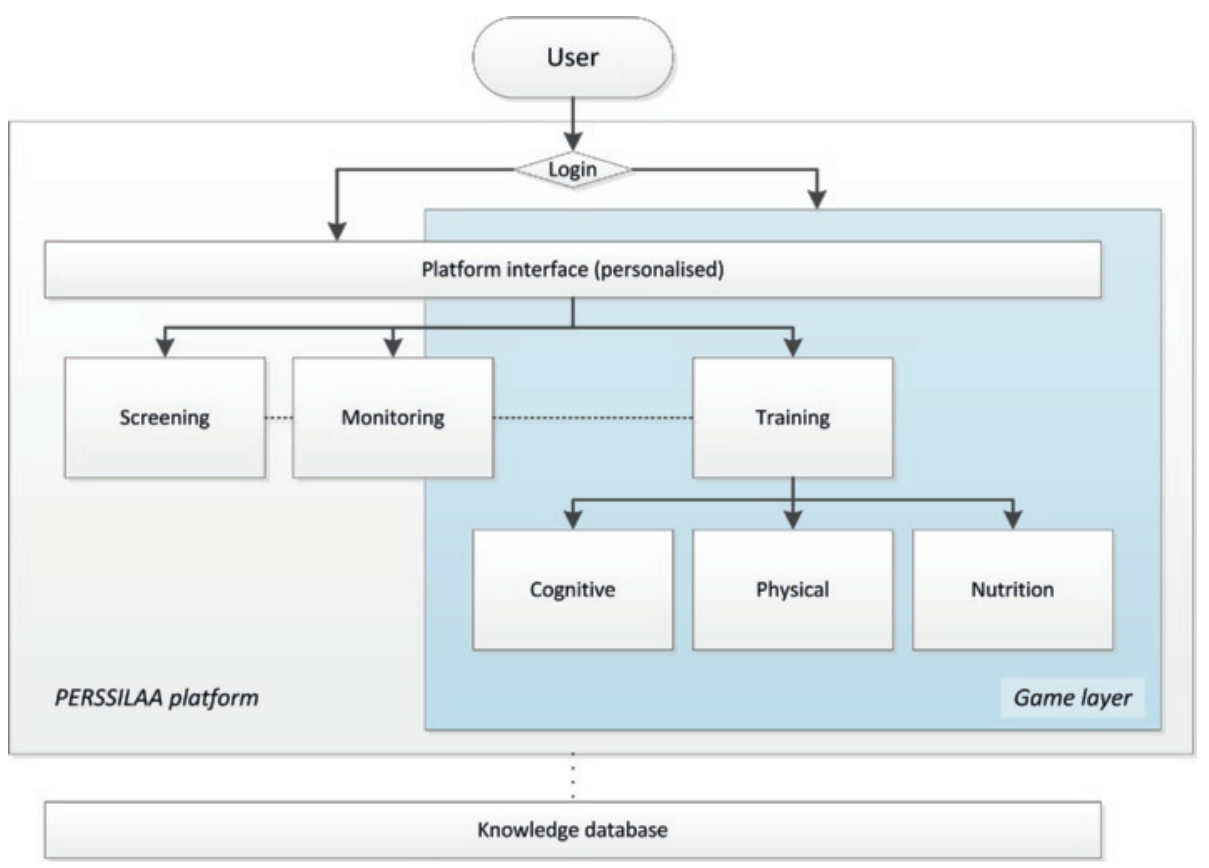

Figure 6 - The PERSSILAA system for the older adult end user

\subsubsection{Recommendations for engaging game content}

From the abovementioned results, we deduced a set of guidelines for game design for the older adult. We aim to create a game that include the following characteristics:

- Design for moderate to high Novelty

- Novel game concept, offering variation, renewable content, enabling exploration and triggering curiosity

- Attractive aesthetics and artwork, storyline, graphics

- Focused on problem solving, logical reasoning, thinking and puzzle

- Provide clear rules and objectives

- Design for moderate to high Dedication

- Create challenging gameplay, enabling achievement, learning and mastery

- Offer content for devoted gameplay, such as challenges and objectives with increasing difficulty

- High dedication should not be compulsory to play the game at all times; add free, casual gameplay elements and low difficulty levels as well for balance

- Design for low Discord and Threat

- Neutral content, relaxed and cheerful atmosphere, not violent or overly friendly or cute 
- Avoiding content that triggers negative emotions such as stress, tension, a disturbing setting, frustration, anxiety and unfairness

- Remove any possibility to fail, avoid negative feedback

- Design for moderate Intensity

- Suitable game intensity is moderately paced, avoiding exerting game elements

- Demanding focus of the user to complete objectives should be moderate or alternating, for example multi-tasking at high speed should be avoided

- Design for low Social

- As our results did not strongly indicate the added value of a social game component, the initial version of PERSSILAA will be focused on the solo player

While we take into account the available usability requirements for (game) interface design for the older adult [44-48], we regard the following game-related usability specifications:

- Goals must always be clear and progress trackable, as well as the link with those of the underlying healthcare application

- Complex movements and controls should be avoided, game controls should be basic and intuitive

- Completing game objectives may never relate solely on the agility of the player

- The game in itself should be accessible, simple game mechanisms are preferable over more complex ones

\subsection{Phase 2 - Conceptualisation}

\subsubsection{System and application architecture}

The result of the eHealth application development is the PERSSILAA platform. This web-based service is accessible to several users: the older adult or patient, the caregiver and the healthcare professional. The back-end (knowledge database) is accessible to the system administrator (at RRD, Enschede) and researchers. The focus of this article lies on the service for the older adult. The online self-management platform offers a monitoring and training program that supports both acquiring and maintaining a healthy lifestyle. The platform is particularly intended for people in a so-called pre-frail state, who show first signs of decline but are not yet in need of professional care. People with proper levels of functioning are however also encouraged to use the service, as training can prevent or delay becoming vulnerable for age related health decline.

The functionalities of the platform for the older adult are visualised in fig. 6. Firstly, the user logs in and is given the autonomy to choose at all times between the 
'standard' and 'game-based' version of the platform. From within the game, the personalised (non-gamified) platform interface, or "start screen" can be recalled. The functionalities in the standard PERSSILAA interface are data-gathering questionnaires (screening), data on interaction with monitoring devices (monitoring) and training modules (training). Training contains three modules, which are interconnected and are centred around physical, cognitive and nutritional well-being. The collection of data that occurs through the screening process module, via questionnaires, and the network of sensing devices forming the monitoring module, such as a step counter and digital scale, is out of scope for this article. The functionality of the game-based version is limited to the training modules and a representation of selected monitoring data.

Milestones were set for the development of the game-based version over time, to determine its functionalities to be implemented for each following version from a first prototype to future versions. We define four versions with increasing added functionality of the standard version into the game-based version:

1 Prototype, due at the end of refinement phase (phase 4 of gamified design process). Functionalities from the original application to include for the development of the prototype: training modules, basic monitoring.

2 First follow-up, due at the end of the implementation phase. Functionalities are those of the full platform: training, monitoring, screening.

3 Second follow-up, due in the maintenance phase. Planned functionalities for the eHealth application that should be part of the gamified version in the future (wearables, smart devices, digitising of the intake process). Extension of game design such as an interactive social platform, extension of already implemented functions.

4 Future versions: opportunities for future game design functionalities, unrelated to or derived from the underlying application.

The development of the initial version of the game-based application is described in this article, as visualised in fig. 1. In this stage, the platform has fully accessible training modules as well as a small part of monitoring feedback through the game layer. At the login screen the user can disable or enable the game version of PERSSILAA at all times. This selection does not influence the contents or progress on any of the modules.

\subsubsection{Long-term engagement strategy}

Interviews with developing partners resulted in 1 / a list of activities for each particular healthcare domain that were identified as relevant indicators of user performance and 2) the assignment of fictive game units to the identified performance indicators. An excerpt of the performance indicators and their respective game units for the physical module is shown in table 1. The physical module provides exercising for 
Table 1 - Performance indicators of physical training

\begin{tabular}{|l|l|l|l|}
\hline Warming up (max. +6 GU) & 4 exercises & $\begin{array}{l}\text { Commencing } \\
\text { Per finished exercise }\end{array}$ & $\begin{array}{l}+1 \mathrm{GU} \\
+1 \mathrm{GU}\end{array}$ \\
\hline Training session (max. + 1 1 GU) & 9 exercises & $\begin{array}{l}\text { Per finished exercise } \\
\text { Finishing training (limit: } \\
5 \text { skipped exercises) }\end{array}$ & $+1 \mathrm{GU}$ \\
$+2 \mathrm{GU}$ \\
\hline Cooling down (max. +6 GU) & 4 exercises & $\begin{array}{l}\text { Commencing } \\
\text { Per finished exercise }\end{array}$ & $\begin{array}{l}+1 \mathrm{GU} \\
+1 \mathrm{GU} \\
+1 \mathrm{GU}\end{array}$ \\
\hline Full session & Finishing & $+5 \mathrm{GU}$ \\
\hline Answering questions & & & $+1 \mathrm{GU}$ \\
\hline Regularity of training & e.g. 3x weekly & & $+5 \mathrm{GU}$ * (1,05/week) \\
\hline
\end{tabular}

strength, endurance and mobility, using diverse monitoring and feedback methods on daily activity patterns. For brevity of this article, we leave the full list out of scope and refrain to briefly discussing the implementation of the other two training modules and the screening and the monitoring modules in the prototype.

The interviews resulted in notes of caution regarding possible misuse. Encouraging practice while experiencing pain, for example, must at all times be avoided. Skipping exercises or terminating the training must not be discouraged, and a limit of skipped exercises in order to complete a session may be set. Stimulating the user to practice exercises that are below their current performance level, to generate quick rewards in the game, should be avoided. Cheating the system just to gain access to new game content will be prevented as much as possible but cannot be avoided in each case. We believe in the good-will of the user and his or her motivation to improve, and intend gamification to reinforce this motivation for the exercises provided.

\subsection{Phase 3 - Game design \\ 3.3.1 General game concept}

The general game concept is a gaming 'environment' that is interactive, scalable and expandable. To the user, this gaming environment is an alternative interface that, if desired, fully replaces the original application. The gaming environment is scalable in terms of adaptability to fit the user-specific needs for access and restrictions to underlying platform functionalities. It is built to for expansion with new game content to extend the time that the game is interesting to play, in such a way that changes to the original platform do not impair the functionality of the game even during use by the end user. The game concept reflects these demands through its interface and game progress design, which is driven by interaction with the underlying training modules. 
Figure 7-Mindmap

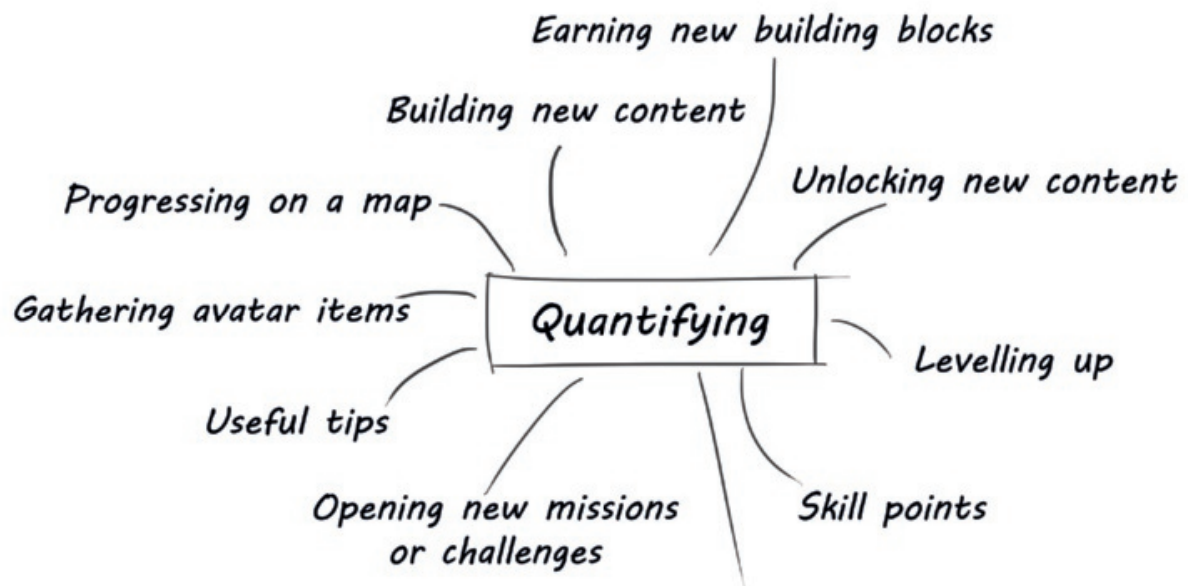

ELO-system

Following the results of Phase 1, various gaming concepts were explored. A theme was created that allows for offering sufficient variation and exploration for the user, which is recognisable and can be given clear rules and boundaries at the same time, and can be combined with an aspect of logical reasoning and puzzle. The chosen concept is a map based and story-driven game, to stimulate intrinsic motivation driven by curiosity and achievement. Diverse elements are added to enable various levels of achievement, from casual to more devoted gameplay. Ideas that emerged from the sessions were mini-games on different map locations, including activities related to the themes of the modules, and a showcase of trophy items to gather over time.

\subsubsection{Quantifying performance}

From the general game concept, a suitable representation in the game of the performance of the user on the underlying training modules was created. Figure 7 shows one of the brainstorm notes from the sessions on possible solutions for the representation of performance that, through the game units, must receive a suitable, unobtrusive antagonist in the game environment. The brainstorm was aimed to avoid the obvious, extrinsically rewarding options such as game currency and simple point systems, and think of intrinsically motivating options instead.

The storyline in the game is the main mechanism to provide feedback and motivation on the activities on the training modules. It was planned to integrate game content for a total playing time span of 12 weeks, as this reflects an optimal use time of the 
original application's training and monitoring goals. Through user performance, the environment evolves and new areas to explore unlock. These areas contain gameful activities (e.g. mini-games) that can be played freely, which is again rewarded with a part of the storyline of the game. The storyline should complete in approximately 12 weeks, and from there can be extended with cycles of the same setup but with different content.

\subsubsection{Core gameplay features}

The game was titled Stranded! (translated from Dutch 'Aangespoeld!'). The highlevel narrative is that the player is an adventurer in late Victorian times who is shipwrecked on an unknown island in a storm. The parts of the boat that have been scattered all over the island have to be retrieved in order to build a new boat to get home, before the volcano erupts. Every next level is opened after passing a personalised threshold of performance on the platform defined by game units. From completing each level, that will have a puzzle character, a boat part can be gained. Eventually, when the user has completed the boat, a next island can be made available to explore (fig. 8).

The game environment is built up from a number of scenes. Major locations are:

- An overview screen, or map, of the first island (birds-eye view)

- A two-part landing page or home-screen which (like the portal) gives access to the modules as well as the pier where the lifeboat is constructed

- 12 in-level screens such as mini-games and representations of the modules for monitoring and nutritional advice

When the game is started for the first time, an opening animation will show that introduces the backstory. Upon finishing the first cycle of the game, an ending animation is shown as well. The player starts at the home-screen in each new session. From here, the training modules can be accessed directly through small wooden cabins. The scalable aspect of the game layer is realised through these wooden cabins, that can be added or removed to suit the training advice of the end user. Four cabins will be displayed in the prototype which can be set to opened or closed. The screen pans if the player walks to the left of the beach, showing another cabin that is connected to the monitoring module as well as the boat pier. From the beach, the player can move to the island in birds eye view. This map of the island will in time reveal new locations. In these locations, mini-games with each their own levels and graphic concept can be played. These games can be revisited as desired. The first version of the game layer is expandable with more map areas and levels in mini-games. The monitoring layer interacts with the game environment through the arrival of messages in a bottle which contain items that can be stored in a trophy hut, reflecting the overall timeline and monitoring achievements of the user. For the initial prototype, the storyline and interactive surrounding is kept to a minimum. Functionalities that have been designed to include in later versions (e.g. during 


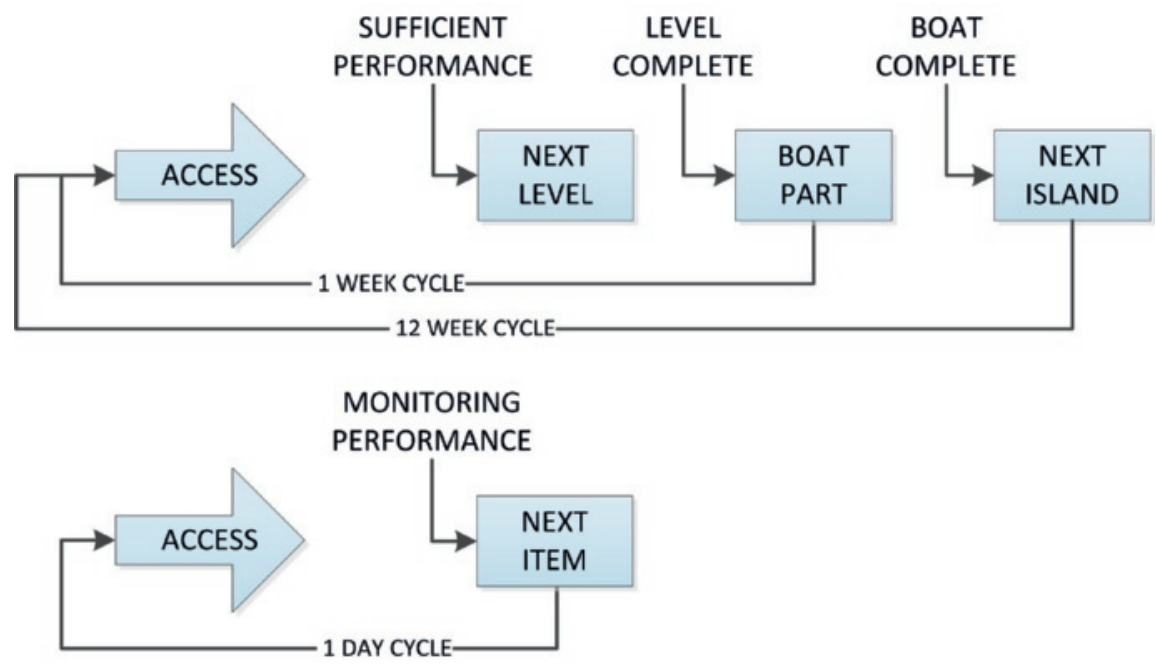

the maintenance phase) are an animated map that shows the volcano's activity, an interactive boat building site that requires boat parts but also the gathering of necessary tools and creative solutions for parts that are broken beyond repair and the sourcing of food for energy to proceed. Some food can be found in the game, but the rest must be earned from following the training modules. A social component for a future version could be added by adding the function of taking snapshots of scenes and environments that can be share with others. The cycle of 12 weeks can be prolonged by extending the storyline to a second main location or island.

\subsubsection{Concept art}

Concept art covers the visual and auditory outline of the game, including graphics, animations and sound design. Results in this section are style mood boards and interface mock-ups, graphic level (mini-game) themes, character drawings, animation storyboards, game music compositions and sound effects. A summary of concept development is given in Multimedia Appendix 1. Intro and outro animations can be seen in Multimedia Appendix 2.

\subsection{Phase 4 - Refinement}

\subsubsection{Prototypes}

The final prototype was created through an iterative prototyping and evaluation process. The initial version is the result of the research described in Phase 1 as well as rigorous playtesting with the developing team and researchers. These playtests focused on connectivity and functionality of the game layer. In each playtest, the full game was played and all functionality checked. The functionality of the game was 
constructed and tested using simple visuals, that are exchanged for mock-up images and, when finished, the final graphic design (fig. 9). Multimedia appendix 3 shows screenshots of these development cycles. Three more evaluation sessions, with end users, led to the refined final version of the prototype. Throughout, improvements on gameplay and navigation were made, as well as intuitiveness of the user experience design. An information point providing short explanations on items and functionalities on screen (i-icon in the corner of each screen) was created. Lastly, information was gathered on refining future versions (in the maintenance phase) as well as preliminary implementations for future functionalities.

The main character that was developed is a female explorer named Sophie: a likeable character that fits the storyline well, rather than a character that resembles the player itself. We created a 3D animated character, which demanded a larger initial effort but creates more possibilities that are eventually time-saving over the use of $2 \mathrm{D}$ animation. In the opening animation we see Sophie on board of a ship that is caught in a storm. She falls over board when a sudden wave tilts the ship, and she washes up on the beach of a seemingly desolate island. In the initial version, the animation is based on sketches. The final version holds two 3D animations (intro and outro, introducing a follow-up story) including sound effects.

Every session starts with the choice for a classic or a gamified portal using a slider. The application will remember the choice, but it will always appear at login. The player starts on the right side of a panning scenery showing a beach. From here, the player can start exploring the area and access huts which are directly connected to the training modules. The user can access a virtual crop field and cooking area ('moestuin', to be paired to nutritional advice), go to the rest of the island to explore mini-games ('naar het eiland') or to the left side of the beach where the boat is built ('boot'). Items that wash up on the beach in bottles and some interactive surprises (or 'easter eggs') which are crabs were created. These crabs seem to be non-interactive, but after some trying it appears that they can be caught. These bottles are the main feedback from the monitoring module. Some contain seeds that are earned from training and through monitoring sufficient exercise which can be planted and grown in the crop field. Once harvested, they can be used to cook meals. The left hut is opened and leads to the physical training module. The other huts are constructed to be coupled with the nutritional and cognitive modules. The island map displays levels that can be played, which have been opened by means of completing training or exercises. Six levels have been created for the final prototype, all complete with visuals, music (can be switched off/on) and sound effects. After finishing each level, e.g. the first objective of each mini-game, a piece of the lifeboat will be added to the construction. The image shows the progress after approximately two weeks of practicing and playing. The image below depicts the fully completed boats after twelve weeks. 


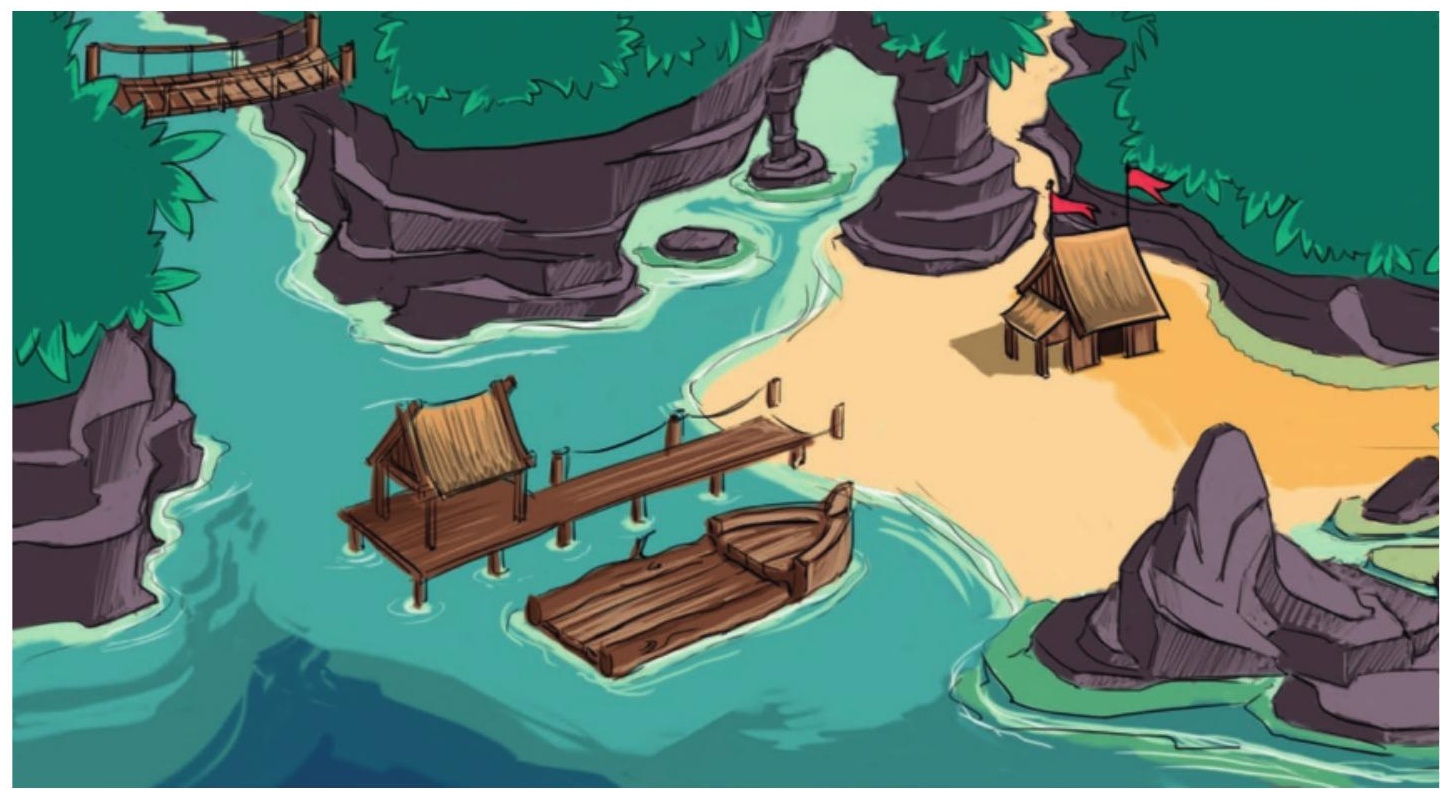

Figure 9 - Final graphic design home-screen

\subsubsection{Evaluations}

Results of each of the evaluation rounds are summarised in table 2. From the log files of the first evaluation round was observed that 9 persons explored the game without playing actively before returning to the use of the standard platform. Among reasons given were computer issues $(n=1)$, looking too complicated $(n=1)$ and distracting from the original exercises $(n=1)$. Two participants indicated to have forgotten about the game, and that they would like to get another chance. The game was played in combination with the underlying physical activity training module by 4 participants ( 2 male, 2 female) for a longer amount of time ( 1 to 6 weeks, one person weekly and three persons daily). Of these participants, 3 were frequent players. The appreciation for the game ranged from mildly to extremely enthusiastic. Two people indicated they were highly motivated to do the exercises because of the game. None of the participants were interested in playing the game together or sharing their progress. In the game, the storyline and the overall gameplay were the factors that appealed most to the participants, while the in-game explanations were found too limited. Two participants found the level of challenge in the mini-games just right, one found it too difficult and one too easy. The controls of the game were not found sufficiently intuitive to use.

Using this information, pop-ups with short instructions or explanations on the current section of the game, which can be recalled at all times by clicking on the info icon in the corner of every screen, were implemented. Also, the mini-games were given a tutorial through a very easily solvable first level with detailed explanation. The 


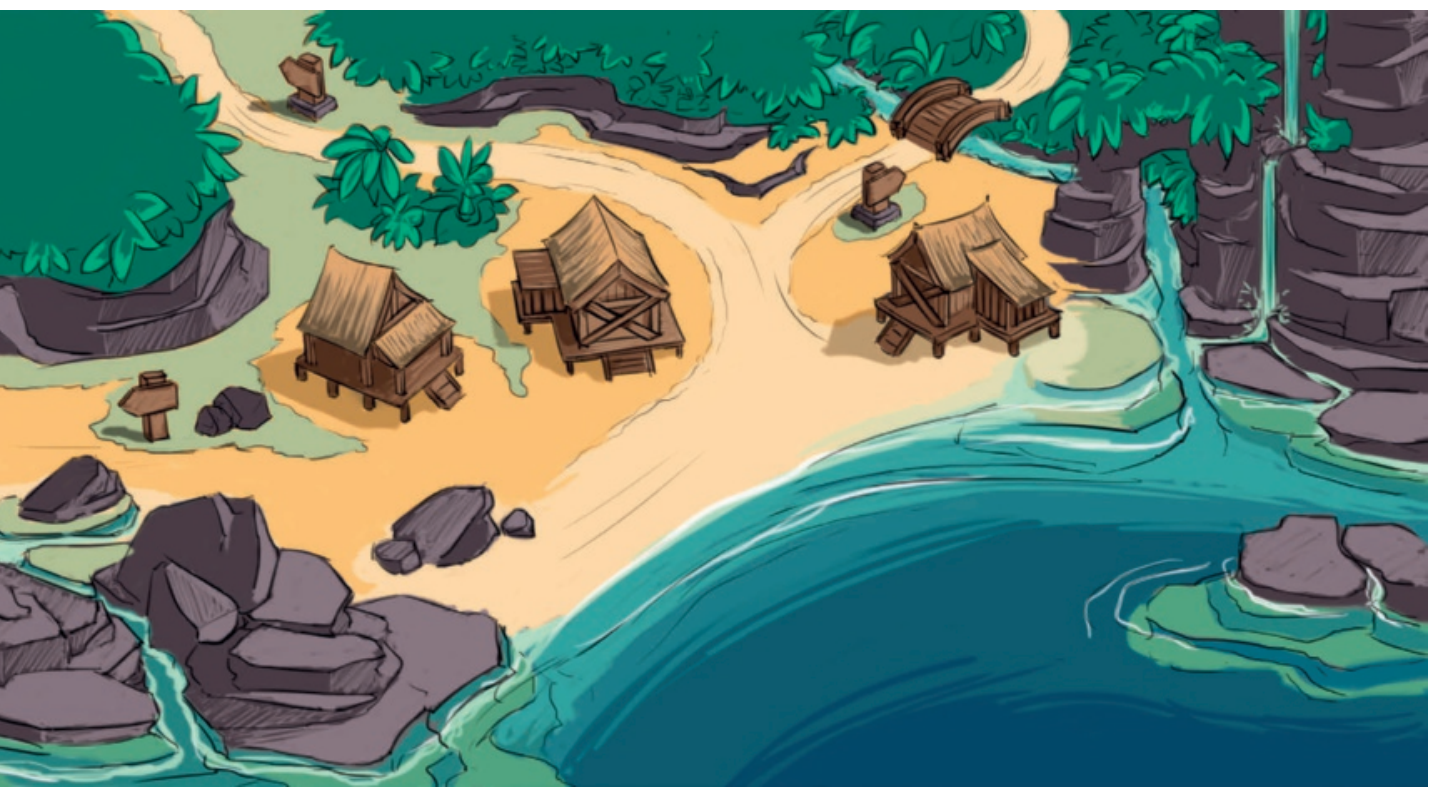

controls of the main character were revised to make them easier to understand and activate, by reducing moving around to just one click or tap on the screen.

In the second round of sessions, the game was played from beginning to end using instructions of the researcher using simulated exercises to drive the game. Participants indicated that the mini-games $(n=5)$ and gathering boat parts $(n=4)$ were the game's best features. The gamified platform (including the exercises) would be recommended to peers by 7 participants, and 8 would like to play the game again in the future. From the analyses of video (thinking aloud method) and screen captures, we observed that the explanatory text of the mini-games was skipped by the majority of participants. One of the mini-games controls were not understood at all because of this. Participants said they did not want to take time to read the texts, or thought they would understand the games without the explanation. None of the participants returned to this information afterwards, but were inclined to give up without trying.

After this round of sessions, minor usability issues in the game were solved and the audio was improved on lag and volume. Although the crop field was not enjoyed much, plant growth speed and feedback on progress were improved. The minigames had several unclear aspects that appeared to be caused by the explanations not being read. Therefore the introduction to the (new) mini-games was improved, made shorter and more clear using images. The one mini-game that could not be played by the majority of participants was fully replaced by another game. 
Table 2 - Results of evaluation studies

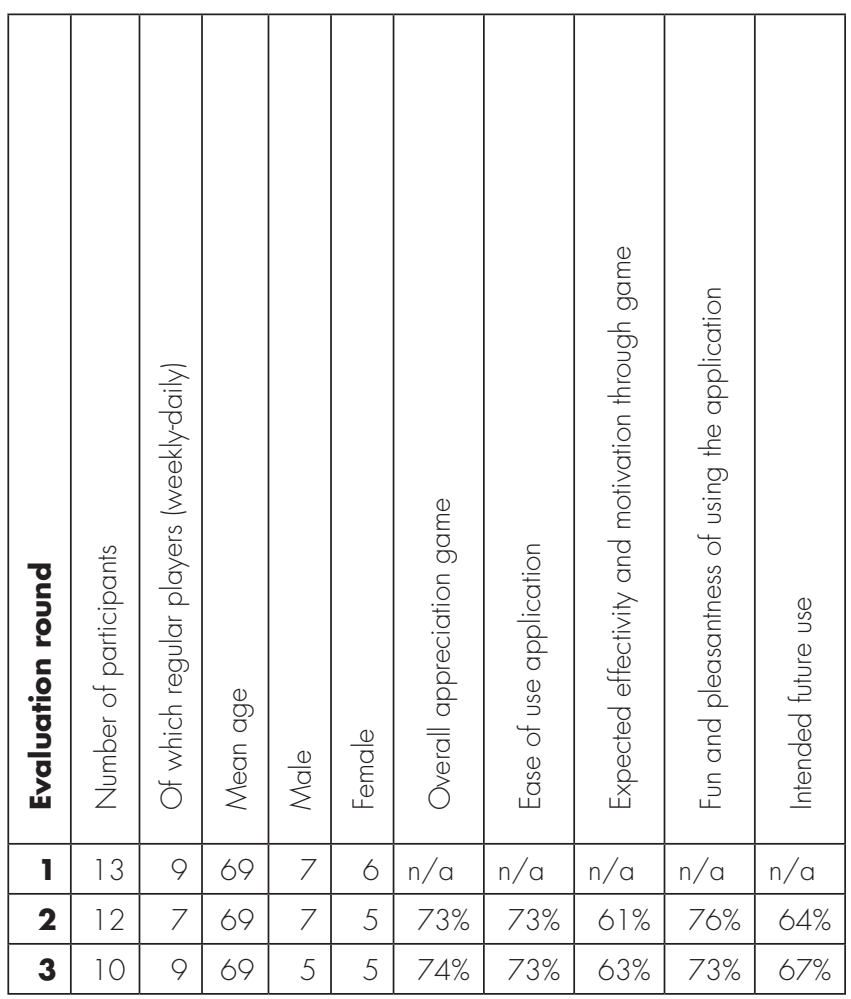

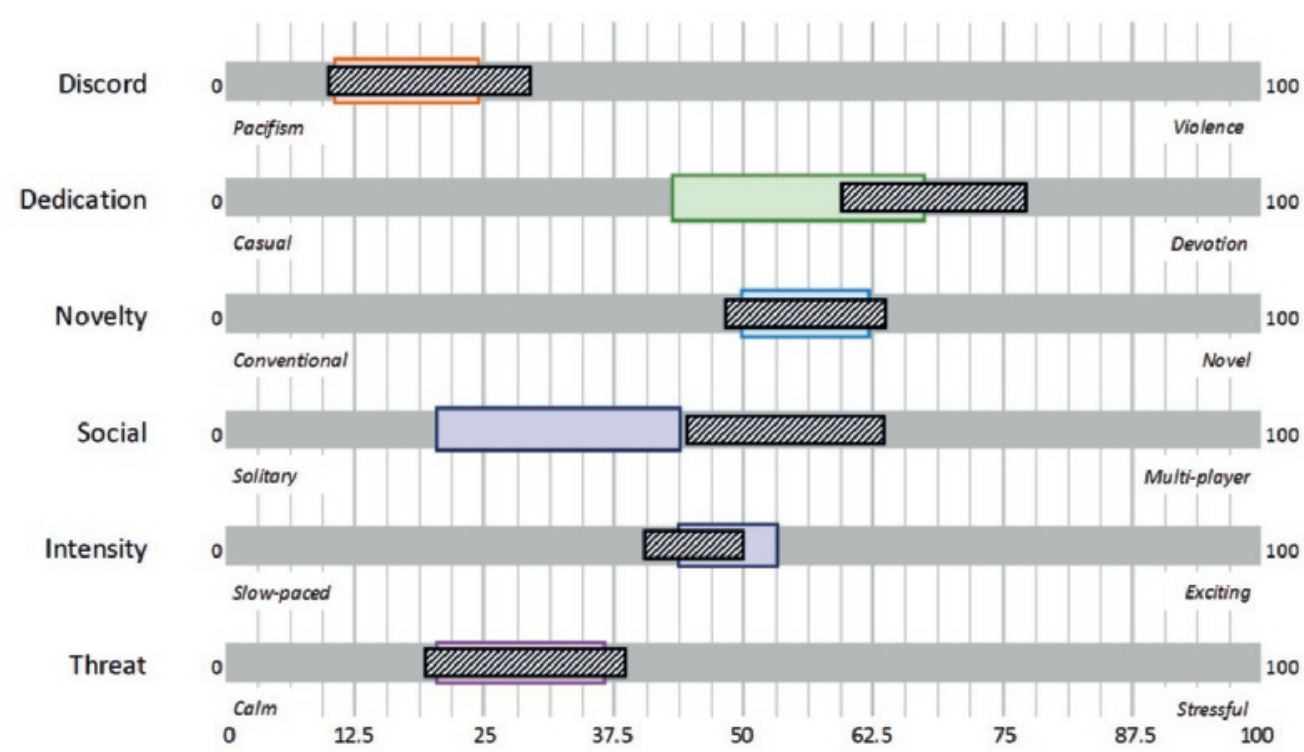

Figure 10 - User profile (perception: taller solid bars, appreciation: shorter hatched bars) 
In the third and final round the prototype game was used in the daily living environment for six weeks, again using simulated exercise. The scores that the questionnaire returned are very close to those of the prior session. Five people would recommend the gamified platform to peers, five people would recommend the underlying exercises to peers. One participant mentioned sometimes having difficulty starting the game and one found that playing was fatiguing for the eyes (no playing time mentioned). Six participants would like to continue using the game. Challenges and goals were appreciated by the majority of people, playing the mini-games was again considered the most motivating aspect of the game. The crop field was appreciated least. During the final evaluation round, no new usability issues emerged and no further errors occurred that hindered use of the online application.

As described, the perception and appreciation of participants on different aspects of game content were measured in each of the evaluation rounds 26 participants returned complete results). Answers were given using a 5-point VAS scale and transposed to percentages before analysis. The $95 \%$ intervals were calculated and visualised in a graph (fig. 10). This figure helps to gain insight in how the game content is perceived by the user, related to the appreciation of the user of this same content. Thus, we can quickly analyse which aspects of the game are likely to motivate the user to play, and which aspects should be revisited in a future version. In our pre-studies we noticed a clear difference between the measured perception (or interpretation) of aspects of a game that was tested and the measured appreciation for these aspects by the user. In testing the PERSSILAA game, the scores of perception and appreciation did not show such large discrepancies. The difference between the two average values for each of the domains las explained in section 3.11) was smaller than 4/100 for all domains except dedication and social, for which the differences were around 20/100. We therefore carefully assume that the guidelines we set in concluding the end-user research described in section 3.1 have helped us create attractive game content for the (computer literate) older adult user. As stated earlier, the social component deserves further exploration.

\section{5| Discussion}

This study provided insight in the development of game-based eHealth in practice from front to end. We hope that this article aids researchers and designers of future game-based applications. We introduced four phases in the process towards game-based eHealth; end-user research, conceptualisation, creative design and refinement. These phases can be integrated in general system development cycles (as e.g. $[49,50]$ ) or in specific frameworks for the development of eHealth applications [51]. Our case study demonstrated the application of the proposed 
game design process and resulted in a complete game-based eHealth application. Furthermore, user evaluations of the prototype application indicate that results from end-user research and consequential strategies for long-term engagement led to game content that is engaging to the older adult end user, supporting the value of our approach. We believe that by offering transparency on our approach of the design process and by providing practical examples we contributed to opening up the black box of game design that supports engagement in these applications.

\section{Game-based design process: limitations and future research}

Our iterative, four-phase method for game design gives prominence to the development of the actual game content within the overall eHealth application development. In a practical sense, however, such methods may also pose difficulties, as related work shows. For example, Hussain et al. state that priority conflicts arose from stakeholders less acquainted with agile approaches [52]. In our case study, we recognised this issue and responded to it by prioritising stakeholder influence in the design process in advance. This helped ensure that creative game design was given the necessary consideration and prevent being overshadowed by technical requirements or demands from the healthcare perspective. While satisfying each stakeholder, space was created for the creative process leading to designing fun and attractive game content.

From applying the game design process in practice, we learned that engagement is subject to many prerequisites. For example, not only the content of the application itself must be engaging, it must provide an added value over the use of the underlying application alone, usability should not form a threshold, the game must be well-designed and well-made, and so on. However, end-user characteristics are also influential. We find an 'ideal' end user for game-based eHealth to be open to the technology, able to work with this technology without extensive instruction and support, and preferably enjoys games and play. Furthermore, eHealth can play a supporting role as this user intends to work on the health objectives offered [53]. Participants included in our studies were such ideal users: able to use the devices well and open to games and technology for use in eHealth. Therefore, they stand model for the older adult of the future rather than being representative for most older adults at the time of writing (2018). The game-based PERSSILAA platform and design recommendations are therefore no guarantee for success when applied to use situations with different (healthcare) objectives or population samples. Repetition of the game-based design process as presented can (and should) lead to different requirements for game design when applied elsewhere. 


\section{Case study: limitations and future research}

In the case study, the game-based version of the eHealth application was evaluated in a simulated setting (phase 4 of the game design process: refinement). The full roll-out of the finalised product will occur after concluding the game-based design process, in the implementation phase (fig. 1). The performance of the game environment in terms of motivation and engagement has to be explored in its actual use situation in future studies. Pilot testing may lead to new insights affecting any of the prior development cycles. Follow-up research topics may include both evaluation of the developed game-based application, in terms of user experience and satisfaction in real-world setting (including real exercises). Also, exploration of future functionalities, in terms of social interaction, user-generated content and expansion of existing features for prolonged use times may contribute to sustaining engagement over time.

Results from phase 1 (end-user research investigating game preferences of end users and specifications from the use context of the envisioned application) indicated that the obvious gaming preferences of the older adult based on current gaming behaviour (e.g. Scrabble and cards) may not lead to successful concepts for gamebased eHealth. As eluded in the introduction of this paper, we therefore approached investigating potentially engaging content from the context of entertainment games and game design (De Vette et al., 2015a). While these are not restricted by underlying 'serious' goals and purely aim for a satisfying experience, future research should focus on gathering additional knowledge on how to determine preferences for game content in end users, to be used in current and future game-based or gamified eHealth applications (De Vette et al., submitted 2019).

Note: Multimedia appendices could not be included in this thesis. 

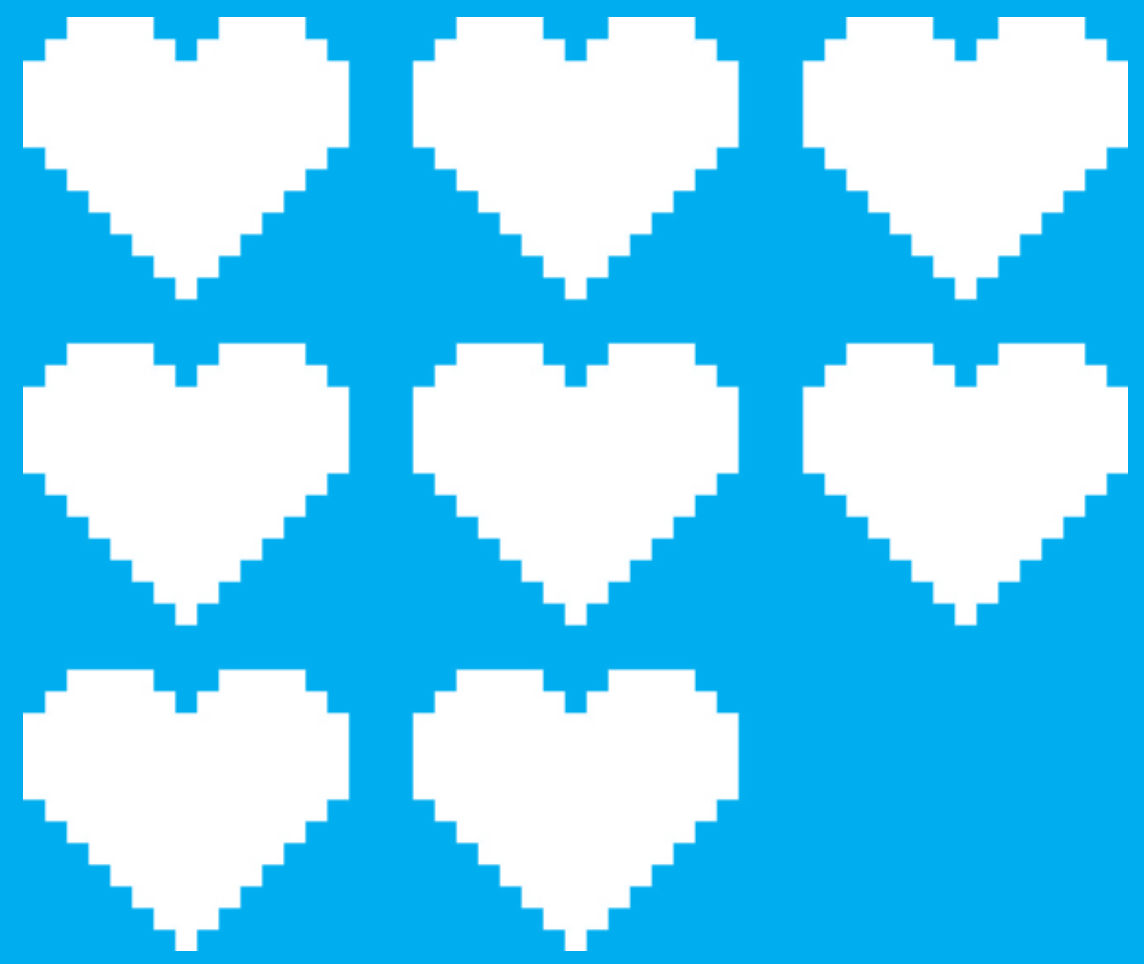


\section{Overview}

The aim of this thesis was to research how to design game-based applications that engage elderly users in eHealth. First, we framed a method to capture key game content characteristics that motivate people to play. This resulted in the newly developed "6D framework", which enables analysis of the motivating content of games as well as the preferences of users for this content (Chapters 2-5). As such, the framework offers a practical tool for designers of game-based applications in determining a motivational strategy by means of game elements. The framework can be applied to any target group, and was used in this research mainly, but not exclusively, to explore and concretise the gaming motivation of older adults. Secondly, this knowledge was applied in the design trajectory of game-based design within the development of eHealth applications (Chapters 6-7). We developed 'WordFit' to support users to maintain a healthy daily step goal, using an activity tracker and smartphone application. The development of 'Stranded!', a game-based self-management platform to counter frailty in older adults, resulted from demonstrating the entire design trajectory in practice from start to end. This study is a practical guidance on how to develop engaging game-based design that specifically addresses the game preferences of the older adult.

In this chapter we place the findings of this thesis in a broader perspective. We discuss these findings along the progressing stages of development of the gamebased application, from preliminary end-user research on suitable game content to concept design up to the actual game-based eHealth application that is ready for implementation in practice. We thereby shed light on the aspects that 1) are essential for correctly interpreting the gaming motivation of the user in order to create engaging game content, 2) create the conditions for successful game-based design as an engagement strategy and 3) need future investigation to make the implementation of developed game-based eHealth applications in practice a success as well.

\section{1| A recipe for engagement}

The literature review (Chapter 2) indicated a gap in knowledge on how to address the older adult through game-based concepts. It showed that evidence-based examples of gamified applications are scarce, and theoretical frameworks and user classifications to support the development of engaging gamification concepts offering practical methods are lacking. Furthermore, common methods to create gamified applications overly simplified game mechanisms, thereby disregarding underlying psychological foundations of motivation. The 6D framework, as introduced in this thesis, contributes to a solution for this challenge. The framework enables designers to create an overview of the motivating aspects of games and 
assess the user's preferences for these aspects in a desired level of detail, without being restricted to superficial game features (such as points and rewards). Applying the framework in practice therefore supports creating, comparing and evaluating strategies for the design of new game-based applications or adaptation of existing game concepts to address specific users.

The 6D framework was applied in practice to assess and map the content that motivates older adults to play (Chapter 4, 6-7). A surprising preference was found for what could be classified as 'high novelty content'. Instead of preferring the wellknown - many older adults played digital versions of conventional card and board games - they chose for content that triggered their curiosity, was fantastical and artistically designed when such games were made available to them. In summary, a solid amount of challenge, preferably intellectual, was appreciated more than a trivial gameplay style. The results also confirmed the expected importance of positivism, (extreme) non-violence and manageable playing tempo in realising engaging content. A question that remained unanswered was how to deal with content on the social dimension. Results from subsequent studies were contradicting, and while most participants indicated that an interest in social play lacked we cannot be conclusive on this aspect. We hypothesise that gaming is not yet interweaved with daily life as it is for younger generations, and that older adults strictly separate real-life social contact from the digital world. This is in line with findings of a study from Nap et al. [1] who concluded that older adults were simply not interested in playing digital games with others, but in contrast to research by De Schutter et al. [2] who suggest to emphasise connectedness in games through additional multiplayer options.

Through exploring these game preferences of older adults and creating new gamified applications accordingly, our findings showed that simply gathering information on the current game preferences and habits of older adults does not necessarily provide useful information for the designer, nor automatically results in a satisfying game concept for this end user (Chapters 3,6). To summarise, the immensely popular word-game Scrabble did not lead to a new game concept that was positively appreciated by the end user (Chapter 4). Possibly due to differences in specific lifetime experiences and world knowledge between generations, we found a divergence in the perception of game content of various target groups and the difficulties for the designer to adjust for these differences in advance (Chapters 3-5). Examples are the differences in perceived gameplay speed and level of violence between (the researcher or) designer, and that of our different target groups. Where children sometimes indicated playing habits of fast-paced and in our eyes often disturbingly violent video games, the older participants strongly disliked content we would barely recognise as being violent at all. Moreover, we found that a crucial user characteristic that colours the perception of the user on the presented game content is prior experience with video games (Chapter 3-4). Many older adults 
preferred to continue their old playing habits in digital formats; as also described by Brown [3]. They remained unaware of modern day video games, despite the fact that these may be enjoyable for them as well. This offset in experience between users of this target group, determining the affinity with game content presented in our studies, strongly influenced the measurements (Chapter 3-4). Therefore, in a field study targeting older adults we provided a selection of games to play beforehand of measuring their preferences (Chapter 4 ), or measured specific preferences of the content of the game-based applications developed after playing (Chapter 6-7). These findings show that the method for gaining knowledge on the end user is crucial when aiming for engaging game-based design. Not only in terms of using a suitable framework to explore game preferences but also in taking into account these specific user characteristics. In the interaction between the designer and the user, mediated by the 6D framework, there are two occurrences in which the context of the user is of influence on the framework outcomes: 1) before using the framework to assess user preferences, and 2) before interpreting and translating these measured preferences into (recommendations for) game design. Therefore, to correctly interpret the game preferences of the target group, both the appreciation of the user for game content and the perception of the user on this same content should be measured (fig. 1). Expanding the protocol for applying the 6D framework in practice with an additional survey measuring perception, as we have done in Chapter 6 and 7 , enables us to better predict if a game concept will lead to an engaging application and better evaluate its effectiveness in terms of engagement in implementation in daily practice. In addition, based on our results we propose to add a practical step to the process by offering more inexperienced end users modern day gaming experience. This may enable them to both expand and better express their preferences, and also provides game designers the opportunity to think beyond conventional game concepts.

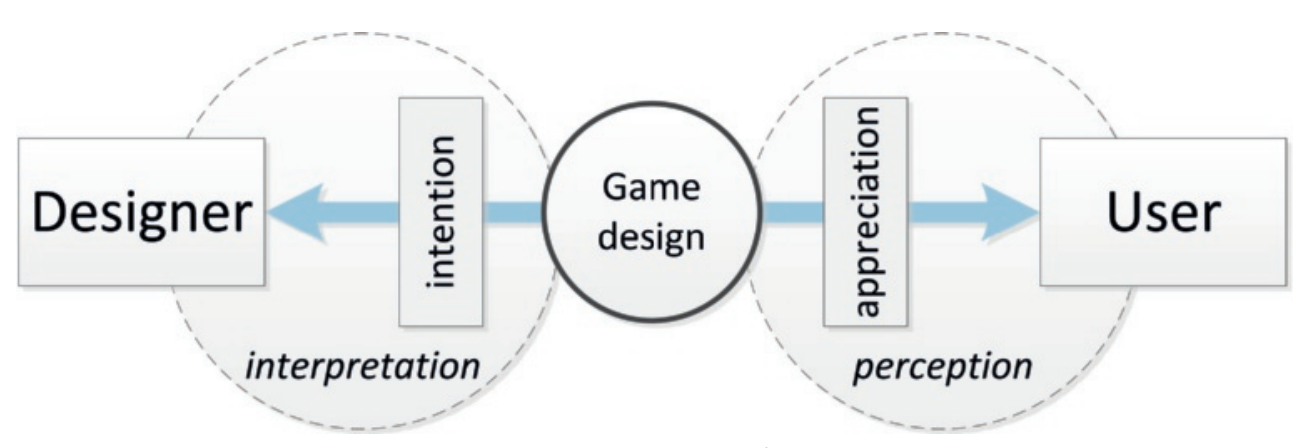

Fig. 1 - Interaction between designer and user mediated by the 6D framework 
Future research may target improving the framework through testing its reliability and external validity. In our studies (Chapter 5), we focused on the internal validity of the classification and practical demonstration of the framework. The classification may improve in terms of ease of measuring and interpreting preferences upon reducing potential overlap between the classification dimensions and refining the descriptive semantics used. From our findings we suspect that users often make use of just several criteria when they consider if the content of a game will satisfy them or not. In other words, some dimensions of the framework's classification may be more important than others. Moreover, a discrepancy can occur between the dimension that best predicts game preference and the dimension that is perceived as most influential to this game preference in the selection of actual games (see e.g. Chapter 5). Taking into account this possible unbalance on particular dimensions can give valuable leads on how to design content for the target user.

Continuation of research on the application of the framework can be made on games that involve a higher amount of physical interaction. In our research we have focused mainly on game-based design to support engagement in treatment. Underlying activities formed an unobtrusive mechanism for in-game progress for practical reasons as well. For example, step goals do not translate well into game controls (Chapter 6) and combining training performance on various subjects demanded a unified measure for progress (Chapter 7). However, as eHealth applications often present specific physical exercises, it may be worthwhile to scrutinise the framework's applicability to gaming formats that rely more directly on game-based feedback such as exergames (videogames intended for exercise). Examples are dancing games, racing simulators and gesture-controlled games (Wii fit, Kinect), as well as existing exergames.

The use of exploring and gaining insight in gaming motivation to create engaging content for diverse target groups is not restricted to application in non-entertainment context. We believe the 6D framework also holds the potential to inspire future developments in the consumer gaming industry. A vast part of the games on the market are created by designers that have the same demographics, designing from their own perspective and motivations. Looking at worldwide video game sales by genre of the past decade, 'shooter' and 'action' make up for roughly half of all games sold. While nowadays the old stereotype of gamer fades, the same does not hold true for the development of games. The choice in non-violent games that are interesting for adults, for example, is considerably smaller and often reserved to sports, simulation and casual games. The role of the 6D framework could be to investigate new markets and consumer groups, and better identify their preferences, reaching a wider and more diverse audience. In addition, as games are becoming part of mainstream media, developers may benefit from the framework's classification to complement traditional genre classifications. The 
framework can thereby be used as the basis for a recommender system. Currently, such a system is used by the company Quantic Foundry lbased on research by Yee et al.). By creating a large database and applying machine learning, predicting suitable games and content for end users can become easier and more effective. Both users and developers may benefit from such a system.

Besides emphasising the role of the user in the design process we do not want to leave the importance of the designer unaddressed. It cannot be stressed enough that the skills of designers are essential, and that in a multidisciplinary approach sufficient room should be given for a creative process to take place las we found in Chapter 7). The framework is merely the tool to create the blueprint for design. Just as a good musician is required to translate written chords into pleasant music, a good game designer is needed to create an attractive game concept from a profile of game preferences.

\section{2| Prerequisites for engagement}

After establishing the preferences for game content of the end user with the 6D framework, this knowledge can be translated into the game-based design concept. Where the previous section describes how to let game design itself be engaging to the user through creating the right content, this section elaborates on the factors from the intended use context that are conditional to the success of a game-based application in terms of engagement.

The results of our studies towards the preferences of older adults (Chapter 3, 4, 6, 7) showed the need of the older adult to justify spending time on games and gamebased applications. While participants in our studies were open to gaming, their opinion was that games are mainly good for pastime and, perhaps, socialisation. People worried that games would distract from underlying health aims, or simply waste their time. More easily accepted were games that cognitively challenged the player, as preferences we charted throughout this thesis reflect, because they have the clear purpose to help maintain a keen mind. In contrast to children, who almost automatically engage in play when there is an opportunity /see the setting of the field study in Chapter 4), our older participants weighed the benefits over the enjoyment of activities. Many participants declared that a certain game was definitely unsuitable for them, but perfect for that one friend or relative. On the other hand, in evaluating the game-based application in Chapter 7, feedback was positive on the expected effectivity and motivation through the game in combination with fun and pleasantness of using the application and intended future use. Moreover, in Chapter 6 we observed that while many participants did not see a link between the game-based application and its underlying healthcare goals, 
the game was drawing attention to the system itself in a pleasant way. It thereby perhaps offered a good excuse to indulge in playing a game, while stealthily increasing actual use of the application.

Still, this necessity to perceive benefits from gaming in older adults, as also described by IJsselsteijn et al. [4] and Melenhorst et al. [5], creates a substantial challenge in combining productive activities for underlying healthcare objectives with a medium that is not necessarily associated with productivity. In order to allow engagement of game-based concepts to be experienced, it must be clear to the user that the game does not derogate the underlying activity, and that gaming is deliberately applied to create a more enjoyable experience. We relate this prerequisite for engagement to technology acceptance. For technology acceptance in older adults, as described by Renaud et al. [6], there should be an intention to use followed by actual use. Both of these elements are influenced by various factors, of which most importantly usefulness, both perceived (beforehand of using the application) and confirmed (from using the application itself). Alternatively, when there is no perceived or confirmed usefulness, the technology is rejected. We illustrate this process in simplified form for engagement in game-based applications by including the factors applicable to our research (fig. 2). In case of game-based eHealth, technology acceptance would for example demand that the user has an open attitude towards the goals and technology of game-based eHealth, understands the value and benefits of the application, and is sufficiently computer literate to work with the devices and interfaces involved. Instead of mere acceptance of the technology, this would lead to allowing the game-based application to engage the user, reinforcing actual use.

According to our findings, design for older adults should particularly focus on reinforcing the usefulness of game-based design in eHealth applications. In our research (Chapter 6-7) we identified that the format of the game-based application influences the total experienced usefulness on three accounts: 1) the compatibility of the gameplay with the daily routine of the user, 2) social influence and 3) transparency of the in-game representation of underlying application objectives. Firstly, the application should combine an acceptable playing duration and playing time(s) during the day, and take into account the need for mobility of the user. In many fields, the importance of daily life integration is a universal concept in designing for older adults (for example Fisk et al. [7] in human factors, Roy et al. [8] in user centred design, technology supported active and healthy ageing, Ballegaard et al. [9], Carmichael et al. [10] and Vaziri et al. [1 1 ]). We found that the majority of people preferred playing at home and, as concluded from the study in Chapter 6, no participants felt the need to use the game-based application during the day or on the go. Instead, playing moments were moved to the evening, at home on the couch. Secondly, while field studies (Chapter 4 and 6) did not confirm the necessity 


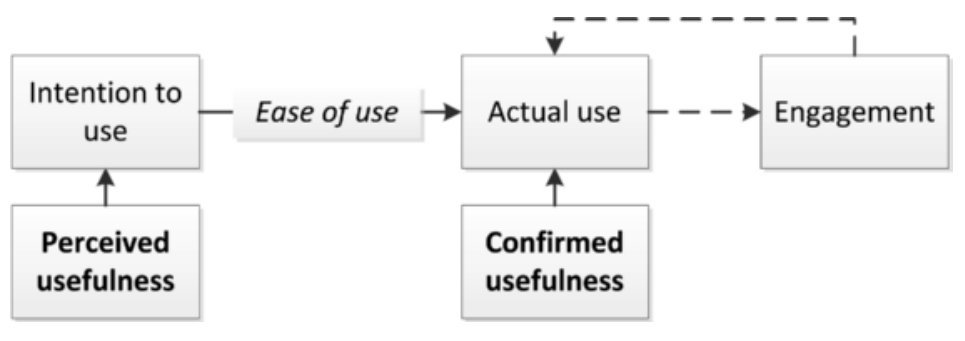

Figure 2 - Facilitating engagement in game-based applications (deduced from Renaud et al., 2008)

for social contact through game-based applications (the built-in cooperative modes in WordFit, for example, remained unused), we identified socialisation as a reason (or excuse) for older adults to play games. Also, participants actively recommended others their favourite tablet games. We expect that the perceived usefulness of applications can increase from setting peer examples. Thirdly, the representation of activities supporting the underlying health goals by gameplay should be easy to comprehend. If the in-game representation of activities supporting the health goal is not sufficiently intuitive, it may result into a loss of perceived usefulness. This includes direct game-based feedback (as in WordFit, Chapter 6) but also more unobtrusive mechanisms in which activities form an implicit part of gameplay las in Stranded!, Chapter 7).

Influencing the confirmed usefulness of the application, in terms of game design, are the ease of (learning and) use and robustness of the designed application. Specific factors influencing the ease of use of video games are mostly determined by agerelated change: cognition, perception and motor control [12-14]. Even while digital inclusion among older adults is particularly high in the Netherlands [15], and the group of seniors whom have used computers in their working life grows, 'digital anxiety' is still a common occurrence. In our experience from the field studies, older adults give an application a single chance to convince them. Whether that is a game or something else, this user does not take a trial and error approach and once a feeling of incompetence (the main cause of digital anxiety) is experienced their rejection of the technology is irreversible. Robustness should therefore be pursued, both in terms of the general function as in aesthetical value of the game and its supportive technology. While learning to use the application may not demand too much effort, care must be taken to not stigmatise from oversimplifying design and underestimating the skills of the user. It is a common misconception that target groups with lesser experience with modern day video games are less susceptible to well-made games in terms of graphics and overall aesthetical value las we showed in Chapter 4). We expect that the opposite is true, and recommend avoiding to approach the older adult with underdeveloped designs, for the same reason of irrevocable rejection of the design at hand and possibly even future developments. Trust of the end user in the developer of the application is of influence on the 
perceived usefulness as well [16]. This trust can be created by ensuring that the developer provides transparency on the need of the user that the application is meant to fulfil, and the purpose of gaming as a support mechanism. Taking care that the older adult feels recognised in this need, by confirming that his or her personal needs are heard and taken into account seriously, may greatly improve the (intention to) use the applications developed [17]. Moreover, trust can be increased by involving healthcare professionals [18], whom are usually highly valued by the end user such as their general practitioner. On the account of game design, introducing older adult game designers that are able to develop game concepts from their perspective and those of their peers may be a valuable addition in the development of new game-based solutions.

Contributing to stimulating trust and circumventing the stigma of the futility of games at the same time, an alternative to mapping game preferences may be to explore the motivation of the end user for other, past and current, favourite activities outside of games. This reduces the problem of a lack of prior video game experience of the end user, or the incapability of game designers to get their design intentions synchronised with the preferences of the end user. At the same time, introducing a sense of reminiscence may reduce the feeling in users that an activity is forced upon them that they are not interested in. Designers may become less restricted by their convention of how games should be designed and challenged to think out of the box when creating new concepts. Alternatively, we may take on the challenge of utilising gaming specifically to counter digital anxiety and support technology acceptance, a field that to our knowledge is still completely unexplored.

\section{3| Towards game-based eHealth in practice}

In this thesis we aimed to answer the question how to design game-based applications to engage older adults in eHealth. The studies included in this thesis contribute to this answer mainly by aiding developers, designers and researchers in tailoring their design to the end user. We created a method to give developed game-based applications the highest possible chance of success in terms of engagement and acceptance by the end user, and addressed many factors from this context of use that influences the design process. Since our efforts are concluded before large scale practical use takes place, research is needed on further evaluating these strategies for engagement through applying the game-based application in its final context of use (as also mentioned in Chapter 7). We therefore propose that such future research would benefit from being included in an ongoing implementation strategy for the developed game-based application in daily practice, to increase the effectiveness of evaluation studies and thereby the chance of success of the eventual application in practice. 
The importance of considering the final context of use is illustrated by two observations, made in the evaluation of the game-based eHealth application described in Chapter 6. From the evaluation of the WordFit application IChapter 6), we found that in this setting there was little necessity for a game-based strategy to support adherence of the user. On the one hand participants involved were either already quite active or highly motivated to become more active, for instance after illness. Apart from the issue whether these participants enjoyed the game-based version of the application or not, engagement support was not needed even after the expected duration of three weeks before adherence would drop. On the other hand the basic application, with its main feature being a step counter, motivated users to such an extent that the game-based application was not needed. Still, on the other side of the spectrum were participants that considered themselves ljustly or not) sufficiently active, or who explained they had followed orders most of their lives and did not feel like doing so anymore.

During this evaluation, firsthand observations were made concerning the effort and time that people involved invested in putting the application into service. Therapists organised communication to colleagues, gave attention to the use of the MAGGY system during treatment sessions and took on the role of helpdesk in case of questions or problems. Family members and other caregivers learned about the application and technology involved as well. In practice, even when autonomous use is aimed for, an investment would have to be made in time and effort in order to gain time in the long run from shortening the care trajectory. Healthcare professionals that were involved in this evaluation study were open to the use of this technology and its implications, but also expressed their concerns about resistance from colleagues. Therefore, in order to gain the most from game-based eHealth technology, multiple stakeholders that need to invest (in units of time, effort, funding and workforce) should be taken into account on the road towards successful implementation.

In order to scale up game-based eHealth from prototype to a solid product that can be used in daily practice, Jansen-Kosterink et al. [19] identify the factors that should be analysed to successfully implement future eHealth technology. They state that these factors are determined by the robustness of the technology supported intervention itself, including technology used and clinical purpose, combined with the service configuration, which is the way the application is introduced in daily practice. According to the authors, proper evaluation of these aspects is essential to convince the various stakeholders of the added value of these solutions (in terms of increased quality of care and/or reduction of costs) and to arrive at sustainable implementation in daily practice. Broens et al. [20] propose a layered implementation model in which the focus of developments is gradually expanded during the maturation of the application. At the basis of this model are five categories: technology, acceptance, financing and organisation, and policy and legislation. Our research, described in 
this thesis, could be placed at this model's two most inner layers, where the focus lies on technological advancements and an incentive towards the acceptance layer is made.

From the findings in our research we can narrow down all future research questions into one: does applying game-based design indeed benefit healthcare? To persuade the stakeholders from all steps towards implementation in practice, including care facilities, government institutions and health insurance organisations, this question needs answering from more than one point of view. We propose that future research towards successful implementation of game-based eHealth in practice should be approached in two ways. Future research should focus, top-down, on 1) thorough empirical research to the effectiveness of game-based eHealth solutions in terms of clinical outcomes and, bottom-up, on 2) providing an added value through the game-based eHealth solution within the healthcare sector, in traditional and IT supported care, focused on increasing benefits for all stakeholders involved.

Despite these large implications for future research, and the many conditions that are set for the eventual success of game-based solutions, we are generally optimistic about the applications of games in healthcare. After all, we know now that games, when they are designed properly, can successfully engage older adults. In the meantime the demand for engagement strategies will likely increase. Game-based design in eHealth, and even eHealth itself, is still rapidly advancing. In order to evolve to a mature medium some time and effort should still be invested. When forces are combined there is much to gain. In our observations there is a particular point of interest in closing the gap between commercial (or even serious) gaming industry and academia. Technology that can be used in new interventions is advancing as well, and even the target group itself is evolving, perhaps even more rapidly. Computer literacy, accessibility and availability are increasing, as will this target group's affinity with games. The older adult in ten years will therefore be considerably different from today as a potential user of game-based eHealth. Lastly, we reason that while perhaps only a small number of people of the total target group benefits from game-based eHealth, their improvement is certainly well worth the effort. 

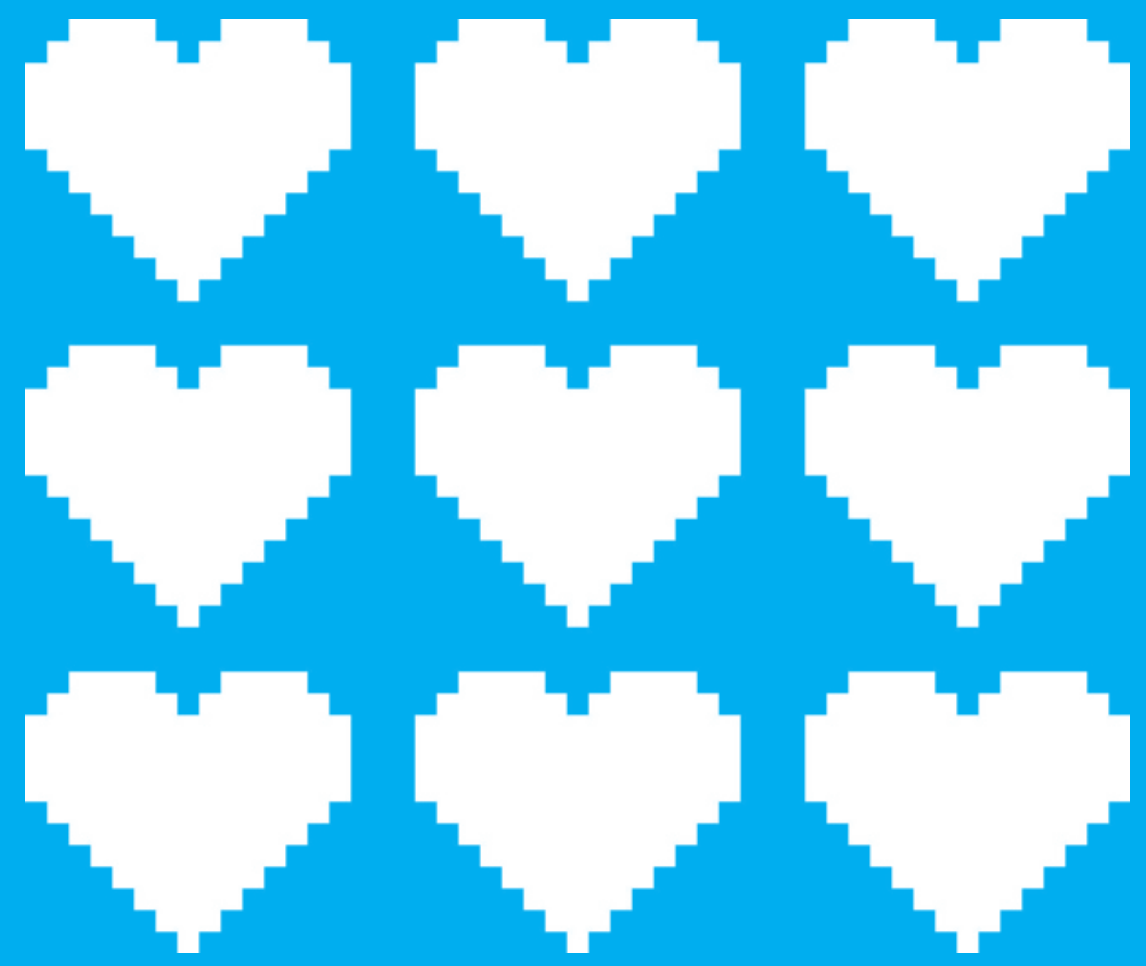
References
Summary
Samenvatting (summary in Dutch)
Dankwoord (acknowledgements in Dutch)

References
Summary
Samenvatting (summary in Dutch)
Dankwoord (acknowledgements in Dutch)

References
Summary
Samenvatting (summay in
Dankwoord (acknowled

$\&$

$$
\text { (h) }
$$

\&)

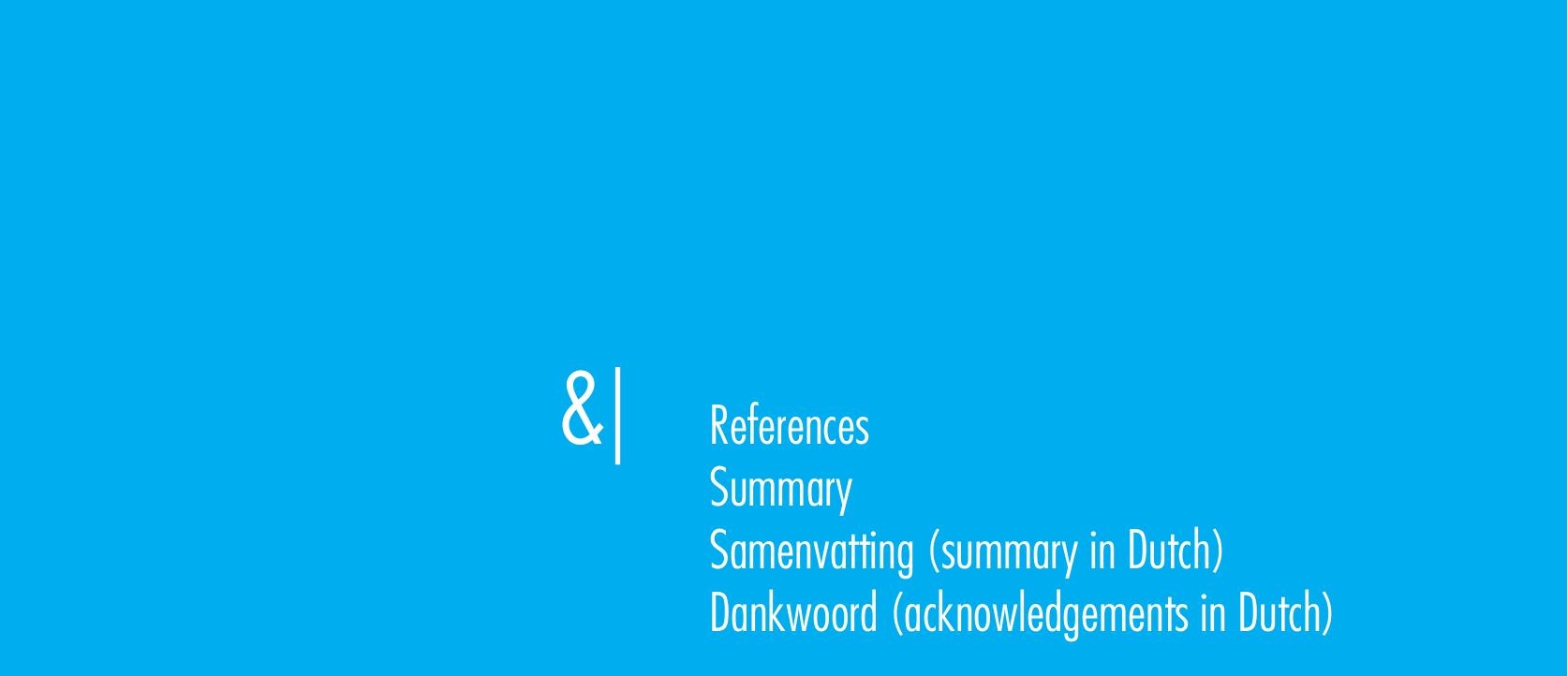

(1)

(1) 


\section{References}

\section{Chapter 1] General introduction}

1. Huizinga J. Homo Ludens. Proeve eener bepaling van het spel-element der cultuur. 1950

2. Abt CC. Serious Games. Viking Press, New York. 1970

3. Malone TW. Toward a theory of intrinsically motivating instruction. Cognitive Science, Volume 5, Issue 4, 1981, Pages 333-369, ISSN 0364-0213, https://doi.org/10.1016/S0364-0213181/80017-1

4. Deterding S, Dixon D, Khaled R, Nacke L. From Game Design Elements to Gamefulness Defining Gamification. In Proceedings of the 15th International Academic MindTrek Conference: Envisioning Future Media Environments (MindTrek '11). ACM, New York, NY, USA, 9-15. DOI: https://doi.org/10.1145/2181037.2181040

5. Hunicke R, LeBlanc M, Zubek R. MDA: A Formal Approach to Game Design and Game Research. In Proceedings of the Challenges in Games Al Workshop, Nineteenth National Conference of Artificial Intelligence, 2004

6. Werbach K, Hunter D. For the Win - Wharton Digital Press, 2012

7. Przybylski AK, Rigby CS, Ryan RM. A Motivational Model of Video Game Engagement. Rev Gen Psychol. 2010; 14(2): 154-66

8. Lepper MR, Greene D, Nisbett RE. Undermining children's intrinsic interest with extrinsic reward: A test of the "overjustification" hypothesis. J Pers Soc Psychol. 1973;28(1): 129-37

9. United Nations. World Population Prospects: the 2017 Revision. Retrieved from https://www.un.org/en/development/desa/population/publications

10. World Health Organization Europe (WHO). Healthy ageing. 2014

11. Eurostat. Population structure and ageing. ISSN 2443-82192018. 2018

12. Population Reference Bureau. World Population Aging: Clocks Illustrate Growth in Population Under Age 5 and Over Age 65, 2011

13. CBS. Gezondheid en zorggebruik; persoonskenmerken. Retrieved from statline.cbs. nl. 2018

14. RIVM. Chronische ziekten en multimorbiditeit. Retrieved from www. volksgezondheidenzorg.info. 2018

15. Eysenbach G. What is e-health? J Med Internet Res 200 1;3(2):e20. DOI: 10.2196/ jmir.3.2.e20

16. Bashshur RL, Shannon GW, Smith BR, Alverson DC, Antoniotti N, Barsan WG, Bashshur N, Brown EM, Coye MJ, Doarn CR, Ferguson S, Grigsby J, Krupinski EA, Kvedar JC, Linkous J, Merrell RC, Nesbitt T, Poropatich R, Rheuban KS, Sanders JH, Watson AR, Weinstein RS, ... Yellowlees P. The empirical foundations of telemedicine interventions for chronic disease management. Telemedicine journal and e-health: the official journal of the American Telemedicine Association, 20(9), 769-800. 2014

17. Groves P, Kayyali B, Knott D, Kuiken SV. The 'big data'revolution in healthcare: Accelerating value and innovation. 2016 
18. Hamine S, Gerth-Guyette E, Faulx D, Green BB, Ginsburg AS. Impact of mHealth chronic disease management on treatment adherence and patient outcomes: a systematic review. Journal of medical Internet research. 2015 Feb; 17/2)

19. Free C, Phillips G, Galli L, Watson L, Felix L, Edwards P, Patel V, Haines A. The effectiveness of mobile-health technology-based health behaviour change or disease management interventions for health care consumers: a systematic review. PLoS medicine. 2013 Jan 15;10(1):e1001362

20. Alpay L, van der Boog P, Dumaij A. An empowerment-based approach to developing innovative e-health tools for self-management. Health informatics journal. 2011 Dec; 17(4):247-55

21. Carey M, Noble N, Mansfield E, Waller A, Henskens F, Sanson-Fisher R. The role of eHealth in optimizing preventive care in the primary care setting. Journal of medical Internet research. 2015 May; 17(5)

22. Linton SJ, Andersson T. Can chronic disability be prevented? : A randomized trial of a cognitive-behavior intervention and two forms of information for patients with spinal pain. Spine. 2000 Nov 1;25(21):2825-31

23. Eysenbach G. The law of attrition. J Med Internet Res. 2005;7(1):e11. doi: 10.2196/imir.7.1.e11

24. Wangberg SC, Bergmo TS, Johnsen JA. Adherence in Internet-based interventions. Patient Prefer Adherence. 2008;2:57-65.

25. Neil AL, Batterham P, Christensen H, Bennett K, Griffiths KM. Predictors of adherence by adolescents to a cognitive behavior therapy website in school and communitybased settings. J Med Internet Res. 2009; 11 (1):e6

26. Christensen H, Griffiths KM, Farrer L. Adherence in internet interventions for anxiety and depression. J Med Internet Res. 2009; 1 1(2):e1 3. doi: 10.2196/jmir. 1194

27. Cugelman B, Thelwall M, Dawes P. Online interventions for social marketing health behavior change campaigns: a meta-analysis of psychological architectures and adherence factors. J Med Internet Res. 2011 ; 13(1):e17. doi: 10.2196/jmir. 1367

28. Kelders SM, Kok RN, Ossebaard HC, Van Gemert-Pijnen JE. Persuasive system design does matter: a systematic review of adherence to web-based interventions. Journal of medical Internet research, 14(6), e152. doi:10.2196/imir.2104. 2012

29. Illario M, Vollenbroek-Hutten M, Molloy DW, Menditto E, laccarino G, Eklund P. Active and Healthy Ageing and Independent Living. Journal of aging research, 2015,542183

\section{Chapter 2| Engaging elderly people in telemedicine through gamification}

1. WHO. Healthy ageing [Internet]. World Health Organization; [cited 2015 Apr 20]. [URL: http://www.euro.who.int/en/health-topics/Life-stages/healthy-ageing/ healthy-ageing Archived at: http://www.webcitation.org/6XvRTikMB] 
2. Przywara B. Projecting future health care expenditure at European level: drivers, methodology and main results [Internet]. Economic Papers 417. 2010. [URL: http:// ec.europa.eu/economy_finance/publications/economic_paper/2010/pdf/ ecp417_en.pdf Archived at: http://www.webcitation.org/6XvT7h8lg]

3. Jansen-Kosterink S. The added value of telemedicine services for physical rehabilitation. University of Twente. 2014. [ISBN:9789082319606] [PMID: 17725187]

4. Tabak M, Brusse-Keizer M, Valk P Van Der, Hermens H, Vollenbroek-Hutten M. A telehealth program for self-management of COPD exacerbations and promotion of an active lifestyle: a pilot randomized controlled trial. International journal of COPD. DovePress; 2014. p. 935-44. [DOI:10.2147/COPD.S60179]

5. Evering RMH. Ambulatory Feedback at Daily Physical Activity Patterns: A Treatment for the Chronic Fatigue Syndrome in the Home Environment? University of Twente; 2013. [DOl: 10.3990/1.9789036535120]

6. Huis in 't Veld RMHA, Kosterink SM, Barbe T, Lindegård A, Marecek T, VollenbroekHutten MMR. Relation between patient satisfaction, compliance and the clinical benefit of a teletreatment application for chronic pain. J Telemed Telecare. 2010; 16(6):322-8. [DOI:10.1258/itt.2010.006006] [ISBN:1758-1109 (Electronic)r 1 357-633X (Linking)] [PMID:20798426]

7. Gerling K, Masuch M. Exploring the potential of gamification among frail elderly persons. CHI 2011 Work Gamification Using Game Des. 2011;1-4. [ISBN:978 1450302685$]$

8. Bakkes S, Tan CT, Pisan Y. Personalised Gaming: A Motivation and Overview of Literature. Proc 8th Australas Conf Interact Entertain Play Syst '12. 2012;4. [DOI: 10.1 145/2336727.2336731] [ISBN:9781450314107]

9. Cordova DI, Lepper MR. Intrinsic motivation and the process of learning: Beneficial effects of contextualization, personalization, and choice. J Educ Psychol. 1996;88(4):715-30. [DOI: 10.1037/0022-0663.88.4.715] [ISBN: 19392176 (Electronic); 0022-0663 (Print)] [PMID:201]

10. Deterding S, Dixon D, Khaled R, Nacke L. From game design elements to gamefulness. Proc 15th Int Acad MindTrek Conf Envisioning Futur Media Environ - MindTrek ' 11. $2011 ; 9-11$. [DOI:10.1145/2181037.2181040] [ISBN:9781450308168]

11. Werbach K. Gamification - University of Pennsylvania | Coursera [Internet]. [cited 2015 Apr 20]. [URL: https://www.coursera.org/course/gamification Does not allow for WebCite archiving]

12. Zichermann G, Cunningham C. Gamification By Design. O'Reilly Media, Sebastopol, CA.; 2011 . [ISBN:978 1449397678] [PMID:21949098]

13. Malone TW. Heuristics for designing enjoyable user interfaces: Lessons from computer games. Proc 1982 Conf Hum factors Comput Syst. 1982;63-8. [DOI: 10.1 1 45/800049.801756] [ISBN:089391 1461]

14. Werbach K, Hunter D. For the Win: How Game Thinking Can Revolutionize Your Business. Wharton Digital Press, Philadelphia, PA.; 2012. 149 p. [ISBN:9781613630228] 
15. Huotari K. Defining Gamification - A Service Marketing Perspective. Proc 15th Int Acad MindTrek Conf Envisioning Futur Media Environ. 2012;17-22. [DOI: 10. $1145 / 2393132.2393137]$ [ISBN:9781450316378]

16. Chou Y. A comprehensive list of $90+$ gamification cases with $\mathrm{ROI}$ stats [Internet]. 2015 [cited 2015 Jul 22]. [URL: http://www.yukaichou.com/gamificationexamples/gamification-stats-figures/\#.Va9lGPntBd Archived at: http://www. webcitation.org/6aDet6OK9]

17. Gartner inc. Gartner Reveals Top Predictions for IT Organizations and Users for 2013 and Beyond [Internet]. 2012 [cited 2015 Jul 22]. [URL: http://www.gartner.com/ newsroom/id/2211115 Archived at: http://www.webcitation.org/baDfackwr]

18. Meloni W, Gruener W. Gamification in 2012. M2 Res [Internet]. 2012;25. [URL: http://gamingbusinessreview.com/wp-content/uploads/2012/05/Gamificationin-20 1 2-M2R3.pdf]

19. Ryan, M., Sleigh, A., Soh, K.W., Li Z. Why gamification is serious business [Internet]. 2015 [cited 2015 Jul 22]. [URL: https://www.accenture.com/za-en/ insight-outlook-why-gamification-is-serious-business.aspx Archived at: http://www. webcitation.org/baDg6JUQB]

20. Cetin L. The SAP Community Network: How to Use Gamification to Increase Engagement. 2013; [URL: http://www.enterprise-gamification.com/index. php?option=com_content\&view=article\&id $=160$ :the-sap-community-network-how-touse-gamification-to-increase-engagement\&catid=15\&ltemid=22\&lang=en Archived at: http://www.webcitation.org/6aDgGbApu]

21. Zichermann G. Gamification: The Hard Truths. 2013; [URL: http://www. huffingtonpost.com/gabe-zichermann/gamification_b_2516376.html Archived at: http://www.webcitation.org/6aDgMo6Pa]

22. Takahashi D. Badgeville's Kris Duggan: Six frameworks can gamify employee and customer engagement (interview) [Internet]. 2012 [cited 2015 Apr 20]. [URL: http:// venturebeat.com/2012/05/30/badgeville-kris-duggan-interview/ Archived at: http://www.webcitation.org/6XvUlxilW]

23. Badgeville: The \# 1 Gamification Platform for the Enterprise [Internet]. [cited 2015 Apr 20]. [URL: https://badgeville.com/ Archived at: http://www.webcitation. org/6XvUyaDmQ]

24. Gartner inc. Gartner Says by 2014, 80 Percent of Current Gamified Applications Will Fail to Meet Business Objectives Primarily Due to Poor Design. 2012; [URL: http://www.gartner.com/newsroom/id/2251015 Archived at: http://www. webcitation.org/baDgYij3Z]

25. Robertson M. Can't play, won't play - Blog: Hide\&Seek [Internet]. 2010 [cited 2015 Apr 20]. [URL: http://hideandseek.net/2010/10/06/cant-play-wont-play Archived at: http://www.webcitation.org/6XvYdsA2p]

26. Bogost I. Persuasive Games: Exploitationware - Gamasutra [Internet]. 2011 [cited 2015 Apr 20]. p. 4. [URL: http://www.gamasutra.com/view/feature/134735/ persuasive_games_exploitationware.php Archived at: http://www.webcitation. org/6XvYoDaCl] 
27. Bogost I. Gamification is Bullshit [Internet]. 2011 [cited 2015 Apr 20]. [URL: http:// bogost.com/writing/blog/gamification_is_bullshit/ Archived at: http://www. webcitation.org/6XVZOUBql]

28. Skinner BF. The behavior of organisms: an experimental analysis [Internet]. AppletonCentury; 1938. [URL: hitp://psycnet.apa.org/psycinfo/1939-00056-000]

29. Lepper MR, Greene D, Nisbett RE. Undermining children's intrinsic interest with extrinsic reward: A test of the "overiustification" hypothesis. J Pers Soc Psychol. 1973;28(1):129-37. [DOl:10.1037/h0035519] [ISBN:00223514r1939-1315]

30. Carlson NR, Miller H, Heth CD, Donahoe JW, Martin GN. Psychology: The Science of Behavior. 7th Editio. Prentice Hall PTR; 2010. 644 p. [ISBN:020508706X]

31. Brickman P, Campbell D. Hedonic relativism and planning the good society. Adapt theory a Symp. 1971;287-302.

32. Aparicio AF, Vela FLG, Sánchez JLG, Montes JLl. Analysis and application of gamification. Proc 13th Int Conf Interacción Pers - INTERACCION '12. 2012; 1-2. [DOI: 10.11 145/2379636.2379653] [ISBN:9781450313148]

33. Ryan RM, Deci EL. Intrinsic Motivation and Self-Determination in Human Behavior. New York: Plenum; 1985. [ISBN:0-306-42022-8]

34. Nicholson S. A User-Centered Theoretical Framework for Meaningful Gamification A Brief Introduction to Gamification Organismic Integration Theory Situational Relevance and Situated Motivational Affordance. GameslearningSociety 80. 2012;

35. Norman DA. The design of everyday things. Second edi. Basic Books, New York; 1988. [ISBN:0465067107]

36. Deterding S. Situated motivational affordances of game elements: A conceptual model. Chi 2011. 2011;3-6. [DOI:ACM 978-1-4503-0268-5/11/05] [ISBN:9781450302685]

37. Wilson P. Situational relevance. Inf Storage Retr 9. :457-71.

38. Rose DH, Meyer A, Strangman N, Rappolt G. Teaching every student in the digital age: universal design for learning. Association for Supervision and Curriculum Delevopment, Alexandria; 2002. [ISBN:0-871 20-599-8]

39. Prochaska JO, Marcus BH. The transtheoretical model: Applications to exercise. Adv Exerc adherence. 1994;161-80.

40. Sakamoto M, Nakajima T, Alexandrova T. Value-based design for gamifying daily activities. Lect Notes Comput Sci. 20 1 2;7522 LNCS:42 1-4. [DOI: $10.1007 / 978-$ 3-642-33542-6_43] [ISBN:9783642335419]

41. Tabak M, Dekker-van Weering MGH, Van Dijk H, Vollenbroek-Hutten MMR. Promoting Daily Physical Activity by Means of Mobile Gaming: A Review of the State of the Art. Games Health J. 2015;4(6). [DOl:10.1089/g4h.2015.0010] [PMID:26397179]

42. Link MW, Lai J, Bristol K, C†A. Not So Fun? The Challenges of Applying Gamification to Smartphone Measurement. DUXU 2014 part IV. 2014;319-27.

43. Minge M, Bürglen J, Cymek DH. Exploring the Potential of Gameful Interaction Design of ICT for the Elderly. Commun Comput Inf Sci. 2014;435 PART I(2004):304-9. [DOI: $10.1007 / 978-3-31$ 9-07854-0_54] [ISBN:978-3-319-07853-3] 
44. IJsselsteijn W, Nap HH, de Kort Y, Poels K. Digital Game Design for Elderly Users. Proc 2007 Conf Futur Play. 2007; 17-22. [DOI: 10. 1 145/1328202. 1328206] [ISBN:9781595939432]

45. Bartle R. Player Type Theory: Uses and Abuses (lecture) [Internet]. 2012 [cited 2015 Apr 20]. [URL: http://youtu.be/ZlzLbE-93nc Archived at: http:/ / www.webcitation. org/6XvZUrAVZ]

46. Bartle RA. Hearts, clubs, diamonds, spades: Players who suit MUDs [Internet]. 1996 [cited 2015 Apr 20]. [URL: http://mud.co.uk/richard/hcds.htm Archived at: http://www.webcitation.org/6XvZlirzF]

47. Marczewski A. A New Perspective on the Bartle Player Types for Gamification [Internet]. 2012 [cited 2015 Apr 20]. [URL: http://www.gamification.co/2013/08/12/anew-perspective-on-the-bartle-player-types-for-gamification/ Archived at: http:// www.webcitation.org/6XvZuwUtK]

48. Vandenberghe J. The 5 Domains of Play: Applying Psychology's Big 5 Motivation Domains to Games [Internet]. 2012 [cited 2015 Apr 20]. [URL: http://www. gdcvault.com/play/1015364/The-5-Domains-of-Play Archived at: http:/ /www. webcitation.org/6Xva2AWDK]

49. Yee N. Motivations of play in MMORPGs. Proc DiGRA 2005 Conf Chang Views Worlds Play. 2005;

50. Przybylski AK, Rigby CS, Ryan RM. A motivational model of video game engagement. Rev Gen Psychol. 20 10; 14(2): 154-66. [DOI: 10. 1037/a00 19440] [ISBN: 10892680 1939-1552]

51. Hunicke R, LeBlanc M, Zubek R. MDA: A Formal Approach to Game Design and Game Research. Work Challenges Game Al. 2004; 1-4. [DOI: 10.1.1.79.4561]

52. Lazzaro N. Why We Play Games: Four Keys to More Emotion Without Story. Game Dev Conf. 2004; 1-8. [DOI: 10.1111 1/i.1464-410X.2004.04896.x]

53. Dixon D. Player Types and Gamification. CHI 2011 Work Gamification Using Game Des Elem NonGame Context. 201 1;12-5. [DOI:ACM 978-4503-02685/11/05] [ISBN:9781450302685]

54. Marczewski A. A User Type Framework for Gamification Design. 2013 [cited 2015 Apr 20]; [URL: http://www.gamified.uk/user-types/ Archived at: http://www. webcitation.org/6XvcmkQ9e]

55. Kyatric. Bartle's Taxonomy of Player Types (And Why It Doesn't Apply to Everything) - Tuts+ Game Development Article [Internet]. 2013 [cited 2015 Apr 20]. [URL: http:/ / gamedevelopment.tutsplus.com/articles/bartles-taxonomy-of-player-typesand-why-it-doesnt-apply-to-everything-gamedev-4173 Archived at: http://www. webcitation.org/6Xvcy9GYp]

56. Tredoux G. Francis Galton as Anthropologist [Internet]. [cited 2015 Apr 20]. [URL: http://galton.org/anthropologist.htm Archived at: http://www.webcitation. org/6XvdOlx8n]

57. Allport GW. Personality: a psychological interpretation. Am J Sociol. 1937;45(1):120-3. 
58. McCrae RR, Costa PT. Validation of the five-factor model of personality across instruments and observers. J Pers Soc Psychol. 1987;52(1):81-90. [DOl:10.1037/00223514.52.1.81] [ISBN: 1939-1315] [PMID:3820081]

59. Costa Jr. PT, McCrae RR. Four ways five factors are basic. Pers individ Diff. $1991 ; 13(6): 653-65$.

60. Bouchard TJ, Loehlin JC. Genes, Evolution, and Personality. Behav Genet. $2001 ; 31(3): 243-73$.

61. Gurven M, von Rueden C, Massenkoff M, Kaplan H, Lero Vie M. How universal is the Big Five? Testing the five-factor model of personality variation among foragerfarmers in the Bolivian Amazon. J Pers Soc Psychol. 2013;104(2):354-70. [DOI: 10.1037/a0030841] [ISBN: $1939-1315$ (Electronic)rO022-35 14 (Linking)] [PMID:23245291]

62. McCrae RR. Cross-cultural research on the five-factor model of personality. Online readings Psychol Cult. 2002;4(4):1-12. [DOI:10.9707/2307-0919.1038] [ISBN:9780984562701]

63. McCrae RR, Terracciano A. Universal features of personality traits from the observer's perspective: data from 50 cultures. J Pers Soc Psychol. 2005;88(3):547-61. [DOI: 10.1037/0022-35 14.88.3.547] [ISBN:0022-3514] [PMID: 15740445]

64. Schmitt DP, Allik J, McCrae RR, Benet-Martinez V. The Geographic Distribution of Big Five Personality Traits: Patterns and Profiles of Human SelfDescription Across 56 Nations. J Cross Cult Psychol. 2007;38(2):173-212. [DOI: 10.1 $177 / 0022022106297299]$ [ISBN:0022-0221] [PMID:4065]

65. Orii R, Vassileva J, Mandryk RL. Modeling the efficacy of persuasive strategies for different gamer types in serious games for health. User Model User-adapt Interact. 2014;1-35. [DOl: 10.1007/s 11 257-014-9149-8]

66. Borders J. Relationship Between Personality and Video Game Preferences. 2012;

67. Zammitto V. Game research, measuring gaming preferences. Appl Artif Intell. 2009; (May): 15-6. [DOI: 10.1 145/1639601.1639611] [ISBN:9781605586854]

68. Zammitto VL. Gamers' Personality and their Gaming Preferences. Sch Interact Arts Technol. 2010;M.Sc Thesi: 147.

69. Teng C. Online Game Player Personality and Real-life Need Fulfillment. Int J Cyber Soc Educ. 2009;2(2):39-50.

70. Despain W. 100 Principles of Game Design. New Riders, Pearson Education; 2013. [ISBN:0-321-90249-1]

71. Yee N. Motivations for play in online games. Cyberpsychol Behav. 2006;9(6):7725. [DOI: 10. 1089/cpb.2006.9.772] [ISBN: 1094-9313] [PMID: 17201605]

72. Fua KC, Gupta S, Pautler D, Farber L. Designing Serious Games for Elders. Proc Int Conf Found Digit Games. 2013;291-7.

73. Brox E, Hernandez JEG. Exergames for elderly: Social exergames to persuade seniors to increase physical activity. 2011 5th Int Conf Pervasive Comput Technol Healthc Work. 201 1;546-9. [DOI: 10.4108/icst.pervasivehealth.201 1.246049] [ISBN:978-1-936968-14-5] 
74. Billis AS, Konstantinidis El, Mouzakidis C, Tsolaki MN, Pappas C, Bamidis PD. A game-like interface for training seniors' dynamic balance and coordination. IFMBE Proc. 2010;29:691-4. [DOI:10.1007/978-3-642-13039-7_174] [ISBN:9783642130380]

75. Gerling KM, Schild J, Masuch M. Exergame design for elderly users: the case study of SilverBalance. Proc 7th Int Conf Adv Comput Entertain Technol - ACE '10. 2010;66-9. [DOI:10.1145/1971630.1971650] [ISBN:9781605588636] [PMID: 15123609$]$

76. Brauner P, Calero Valdez A, Schroeder U, Ziefle M. Increase Physical Fitness and Create Health Awareness through Exergames and Gamification. The Role of Individual Factors, Motivation and Acceptance. Proc SouthCHI 2013 , LNCS 7946. $2013 ; 349-62 . \quad$ [DOI:10.1007/978-3-642-39062-3_22] [ISBN:978-3-64239061-6]

77. Fogg B. A behavior model for persuasive design. Proc 4th Int Conf Persuas Technol - Persuas '09. 2009;1. [DOI:10.1145/1541948.1541999] [ISBN:9781605583761]

78. Oinas-kukkonen H, Harjumaa M. Persuasive Systems Design: Key Issues, Process Model, and System Features. Commun Assoc Inf Syst. 2009;24(28):485-500. [ISBN: 15293181 ] [PMID:45267822]

79. Kelders SM, Kok RN, Ossebaard HC, Van Gemert-Pijnen JEWC. Persuasive system design does matter: A systematic review of adherence to web-based interventions. J Med Internet Res. 2012;14(6):1-24. [DOl: 10.2196/imir.2104] [PMID:23151820]

80. Cugelman B. Gamification: What It Is and Why It Matters to Digital Health Behavior Change Developers. JMIR Serious Games. 2013 Dec 12;1(1):e3. [DOI: 10.2196/ games. 31 39] [PMID:25658754]

81. ConsolvoS, McDonaldDW, LandayJ a. Theory-driven design strategies for technologies that support behavior change in everyday life. Proc 27th Int Conf Hum factors Comput Syst - CHI 09. 2009;405-14. [DOI:10.1145/1518701.1518766] [ISBN:9781605582467]

82. Csikszentmihalyi M, Harper, Row. FLOW: The Psychology of Optimal Experience. Acad Manag Rev. 1990; [DOI: 10.5465/AMR. 1991 .4279513] [ISBN:0060920432] [PMID: 112$]$

83. Schell J. The Art of Game Design, a book of lenses. 2008. Chapter: The Flow Channel p. [ISBN:0123694965]

\section{Chapter 31 Exploring personality and game preferences in the younger and older population: a pilot study}

1. Jansen-Kosterink S. The added value of telemedicine services for physical rehabilitation. University of Twente. 2014. [ISBN:97890823 19606] [PMID: 17725187] 
2. Pavel M, Jimison $H$, Hayes $T$, Cooper R a, Marottoli R. Technologies for an Aging Population. Bridg Natl Acad Eng. 2009;39(1):68.

3. Huis in 't Veld RMHA, Kosterink SM, Barbe T, Lindegård A, Marecek T, VollenbroekHutten MMR. Relation between patient satisfaction, compliance and the clinical benefit of a teletreatment application for chronic pain. J Telemed Telecare. 2010; 16(6):322-8. [DOI:10.1258/itt.2010.006006] [ISBN:1758-1109 (Electronic)r 1357-633X (Linking)] [PMID:20798426]

4. Tabak M, Brusse-Keizer M, Valk P Van Der, Hermens $H$, Vollenbroek-Hutten M. A telehealth program for self-management of COPD exacerbations and promotion of an active lifestyle: a pilot randomized controlled trial. International journal of COPD. DovePress; 2014. p. 935-44. [DOI:10.2147/COPD.S60179]

5. Evering RMH. Ambulatory Feedback at Daily Physical Activity Patterns: A Treatment for the Chronic Fatigue Syndrome in the Home Environment? University of Twente; 2013. [DOI: 10.3990/1.9789036535120]

6. Petty RE, Cacioppo JT. Issue involvement can increase or decrease persuasion by enhancing message-relevant cognitive responses. J Pers Soc Psychol. 1979;37(10): 1915-26. [DOI: 10.1037/0022-3514.37.10.1915] [ISBN:0022-3514]

7. Bakkes S, Tan CT, Pisan Y. Personalised Gaming: A Motivation and Overview of Literature. Proc 8th Australas Conf Interact Entertain Play Syst '12. 2012;4. [DOI: 10.1 145/2336727.2336731] [ISBN:9781450314107]

8. Vette AFA de, Tabak M, Dekker-van Weering MGH, Vollenbroek-Hutten MMR. Engaging elderly people in telemedicine through gamification. In Press. JMIR Serious Games. [DOl: 10.2196/games.4561]

9. Bartle RA. Hearts, clubs, diamonds, spades: Players who suit MUDs [Internet]. 1996 [cited 2015 Apr 20]. [URL: http://mud.co.uk/richard/hcds.htm Archived at: http://www.webcitation.org/6XvZlirzF]

10. Yee N. Motivations of play in MMORPGs. Proc DiGRA 2005 Conf Chang Views Worlds Play. 2005;

11. Hunicke R, LeBlanc M, Zubek R. MDA: A Formal Approach to Game Design and Game Research. Work Challenges Game Al. 2004; 1-4. [DOI: 10.1.1.79.4561]

12. Gerling KM, Schild J, Masuch M. Exergame design for elderly users: the case study of SilverBalance. Proc 7th Int Conf Adv Comput Entertain Technol - ACE '10. 2010;66-9. [DOI:10.1145/1971630.1971650] [ISBN:9781605588636] [PMID: 15123609 ]

13. Vandenberghe J. The 5 Domains of Play: Applying Psychology's Big 5 Motivation Domains to Games [Internet]. 2012 [cited 2015 Apr 20]. [URL: http://www. gdcvault.com/play/1015364/The-5-Domains-of-Play Archived at: http://www. webcitation.org/6Xva2AWDK]

14. Orii R, Vassileva J, Mandryk RL. Modeling the efficacy of persuasive strategies for different gamer types in serious games for health. User Model User-adapt Interact. 2014; [DOI: 10.1007/s 11 257-014-9149-8]

15. Zammitto VL. Gamers' Personality and their Gaming Preferences. Sch Interact Arts Technol. 2010;M.Sc Thesi: 147. 
16. Teng C. Online Game Player Personality and Real-life Need Fulfillment. Int J Cyber Soc Educ. 2009;2(2):39-50.

17. Zammitto V. Game research, measuring gaming preferences. Appl Artif Intell. 2009;(May):15-6. [DOl:10.1145/1639601.1639611] [ISBN:9781605586854]

18. Peever N, Johnson D, Gardner J. Personality \& video game genre preferences. Proc 8th Australas Conf Interact Entertain Play Syst - IE '12 [Internet]. 2012;13. [URL: http://dl.acm.org/citation.cfm?doid=2336727.2336747] [DOI: 10. 11 145/2336727.2336747] [ISBN:9781450314107]

19. Apperley TH. Genre and game studies: Toward a critical approach to video game genres. Simul Gaming. 2006;37(1):6-23. [DOI: 10.1 177/1046878 105282278]

20. Gosling SD, Rentfrow PJ, Swann WB. A very brief measure of the Big-Five personality domains. J Res Pers. 2003;37(6):504-28. [DOI:10.1016/S00926566(03)00046-1] [ISBN: 1512471593 [PMID:6406]

21. Rammstedt B, John OP. Measuring personality in one minute or less: A 10-item short version of the Big Five Inventory in English and German. J Res Pers. 2007;4 1 (1):20312. [DOI: 10.1016/i.jrp.2006.02.001] [ISBN:0092-6566]

22. Nap HH, Diaz-Orueta U, González MF, Lozar-Manfreda K, Facal D, Dolni ar $V$, et al. Older people's perceptions and experiences of a digital learning game. Gerontechnology [Internet]. 2014;13(3):322-31. [URL: http:// gerontechnology.info/index.php/journal/article/view/2263][DOl: 10.4017/ gt.2015.13.3.002.00]

23. Lucas RE. NIH Public Access. 2009;23(3):558-66. [DOl:10.1037/a0012897. Age]

24. Mroczek DK, Spiro A, Griffin PW. Handbook of the Psychology of Aging [Internet]. Handbook of the Psychology of Aging. Elsevier; 2006 [cited 2015 Aug 4]. 363-377 p. [URL: http://www.sciencedirect.com/science/article/pii/ B9780121012649500197] [DOI:10.1016/B978-012101264-9/50019-7] [ISBN:9780121012649]

\section{Game preferences and personality of older adult users}

1. Deterding S, Dixon D, Khaled R, Nacke L. From game design elements to gamefulness. Proc 15th Int Acad MindTrek Conf Envisioning Futur Media Environ - MindTrek ' 11. 2011 ; 9-11. [DOI:10.1145/2181037.2181040] [ISBN:9781450308168]

2. Evering RMH. Ambulatory Feedback at Daily Physical Activity Patterns: A Treatment for the Chronic Fatigue Syndrome in the Home Environment? University of Twente; 2013. [DOI: 10.3990/1.9789036535120]

3. Gerling K, Masuch M. Exploring the potential of gamification among frail elderly persons. CHI 2011 Work Gamification Using Game Des. 2011;1-4. [ISBN:9781450302685]

4. McCrae RR, Costa PT. The Five-Factor Theory of Personality. In: John OP, editor. Handbook of Personality: Theory and Research. Third Edit. Guilford Press; 2008. [ISBN: 1606237381 ] 
5. McCrae RR, John OP. An introduction to the five-factor model and its applications. J Pers. 1992;60(2): 175-215. [DOl:10.1111/i.1467-6494.1992.tb00970.x] [ISBN:00223506] [PMID: 1635039]

6. Pavel M, Jimison H, Hayes T, Cooper R a, Marottoli R. Technologies for an Aging Population. Bridg Natl Acad Eng. 2009;39(1):68.

7. Tabak M, Brusse-Keizer M, Valk P Van Der, Hermens $H$, Vollenbroek-Hutten M. A telehealth program for self-management of COPD exacerbations and promotion of an active lifestyle: a pilot randomized controlled trial. International journal of COPD. DovePress; 2014. p. 935-44. [DOI:10.2147/COPD.S60179]

8. Vandenberghe J. The 5 Domains of Play: Applying Psychology's Big 5 Motivation Domains to Games [Internet]. 2012 [cited 2015 Apr 20]. [URL: http://www. gdcvault.com/play/1015364/ The-5-Domains-of-Play Archived at: http://www. webcitation.org/6Xva2AWDK]

9. Vette AFA de, Tabak M, Dekker-van Weering MGH, Vollenbroek-Hutten MMR. Engaging elderly people in telemedicine through gamification. In Press. JMIR Serious Games. [DOl: 10.2196/games.4561].

10. Vette AFA de, Tabak M, Dekker-van Weering MGH, Vollenbroek-Hutten MMR. Exploring personality and game preferences: a pilot study. In progress.

\section{Chapter 4| Mapping game preferences of older adults: a field study towards tailored gamified applications}

1. S. Jansen-Kosterink, "The added value of telemedicine services for physical rehabilitation", University of Twente, 2014.

2. Baur, J.Fehr, C. Mayer, C. Pawlu, F. Schaudel, "Health care beyond medicine: Meeting the demand for new forms of care", Health International 11, pp. 56-63, 2011.

3. Przywara, "Projecting future health care expenditure at European level: drivers, methodology and main results", Economic Papers 417, 2010.

4. United Nations, "World Population Prospects: The 2012 Revision", press release, 2012.

5. World Health Organization, "Active Ageing, A Policy Framework", 2002.

6. M. Tabak, M. Brusse-Keizer, P. van der Valk, H. Hermens, M. Vollenbroek-Hutten, "A telehealth program for self-management of COPD exacerbations and promotion of an active lifestyle: a pilot randomized controlled trial", International Journal of COPD, pp. 935-44, 2014.

7. R.M.H. Evering, "Ambulatory Feedback at Daily Physical Activity Patterns: A Treatment for the Chronic Fatigue Syndrome in the Home Environment?", University of Twente, 2013.

8. D.L. Kappen, P. Mirza-Babaei, L.E. Nacke, "Older adults' physical activity and exergames: a systematic review", International Journal of Human-Computer Interaction, 2018. 
9. C. Ramprasad, L. Tamariz, J. Garcia-Barcena, Z. Nemeth, A. Palacio, "The use of tablet technology by older adults in health care settings - Is it effective and satisfying? A systematic review and meta analysis", Clinical Gerontology, June 9: 1-10, 2017.

10. A.K. Hall, E. Chavarria, V. Maneeratana, B.H. Chaney, J.M. Bernhardt, "Health Benefits of Digital Videogames for Older Adults: A Systematic Review of the Literature", Games for Health Journal, Dec; 1(6):402-10, 2012.

11. D. Johnson, S. Deterding, K. Kuhn, A. Staneva, S. Stoyanov, L. Hides, "Gamification for health and wellbeing: a systematic review of the literature", Internet Interventions, Vol. 6, p. 89-106, 2016.

12. T.H. Apperly, "Genre and game studies: Toward a critical approach to video game genres", Simulation and Gaming, 37(6), 6-23, 2006.

13. J. Vandenberghe, "The 5 Domains of Play: Applying Psychology's Big 5 Motivation Domains to Games", 2012.

14. R.R. McCrae RR, P.R. Costa, "The Five-Factor Theory of Personality", In: John OP, editor. Handbook of Personality: Theory and Research, Guilford Press, 2008.

15. N. Peever, "Personality \& Video Game Genre Preferences", IE ' 12 Proceedings of the 8th Australasian conference on Interactive Entertainment, 2012.

16. C. deGraft-Johnson, "Relating Five Factor Personality Traits to Video Game Preference", HCIL Human-Computer Interaction Technical Report, 2013.

17. J.M. Sukeena, A. Moore, M. Minear, "The Relationship Between Video Game Motivations and Personality", Society for Personality and Social Psychology conference, 2013.

18. D. Codish, G. Ravid, "Personality based gamification: how different personalities perceive gamification", Proceedings ECIS, 2014.

19. T.J. Bouchard, J.C. Loehlin, "Genes, Evolution, and Personality" Behavior Genetics, Vol. 31 , No. 3, 2001.

20. M. Gurven, C. Von Rueden, M. Massenkoff, H. Kaplan, M. Lero Vie, "How universal is the big five? Testing the Five-Factor Model of personality variation among foragerfarmers in the Bolivian Amazon" Journal of Personality and Social Psychology, Vol. 104, 2:354-370, 2013.

21. R.R. McCrae, "Cross-Cultural Research on the Five-Factor Model of Personality", Online Readings in Psychology and Culture, 2002.

22. R.R. McCrae, A. Terracciano, "78 members of Personality Profiles of Cultures Project, 2005 - Universal features of personality traits from the observer's perspective: data from 50 cultures" - Journal of Personality and Social Psychology, Vol. 88, 3:547$561,2005$.

23. D.P. Schmitt, J. Allik, R.R. McCrae, V. Benet-Martínez, "The geographic distribution of Big Five Personality traits: Patterns and profiles of human self-description across 56 nations", Journal of Cross-Cultural Psychology, 2007.

24. A.F.A. de Vette, M. Tabak, M.G.H. Dekker-van Weering, M.M.R. Vollenbroek-Hutten, "Exploring personality and game preferences in the younger and older population: a pilot study", Proceedings of ICT4AgeingWell, 2015. 
25. A.F.A. de Vette, M. Tabak, H.J. Hermens, M.M.R. Vollenbroek-Hutten, "Game preferences and personality of older adult users", CHI PLAY conference, 2015.

26. F. de Vette, M. Tabak, M. Dekker-van Weering, M. Vollenbroek-Hutten, "Engaging Elderly People in Telemedicine Through Gamification", JMIR Serious Games, 3(2):e9, 2015.

27. K.M. Gerling, J. Schild, M. Masuch, "Exergame design for elderly users: the case study of SilverBalance", Proc 7th Int Conf Adv Comput Entertain Technol - pp. 6669, 2010.

28. R. Planinc, I. Nake, M. Kampel, "Exergame Design Guidelines for Enhancing Elderly's Physical and Social Activities", Int Conf Ambient Comput Appl Serv Technol pp. 58-63, 2013.

29. W. IJsselsteijn, H.H. Nap, Y. de Kort, K. Poels, "Digital Game Design for Elderly Users", Proc 2007 Conf Futur Play, pp. 17-22, 2007.

30. T. Phiriyapokanon, "Is a big button interface enough for elderly users? Towards user interface Guidelines for Elderly users, Thesis, Mälardalen University Sweden, 2011.

31. M. Csikszentmihalyi, "Flow: the psychology of optimal experience", Acad Manag Rev., HarperPerennial, 1990.

\section{How to design game-based healthcare applications for children? A study on children's game preferences}

1. Jansen-Kosterink, S., 2014. The added value of telemedicine services for physical rehabilitation. University of Twente. [ISBN:97890823 19606] [PMID: 17725187 ]

2. Huis in 't Veld, RMHA., Kosterink, SM., Barbe, T., Lindegård, A., Marecek, T., Vollenbroek-Hutten, MMR., 2010. Relation between patient satisfaction, compliance and the clinical benefit of a teletreatment application for chronic pain. J Telemed Telecare, 2010; 16(6):322-8. [DOI: 10.1258/jtt.2010.006006] [ISBN: 17581109 (Electronic)r 1357-633X (Linking)] [PMID:20798426]

3. Tabak, M., Brusse-Keizer, M., Valk, P. Van Der., Hermens, H., Vollenbroek-Hutten, M., 2014. A telehealth program for self-management of COPD exacerbations and promotion of an active lifestyle: a pilot randomized controlled trial. International journal of COPD. DovePress; 2014. p. 935-44. [DOI: 10.2147/COPD.S60179]

4. Evering, RMH., 2013. Ambulatory Feedback at Daily Physical Activity Patterns: A Treatment for the Chronic Fatigue Syndrome in the Home Environment? University of Twente. [DOI: 10.3990/1.9789036535120]

5. Primack, B., Carroll, M., McNamara, M., Klem, M., King, B., et al. 2012. Role of Video Games in Improving Health-Related Outcomes: A Systematic Review. American Journal of Preventive Medicine 2012 vol: 42 (6) pp: 630-638.

6. Baranowski, T., Buday, R., Thompson, D., Baranowski, J., 2007. Playing for Real: Video Games and Stories for Health-Related Behavior Change. American Journal of Preventive Medicine Volume 34, Issue 1, January 2008, Pages 74-82.e10. [DOI: 10.1016/i.amepre.2007.09.027] 
7. Bartle, RA., 1996. Hearts, clubs, diamonds, spades: Players who suit MUDs. [URL: http://mud.co.uk/richard/hcds.htm Archived at: http://www.webcitation. org/6XvZlirzF]

8. Yee, N., 2015. Motivations of play in MMORPGs. Proc DiGRA Conf Chang Views - Worlds Play 2005.

9. Dixon, D., 2011 . Player Types and Gamification. CHI 2011 Work Gamification Using Game Des Elem NonGame Context. 201 1;12-5. [DOI:ACM 978-45030268-5/11/05] [ISBN:9781450302685]

10. Apperley, TH., 2006. Genre and game studies: Toward a critical approach to video game genres. Simul Gaming. 2006;37(1):6-23. [DOI: 10.1177/1046878105282278]

11. McCrae, R., Costa, P., 2008. The Five-Factor Theory of Personality. In: John OP, editor. Handbook of Personality: Theory and Research, Guilford Press.

12. Vandenberghe, J., 2012. The 5 Domains of Play: Applying Psychology's Big 5 Motivation Domains to Games.

13. De Vette, F., Tabak, M., Hermens, H., Vollenbroek-Hutten, M., 2017. Mapping game preferences of older adults: a field study towards a design model for gamebased applications. In progress.

14. De Vette, A.F.A., Tabak, M. Hermens, H.J., Vollenbroek-Hutten, M.M.R., 2015. Game preferences and personality of older adult users. CHI PLAY conference, London.

15. De Vette, F., Tabak, M., Hermens, H., Vollenbroek-Hutten, M., 2016. Online gaming and training platform against frailty in elderly people. Games for Health conference, Utrecht.

16. Charlier, N., Zupancic, N., Fieuws, S., Denhaerynck, K., Zaman, B., Moons, P., 2016. Serious games for improving knowledge and self-management in young people with chronic conditions: a systematic review and meta-analysis. J Am Med Inform Assoc. 2016;23:230-9. [doi: 10.1093/jamia/ocv100]

17. De Sousa Borges, S., Durelli, V., Macedo Reis, H., Isotani, S., 2014. A systematic mapping on gamification applied to education. Proceedings of the 29th Annual ACM Symposium on Applied Computing, March 24-28, 2014 , Gyeongju, Republic of Korea. [doi: 10.1 145/2554850.2554956]

18. Baranowski, T., Blumberg, F., Buday, R., DeSmet, A., Fiellin, L., Green, C., et al. 2015. Games for Health for Children-Current Status and Needed Research. Games Health J, 5( 1): 1-12. [doi: 10.1089/g4h.2015.0026]

19. De Vette, F., Tabak, M., Dekker-van Weering, M., Vollenbroek-Hutten, M., 2015. Engaging Elderly People in Telemedicine Through Gamification. JMIR Serious Games, 3(2):e9, 2015.

20. Klaassen, R., Van Delden, R,. Cabrita, M., Tabak, M., 2017. AlRPlay: Towards a 'Breathgiving' Approach. Fitfth International Workshop on Behavioral Change Support Systems (BCSS'17), Persuasive Technology 2017.

21. Kafai, Y., 1998. Video game designs by girls and boys: variability and consistency of gender differences. In H. Jenkins, \&J. Cassell (1998) (Eds.), From Barbie to Mortal Kombat (pp. 90-1 17). Cambridge, MA: MIT Press 


\section{Chapter 5 T The 6D Framework: an evidence-based tool for designers of game-based applications}

1. Daniel Johnson, Sebastian Deterding, Kerri-Ann Kuhn, Aleksandra Staneva, Stoyan Stoyanov, Leanne Hides. 2016. Gamification for health and wellbeing: A systematic review of the literature. In Internet Interventions 6 (2016) 89-106. http://dx.doi. org/10.1016/i.invent.2016.10.002

2. Brian A. Primack, Mary V. Carroll, Megan McNamara, Mary Lou Klem, Brandy King, Michael Rich, Chun W. Chan, Smita Nayak. 2012. Role of Video Games in Improving Health-Related Outcomes: A Systematic Review. In American Journal of Preventive Medicine. 42(6):630 -638. https://dx.doi.org/10.1016/i. amepre.2012.02.023

3. Douglas B. Clark, Emily E. Tanner-Smith, Stephen S. Killingsworth. 2016. Digital Games, Design, and Learning: A Systematic Review and Meta-Analysis. In Review of Educational Research March 2016, Vol. 86, No. 1, pp. 79-122. https://doi. org/10.3102\%2F0034654315582065

4. M. Graafland, J.M. Schraagen, M.P. Schijven. 2012. Systematic review of serious games for medical education and surgical skills training. In The British journal of surgery 2012 Oct; 99(10): 1322-30.. https://dx.doi.org/10.1002/bjs.8819

5. Thomas Hainey, Thomas M. Connolly, Elizabeth A. Boyle, Amanda Wilson, Aisya Razak. 2016. A systematic literature review of games-based learning empirical evidence in primary education. In Computers \& Education 102 (2016) 202e223. https://doi.org/10.1 145/1234567890

6. Pedro Pereira, Emília Duarte, Francisco Rebelo, Paulo Noriega. 2014. A Review of Gamification for Health-Related Contexts. In User Experience Design for Diverse Interaction Platforms and Environments. DUXU 2014. Lecture Notes in Computer Science, vol 8518. https://doi.org/10.1007/978-3-319-07626-3_70

7. Lennart Nacke, Sebastian Deterding, 2017. The maturing of gamification research. In Computers in Human Behavior 71 (2017) 450e454. http://dx.doi. org/10.1016/i.chb.2016.11.062

8. Rita Orii, Julita Vassileva, Regan L. Mandryk. 2014. Modeling the efficacy of persuasive strategies for different gamer types in serious games for health. In User Modelling and User-Adapted Interaction. DOI 10.1007/s 1 1257-014-9149-8

9. De Vette AFA, Tabak M, Dekker-van Weering MGH, Vollenbroek-Hutten MMR. Engaging Elderly People in Telemedicine Through Gamification. In JMIR Serious Games, 3(2):e9. 2015

10. Nick Yee. 2007. Motivations for Play in Online Games. In CyberPsychology \& Behavior, Vol. 9, No. 6. https://doi.org/10.1089/cpb.2006.9.772

11. Robin Hunicke, Marc LeBlanc, Robert Zubek. MDA: A Formal Approach to Game Design and Game Research.. In Work Challenges Game Al. 2004; 1-4.

12. Jason Vandenberghe. 2012. The 5 Domains of Play: Applying Psychology's Big 5 Motivation Domains to Games. Retrieved from http://www.gdcvault.com/ play/1015364/The-5-Domains-of-Play Archived at: http://www.webcitation. org/6Xva2AWDK 
13. De Vette AFA, Tabak M, Dekker-van Weering MGH, Vollenbroek-Hutten MMR. Exploring personality and game preferences in the younger and older population: a pilot study. In Proceedings of ICT4AgeingWell (ICT4Awe). 2015

14. De Vette AFA, Tabak M, Hermens HJ, Vollenbroek-Hutten MMR. Mapping game preferences of older adults: a field study towards tailored gamified applications. In Proceedings of NordiCHI' 18. 2018

15. De Vette AFA, Tabak M, Hermens HJ, Vollenbroek-Hutten MMR. How to design gamebased healthcare applications for children? A study on children's game preferences. In Proceedings of the 11 th International Joint Conference on Biomedical Engineering Systems and Technologies, HealthINF 2018

16. Alberto Mora, Daniel Riera, Carina González, Joan Arnedo-Moreno. 2017. Gamification: a systematic review of design frameworks. In Journal of Computing in Higher Education (2017) 29:516-548. https://dx.doi.org/10.1007/s 12528 017-9150-4

17. Sebastian Deterding. 2015. The lens of intrinsic skill atoms: A method for gameful design. In Human-Computer Interaction, 30(3e4), 294e335. http://dx.doi.org/ 1 0.1080/07370024.2014.993471

18. Lamyae Sardi, Ali Idri, José Luis Fernández-Alemán. 2017. A systematic review of gamification in e-Health. In Journal of Biomedical Informatics 71 (2017) 31-48. http://dx.doi.org/10.1016/i.jbi.2017.05.011

19. Katie Seaborn, Deborah I. Fels. 2014. Gamification in theory and action: A survey. In International Journal of Human-Computer Studies 74 (2015) 14-31. http:// dx.doi.org/10.1016/i.ijhcs.2014.09.006

20. J. Kim. 2010. Gamification workshop. Retrieved from http://www.slideshare.net/ amyjokim/gamification-workshop-2010

21. Kevin Werbach, Dan Hunter. 2012 . For the win: How game thinking can revolutionize your business. Philadelphia, PA: Wharton Digital Press. ISBN 978-1613630235

22. Gabe Zichermann, Christopher Cunningham. 2011. Gamification by design: Implementing game mechanics in web and mobile apps. Sebastopol, CA: O'Reilly. ISBN 9781449397678

23. Karl Kapp. 2012. The gamification of learning and instruction: Game-based methods and strategies for training and education. San Francisco, CA: Pfeiffer. ISBN: 9781118096345

24. Biran Burke. 2014. Gamify: How gamification motivates people to do extraordinary things. Brookline, MA: Bibliomotion. ISBN 978-1937134853

25. Richard Bartle. 2011. Gamification: Too much of a good thing? Retrieved from http://www.mud.co.uk/richard/Shoreditch.pdf

26. Jesse Schell. 2009. The art of game design: A book of lenses. Morgan Kaufman. ISBN 9780123694966

27. Margaret Robertson. 2010. Can't play, won't play. In Blog: Hide\&Seek. Retrieved from http://hideandseek.net/2010/10/06/cant-play-wont-play Archived at http://www.webcitation.org/6XvYdsA2p 
28. Ian Bogost. 2011. Gamification is Bullshit. Retrieved from hitp://bogost.com/ writing/blog/gamification_is_bullshit/ Archived at: http://www.webcitation. org/6XVZOUBql

29. Andrés Francisco Aparicio, Francisco Luis Gutiérrez Vela, José Luis González Sánchez, José Luis Isla Montes. 2012. Analysis and application of gamification. In Proceedings of the 13th International Conference on Interacción Persona-Ordenador. https://doi.org/10.1145/2379636.2379653

30. Scott Nicholson. 2012. A user-centered theoretical framework for meaningful gamification. In C. Martin, A. Ochsner, \& K. Squire (Eds.), GLS 8.0 (pp. 223-230).

31. Jorge Simões, Rebeca Díaz Redondo, Ana Fernández Vilas. 2013. A social gamification framework for a K-6 learning platform. In Computers in Human Behavior, 29, 345-353. https://doi.org/10.1016/i.chb.2012.06.007

32. Mizuki Sakamoto, Tatsuo Nakajima, Todorka Alexandrova. 2012. Value-Based Design for Gamifying Daily Activities. In Herrlich M., Malaka R., Masuch M. (eds) Entertainment Computing - ICEC 2012. ICEC 2012. Lecture Notes in Computer Science, vol 7522. hitps://doi.org/10.1007/978-3-642-33542-6_43

33. Deborah Gears, Karen Braun. 2013. Gamification in business: Designing motivating solutions to problem situations. In Proceedings of the $\mathrm{CHI} 2013$ workshop Designing Gamification. Retrieved from hitp://gamification-research.org/wp-content/ uploads/2013/03/Gears_Braun.pdf

34. Dan Dixon. 2011 . Player Types and Gamification. In Proceedings of ACM CHI 201 1, May 7-1 2201 1, Vancouver, Canada. ACM 978-1-4503-0268-5/1 1/05

35. Dominic Arsenault. 2009. Video Game Genre, Evolution and Innovation. In Eludamos. Journal for Computer Game Culture. 2009; 3 (2), p. 149-176.

36. Jin Ha Lee, Natascha Karlova, Rachel Ivy Clarke, Katherine Thornton, Andrew Perti. 2014. Facet Analysis of Video Game Genres. In iConference 2014 Proceedings (p. 125-139). doi: 10.9776/14057

37. Veronica Zammitto. 2009. Game research, measuring gaming preferences. In Appl Artif Intell. 2009;(May):15-6. hitps://doi.org/10.1 145/1639601.1639611

38. Ernest Adams, Barry lp. 2002. From casual to core: a statistical mechanism for studying gamer dedication. Retrieved from www.gamasutra.com/view/feature/131397/ from_casual_to_core_a_statistical_.php

39. C.H. Lawshe. 1975. A Quantitative Approach to Content Validity. In Personnel Psychology, 28, 563-575. hitp://dx.doi.org/10.1111//.1744-6570.1975. tb01393.x

40. Gregory E. Gilbert, Susan Prion. 2016. Making Sense of Methods and Measurement: Lawshe's Content Validity Index. In International Nursing Association for Clinical Simulation and learning. http://dx.doi.org/10.1016/i.ecns.2016.08.002

41. Mihaly Csikszentmihalyi. 1975. Beyond Boredom and Anxiety. Jossey-Bass Publishers, 2000.

42. Marc-André K. Lafrenière, Jérémie Verner-Filion, Robert J. Vallerand. 2012. Development and validation of the Gaming Motivation Scale (GAMS). In Personality and Individual Differences 53 (2012) 827-831. http://dx.doi.org/10.1016/i. paid.2012.06.013 
43. Marco Pasch, Nadia Bianchi-Berthouze, Betsy van Dijk, Anton Nijholt. 2009. Movement-based sports video games: Investigating motivation and gaming experience. In Entertainment Computing 1 (2009) 49-61. http://dx.doi. org/10.1016/i.entcom.2009.09.004

44. Richard M. Ryan, C. Scott Rigby, Andrew Przybylski. 2006. The Motivational Pull of Video Games: A Self-Determination Theory Approach. In Motivation \& Emotion. Dec2006, Vol. 30 Issue 4, p344-360. http://dx.doi.org/10.1007/s 11031 006-905 1-8

45. Fan-Chen Tseng. 2011 . Segmenting online gamers by motivation. In Expert Systems with Applications 38 (2011) 7693-7697. http://dx.doi.org/10.1016/i. eswa.2010.12.142

46. Chia-chen Yang, Dong Liu. 2017. Motives Matter: Motives for Playing Pokémon Go and Implications for Well-Being. In CyberPsychology, Behavior \& Social Networking. Jan2017, Vol. 20 Issue 1, p52-57. 6p. http://dx.doi.org/10.1089/ cyber.2016.0562

47. Tim Wulf, Nicholas David Bowman, Diana Rieger, John A. Velez, Johannes Breuer. 2018 . Video Games as Time Machines: Video Game Nostalgia and the Success of Retro Gaming. In Media and Communication (ISSN: 2183-2439) 2018, Volume 6, Issue 2, Pages 60-68s. http://dx.doi.org/10.17645/mac.v6i2.1317

48. Sebastian Deterding. 2012. Gamification: designing for motivation. In Magazine interactions. Volume 19 issue 4, July August 2012, pages 14-17. ACM New York. http://dx.doi.org/10.1145/2212877.2212883

49. Juho Hamari, Jonna Koivisto. 2013. Social Motivations To Use Gamification: An Empirical Study Of Gamifying Exercise. In Proceedings of the 21 st European Conference on Information Systems. http://dx.doi.org/10.1145/1234567890

\section{Chapter 6| A gamified physical activity coaching application for older adults: design approach and user experience in daily life}

1. $\mathrm{WHO}$, Global recommendations on physical activity for health, 2012.

2. Azagba, S and Sharaf, MF, Physical inactivity among older Canadian adults. J Phys Act Health, 2014. 11 (1): p. 99-108.

3. Townsend, N, Wickranmasinghe, K, Williams, J, Bhatnagar, P, and Rayner, M, Physical activity statistics 2015, 2015, British Heart Foundation.

4. Clemes, SA, O'Connell, SE, and Edwardson, CL, Office workers' objectively measured sedentary behavior and physical activity during and outside working hours. J Occup Environ Med, 2014. 56(3): p. 298-303.

5. Kohl, LF, Crutzen, R, and de Vries, NK, Online prevention aimed at lifestyle behaviors: a systematic review of reviews. J Med Internet Res, 2013. 15(7): p. el46.

6. Tabak, M, Brusse-Keizer, M, van der Valk, P, Hermens, H, and Vollenbroek-Hutten, $M$, A telehealth program for self-management of COPD exacerbations and promotion of an active lifestyle: a pilot randomized controlled trial. Int J Chron Obstruct Pulmon Dis, 2014. 9: p. 935-44. 
7. Brouwer, W, Kroeze, W, Crutzen, R, de Nooijer, J, de Vries, NK, Brug, J, and Oenema, A, Which intervention characteristics are related to more exposure to internet-delivered healthy lifestyle promotion interventions? A systematic review. J Med Internet Res, $2011.13(1)$ : p. e2.

8. Davies, CA, Spence, JC, Vandelanotte, C, Caperchione, CM, and Mummery, WK, Meta-analysis of internet-delivered interventions to increase physical activity levels. Int J Behav Nutr Phys Act, 2012. 9: p. 52.

9. Vandelanotte, C, Spathonis, KM, Eakin, EG, and Owen, N, Website-delivered physical activity interventions a review of the literature. Am J Prev Med, 2007. 33(1): p. 54-64.

10. Sabate, E, Adherence to long-term therapies. Evidence for action., 2003, World Health Organization.

11. Crutzen, R, de Nooijer, J, Brouwer, W, Oenema, A, Brug, J, and de Vries, NK, Strategies to facilitate exposure to internet-delivered health behavior change interventions aimed at adolescents or young adults: a systematic review. Health Educ Behav, 2011 . 38(1): p. 49-62.

12. Haynes, RB, McKibbon, KA, and Kanani, R, Systematic review of randomised trials of interventions to assist patients to follow prescriptions for medications. Lancet, 1996. 348(9024): p. 383-6.

13. van den Berg, N, Schumann, M, Kraft, K, and Hoffmann, W, Telemedicine and telecare for older patients-a systematic review. Maturitas, $2012.73(2)$ : p. 94-1 14.

14. Dennison, L, Morrison, L, Lloyd, S, Phillips, D, Stuart, B, Williams, S, Bradbury, K, Roderick, P, Murray, E, Michie, S, Little, P, and Yardley, L, Does brief telephone support improve engagement with a web-based weight management intervention? Randomized controlled trial. J Med Internet Res, 2014. 16(3): p. e95.

15. Tabak, $M$, Dekker-van Weering, $M$, van Dijk, $H$, and Vollenbroek-Hutten, $M$, Promoting Daily Physical Activity by Means of Mobile Gaming: A Review of the State of the Art. Games Health J, 2015. 4(6): p. 460-9.

16. de Vette, F, Tabak, M, Dekker-van Weering, M, and Vollenbroek-Hutten, M, Engaging Elderly People in Telemedicine Through Gamification. JMIR Serious Games, 2015. 3(2): p. e9.

17. Lewis, ZH, Swartz, MC, and Lyons, EJ, What's the Point?: A Review of Reward Systems Implemented in Gamification Interventions. Games Health J, 2016. 5(2): p. 93-9.

18. Deci, EL, Koestner, R, and Ryan, RM, A meta-analytic review of experiments examining the effects of extrinsic rewards on intrinsic motivation. Psychol Bull, 1999. 125(6): p. 627-68; discussion 692-700.

19. Yardley, L, Spring, BJ, Riper, H, Morrison, LG, Crane, DH, Curtis, K, Merchant, GC, Naughton, F, and Blandford, A, Understanding and Promoting Effective Engagement With Digital Behavior Change Interventions. Am J Prev Med, 2016. 51 (5): p. 833842.

20. Brown, M, O'Neill, N, van Woerden, H, Eslambolchilar, P, Jones, M, and John, A, Gamification and Adherence to Web-Based Mental Health Interventions: A Systematic Review. JMIR Ment Health, 2016. 3(3): p. e39. 
21. Op den Akker, H, Tabak, M, Marin-Perianu, M, Huis in't Veld, R, Jones, VM, Hofs, D, Tönis, TM, van Schooten, BW, Vollenbroek-Hutten, MMR, and Hermens, HJ, Development and Evaluation of a Sensor-Based System for Remote Monitoring and Treatment of Chronic Diseases - the Continuous Care \& Coaching Platform. Proceedings of EHST 2012: the 6th International Symposium on eHealth Services and Technologies, Geneva, Switzerland, July 2012.

22. Dekker-van Weering, MG, Vollenbroek-Hutten, MM, and Hermens, HJ, A pilot study - the potential value of an activity-based feedback system for treatment of individuals with chronic lower back pain. Disabil Rehabil, 2015. 37(24): p. 2250-6.

23. Bruggeman-Everts, FZ, Wolvers, MDJ, van de Schoot, R, Vollenbroek-Hutten, MMR, and Van der Lee, ML, Effectiveness of Two Web-Based Interventions for Chronic Cancer-Related Fatigue Compared to an Active Control Condition: Results of the "Fitter na kanker" Randomized Controlled Trial. J Med Internet Res, 2017. 19(10): p. e336.

24. Tabak, $M$, op den Akker, $H$, and Hermens, $H$, Motivational cues as real-time feedback for changing daily activity behavior of patients with COPD. Patient Educ Couns, 2014. 94(3): p. 372-8.

25. De Vette, A, Tabak, M, Hermens, H, and Vollenbroek-Hutten, M, Mapping Game Preferences of Older Adults: A Field Study Towards Tailored Gamified Applications, in NordiCHI 20182018, ACM: Oslo, Norway.

26. Spook, J, Paulussen, T, Kok, G, and van Empelen, P, Evaluation of a Serious SelfRegulation Game Intervention for Overweight-Related Behaviors ("Balance It"): A Pilot Study. J Med Internet Res, 2016. 18(9): p. e225.

27. Bird, ML, Clark, B, Millar, J, Whetton, S, and Smith, S, Exposure to "Exergames" Increases Older Adults' Perception of the Usefulness of Technology for Improving Health and Physical Activity: A Pilot Study. JMIR Serious Games, 2015. 3(2): p. e8.

28. De Vette, A, Tabak, M, and Vollenbroek-Hutten, $M$, The 6D framework: an evidencebased tool for designers of serious games. Submitted, 2018.

29. Kappen, D, Nacke, L, Gerling, K, and Tsotsos, L, Design strategies for gamified physical activity applications for older adults, in 2016 49th Hawaii International Conference on System Sciences2016.

30. De Schutter, B, Never Too Old to Play: The Appeal of Digital Games to an Older Audience. Games and Culture, 2011 . 6(2): p. 155-170.

31. Muller, I, Rowsell, A, Stuart, B, Hayter, V, Little, P, Ganahl, K, Muller, G, Doyle, G, Chang, P, Lyles, CR, Nutbeam, D, and Yardley, L, Effects on Engagement and Health Literacy Outcomes of Web-Based Materials Promoting Physical Activity in People With Diabetes: An International Randomized Trial. J Med Internet Res, 2017. 19(1): p. e21.

32. Mohr, DC, Cuijpers, $P$, and Lehman, K, Supportive accountability: a model for providing human support to enhance adherence to eHealth interventions. J Med Internet Res, 2011 . 1311): p. e30.

33. Vermeire, E, Hearnshaw, H, Van Royen, P, and Denekens, J, Patient adherence to treatment: three decades of research. A comprehensive review. J Clin Pharm Ther, 2001. 26(5): p. $331-42$. 
34. Buimer, HP, Tabak, M, van Velsen, L, van der Geest, T, and Hermens, H, Exploring Determinants of Patient Adherence to a Portal-Supported Oncology Rehabilitation Program: Interview and Data Log Analyses. JMIR Rehabil Assist Technol, 2017. 4(2): p. el2.

35. Perski, O, Blandford, A, West, R, and Michie, S, Conceptualising engagement with digital behaviour change interventions: a systematic review using principles from critical interpretive synthesis. Transl Behav Med, 2017. 7(2): p. 254-267.

\section{Chapter 7| Game-based design for eHealth in practice}

1. Alpay L, van der Boog P, Dumaij A. An empowerment-based approach to developing innovative e-health tools for self-management. Health informatics journal. 2011 Dec; 17(4): p. 247-55. doi: 10.1177/1460458211420089

2. Free C, Phillips G, Galli L, Watson L, Felix L, Edwards P, et al. The effectiveness of mobile-based health technology-based health behaviour change or disease management interventions for health care consumers: a systematic review. PLoS medicine. 2013 Jan 15;10(1): p. e1001362. https://doi.org/10.1371//ournal. pmed. 1001362

3. Jacobs RJ, Lou JQ, Ownby RL, Caballero J. A systematic review of eHealth interventions to improve health literacy. Health Informatics Journal. 2016 22(2), $81-98$. https:// doi.org/10.1177/1460458214534092

4. Hamine S, Gerth-Guyette E, Faulx D, Green BB, Ginsburg AS. Impact of mHealth chronic disease management on treatment adherence and patient outcomes: a systematic review. Journal of medical Internet research. 2015, 17(2), e52. doi: 10.2196/jmir.3951

5. Carels RA, Selensky JC, Rossi J, Solar C, Hlavka R. A novel stepped-care approach to weight loss: The role of self-monitoring and health literacy in treatment outcomes. Eating Behaviors, Volume 26, 2017, Pages 76-82, ISSN 1471-0153, https://doi. org/10.1016/i.eatbeh.2017.01.009.

6. Eysenbach G. What is e-health? J Med Internet Res 200 1;3(2):e20. DOI: 10.2196/ imir.3.2.e20

7. Huis in 't Veld RMHA, Kosterink SM, Barbe T, Lindegård A, Marecek T, VollenbroekHutten MMR. Relation between patient satisfaction, compliance and the clinical benefit of a teletreatment application for chronic pain. J Telemed Telecare. 2010; 16(6):322-8. DOI: 10.1258/jtt.2010.006006

8. Dekker-van Weering MGH, Vollenbroek-Hutten MMR, Hermens HJ. Adherence to an online exercise program for COPD patients in the home environment- a pilot study. Health Technol. (2016) 6: 259. https://doi.org/10.1007/s 12553-0 16-0137-3

9. Tabak M, Brusse-Keizer M, Valk P Van Der, Hermens H, Vollenbroek-Hutten M. A telehealth program for self-management of COPD exacerbations and promotion of an active lifestyle: a pilot randomized controlled trial. International journal of COPD. DovePress; 2014. p. 935-44. DOI:10.2147/COPD.S60179 
10. Shirong LA, Kharrazi H. A State-of-the-Art Systematic Content Analysis of Games for Health. Games for Health Journal. February 2018, 7(1): 1-15. https://doi. org/10.1089/g4h.2017.0095

11. Sardi L, Idri A, Fernández-Alemán JL. A systematic review of gamification in e-Health. In Journal of Biomedical Informatics 71 (2017) 31-48. http://dx.doi. org/10.1016/i.jbi.2017.05.011

12. Pereira P, Duarte E, Rebelo F, Noriega P. A Review of Gamification for HealthRelated Contexts. In User Experience Design for Diverse Interaction Platforms and Environments. DUXU 2014. Lecture Notes in Computer Science, vol 8518 . https:// doi.org/10.1007/978-3-319-07626-3_70

13. Lange B, Flynn SM, Rizzo AA. Game-based telerehabilitation. Eur J Phys Rehabil Med. 2009;45: 143-51. PMID 19282807

14. Baranowski T, Buday R, Thompson DI, Baranowski J. Playing for real: video games and stories for health-related behavior change. Am J Prev Med. 2008;34:74-82. doi: 10.1016/i.amepre.2007.09.027

15. Seaborn K, Fels DI. Gamification in theory and action: A survey. In International Journal of Human-Computer Studies 74, 2015;14-31. http://dx.doi.org/10.1016/i. ijhcs. 2014.09 .006

16. Johnson D, Deterding S, Kuhn K, Staneva A, Stoyanov S, Hides L. Gamification for health and wellbeing: A systematic review of the literature. In Internet Interventions 6. 2016;89-106. http://dx.doi.org/10.1016/j.invent.2016.10.002

17. Lister C, West JH, Cannon B, Sax T, Brodegard D. Just a Fad? Gamification in Health and Fitness Apps, JMIR Serious Games, 2(2), 2014, pp. e9. doi: 10.2196/ games. 3413

18. Thompson D. Designing serious video games for health behavior change: current status and future directions. Journal of diabetes science and technology 2012;6(4), 807-1 1. doi:10.1177/193229681200600411

19. Kharrazi H, Shirong LA, Gharghabi F, Coleman W. A Scoping Review of Health Game Research: Past, Present, and Future Games for Health Journal. April 2012, 1(2): 153-164. https://doi.org/10.1089/g4h.2012.0011

20. Lu AS, Kharrazi H, Gharghabi F, Thompson D. A Systematic Review of Health Videogames on Childhood Obesity Prevention and Intervention. Games for health journal, 2013;2(3), 131-141. DOI: 10.1089/g4h.2013.0025

21. Laxman K, Krishnan SB, Dhillon JS. Barriers to Adoption of Consumer Health Informatics Applications for Health Self Management. January 2015 Health Science Journal 9(5:7): 1-7.

22. Perrotta C, Featherstone G, Aston H, Houghton E. Game-based Learning: Latest Evidence and Future Directions (NFER Research Programme: Innovation in Education). Slough: NFER. Published in April 2013. National Foundation for Educational Research, The Mere, Upton Park, Slough, Berkshire, SL1 2DQ. ISBN 978-1908666-60-4.

23. Horn B, Hoover AK, Barnes J, Folajimi Y, Smith G, Harteveld C. Opening the Black Box of Play: Strategy Analysis of an Educational Game. In Proceedings of the 2016 Annual Symposium on ComputerHuman Interaction in Play. ACM, 142-153. 
24. Hamari J. Transforming Homo Economicus into Homo Ludens: A Field Experiment on Gamification in a Utilitarian Peer-to-Peer Trading Service. Electronic Commerce Research and Applications, 12(4), 2013, pp. 236-245. DOl:10.1016/i. elerap.2013.01.004

25. Zichermann G, Cunningham C. Gamification by design: Implementing game mechanics in web and mobile apps. 2011. Sebastopol, CA: O'Reilly. ISBN 9781449397678

26. Mora A, Riera D, González C, Arnedo-Moreno J. Gamification: a systematic review of design frameworks. In Journal of Computing in Higher Education. 2017;29:516548. https://dx.doi.org/10.1007/s 12528-017-9150-4

27. Kaleva JP, Hiltunen K, Latva S. Mapping the full potential of the emerging health game markets, (Neogames Finland ry.), September 2013. Retrieved from https:// www.neogames.fi/en/industry-info/publications

28. Deterding S, Dixon D, Khaled R, Nacke L. From game design elements to gamefulness. Proc 15th Int Acad MindTrek Conf Envisioning Futur Media Environ - MindTrek '1 1. $2011 ; 9-11$. DOI:10.1145/2181037.2181040

29. Cordova DI, Lepper MR. Intrinsic motivation and the process of learning: Beneficial effects of contextualization, personalization, and choice. J Educ Psychol. 1996;88(4):715-30. DOI: 10.1037/0022-0663.88.4.715. ISBN: 1939-2176 (Electronic); $0022-0663$ (Print)

30. Hamari J, Koivisto J, Pakkanen T. Do Persuasive Technologies Persuade? - A Review of Empirical Studies. In: Spagnolli A., Chittaro L., Gamberini L. (eds) Persuasive Technology. PERSUASIVE 2014. Lecture Notes in Computer Science, vol 8462. https://doi.org/10.1007/978-3-319-07127-5_11

31. De Vette AFA, Tabak M, Dekker-van Weering MGH, Vollenbroek-Hutten MMR. Engaging Elderly People in Telemedicine Through Gamification. 2015(a). In JMIR Serious Games, 3(2):e9.

32. European Innovation Partnership on Active and Healthy Ageing, Action Plan on Prevention and Early Diagnosis of Frailty and Functional Decline, Both Physical and Cognitive in Older People, European Union, Brussels, Belgium, 2012.

33. Sternberg SA, Schwartz AW, Karunananthan S, Bergman H, Mark Clarfield A. The identification of frailty: a systematic literature review. 2011 . Journal of the American Geriatrics Society, 59(11), 21 29-2138.

34. Gomez MI, García-Sánchez I, Carta A, Antunes JP. A Collection of Good Practices That Support the Prevention and Early Diagnosis of Frailty and Functional Decline, Both Physically and Cognitive, in Older People. European Commission, Brussels, Belgium, 2013.

35. Vellas B, Sourdet S: Prevention of Frailty in Aging. The Journal of frailty \& aging 2017, 6(4): 174-177.

36. Van Velsen L, Illario M, Jansen-Kosterink S, et al. A Community-Based, TechnologySupported Health Service for Detecting and Preventing Frailty among Older Adults: A Participatory Design Development Process. Journal of Aging Research, vol. 2015, Article ID 216084, 9 pages, 2015. https://doi.org/10.1 155/2015/216084 
37. O' Caoimh R. et al. The Community Assessment of Risk and Treatment Strategies (CARTS): An Integrated Care Pathway to Manage Frailty and Functional Decline in Community Dwelling Older Adults. In: Helfert M., Holzinger A., Ziefle M., Fred A., O'Donoghue J., Röcker C. (eds) Information and Communication Technologies for Ageing Well and e-Health. ICT4AWE 2015. Communications in Computer and Information Science, vol 578. Springer, Cham. https://doi.org/10.1007/978-33 19-27695-3_1

38. De Vette AFA, Tabak M, Dekker-van Weering MGH, Vollenbroek-Hutten MMR. Exploring personality and game preferences in the younger and older population: a pilot study. 2015(b). In Proceedings of ICT4AgeingWell (ICT4Awe 2015)

39. De Vette AFA, Tabak M, Vollenbroek-Hutten MMR. How to design game-based healthcare applications for children? A study on children's game preferences. 2018. In Proceedings of the $11^{\text {th }}$ International Joint Conference on Biomedical Engineering Systems and Technologies, Volume 5: HEALTHINF. http://dx.doi. org/10.5220/0006584804220430 - 2018a

40. De Vette AFA, Tabak M, Hermens HJ, Vollenbroek-Hutten MMR. Mapping game preferences of older adults: a field study towards tailored gamified applications. 2018. In Proceedings of NordiCHI'18. https://doi.org/10.1 145/3240167.3240204 - 2018b

41. Bates B. Game design. 2014. ISBN 1-59200-493-8, Thomson Course Technology.

42. Nielsen, Jakob, and Landaver, Thomas K.: "A mathematical model of the finding of usability problems," Proceedings of ACM INTERCHI'93 Conference (Amsterdam, The Netherlands, 24-29 April 19931, pp. 206-213.

43. Venkatesh, V., Morris, M., Davis, G., \& Davis, F. (2003). User Acceptance of Information Technology: Toward a Unified View. MIS Quarterly, 27(3), 425-478. doi: $10.2307 / 30036540$

44. Becker SA. A Study of Web Usability for Older Adults Seeking Online Health Resources Transactions on Computer-Human Interaction, Vol. 11, No. 4, December 2004.

45. Chadwick-Dias A, McNulty M, Tullis T. Web Usability and Age: How Design Changes Can Improve Performance. CUU'O3, November 10-1 1, 2003, Vancouver.

46. Planinc R, Nake I, Kampel M. Exergame Design Guidelines for Enhancing Elderly's Physical and Social Activities. Int Conf Ambient Comput Appl Serv Technol pp. 5863, 2013

47. IJsselsteijn W, Nap HH, De Kort Y, Poels K. Digital Game Design for Elderly Users. Proc 2007 Conf Futur Play, pp. 17-22, 2007.

48. Phiriyapokanon T. Is a big button interface enough for elderly users? Towards user interface Guidelines for Elderly users. Thesis, Mälardalen University Sweden, 2011.

49. Isaias P, Issa T. Information System Development Life Cycle Models. High Leve Models and Methodologies for Information Systems. 2015. Springer, New York, NY

50. Aveson D., Fitzgerald G. (2006) Methodologies for Developing Information Systems: A Historical Perspective. In: Avison D., Elliot S., Krogstie J., Pries-Heje J. (eds) The Past and Future of Information Systems: 1976-2006 and Beyond. IFIP WCC TC8 2006. IFIP 
51. Van Gemert-Pijnen JE, Niiland N, van Limburg M, Ossebaard HC, Kelders SM, Eysenbach G, Seydel ER. A Holistic Framework to Improve the Uptake and Impact of eHealth Technologies - J Med Internet Res 201 1;13(4):e 111 International Federation for Information Processing, vol 214. Springer, Boston. DOI: 10.2196/imir. 1672

52. Hussain T, Feurzeig W, Cannon-Bowers J, Coleman S, Koenig A, Lee J, Menaker E, Moffitt K, Murphy C, Pounds K, Roberts B, SeipJ, Souders V, Wainess R. Development of Game-Based Training Systems: Lessons Learned in an Inter-Disciplinary Field in the Making. Source Title: Serious Game Design and Development: Technologies for Training and learning. 2010. DOI: 10.4018/978-1-61520-739-8.ch004

53. Prochaska JO. Transtheoretical Model of Behavior Change. 2013. In: Gellman M.D., Turner J.R. (eds) Encyclopedia of Behavioral Medicine. Springer, New York, NY

\section{Chapter 8| General discussion}

1. Nap HH, De Kort YAW, IJsselsteijn WA. Senior gamers: preferences, motivations and needs. Gerontechnology, Vol. 8, No. 4, p.247-262. ISSN 1569-1 11 1X. 2009

2. De Schutter B, Malliet $\mathrm{S}$. The older player of digital games: A classification based on perceived need satisfaction. Communications, 39/1), pp. 67-87. 2014

3. Skalsky Brown JA. Let's Play: Understanding the Role and Significance of Digital Gaming in Old Age. Theses and Dissertations-Gerontology. 6. 2014

4. Isselsteijn W, Nap HH, De Kort Y, Poels K. Digital Game Design for Elderly Users. Proc 2007 Conf Futur Play, pp. 17-22, 2007

5. Melenhorst AS. Adopting communication technology in later life : the decisive role of benefits Eindhoven: Technische Universiteit Eindhoven DOI: 10.6100/IR/558975. 2002

6. Renaud K, Van Biljon, J. Predicting technology acceptance and adoption by the elderly: a qualitative study. In Proceedings of the 2008 annual research conference of the South African Institute of Computer Scientists and Information Technologists on IT research in developing countries: riding the wave of technology (SAICSIT '08). ACM, New York, NY, USA, 210-219. DOl=http://dx.doi. org/10.1145/1456659.1456684

7. Fisk A, Czaja S, Rogers W, Charness N, Czaja S, Sharit J. Designing for Older Adults. Boca Raton: CRC Press. https://doi.org/10.1201/9781420080681. 2009

8. Roy JSS, Neumann WP, Fels DI. User Centered Design Methods and Their Application in Older Adult Community. In: Yamamoto S. (eds) Human Interface and the Management of Information: Information, Design and Interaction. HIMI 2016. Lecture Notes in Computer Science, vol 9734. Springer

9. Ballegaard SA, Hansen TR, Kyng M. Healthcare in everyday life: designing healthcare services for daily life. In Proceedings of the SIGCHI Conference on Human Factors in Computing Systems (CHI '08). 2008. https://doi. org/10.1145/1357054.1357336 
10. Carmichael A, Rice M, MacMillan F, Kirk A. Investigating a DTV-based physical activity application to facilitate wellbeing in older adults, in: Proceedings of the 24th BCS Interaction Specialist Group Conference. British Computer Society, pp. 278288. 2010

11. Vaziri DD. Facilitating Daily Life Integration of Technologies for Active and Healthy Aging. Informationsmanagement in Theorie und Praxis. 2018

12. Birren JE, Warner Schaie K, editors. Handbook of the Psychology of Aging. 6th edition. New York: Elsevier 2006; doi 10.1080/03601270701328698

13. Gerling KM, Schulte FP, Smeddinck J, Masuch M. Game Design for Older Adults: Effects of Age-Related Changes on Structural Elements of Digital Games. In: Herrlich M, Malaka R, Masuch M (eds) Entertainment Computing. ICEC 2012. Lecture Notes in Computer Science, vol 7522. 2012

14. Marston HR. Digital Gaming Perspectives of Older Adults: Content vs. Interaction, Educational Gerontology, 39:3, 194-208, 2013. DOl: 10.1080/03601277.2012.700817

15. Van Deursen AJAM, Helsper EJ. A nuanced understanding of Internet use and non-use amongst older adults. European Journal of Communication, 30(2), 171-187. 2015

16. Beldad AD, Hegner SM. Expanding the Technology Acceptance Model with the Inclusion of Trust, Social Influence, and Health Valuation to Determine the Predictors of German Users' Willingness to Continue using a Fitness App: A Structural Equation Modeling Approach, International Journal of Human-Computer Interaction, 34:9, 882-893, 2018. DOI: 10.1080/10447318.2017.1403220

17. Cabrita M, Tabak M, Vollenbroek-Hutten MMR. Older Adults' Attitudes Toward Ambulatory Technology to Support Monitoring and Coaching of Healthy Behaviors: Qualitative Study. JMIR Aging, 2(1), [e 10476]. 2019. https://doi. org/10.2196/10476

18. Buimer HP, Tabak M, van Velsen L, van der Geest $T$, and Hermens $H$. Exploring Determinants of Patient Adherence to a Portal-Supported Oncology Rehabilitation Program: Interview and Data Log Analyses. JMIR Rehabil Assist Technol, 2017

19. Jansen-Kosterink S, Vollenbroek-Hutten MM, Hermens HJ. A renewed framework for the evaluation of telemedicine. The Eighth International Conference on eHealth, Telemedicine, and Social Medicine eTELEMED. 2016

20. Broens THF, Huis in't Veld RMHA, Vollenbroek-Hutten MMR, Hermens HJ, van Halteren AT, Nieuwenhuis LIM. Determinants of successful telemedicine implementations: a literature study. Journal of Telemedicine and Telecare, 13(6), 303-309. 2007. https://doi.org/10.1258/135763307781644951 


\section{Summary}

Digital healthcare applications, also called 'eHealth, can contribute to increasing the quality of healthcare and the reduction of the demand for care. eHealth enables remote monitoring and treatment, and offers patients more grip on their own health through deploying self-management applications and improved accessibility of services. A rapidly growing target group that can particularly benefit from eHealth is the older adult (65 years and up). For this group, eHealth can contribute to prolonged autonomy and independence by providing support in adopting and maintaining a healthy lifestyle. It is of vital importance that these eHealth applications are used as they are intended: usually frequent and for a longer period of time. This so-called 'adherence' leaves room for improvement, so that users can optimally benefit from the interventions offered. Games, or specific game elements, can be used in applications that have a primary goal other than entertainment for their natural power to engage people. Specific messages or goals are captured in a game ('serious games') or selected elements from games are added to existing applications ('gamification') in an effort to encourage proper use. This strategy, to which in this thesis we refer to with the umbrella term 'game-based design', has not remained unnoticed in developments in healthcare. After all, there is a lot to gain from effective motivational strategies when goals require tasks that are not rarely boring, repetitive and straining.

This thesis describes how to deploy the power of game-based design to engage users in productive activities, thereby meeting the demand for strategies to increase and improve the use of eHealth applications. The focus is mainly, but not exclusively, to address the older population. Chapter 1 elaborates the background of both eHealth and game-based design in more detail.

In first study of this thesis, described in chapter 2, the status quo of the concept gamification was researched. Immediate cause for this research was the rapid increase in number of initiatives using gamification from a variety of backgrounds and research areas, but the lack of consensus on the concept itself. How to approach gamification to realise long-term engagement appeared to be an underexplored topic, particularly for the older population: methods to apply gamebased design, incorporating a solid theoretical foundation as well as practical guidelines, are not readily available. In this study, a broad view on gamification activities was taken within as well as outside of academic research, such as the gaming industry and business. An understanding of the theoretical background of gamification was provided and insight was gained in existing conceptual models ('frameworks') to develop gamification, for the general or older audience. Existing user classifications that may be used to tailor game design to specific users were 
thereby explored. The results showed that two main approaches emerged in the conceptualisation of gamification and the definitions of related concepts, such as game elements. On one hand there is gamification applied in corporate, marketing and customer engagement context; on the other hand gamification not focused on sales numbers but on deploying theories on motivation, engagement and behaviour changes for improvement in education and healthcare. In business context, various authors provide practical guidelines and successful applications are abundant. These guidelines often list game elements and mechanisms, such as giving rewards and feedback, and give examples of their use in practice. At the same time these methods risk reducing a game or 'gamified' application to a simplified collection of building blocks, which makes it easy to lose sight of the original experience. Advancements in academia move in an entirely different direction. Only several solid frameworks were found, mainly focused on facilitating intrinsic motivation through applying the right game elements and their underlying psychological or behavioural aspects. These frameworks, however, are in their infancy compared to those from business: they are complex, lack empirical evidence and guidelines for use in practice. A major downside of this situation is that new academic initiatives are inclined to make use of the success and practical advantages of business gamification while these methods, however appropriate for sales, lack sufficient profundity and foundation for more complex goals such as in eHealth. The study resulted in a small source of information on how to realise suitable design for the older adult. A few authors identify a substantial challenge in the unfamiliarity of the older adult with (modern) digital games, as their current gaming preferences may not be a proper source of information for new developments.

Furthermore, existing user classifications that may support the development of attractive game design for the older target group for eHealth applications were explored. Such classifications divide players according to an archetype, basing classes on singular types and its associated preferences, or by the attributes that describe the preferences of users, such as motivation or aspects that are found enjoyable. These classifications can be used to provide insight in the game preferences of individuals or (sub)groups, enabling the tailoring of design to these particular (group of) users. Clear parallels were identified upon comparing the classifications along their classes. However, none of these appeared directly applicable to the older audience.

In a series of follow-up studies, covered by chapters 3, 4 and 5, a new framework was developed aimed at facilitating effective application of game-based design in terms of engagement for various target groups. Based on the results of the study described above this new framework should be able to provide a detailed insight in the preferences of older adults, should be scientifically substantiated and relying on proven engagement and motivation strategies. A new user classification was drafted based on one of the classifications from the abovementioned study: the 
'five domains of play' theory. This theory hypothesises a strong correspondence between personality, and the way it can be described and categorised in five factors (according to the 'five factor model'), and motivations in playing games. The most important advantage of this model is that it consists of a number of linear factors instead of relying on stereotypical characterisations, which is the case in player type models. Each of these factors can be rated along multiple underlying facets. In the five domains theory, these factors are translated into 'domains', while perhaps 'dimensions' would be a more accurate term. Altogether these scores form a 'user profile' for either individuals or a group of users.

A pilot study was performed to examine the theory behind this classification and explore the measuring instrument (chapter 3). This study examined the relation between personality and game preferences for different age groups. Participants completed an online questionnaire on personality and game preferences. The correlations between personality and game preferences varied between domains as well as demographic groups. Significant correlations were found for the younger but not for the older population.

A field study was performed subsequently (chapter 4), in which the applicability of the five domains of play theory was further evaluated. The goal was to reexamine the relation between game preferences and personality for older adults in an improved study setting, and to gain more insight in the interpretation of assessed preferences towards tangible game design. It was hypothesised, as mentioned before in chapter 2, that the absence of plausible correlations for the older adult in the previous study was due to their lesser experience with modern video games. Prior to the measurements, participants were therefore asked to play five preselected tablet games as much as they liked during one week. Again, no correlations were found between personality and game preferences for this target group. This relation was therefore left out of further developments of the framework. The study furthermore resulted in a comprehensible overview of the participants' game preferences. Additional to the user profiles, interviews provided a detailed background to the user experience of different game elements. Making modern games accessible for the participants enabled them to more accurately express their preference than they would when based on their prior experience alone. Also, the framework enabled retrospective visualisation and explaining the approval or rejection of the different games by these users.

The last two studies of these chapters (4 and 5) are aimed at empirical evaluation, validation and applicability of the framework for different target groups. The application of the framework to assess (young) children's game preferences was explored (chapter 4), with a focus on survey usability, classification semantics, visualisation of resulting profiles of preferences and the translation into recommendations for game design. The envisioned use of this knowledge was a game for children with exercise induced asthma. A concise version of the survey, fitting the inner world of children, was used to measure game preferences. The 
scores of the different demographic subgroups were analysed and profiles were visualised. This result indicated general similarities and differences in preferences for game elements in boys and girls. Interpretation of these results lead to a series of recommendations for game-based design, which were used in the development of gameplay of a physical game and game-based application for this target group (not described in this thesis).

A two-part study was then performed to refine and complete the framework as a practical method for assessing game preferences in any target audience towards creating engaging game-based design (hoofdstuk 5). The first section of the study consisted of several iterations of the framework and its validation by means of expert sessions, the second part was a field study to demonstrate the application of the framework in practice. In the first section the semantics of the classification and the description of the domains were completed, the survey was optimised in terms of workload for the participant and ease of processing and interpretation of the measured scores, and the content validity was checked. The expert sessions resulted in the final version of the framework: the ' $6 \mathrm{D}$ framework'. The second section covered an online study in a general audience - adults interested in video games to demonstrate the application of the framework to gain insight in the target group's game preferences. This study furthermore investigated the use of the framework to recommend existing games to users based on a match between user preference profiles and game content profiles. The study demonstrated the assessment of game preferences, visualisation and analysis of results, and subdivision of identified user groups. Promising similarities between personal and game profiles were found for a large number of participants, while correlations per domain between the two profiles were not generally convincing. The study concludes that the framework can provide a useful and clear insight in the intended target group, and that first efforts in recommending existing games to users was successful but susceptible to several study limitations. Follow-up research should focus on further validation of the framework and evaluation of the effectiveness of game design based on the preferences assessed by means of the framework.

The effect of tailoring game-based design to the older user on their engagement to eHealth was further examined through two studies (chapter 6 and 7). The first study describes the development of a mobile coaching application that stimulates the older adult in maintaining healthy levels of physical activity. The experience of the user was evaluated in terms of engagement with the application, motivation to be physically active, and how these aspects related to the deployed game design features. The concept for game design in this application was based on the results of an earlier performed study towards the use of digital and conventional games by older adults, and developed further using the study results from the experiments described in this thesis. The game-based application that was developed for this study, WordFit, combined the concept of an existing activity coach and step counter. Daily measured activity and step targets reached controlled the gameplay 
in this application. The game-based application as well as the standard application were offered to the participant for four weeks. Interviews were held to evaluate the overall use experience and appreciation of specific game content, in addition to the measurements of engagement to the application and motivation to be active. Results showed that WordFit did not influence the maintenance of a daily step goal as expected. The newly gained insight of users in their daily activity appeared to be the most important motivation for healthy behaviour as well as for the use of the applications. Some considered the game a valuable addition in drawing attention to the use of the activity coach and monitor, but was mostly valued for being a brain trainer. The game was insufficiently engaging for the target group on several accounts: the concept was not a proper translation of the favourite (board) games older adults previously mentioned, the integration of physical activity in the game was not sufficiently intuitive and the content was insufficiently complex and challenging for long-term use. The framework however enabled analysis of the appreciation for the applied game elements, leading to more detailed knowledge of this target group's preferences. Furthermore, results once more indicated that assessing game preferences of older adults based on their prior experience with games does not sufficiently identify the game elements that can lead to more engagement when translated into game-based design.

A concluding, large scale study encompassed the entire development trajectory of a game-based eHealth application in practice by means of a case (chapter 7). The eHealth application in this case was a self-management platform for older adults providing training on three aspects of a healthy lifestyle: physical and cognitive health and healthy nutrition. Training on these aspects counters and prevents frailty. The development trajectory consisted of four phases: end-user research, conceptualisation, creative design and refinement. The first phase focused on assessing the game preferences of the older adult, thereby reverting to results of prior and new experiments. This lead to preference profiles, now including the perception as well as the appreciation of the user on the same game content in order to better facilitate interpretation of the results by the designer. Concrete recommendations for design for this target group and use context followed. A strategy was then determined to realise long-term engagement. Stakeholders of different fields of expertise of the basic eHealth application (cognitive and physical health and nutrition) were involved in order to translate the performance of the enduser into meaningful gameplay. This information formed the basis for the creative design phase. In this iterative process the central game concept was developed, the progression and translation of actions on the underlying training modules were elaborated, the gameplay for the different areas end levels of the game were designed as well as the concept art for the final graphic appearance. In the final phase, an iterative prototyping and evaluation process resulted in the finished game-based eHealth application. Four versions were evaluated by end-users during three rounds and tested on appreciation, ease of use, expected effectivity and motivation for the underlying training structures, fun and pleasantness in using 
the application and expected future use. The feedback on the final version of the prototype regarding these aspects was positive. For (retrospective) analysis and future developments, more data on the user's game preferences was gathered using the $6 D$ framework.

These two studies provided insight in the development of game-based eHealth in practice. By creating transparency in this process and highlighting the rationale behind design choices and motivation strategies in game-based design, future and existing game-based applications can be optimised. By offering repeatable, practical methods, suitable content, principles and mechanisms can be selected and applied. This thereby defeats the need for uncritically copying from existing applications without a guarantee for success. The case study (chapter 7) resulted in a fully developed, well-functioning and aesthetically attractive game-based eHealth application. Also, evaluations with end-users showed that the preferences as investigated with the 6D framework, and resulting strategies for long term engagement, have led to game content that engages the older adult user. Suggestions for future research are towards generalising results towards a broader, elderly audience, as the participants of the studies in this thesis were inherently interested in technological advancements, research or games, as well as the roll-out of developed applications in practice.

\section{Samenvatting}

Digitale zorgtoepassingen, ook wel 'eHealth' genoemd, kunnen bijdragen aan het verhogen van de kwaliteit van de gezondheidszorg en het verlichten van de zorgvraag. Zo kan bijvoorbeeld op afstand worden gemonitord en behandeld, en kan de patiënt meer grip op de eigen gezondheid krijgen door de inzet van zelfmanagement applicaties en door betere toegankelijkheid van diensten. Een snel groter wordende doelgroep die veel profijt kan hebben van eHealth is de oudere populatie (65 jaar en ouder). Voor deze groep kan eHealth onder meer bijdragen aan het verlengen van de zelfredzaamheid en zelfstandigheid door te ondersteunen in het aannemen of onderhouden van een gezonde(re) leefstij|. Het is van groot belang dat eHealth applicaties worden gebruikt zoals ze bedoeld zijn: veelal frequent en langdurig. Vaak laat deze zogenaamde 'therapietrouw' nog te wensen over, waardoor er niet optimaal geprofiteerd kan worden van de geboden interventie. Spellen ('games'), of specifieke spelelementen, kunnen vanwege hun natuurlijke aantrekkingskracht op mensen worden ingezet in toepassingen die vermaak niet als primair doel hebben. Door een boodschap of doel in een computerspel te verpakken ('serious games') of concrete onderdelen van spellen aan bestaande applicaties toe te voegen ('gamification') tracht men het gebruik van deze toepassingen aan te moedigen. Ook binnen de gezondheidszorg is deze aanpak, die we in dit proefschrift met een overkoepelende term 'game- 
based design' noemen, niet onopgemerkt gebleven. Bij doelstellingen waarvoor niet zelden saaie, herhalende en belastende taken moeten worden uitgevoerd valt nameliik veel winst te halen met een effectieve motivatiestrategie.

Dit proefschrift beschrijft hoe de kracht van game-based design om mensen te boeien en hun aandacht vast te houden - wat we met de Engelse term 'engagement' aanduiden - voor productieve doeleinden ingezet kan worden, om daarmee in te spelen op de vraag naar strategieën om het gebruik van eHealth applicaties te verbeteren. Hierbij ligt specifiek, maar niet vitsluitend, de focus op het aanspreken van de oudere populatie. Oftewel, dit proefschrift beschriift hoe game-based eHealth applicaties moeten worden ontworpen om meer 'engagement' te creëren bij de oudere gebruiker. Hoofdstuk 1 zet de achtergrond van dit onderzoek, zowel voor eHealth als game-based design, in meer detail viteen.

De eerste studie van dit proefschrift, beschreven in hoofdstuk 2, werd gedaan naar de huidige stand van zaken omtrent het concept gamification. Directe aanleiding voor deze studie was het zien ontstaan van vele initiatieven vanuit diverse achtergronden en onderzoeksgebieden die van gamification gebruikmaakten, maar het ontbreken van een eenduidige conceptualisatie van het begrip. Welke aanpak voor gamification het meest effectief lange termijn engagement zou kunnen realiseren bleek, met name voor de oudere gebruiker, een nog weinig onderzocht onderwerp: methoden om game-based design toe te passen, voorzien van zowel een solide theoretische onderbouwing als praktische richtlijnen, zijn niet ruim voorhanden. Deze studie heeft geleid tot een overzicht van gamification activiteiten zowel binnen als buiten wetenschappelijk onderzoek, zoals de gaming industrie en het bedriffsleven. Het doel hiervan was te voorzien in een begrip van de theoretische achtergrond van gamification en inzicht te geven in bestaande conceptuele modellen ('frameworks') om gamification te ontwikkelen, zowel voor algemeen als voor ouder publiek. Daarbii zijn bestaande gebruikersclassificaties die zouden kunnen worden gebruikt om doelgericht spelelementen te kunnen ontwerpen voor specifieke gebruikers verkend.

Uit de resultaten kwam naar voren dat de conceptualisatie van gamification en definities van bijbehorende begrippen, zoals de onderdelen van spellen oftewel game elements, op twee manieren wordt beschouwd. Enerzijds is er gamification zoals toegepast in zakelijke, marketing en klantgerichte context; anderzijds is er gamification die niet is gericht op verkoopcijfers maar juist op het toepassen van theorieën over motivatie, engagement en gedragsverandering voor doelen zoals betere educatie en gezondheidsuitkomsten. In zakelijke context zien we talriike auteurs die zeer concrete richtlijnen voor het toepassen van gamification bieden, alsmede succesvolle toepassingen in de praktijk. Deze richtlijnen bestaan uit opsommingen van spelelementen en -mechanismen, zoals het geven van beloningen en het geven van feedback, en geven praktische voorbeelden voor de toepassing. Tegelijkertijd reduceren deze methoden een spel, of een 'gamified' applicatie, vaak 
tot een eenvoudige verzameling bouwstenen waardoor de diepgang en originele ervaring van spellen makkelijk uit het oog kan worden verloren. Bijna haaks hierop staan de ontwikkelingen binnen de academische wereld. Enkele solide frameworks werden gevonden, vooral gericht op het faciliteren van de intrinsieke motivatie door het toepassen van de juiste spelelementen en onderliggende psychologische of gedragswetenschappelijke factoren. Deze frameworks staan echter in vergelijking nog maar in de kinderschoenen: ze zijn complex en het ontbreekt aan empirisch bewijs en handvatten voor toepassing in de praktijk. Het grote nadeel van deze situatie is dat nieuwe initiatieven in academia dankbaar gebruikmaken van het succes en de praktische voordelen van gamification voor zakelijke doeleinden, terwijl deze methoden, hoewel toereikend voor bijvoorbeeld marketing, in veel gevallen te weinig diepgang bieden voor het behalen van de complexe doelen zoals die bijvoorbeeld in eHealth aan de orde zijn. Tevens resulteerde deze eerste studie in een slechts kleine bron van informatie over hoe geschikt ontwerp voor de oudere gebruiker kan worden gerealiseerd. Enkele auteurs stellen dat een grote vitdaging ligt in de onbekendheid van veel ouderen met (moderne) digitale spellen, waardoor huidige spelvoorkeuren geen goede informatiebron zijn om nieuwe spelcontent op te baseren.

De studie onderzocht ook bestaande gebruikersclassificaties die ondersteuning zouden kunnen bieden in het creëren van aantrekkelijk game design binnen de ontwikkeling van eHealth applicaties. Dergelijke classificaties delen spelers in op basis van een archetype waarbij klassen gebaseerd zijn op singulaire types met daarmee geassocieerde voorkeuren, zoals 'player type' of persoonlijkheid, of op basis van de attributen die de voorkeuren van gebruikers omschrijven, zoals motivatie of wat men precies als plezierig ervaart. Gebruik van dergeliike classificaties kan inzicht geven in de voorkeuren ten opzichte van spelelementen van verschillende individuen of (sub)groepen, wat mogelijk maakt het ontwerp toe te spitsen op deze (groep van) gebruikers. Bij het vergelijken van deze verschillende classificaties middels hun klassen werden duidelijke parallellen gevonden. Echter werd geen van de classificaties geschikt bevonden voor een ouder publiek.

In een reeks vervolgstudies, beschreven in de hoofdstukken 3, 4 en 5, is een nieuw framework ontwikkeld om het effectief toepassen van game-based design in applicaties voor verschillende doelgroepen te faciliteren. Op basis van bovenstaande resultaten werd gesteld dat dit nieuwe framework gedetailleerd inzicht in de voorkeuren van oudere spelers moet kunnen bieden, voorzien van grondige wetenschappelijke onderbouwing en gebruikmakend van bewezen strategieën op gebied van motivatie en engagement. Een nieuwe gebruikersclassificatie werd opgesteld met als uitgangspunt een van de classificaties uit bovengenoemde studie (hoofdstuk 2), namelijk de 'five domains of play' theorie. Deze theorie stelt dat motivatie om te spelen sterke overeenkomsten vertoont met persoonliikheid, en de manier waarop we deze beschrijven en indelen aan de hand van vijf categorieën 
(aldus het 'five factor model'). Een belangriik voordeel van dit model ten opzichte van bijvoorbeeld spelerstypologieën die gebaseerd zijn op het toewijzen van één bepaald (stereo)type aan een persoon, is dat het is opgebouwd uit een reeks lineaire factoren. Op elk van deze factoren, kan middels een reeks onderliggende facetten gescoord worden. Deze factoren worden, vertaald naar motivatie om te spelen, 'domains' of domeinen genoemd, hoewel 'dimensies' een meer toepasselijke term zou zijn. Hiermee kan een 'profiel' van de gebruiker, of een groep gebruikers, worden gemaakt.

Om de theorie achter deze classificatie te toetsen en een meetmethode te verkennen werd een pilot studie uitgevoerd (hoofdstuk 3). In deze studie werd onderzocht of een verband tussen persoonlijkheid en spelvoorkeuren ook daadwerkelijk kon worden aangetoond voor verschillende leeftijdsgroepen. Deelnemers vulden online een vragenlijst in waarmee een beeld werd gevormd van zowel de spelvoorkeuren als de persoonlijkheid van de deelnemer. De correlaties tussen persoonlijkheid en spelvoorkeuren bleek zowel te variëren tussen domeinen als tussen demografische groepen. Significante correlaties werden in deze studiesetting wel voor jongeren gevonden maar niet voor ouderen.

Vervolgens werd een veldstudie gedaan (hoofdstuk 4) waarin de toepasbaarheid van de five domains of play theorie verder werd verkend voor deze doelgroep. Het doel was om de relatie tussen spelvoorkeuren en persoonlijkheid voor ouderen opnieuw te toetsen in een verbeterde studiesetting en om meer inzicht te krijgen in de interpretatie van vastgelegde voorkeuren om te komen tot concreet spelontwerp. De hypothese was dat afwezigheid van aannemelijke correlaties voor de oudere gebruiker in de voorgaande studie veroorzaakt werd door de beperktere ervaring van de oudere gebruiker met moderne computerspellen, zoals eerder genoemd in hoofdstuk 2. Voorafgaand aan de metingen werd deelnemers gevraagd een vifftal hiervoor geselecteerde games op een tablet naar wens te spelen gedurende een week. Ook in deze studiesetting was de correlatie tussen spelvoorkeuren en persoonlijkheid voor de oudere doelgroep niet evident. Het verband met persoonlijkheid is derhalve losgelaten in de verdere ontwikkeling van het framework. Wel resulteerde de studie in een helder en inzichtelijk beeld van de spelvoorkeuren van de gebruikers. Interviewresultaten gaven een gedetailleerd beeld van de beleving van de verschillende aspecten van de games, in aanvulling op deze profielen van spelvoorkeur. Door de gebruiker toegang te geven tot moderne computerspellen werd een achtergrond aangereikt dat hen in staat stelde hun voorkeuren nauwkeuriger te motiveren dan slechts op voorgaande ervaring met spellen. Tevens kon retrospectief de voorkeur of afkeur voor de verschillende games door middel van het nieuwe framework eenvoudig visueel inzichtelijk gemaakt en verklaard worden.

De laatste twee studies van deze hoofdstukken (4 en 5) zijn gericht op empirische evaluatie, validatie en inzetbaarheid van het framework voor verschillende 
doelgroepen. De toepassing van het framework om de spelvoorkeuren van (jonge) kinderen uiteen te zetten werd verkend (hoofdstuk 4), met focus op toegankelijkheid van de vragenlijst, de semantiek van de classificatie, de visualisatie van de resulterende profielen van spelvoorkeuren en de vertaling daarvan naar aanbevelingen voor game design. De beoogde toepassing van deze kennis was een game voor kinderen met inspanningsastma. Een beknopte versie van de vragenlijst, passend bij de belevingswereld van kinderen, werd afgenomen om spelvoorkeuren te meten. Vervolgens werden de scores van verschillende demografische groepen geanalyseerd en de profielen gevisualiseerd. Dit resultaat toonde globale overeenkomsten en verschillen opgemaakt tussen de voorkeuren voor spelelementen van jongens en meisjes. Interpretatie van deze resultaten resulteerde in een reeks aanbevelingen voor game-based design, welke werden gebruikt bij het ontwerpen van de gameplay (interactie en spelregels, letterlijk speelwijze) van een fysieke game en game-based applicatie voor deze doelgroep (hetgeen niet is beschreven in dit proefschrift).

Het framework werd verfijind en gecompleteerd als praktische methode om de spelvoorkeuren in een beoogde doelgroep te verkennen om engaging game-based design te kunnen creëren in een tweedelige studie (hoofdstuk 5). Het eerste deel van de studie besloeg enkele iteraties van het framework en validatie middels expert sessies, het tweede deel een praktijkstudie om de toepassing van het framework te demonstreren. In het eerste deel werd de semantiek van de classificatie en de omschrijvingen van de domeinen voltooid, werd de vragenlijst gereduceerd tot een optimale omvang in verhouding tot de belasting voor de eindgebruiker en het gemak de gemeten scores te verwerken en interpreteren, en werd de 'content validity' (inhoudsvaliditeit) gecontroleerd. De expert sessies leverden de viteindelijke versie van het framework op: het '6D framework'. In het tweede deel werd middels een online studie in een algemeen publiek - volwassenen met een interesse in computerspellen - de toepassing van het 6D framework gedemonstreerd om een impressie van de voorkeuren van dit publiek te verkriigen. Tevens werd in dit deel van de studie onderzocht of het framework zou kunnen worden gebruikt om bestaande spellen te kunnen aanbevelen op basis van de match tussen profielen van de voorkeuren van gebruikers en van die van de aanwezige game content in bestaande video games. De studie demonstreerde hoe spelvoorkeuren werden gemeten, gevisualiseerd en geanalyseerd, en onderverdeeld in significant verschillende subgroepen. Een veelbelovende overeenkomst tussen persoonlijke en game profielen werd gevonden voor een groot aantal deelnemers, echter waren correlaties per domein tussen de twee profielen niet algemeen overtuigend. De studie concludeert dat het framework een nuttig en helder inzicht in de beoogde doelgroep kan verschaffen, en dat een eerste aanzet naar het doen van aanbevelingen met bestaande games succesvol was maar onderhevig aan een aantal beperkingen vanuit de studie. Toekomstig onderzoek zou zich moeten richten op verdere validatie van het framework en evaluatie van de effectiviteit van game design gebaseerd op de met het framework in kaart gebrachte voorkeuren. 
Het effectvan het toespitsen van game-based design op de engagement bii de oudere doelgroep in eHealth werd middels twee studies verder onderzocht (hoofdstuk 6 en 7). De eerste studie beschrijft de ontwikkeling van een mobiele coaching applicatie die ouderen stimuleert in het onderhouden van dagelijkse fysieke activiteit. Daarbii werd de ervaring van de gebruiker geëvalueerd op gebied van engagement met de applicatie, motivatie om fysiek actief te zijn, en hoe deze aspecten zich tot het toegepaste game design verhouden. Het concept voor het game design werd op de vitkomsten van een al eerder vitgevoerd onderzoek naar het gebruik van digitale en conventionele spellen door ouderen gebaseerd, en vervolgens verder vitgewerkt aan de hand van studieresultaten van het onderzoek beschreven in dit proefschrift. De game-based applicatie die ontwikkeld werd voor deze studie, WordFit, combineerde het concept van een bestaande activiteitencoach met een stappenteller. De gameplay werd in deze applicatie aangestuurd door de dagelijks gemeten activiteit en daarmee behaalde doelstellingen. De game-based applicatie en de standaard applicatie werden gedurende vier weken aangeboden. In aanvulling op de gemeten engagement met de applicatie en motivatie om actief te zijn werden na afloop interviews afgenomen om het algemeen gebruik en waardering voor specifieke game content te evalueren. Resultaten wezen uit dat WordFit niet op de verwachte manier invloed had op het aanhouden van actief gedrag. Het nieuw verkregen inzicht van gebruikers in hun dagelijkse activiteit bleek de grootste drijfveer voor zowel gebruik van de applicaties als fysieke activiteit. De game werd soms als een waardevolle aanvulling gezien om de aandacht terug te brengen naar de activiteitencoach en -monitor, maar werd met name gewaardeerd als breintrainer. De game was op een aantal vlakken onvoldoende engaging voor de doelgroep: het concept bleek niet de juiste vertaling te zijn van de door ouderen genoemde favoriete (bord)spellen, de integratie van fysieke activiteit in het spel bleek niet intuïtief genoeg en de content was ontoereikend in complexiteit en vitdaging voor lange termijn gebruik. Wel konden de toegepaste spelelementen met het framework geanalyseerd worden wat leidde tot meer gedetailleerde kennis over de spelvoorkeuren van deze doelgroep. De resultaten toonden bovendien aan dat eenvoudigweg vragen naar voorkeuren op basis van voorgaande ervaring onvoldoende identificeert welke spelelementen in een vertaalslag naar game-based design voor deze doelgroep daadwerkelijk tot meer engagement leiden.

Een laatste, grootschalige studie omvatte de gehele ontwikkeling van een gamebased eHealth applicatie in de praktijk aan de hand van een case (hoofdstuk 7). De eHealth applicatie in deze case was een self-management plafform voor de oudere gebruiker waarin kan worden getraind op drie factoren van een gezonde leefstijl: fysieke en cognitieve fitheid en gezonde voeding. Door deze factoren op peil te houden kan kwetsbaarheid bij ouderen worden verminderd. In het ontwikkeltraject werden vier fasen onderscheiden: vooronderzoek naar de eindgebruiker, conceptualisatie, creative design en verfijning. In de eerste fase 
werden spelvoorkeuren voor ouderen onderzocht, puttend vit zowel de eerder beschreven studies als voor deze studie nieuwe experimenten. Dit leidde tot voorkeursprofielen, waarin ditmaal zowel de perceptie als de waardering van de gebruiker op dezelfde spelcontent werd vastgelegd om de interpretatie door de ontwerper te vergemakkelijken. Hieruit volgden concrete ontwerpaanbevelingen voor deze doelgroep en gebruikscontext. Vervolgens werd een strategie bepaald om lange termijn engagement te realiseren. Stakeholders van de verschillende expertises van de basis eHealth applicatie lop gebied van fysieke en cognitieve gezondheid en voedingl werden hierin betrokken om prestaties van de eindgebruiker op een zinvolle manier te kunnen verwerken in de gameplay. Deze informatie vormde de basis voor de creative design fase. In dit iteratieve proces werd het centrale spelconcept bedacht, de progressie en de vertaling van acties op de onderliggende trainingen in de game vitgewerkt, de gameplay voor de verschillende locaties en niveaus in de game en het grafisch concept ontworpen. In de laatste fase resulteerde een iteratief prototyping- en evaluatieproces in de viteindelijke game-based eHealth applicatie. Vier versies werden in drie rondes geëvalueerd door eindgebruikers en getoetst op waardering, gebruiksgemak, verwachte effectiviteit en motivatie voor de onderliggende trainingsstructuren, plezier en voldoening in het gebruiken van de applicatie en verwacht toekomstig gebruik. De terugkoppeling op de viteindelijke versie van het prototype aan de hand van deze aspecten was positief. Ook werd gedurende deze fase informatie verzameld over de spelvoorkeuren van de gebruiker met het 6D framework voor (retrospectieve) analyse en toekomstige ontwikkelingen.

Deze twee studies verschaften inzicht in het ontwikkelingsproces van game-based eHealth in de praktijk. Door dit proces inzichtelijk te maken en aandacht te geven aan de rationale achter ontwerpkeuzes en motivatiestrategieën in game-based design kan het ontwerp van bestaande en nieuwe game-based applicaties worden geoptimaliseerd. Door het aanbieden van herhaalbare, praktische methoden kan geschikte content, principes en mechanismes worden geselecteerd en toegepast, en hoeft men niet meer klakkeloos van bestaande applicaties te kopiëren zonder garantie op succes. De case studie (hoofdstuk 7) resulteerde in een volledige, goedwerkende en aantrekkelijk ogende game-based eHealth applicatie. Ook toonden evaluaties met eindgebruikers aan dat de resultaten van het voorgestelde gebruikersonderzoek, met behulp van het 6D framework en daaruit voortkomende strategieën voor lange termijn engagement, hebben geleid tot spelcontent die engaging is voor de oudere gebruiker. Suggesties voor vervolgonderzoek zijn het generaliseren van resultaten naar het brede oudere publiek, daar de eindgebruikers in de studies beschreven in dit proefschrift uit zichzelf interesse toonden in technologische ontwikkelingen en games, en het vitrollen van de toepassingen in de praktijk. 


\section{Dankwoord}

Voor u ligt mijn proefschrift: het resultaat van vijf jaar werk. Vier van deze jaren werkte ik met veel plezier aan Universiteit Twente, één jaar met iets minder plezier vanuit onze woonkamer. Daarom ben ik niet minder trots op dit resultaat. Misschien was het wel genoeg geweest na die vier jaar, maar ik zou mezelf niet zijn als ik er niet het allerbeste vit had willen halen. Dit alles had ik niet kunnen doen zonder de bijdrage van vele anderen. $U$, die dit boekje in handen heeft, bent waarschijnlijk een van deze personen. Er is echter een aantal personen dat ik in het bijzonder wil bedanken. Zonder hen was dit proefschrift niet geworden wat het nu is.

Ten eerste gaat mijn dank uit naar de vele deelnemers van de studies die zijn opgenomen in dit proefschrift. Zij hebben met veel enthousiasme applicaties getest, spelletjes gespeeld, soms saaie vragenlijsten ingevuld en mij hartelijk ontvangen als er interviews moesten worden afgenomen. Niet alleen maakte dit de vitvoering van deze studies voor mij ontzettend leuk, in alle gevallen hebben zij ook een zeer waardevolle bijdrage geleverd waarvan veel in dit proefschrift kan worden teruggevonden. Onder deelnemers reken ik niet alleen de eindgebruiker van de studies maar ook alle anderen die deelname voor hen direct of indirect mogelijk maakten: fysiotherapeuten en vakspecialisten, zorginstellingen, mantelzorgers, ouders en andere betrokkenen.

Dan wil ik mijn promotor, Miriam Vollenbroek, co-promotor Hermie Hermens en mijn dagelijks begeleider Monique Tabak bedanken. Jullie begeleiding en visie waren essentieel voor wat dit proefschrift is geworden. Tegelijkertijd heb ik de vrijheid gehad veel van mijn studies zelf op te zetten en in te richten. Miriam, ik wil je bedanken voor de energie die je van begin tot eind in mijn promotietraject hebt gestoken. Bedankt voor je enthousiasme voor mijn werk en voor de inspiratie voor de grote onderzoekslijn, maar ook voor mij als persoon. Zelfs op momenten dat ik er zelf het vertrouwen in begon te verliezen wist je me te motiveren met een positief inzicht. Ik ben er trots op dat ik bij jou mag promoveren. Hermie, we hebben meer gepraat over science fiction, reizen, koken en allerlei andere interessante zaken dan over mijn artikelen. Dat is niet omdat we het zo weinig over mijn onderzoek hebben gehad, in tegendeel zelfs. Ook onze discussies over het leukste deel van het onderzoek, games natuurlijk, hebben me erg geholpen. Bedankt voor je aandacht en voor je humor. Monique, dank je voor je betrokkenheid. Ik heb veel van je geleerd. In onze vele discussies wist je altijd precies de juiste vragen te stellen, wat bij mij niet zelden lichte paniek veroorzaakte. Ook je grondige en kritische blik op mijn artikelen zijn de kwaliteit ervan zeer ten goede gekomen. Bedankt dat ik je eerste promovendus mocht zijn.

Als ik me niet zo thuis had gevoeld op de UT was het niets geworden met dit proefschrift. De staf en al mijn collega's van de vakgroep BSS wil ik daarom 
hartelijk danken voor de fijne werksfeer en de dagelijkse gezelligheid. Velen van jullie zijn mijn vrienden geworden. Wies, vooral door jou voelde ik me vanaf het begin welkom. In het bijzonder wil ik de mensen bedanken die me het meest van mijn werk hebben gehouden met reizen, whisky tastings, fietstochties, borrels, barbecues, festivals en andere feestijes: Frauke, Kees, Henk, Dirk, Fokke en RobertJan. Ook mijn kamergenoot Hossein mag niet aan dit rijtie ontbreken. Ik wil je niet alleen bedanken voor de gezelligheid op ons kantoor en de leuke samenwerking die we hadden in projecten, maar ook voor alle keren dat we bij jou en Milad thuis welkom waren. Ainara and Fernando, you are truly amazing. Thank you for your warmth and inspiration.

Wendy, jii hoort thuis in meer dan één categorie van mensen om te bedanken. Een betere kamergenoot heb ik me niet kunnen wensen: het was altijd leuk met jou. Van kunst uitzoeken tot haring halen, uitjes organiseren en zeilen. Ook wil ik je bedanken voor je nauwe betrokkenheid bij de laatste fase van mijn onderzoek. Zelfs in jouw overvolle agenda vond je nog ruimte om mijn werk te lezen en van commentaar te voorzien. Zonder jouw hulp was hoofdstuk 7 er zeker niet gekomen. Dankje voor je vriendschap en het tolereren van 'hysterische jazz'. Ik vind het een eer dat je mijn paranimf wilt zijn.

Dank aan mijn collega's van het RRD met wie ik samen heb gewerkt in diverse projecten. Niet uitsluitend maar wel in het bijzonder Miriam, Stephanie, Marit en Lex. Het was niet alleen een prettige en inspirerende samenwerking, het was vaak ook erg gezellig tijdens bijeenkomsten en vergaderingen in het buitenland. Bedankt voor de fantastische inside tour in jouw stad Lissabon, Miriam, en dat jullie me naar huis hebben gesleept toen ik halfdood van een voedselinfectie nog wat langer in Napels moest blijven dan me lief was. Marit, jou wil ik nogmaals bedanken voor de keren dat je het tijdelijk van Monique over hebt genomen en me vooruit hebt geholpen wanneer ik vastliep. Bij deze kriig je eindelijk ook het statistiekboek terug waar ik bij elke studie gebruik van heb gemaakt.

Ik prijs me gelukkig met een aantal goede vrienden waar ik altijd op kan rekenen. Zonder allen bij naam te noemen wil ik jullie bedanken voor jullie interesse, vriendschap en gezelligheid, en voor de nodige ontspanning buiten werktijd. Dank dat jullie er waren om bijzondere momenten met ons te delen. Ik hoop dat je er ook vandaag, want wie leest niet het dankwoord voorafgaand aan de ceremonie, bij bent om met me te vieren dat ik nu echt klaar ben met mijn onderzoek. Onder jullie zijn ook mensen die de laatste tijd van mij niet de aandacht hebben gekregen die ze zouden verdienen. Dat jullie me niet uit het oog zijn verloren waardeer ik des te meer, en ik beloof dat ik de schade in zal halen. Een speciaal woord van dank voor Ruben en de leden van Lazz. Jullie zijn in korte tijd een onmisbaar deel van mijn leven gaan uitmaken. Maurice, jou kan ik toch niet onbenoemd laten. Dank voor alle jaren vriendschap, humor, inspiratie en steun. 
Lieve familie. Het lijkt soms zo vanzelfsprekend dat jullie er allemaal zijn. Juist dat vind ik ontzettend bijzonder. Hans en Johanna, papa en mama, jullie maakten mogelijk dat ik zorgeloos kon studeren. In alle jaren daarvoor hebben jullie me van kleins af aan eindeloos van alles vitgelegd en laten zien, boeken aangesleept en museumkaartjes gekocht. Maar ook voor de kern van dit proefschrift zijn jullie aan te wijzen als oorzaak, want van jullie kreeg ik op 9-jarige leeftijd - en dat moet niet zelden zonder spijt achteraf zijn geweest - een Super Nintendo. Nu heb ik het eindelijk bewezen: 'altijd maar die computerspelletjes' waren wel degelijk nuttig! Jasmien, niet alleen hebben we vroeger heel wat uurtjes gespeeld samen, je hebt me ook enorm geholpen tijdens mijn promotie. Bedankt dat je mijn statistiek vraagbaak wilde zijn en gewillig eerste slachtoffer van elke volgende nieuwe applicatie en vragenlijst. Ik ben erg trots dat mijn zusje me ook nu weer bijstaat, als paranimf bij mijn verdediging. Met jou erbij weet ik zeker dat alles goed komt. Rudy en Alberdien, Jan en Annemien, en in gedachten opa en oma, als iemand me vraagt waar ik ben opgegroeid twijfel ik altiid een kort moment. Voor mijn gevoel is dat namelijk evengoed bij jullie in Markelo als thuis in de Achterhoek. Als ik de gelukkigste momenten uit mijn jeugd uit zou moeten kiezen zijn het al die keren dat we met de hele familie bij elkaar kwamen. Om iets te vieren of gewoon zomaar, want een feest was het altijd - meestal tot het moment dat Jan en ik niet meer te hanteren waren. Inmiddels met versterking van Coen, Carlijn en Merel zie ik nog steeds uit naar elke volgende gelegenheid. Dank jullie allemaal voor jullie onvitputtelijke en onvoorwaardelijke aandacht, energie en steun!

Veel dank aan mijn schoonfamilie, Chris, Caroline, Maartie, Jules en Jelte, Nils en Meike, voor jullie betrokkenheid en enthousiasme. Jullie lijken altijd nóg blijer te zijn dan ikzelf bij het bereiken van de volgende miilpaal. Geweldig dat ik ook van jullie familie deel uit mag maken!

Sander, allerliefste, mijn steun en toeverlaat: zonder jou kan ik niet zijn wie ik ben. Dankje dat je me alle vrijheid en ruimte geeft - wat zelfs in letterlijke zin op te vatten is - en me overal vol enthousiasme in bijstaat. Ook in praktisch opzicht heb je het voor mij mogelijk gemaakt om mijn onderzoek af te maken en daar ben ik je zeer dankbaar voor. Jij maakt elke dag mooier en ik kan niet wachten om met jou aan een nieuw hoofdstuk te beginnen.

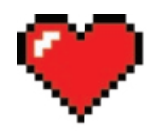





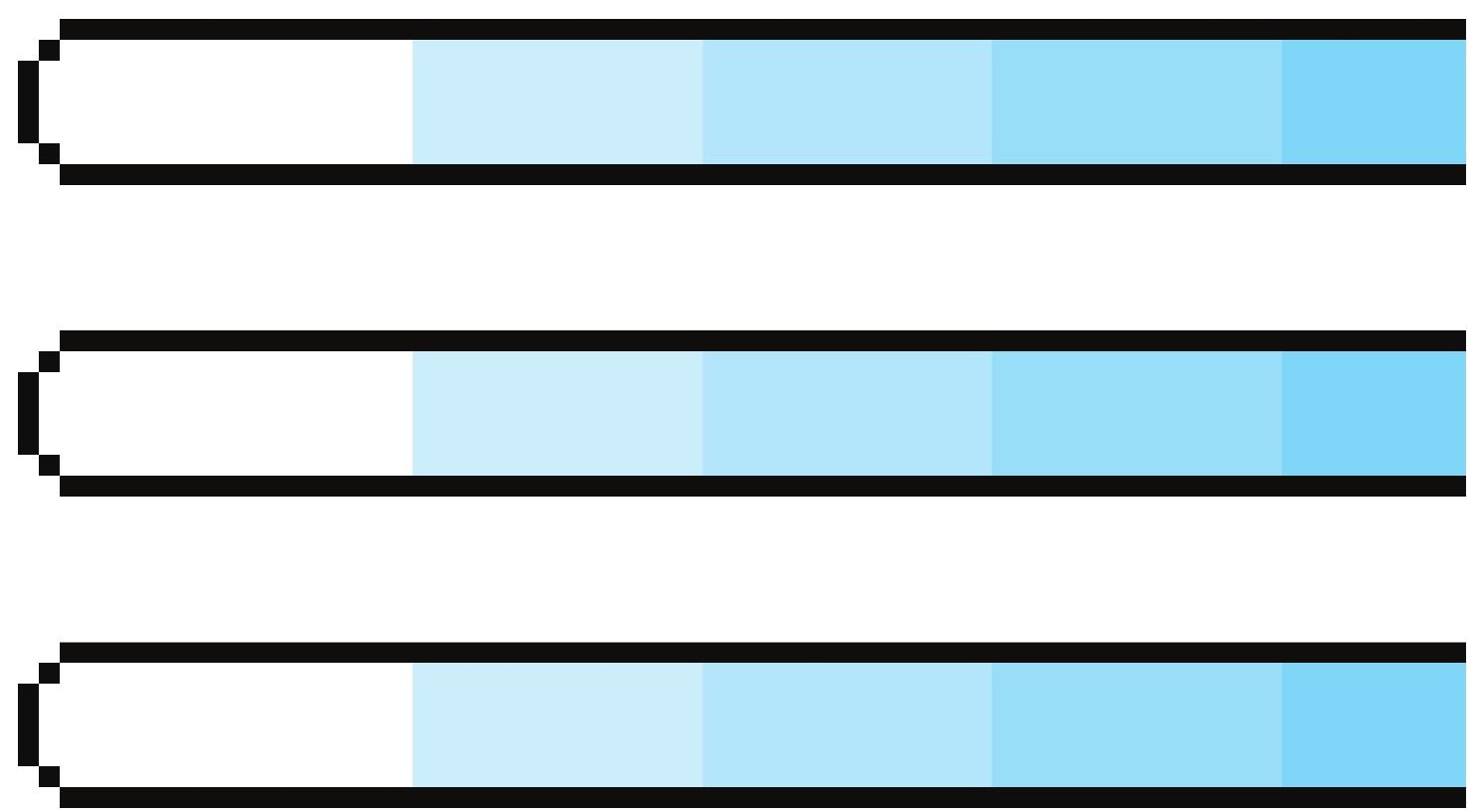

ISBN: 978-90-365-4799-4 